\title{
Menschen im Aufbruch
}

Universitätsbibliothek und Archiv der Universität Wien im Selbstverständnis ihrer Mitarbeiter_innen

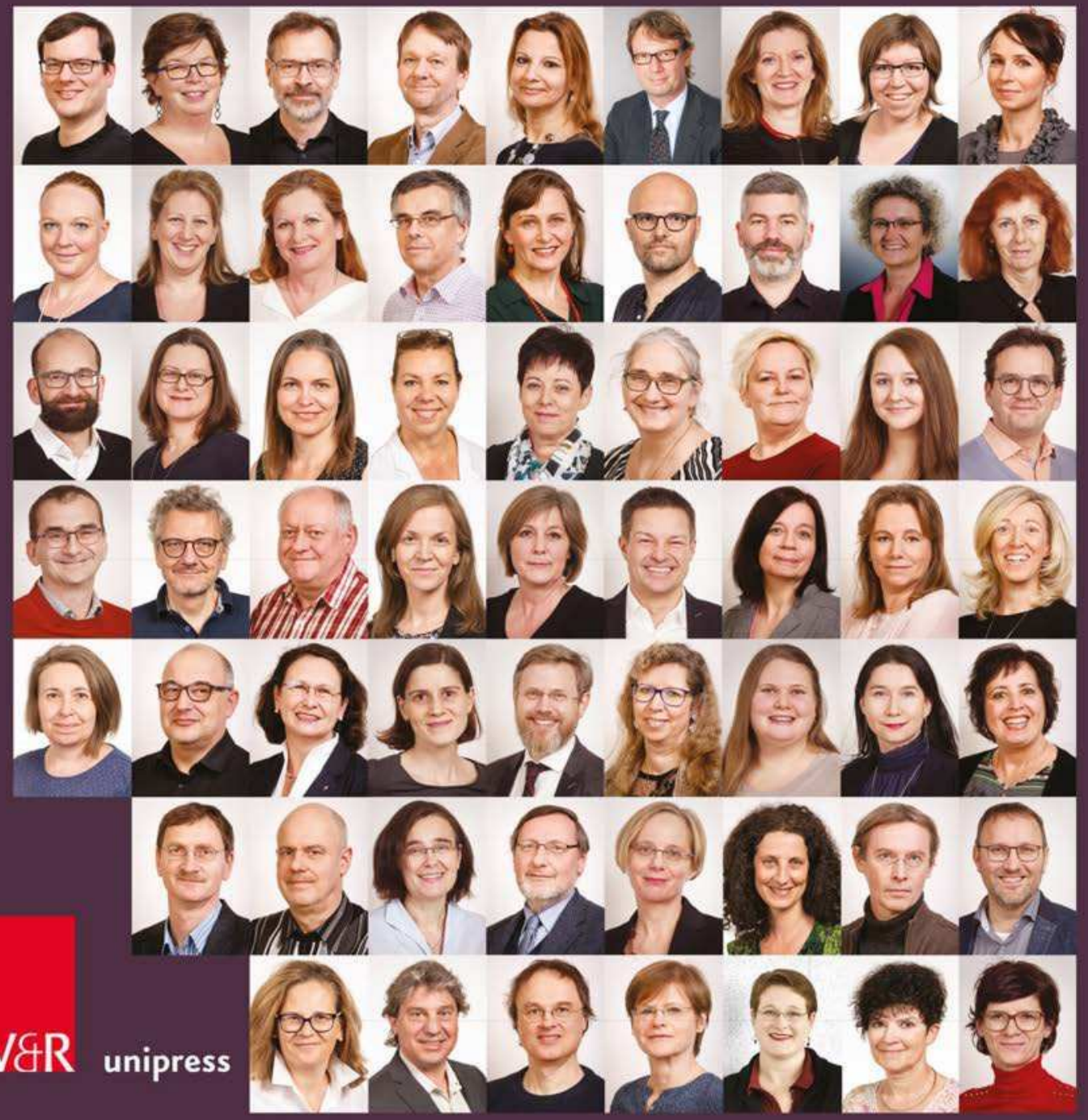


Open-Access-Publikation im Sinne der CC-Lizenz BY-NC-ND 4.0

(c) 2019, Vandenhoeck \& Ruprecht GmbH \& Co. KG, Göttingen ISBN Print: 9783847110989 - ISBN E-Lib: 9783737010986 


\section{Stefan Alker-Windbichler / Claudia Feigl / Christina Köstner-Pemsel / Thomas Maisel / Wolfgang Nikolaus Rappert / Pamela Stückler / Markus Stumpf (Hg.)}

\section{Menschen im Aufbruch}

Universitätsbibliothek und Archiv der Universität Wien im Selbstverständnis ihrer Mitarbeiter_innen

\section{Festschrift für Maria Seissl}

Mit einem Vorwort von Univ.-Prof. Dr. Regina Hitzenberger, Vizerektorin der Universität Wien

Mit 19 Abbildungen

V\&R unipress 
Bibliografische Information der Deutschen Nationalbibliothek

Die Deutsche Nationalbibliothek verzeichnet diese Publikation in der Deutschen

Nationalbibliografie; detaillierte bibliografische Daten sind im Internet über

http://dnb.d-nb.de abrufbar.

(C) 2019, Vandenhoeck \& Ruprecht GmbH \& Co. KG, Theaterstraße 13, D-37073 Göttingen

Dieses Werk ist als Open-Access-Publikation im Sinne der Creative-Commons-Lizenz BY-NC-ND International 4.0 (»Namensnennung - Nicht kommerziell - Keine Bearbeitungen«) unter dem DOI 10.14220/9783737010986 abzurufen. Um eine Kopie dieser Lizenz zu sehen, besuchen Sie https://creativecommons.org/licenses/by-nc-nd/4.0/.

Jede Verwertung in anderen als den durch diese Lizenz zugelassenen Fällen bedarf der vorherigen schriftlichen Einwilligung des Verlages.

Umschlagabbildung: derKnopfdrücker.com (cc-by-4.0), Foto Hausberger: ๑ Michael Bernkopf / Vetmeduni Vienna, Foto Brandtner: (๑) Andreas Brandtner

Vandenhoeck \& Ruprecht Verlage | www.vandenhoeck-ruprecht-verlage.com

ISBN 978-3-7370-1098-6 


\section{Inhalt}

Regina Hitzenberger

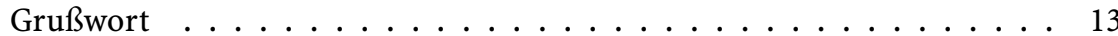

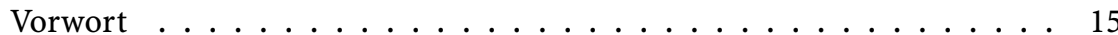

Wolfgang Nikolaus Rappert

Einführung: Die Dienstleistungseinrichtung Bibliotheks- und Archivwesen im Überblick . . . . . . . . . . . . . . . . . . . . . . . . . . . . 17

\section{Zentrale Services und Arbeitsgruppen}

Christa Fried

Wo die Fäden zusammenlaufen. Das Büro Bibliotheks- und Archivwesen als zentrale Clearingstelle für Verwaltung und Finanzen . . . . . . . . 25

Brigitte Höglinger

Vierzig Jahre Personal- und Statistikgeschichte des Bibliotheks- und Archivwesens der Universität Wien. Die Arbeit der Direktionsassistenz . 29

Pamela Stückler

Man kann nicht nicht kommunizieren ... Team Öffentlichkeitsarbeit . . . 33

Alina Rezniczek

Lifelong Learning for Librarians. Das Angebot des Teams Aus- und

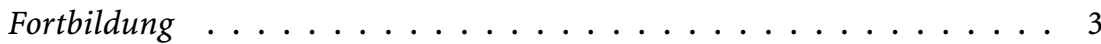

Adelheid Mayer Innovation zwischen Vision und Realität. Die Stabstelle $u b w$ :innovation an der Universitätsbibliothek Wien . . . . . . . . . . . . . . . 43 
Horst Prillinger

Die Webredaktion im Spannungsfeld . . . . . . . . . . . . . 47

Brigitte Kromp / Wolfgang Mayer

The Times They Are A-Changin'. Zentrales Service Clearingstelle

Konsortien, Zentrales Service E-Resource Management ． . . . . . . . 53

Petra Pichler / Christian Beiler

Filialen, Shopping-Welt und Retro-Chic. Der Wandel des Teams

Integrierte Medienbearbeitung Fachbereichsbibliotheken . . . . . . . . . 59

Kurt Schaefer

Die »bibliothekarische Kränkung«. Der Weg von einer Abteilung der

Hauptbibliothek hin zum Zentralen Service Team Sacherschließung . . . 63

Markus Stumpf

Erinnerungsarbeit, Restitution und historische Verantwortung.

Ein Überblick zum Arbeitsbereich NS-Provenienzforschung . . . . . . . . 67

Viktoria Lang-Steixner

Was ein ideales Bibliothekssystem können sollte. Die wechselvolle Arbeit der Arbeitsgruppe Alma . . . . . . . . . . . . . . . 71

Birgit Kopar / Stefan Wiborny

Alle Zweigstellen unter einem (Koordinations-)Hut. Permanente

Arbeitsgruppe Benutzung . . . . . . . . . . . . . . 7

Karin Lach

Die Universitätsbibliothek als Informationskompetenzzentrum der Universität. Permanente Arbeitsgruppe Teaching Library . . . . . . . . . 79

\section{Archiv}

Thomas Maisel

Denn was zusammen gehört, findet auch zusammen? Das

Universitätsarchiv als Teil der $»$ Dienstleistungseinrichtung Bibliotheks-

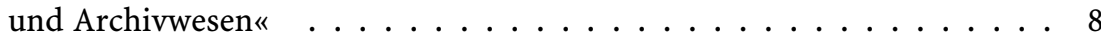




\section{Hauptbibliothek}

Pamela Stückler

There and Back Again ... Die Abteilung Alte und wertvolle Bestände . . . 91

Christine Bauer

Ein bisschen Werbung für die Erwerbung. Die Abteilung Bestellung und

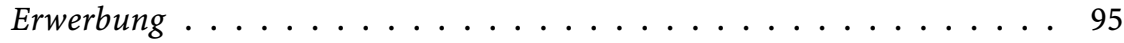

Birgit Kopar / Helmut Maißer / Stefan Wiborny

Die ELFe der Hauptbibliothek. Die Abteilungen Entlehnung und

Lehrbuchsammlung und Fernleihe . . . . . . . . . . . . . . . . . . 99

Elisabeth Schmid / Kerstin Edlinger

Formalerschließung von A bis Z. Universitätsbibliothek /

Hauptbibliothek / Abteilung Formalerschließung . . . . . . . . . . . . 107

Sandra Mann

Wohin mit all den Fragen? Team Infodienst und Führungen . . . . . . . . 111

\section{Fachbereichsbibliotheken}

Birgit Athumani Hango

maktaba yangu - مكتبتي - ọá akwụkwọ m - benim kütüphanem -

كتابخانه من Die Fachbereichsbibliothek Afrikawissenschaften und

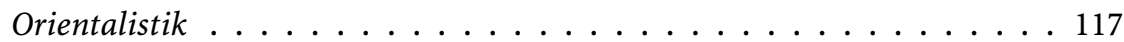

Andrea Ramharter-Hanel

Nicht von Gestern. Die Fachbereichsbibliothek Alte Geschichte . . . . . . 121

Karin Lach

Same same or different? Die Fachbereichsbibliothek Anglistik und

Amerikanistik 2004-2019: Vor der Bibliothek muss man sich nicht

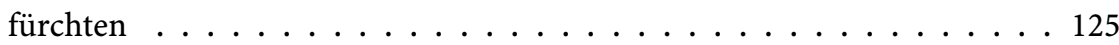

Sandra Zoglauer

Eine Bibliothek mit Weitblick. Die Fachbereichsbibliothek Archäologien

und Numismatik . . . . . . . . . . . . . . . . . . . . . . . . 129

Manfred Schattleitner

Dem Himmel so nah. Die Fachbereichsbibliothek Astronomie . . . . . . . 133 
Ariella Sobel

Die Universitätsbibliothek kommt ihrem Ziel der Einschichtigkeit wieder ein Stück näher. Die Fachbereichsbibliothek Bildungswissenschaft, Sprachwissenschaft und Vergleichende Literaturwissenschaft . . . . . . 135

Eva-Maria Mikschi-Marischler

Auch eine Frage des Standortes. Die Geschichte der

Fachbereichsbibliothek Biologie . . . . . . . . . . . . . . 139

Eva-Maria Mikschi-Marischler / Matthias Svojtka

Blüten, Bücher, Bytes - Systematische Botanik im digitalen Zeitalter.

Die Fachbereichsbibliothek Botanik . . . . . . . . . . . . . . . . . . . . 143

Anna Ransmayr

Die neue Bibliothek in der Alten Universität. Die Fachbereichsbibliothek

Byzantinistik und Neogräzistik . . . . . . . . . . . . . . 147

Manfred Schattleitner

Millionen Jahre Erdgeschichte. Die Fachbereichsbibliothek

Erdwissenschaften und Meteorologie . . . . . . . . . . . . . . 151

Susanne Wicha

War das Karteikärtchen weiblich, weil auch »dienlich«? Von der

Zettelwirtschaft an der Fachbereichsbibliothek Europäische Ethnologie . . 155

Alexandra Gappmayr

Retro-Charme und neue Maßstäbe. Der Fachbereichsbibliothek

Geographie und Regionalforschung in die Karten geschaut . . . . . . . . 159

Stefan Alker-Windbichler

Dieses gesunde grüne Leuchten. Die Fachbereichsbibliothek Germanistik, Nederlandistik und Skandinavistik als Bibliotheksraum . . . . . . . . . 161

Harald Tersch

Ein bibliothekarisches Experiment. Die Fachbereichsbibliothek

Geschichtswissenschaften .................. 165

Monika Schreiber

Von der Festung zum Aktionsraum. Die Fachbereichsbibliothek Judaistik im Wandel . . . . . . . . . . . . . . . . . . . . . . . . . . . 169 
Sonja Reisner

Nicht nur ein Ort des stillen Studiums alter Texte.

Die Fachbereichsbibliothek Klassische Philologie, Mittel- und Neulatein 173

Birgit Kramreither

Sammeln ist unsere Leidenschaft (Daten, Objekte, Menschen).

Die neuen Herausforderungen der Fachbereichsbibliothek Kultur- und

Sozialanthropologie . . . . . . . . . . . . . . 177

Martin Steinreiber

Mit 167 Jahren in die Zukunft. Die Fachbereichsbibliothek

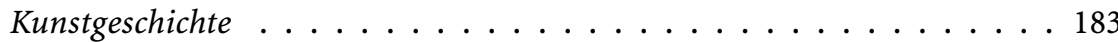

Benedikt Lodes

Small is beautiful. Die Fachbereichsbibliothek Musikwissenschaft . . . . . 187

Gabriele Pauer

Keine Außenseiter mehr. Die Fachbereichsbibliothek

Ostasienwissenschaften . . . . . . . . . . . . . . . 191

Barbara Retschnig

Eine neue Bibliothek - zwei alte Wurzeln. Die Fachbereichsbibliothek

Osteuropäische Geschichte und Slawistik . . . . . . . . . . . . . . 195

Kurt Schneider

Arzneistoffe, Arzneipflanzen, Public Health - Von der Inkunabel zur

Online-Suchmaschine. 25 Jahre Fachbereichsbibliothek Pharmazie und

Ernährungswissenschaften im Universitätszentrum Althanstraße . . . . . 199

Sonja Fiala

Vor Gericht und auf hoher See ... Die Fachbereichsbibliothek Philosophie 203

Michaela Zemanek

„Die Bibliothek ist eine Lernstätte, nicht ein Lager für Bücher«.

Die Fachbereichsbibliothek Psychologie . . . . . . . . . . . . . . 207

Tanja Fabian

»Kaschmir«-Parkett und orange Kompaktanlagen.

Die Fachbereichsbibliothek Publizistik- und Kommunikationswissenschaft

und Informatik . . . . . . . . . . . . . . . . 211 
Thomas Luzer

Bibliotheca semper reformanda. Oder warum sich an der

Fachbereichsbibliothek Rechtswissenschaften so viel verändert hat . . . 215

Christina Köstner-Pemsel

Bibliothécaire/bibliotecaria/bibliotecária/bibliotecară. Bibliothekarinnen an der Fachbereichsbibliothek Romanistik . . . . . . . . . . . . . . . . . 219

Beate Lang

"Ich mag meine Fachbereichsbibliothek«. Empathie, Diskurs und

Forschungsinteresse an der Fachbereichsbibliothek Soziologie und

Politikwissenschaft .................... 223

Gerda McNeill

Eine Transformation. Von der Institutsbibliothek zur

Fachbereichsbibliothek Sportwissenschaft . . . . . . . . . . . . . . 227

Martina Cuba

Desperately seeking library relocation. Ein neuer Standort für die

Fachbereichsbibliothek Theater-, Film- und Medienwissenschaft . . . . 231

Alfred Friedl

El universo (que otros llaman la Biblioteca) ...

Die Fachbereichsbibliothek Theologie . . . . . . . . . . . . . . 235

Andrea Neidhart

Aus 5 mach 1 - bibliothekarisches »Hexen-Einmal-Eins «? Die neue

Fachbereichsbibliothek Wirtschaftswissenschaften und Mathematik . . . . 239

Markus Stumpf

Die »Videosammlung Stadtkino« als Teil des österreichischen

televisuellen Gedächtnisses an der Fachbereichsbibliothek Zeitgeschichte . 243

\section{Forschungsunterstützende Services und Projekte}

Alexander Sperl

Der Film in der Welt des Buches. Die $A G A V$-Medien im Unterricht an der Universitätsbibliothek Wien . . . . . . . . . . . . 251 
Juan Gorraiz / Christian Gumpenberger / Ursula Ulrych /

Martin Wieland

Mehr als nur Erbsenzähler und Kaffeesudleser. Die Abteilung

Bibliometrie und Publikationsstrategien im Zeitalter von "publish or

perish" und Open Science . . . . . . . . . . . . . . . . 253

Lars Kaczmirek

Der erfolgreiche Aufbau einer nationalen Dateninfrastruktur. AUSSDA -

The Austrian Social Science Data Archive . . . . . . . . . . . . . 257

Claudia Feigl

Der Umgang mit Objekten im Rahmen von Wissenschaft und Lehre.

Die Sammlungskoordinierungsstelle der Universität Wien . . . . . . . . 261

Guido Blechl

Open Access: Von den Anfängen bis heute . . . . . . . . . . . . 265

Susanne Blumesberger

"Druckt ihr eh alles aus was in PHAIDRA ist?" Die Abteilung

Repositorienmanagement PHAIDRA-Services - ein Paradigmenwechsel . 269

Manuela Rohrmoser

Der ganz normale Wahnsinn und manchmal sogar ein Krimi.

ubw:helpdesk und User Training . . . . . . . . . . . . . . . . 273

Michael Greil

»Ihr seid's ja nicht von der UB, ihr seid's von der Uni!« Institutioneller

Wandel illustriert anhand der Einführung des

Forschungsinformationssystems $u$ :cris . . . . . . . . . . . 277

Martin Gasteiner

NewsEye. Ein Horizon 2020-Projekt an der Universitätsbibliothek Wien . 283

Gerda McNeill

Die Universitätsbibliothek Wien als Partnerin europäischer Projekte.

Von den Anfängen bis OpenAIRE Advance . . . . . . . . . . . . . 287 


\section{Gastbeiträge}

Andreas Brandtner

Wiener Lektionen. Rückblicke auf die Organisationsentwicklung der

Universitätsbibliothek Wien . . . . . . . . . . . . . . . 293

Claudia Hausberger

Lessons learned . . . . . . . . . . . . . . . . . . . . . . . . . 299

Ute Wödl

Strategisch denken, Veränderung ermöglichen, Teamarbeit unterstützen und individuelle Entwicklung fördern - Gelebte Grundprinzipien in der

Bibliotheksleitung bei Maria Seissl . . . . . . . . . . . . . . 303

\section{Zugabe}

Benedikt Lodes

Der UB-Chor. Eine Plattform für inneren Ausgleich und innerbetriebliche

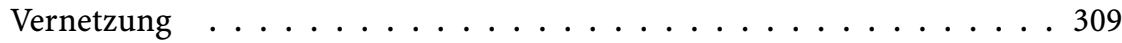

Impressionen aus den Jahren 2004-2019

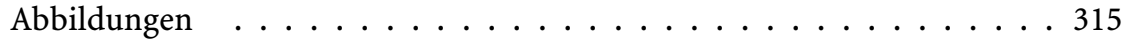

\section{Anhang}

Autorinnen und Autoren . . . . . . . . . . . . . . . 331 


\section{Regina Hitzenberger}

\section{Grußwort}

Herzliche Gratulation zum 60. Geburtstag von Fr. HRin Mag. Maria Seissl und zum 15-jährigen Jubiläum ihrer Leitung der Dienstleistungseinrichtung (DLE) Bibliotheks- und Archivwesen!

In meiner Funktion als Vizerektorin für Infrastruktur an der Universität Wien kann ich bereits auf fünf Jahre sehr guter Zusammenarbeit mit Maria Seissl zurückblicken. Ihre breite Fachkenntnis, ihre Zielorientiertheit und ihr partizipativer, auf breiten Konsens orientierter Führungsstil haben die DLE geprägt und erfolgreich durch diese Jahre geführt.

Die DLE Bibliotheks- und Archivwesen erfuhr unter ihrer Leitung grundlegende Wandlung in zentralen Bereichen. Die Einführung von eRessourcen mit der Zielsetzung »Elektronisch wo möglich, Print wo nötig", die Digitalisierung von Bibliotheksressourcen, die Einführung von forschungsunterstützenden Services wie z. B. dem Forschungsinformationssystem u:cris, dem Repositorium PHAIDRA und der Bibliometrie waren große Schritte der DLE in die digitale Welt. Nicht mehr der Besitz von Informationsträgern, sondern die allgemeine Zugänglichkeit von Information steht nun im Vordergrund. Im Bereich der physischen Bibliothek wurden Standorte zusammengelegt und neu gestaltet, wodurch ein besseres Service für Wissenschafter_innen und Studierende ermöglicht wird, was mir besonders wichtig ist.

Im Namen des Rektorats der Universität Wien danke ich Maria Seissl und ihrem Team für ihr Engagement und freue mich auf eine weiterhin erfolgreiche Zusammenarbeit auf dem Weg zur Bibliothek der Zukunft! 
Open-Access-Publikation im Sinne der CC-Lizenz BY-NC-ND 4.0

(c) 2019, Vandenhoeck \& Ruprecht GmbH \& Co. KG, Göttingen ISBN Print: 9783847110989 - ISBN E-Lib: 9783737010986 


\section{Vorwort}

Maria Seissl feierte im Jahr 2019 einen runden Geburtstag und kann gleichzeitig auf 15 Jahre als Leiterin der Universitätsbibliothek Wien zurückblicken. Diese beiden Jubiläen boten eine besonders günstige Gelegenheit für die Herausgabe einer Festschrift, die nicht nur die Absicht verfolgt, auf die Ereignisse der letzten 15 Jahre an der Universitätsbibliothek Wien zurückzublicken, sondern auch die aktuelle Situation festzuhalten, in der sich die Bibliothek momentan befindet. Bewusst sollte die zwischenzeitlich zur Dienstleistungseinrichtung (DLE) ausgebaute Organisationseinheit »UB Wien« in ihrer Vielfalt und Vielstimmigkeit abgebildet werden, weshalb alle Leiterinnen und Leiter sowohl der Fachbereichsbibliotheken, als auch der Abteilungen und Arbeitsgruppen eingeladen wurden, einen Aufsatz für diese Festschrift beizusteuern.

Mit dieser partizipativen Herangehensweise und der Idee, das Werk in diesem Umfang herauszubringen, sollte nicht zuletzt dem persönlichen Führungsstil Maria Seissls Rechnung getragen werden, mit dem sie die Bibliothek in den letzten 15 Jahren umgestaltet und die Dienstleistungseinrichtung Bibliotheksund Archivwesen aufgebaut hat: Größtmögliche Einbindung der Mitarbeiterinnen und Mitarbeiter, mit dem Bestreben, einen möglichst breiten Konsens herbeizuführen.

Der Titel Menschen im Aufbruch (kurz: MIA) stellt die Menschen in den Mittelpunkt und macht deutlich, dass diese Festschrift die DLE Bibliotheks- und Archivwesen nicht über ihre Funktionen beschreiben möchte, sondern durch jene Menschen, die diese Einrichtung in ihrer täglichen Arbeit prägen und mitgestalten. Darüber hinaus möchte der Titel auf die Offenheit und Dynamik hinweisen, mit der die Jubilarin die Bibliothek in den letzten eineinhalb Jahrzehnten leitet: Vieles musste sich ändern, um den allgemeinen Entwicklungen zu folgen, vieles konnte sich aber auch zusätzlich entwickeln, weil sie als Leiterin der Kreativität ihrer Mitarbeiterinnen und Mitarbeiter Raum gegeben und deren Innovationskraft gefördert hat.

Die Auswahl der Autorinnen und Autoren für diese Festschrift folgte streng dem offiziellen Organigramm der Dienstleistungseinrichtung. Aufgrund der 
bestehenden flachen Hierarchie umfasst die zweite Führungsebene mehr als 70 Personen und sie alle kommen in dieser Festschrift zu Wort. In den (Unter-)Titeln der jeweiligen Beiträge wird der Verantwortungsbereich bzw. der Name der Fachbereichsbibliothek oder Abteilung klar ersichtlich. Neben dieser Fülle an Einblicken gibt es vier weitere Beiträge, die jenseits der Organisationsstruktur Aufnahme in diesen Band gefunden haben:

Zunächst sind dies drei Beiträge von ehemaligen Mitarbeiter_innen, die mittlerweile selbst als Leiterinnen bzw. Leiter von Bibliotheken tätig sind und die wir gebeten haben, auf ihre Zeit an der Universitätsbibliothek Wien zurückzublicken: Andreas Brandtner, Direktor der Universitätsbibliothek der Freien Universität Berlin, Claudia Hausberger, Leiterin der Universitätsbibliothek der Veterinärmedizinischen Universität Wien und Ute Wödl, Leiterin der AK Bibliothek Wien für Sozialwissenschaften.

Der letzte dieser Aufsätze ist dem UB-Chor gewidmet, der in der Ära Maria Seissl entstanden ist und der aus unserer Sicht paradigmatisch für ihre Grundanliegen steht: Kolleginnen und Kollegen über Abteilungsgrenzen hinweg zusammenzubringen, einen Rahmen für Kommunikation und Austausch anzubieten und den Zusammenhalt untereinander zu stärken. Gerade der Chor macht bewusst, dass man als einzelne Mitarbeiterin bzw. Mitarbeiter einem größeren Ganzen angehört. Und dass sich jede bzw. jeder - wie beim Musizieren - zwar einerseits mit seiner Stimme einbringt, man dabei aber gleichzeitig aufeinander hören muss, um ein gemeinsames Ganzes entstehen zu lassen.

In diesem Sinne und im Namen der gesamten Organisationseinheit: Alles Gute, liebe MIA!

Wien 2019 


\section{Einführung: Die Dienstleistungseinrichtung Bibliotheks- und Archivwesen im Überblick}

Maria Seissl trat mit 1. Januar 2000 in die Dienste der Universitätsbibliothek Wien, nachdem sie in Innsbruck Anglistik studiert, in den 1980er Jahren an der Universitäts- und Landesbibliothek Innsbruck das bibliothekarische Handwerkszeug erlernt und sich in der Folgezeit einen Namen im Bibliothekswesen gemacht hat, insbesondere in der bibliothekarischen Ausbildung. Bereits in den 1990er Jahren fungierte sie als Vorsitzende der Prüfungskommission für den Bibliotheksdienst.

So sehr der Wechsel von Innsbruck nach Wien für Maria Seissl eine große Veränderung bedeutete, so sehr brachte dieses Jahr für die Universitätsbibliothek Wien eine grundlegende Neuerung in der Bibliotheksverwaltung mit sich: Denn mit 1. Januar 2000 wurde an der Universität Wien das Universitätsorganisationsgesetz (UOG) 1993 wirksam. Die Universitätsbibliotheken wurden mit diesem Gesetz als Dienstleistungseinrichtungen der jeweiligen Universität definiert und unmittelbar der Universitätsleitung unterstellt. Was heute eine Selbstverständlichkeit ist - und jüngere Leserinnen und Leser werden sich fragen, wie es je anders organisiert gewesen sein konnte - war damals aber so neu, dass man damit erst umzugehen lernen musste. Dem UOG 1993 kann ohne Übertreibung attestiert werden, einen Paradigmenwechsel herbeigeführt zu haben. Vor dieser Neuorganisation galt für Universitätsbibliotheken nämlich jahrhundertelang ein System, das dem Geist des aufgeklärten Absolutismus verpflichtet war, seine Wurzeln in der Reform des Hochschulwesens des 18. Jahrhunderts hatte und namentlich auf Maria Theresia und Joseph II. zurückzuführen ist. In einer gänzlich anderen Logik als heute unterstanden die Universitätsbibliotheken direkt der zuständigen staatlichen Behörde. ${ }^{1}$ Alle Verwaltungsabläufe, alle Budget- und Personalfragen, aber auch alle bibliothekarischen Aktivitäten waren ehemals mit der staatlichen Behörde abzustimmen. So

1 Das Bundesministerium für Wissenschaft und Forschung, dessen Bezeichnung und konkreter Zuständigkeitsbereich im Zuge neuer Regierungskonstellationen wechselte, war bis Jahresende 1999 für die Universitätsbibliothek unmittelbar zuständig. 
wenig man sich heute vorstellen kann, dass Universitätsbibliotheken nicht direkt der Universität zugeordnet sind, so selbstverständlich war es in den mehr als zweihundert dem UOG 1993 vorausgegangenen Jahren, dass Universitätsbibliotheken der staatlichen Behörde unterstehen. Ferdinand Grassauer (1840-1903), der im Jahr 1884 die Übersiedlung der Universitätsbibliothek Wien von der Postgasse an den heutigen Standort an der Ringstraße vornahm und von diesem Jahr an bis 1903 als Bibliotheksdirektor wirkte, schreibt in seinem 1883 erstmals erschienenen Handbuch für österreichische Universitäts- und StudienBibliotheken mit größter Selbstverständlichkeit:

Die Universitäts- und Studienbibliotheken sind selbständige Staatsanstalten, welche in den Wirkungskreis des k. k. Ministeriums für Cultus und Unterricht gehören, und der Statthalterei, beziehungsweise Landesregierung des Kronlandes, in welchem sie sich befinden, unmittelbar untergeordnet sind. ${ }^{2}$

Mit Jahresanfang 2000 ist an der Universitätsbibliothek Wien diese jahrhundertelang geübte, gelebte und vertraute Praxis Geschichte. Die Bibliothek betritt Neuland. Die Zusammenarbeit mit der Universitätsleitung als nunmehr für die Bibliothek unmittelbar vorgesetzte Stelle will gestaltet sein. In dieser historischen Stunde tritt Maria Seissl ihren Dienst an der Universitätsbibliothek Wien an. Als Zweite Stellvertreterin der damaligen Bibliotheksdirektorin Ilse Dosoudil ist sie Mitglied des damals dreiköpfigen Leitungsteams. Sie beginnt proaktiv, diese Beziehungen mitzugestalten. Mit ihrer gewinnenden Art und ihrer Differenzen ausgleichenden Grundhaltung entwickelt sich rasch ein produktives Miteinander von Universitätsleitung und Bibliotheksleitung.

In ihrem ersten Dienstjahr ist Maria Seissl gleichzeitig für die Hauptabteilung II »Bearbeitung und Information« zuständig. ${ }^{3}$ Mit Februar 2001 rückt Maria Seissl in die Position der Ersten Stellvertreterin der Bibliotheksdirektorin auf und wird gleichzeitig für die Hauptabteilung IV, den Dezentralen Bereich, verantwortlich. In den Hauptabteilungen I und III fungiert sie als stellvertretende Leiterin. Mit diesem Portfolio an Aufgaben hat Maria Seissl Einblick in und gestalterischen Einfluss auf sämtliche Bereiche der Universitätsbibliothek Wien. Als die Bibliotheksdirektorin Ilse Dosoudil im Jahr 2004 ihren Ruhestand antritt, betraut die Universitätsleitung Maria Seissl - zunächst interimistisch - mit der

2 Ferdinand Grassauer: Handbuch für österreichische Universitäts- und Studien-Bibliotheken sowie Volks-, Mittelschul- und Bezirks-Lehrerbibliotheken. Wien: Graeser 1883, S. 40.

3 Die Universitätsbibliothek Wien war damals in vier Hauptabteilungen untergliedert: Hauptabteilung I = Bestandsaufbau, Hauptabteilung II = Bearbeitung und Information, Hauptabteilung III = Benützung, Hauptabteilung IV = Dezentraler Bereich (das sind Fakultätsbibliotheken, Fachbibliotheken und sogenannte Institutsaufstellungen). Der Direktion, die damals aus der Bibliotheksdirektorin und zwei Stellvertreterinnen bestand, waren in dieser Zeit fünf Stabsstellen zugeordnet, nämlich die Bereiche Ausbildung, Systembibliothekare, ADV, Verwaltung und Personalreferat. 
Leitung der Universitätsbibliothek Wien. Mit 1. Januar 2005 wird sie formal zur Leiterin der Dienstleistungseinrichtung bestellt. ${ }^{4}$

$\mathrm{Ab}$ dieser Zeit wird die Dienstleistungseinrichtung organisatorisch umgestaltet. Besonders deutlich machen sich die Veränderungen im sogenannten "Außenbereich« bemerkbar: Nachdem die Zentralbibliothek für Medizin der aus der Medizinischen Fakultät entstandenen Medizinischen Universität Wien zugeordnet war, wurden die verbleibenden Fakultätsbibliotheken sowie die Fachbibliotheken zu Fachbereichsbibliotheken. Im Zuge dieser Umstellungen wurden nach und nach auch Institutsbibliotheken, die zuvor personell und organisatorisch nicht Teil der Dienstleistungseinrichtung waren, der Universitätsbibliothek eingegliedert. Im Laufe der Jahre konnten bei dieser Strukturbereinigung auch Standorte reduziert werden. Die markantesten Änderungen in diesem Zusammenhang sind die Zusammenlegung der theologischen Bibliotheken im Jahr 2007 in der Schenkenstraße 8, die Integration der Fachbereiche Bildungswissenschaft, Sprachwissenschaft und Vergleichende Literaturwissenschaft in eine gemeinsame Fachbereichsbibliothek am neu errichteten Standort Sensengasse 3 im Jahr 2010, die Eröffnung der Fachbereichsbibliothek Publizistik- und Kommunikationswissenschaft und Informatik im Neubau Währinger Straße 29 im Jahr 2012 sowie die Zusammenführung der zuvor getrennten Standorte Volkswirtschaftslehre und Betriebswirtschaftslehre der Fachbe-

4 Seit dem Jahr 2004 ist die Dienstleistungseinrichtung auch für das Archiv der Universität Wien zuständig und trägt seither die Bezeichnung »Dienstleistungseinrichtung (DLE) Bibliotheksund Archivwesen«. Seit diesem Zeitpunkt wird der Begriff »Universitätsbibliothek« für zwei Entitäten verwendet: Im herkömmlichen und weiten Sinn bezeichnet er an der Universität Wien die Summe der bibliothekarischen Einrichtungen, also die Hauptbibliothek und die Fachbereichsbibliotheken (inklusive der vornehmlich nach innen gerichteten Zentralen Services) oder mit anderen Worten all jene Abteilungen, die es an der Dienstleistungseinrichtung gibt, wenn man das Archiv der Universität Wien ausklammert, also insgesamt das "Bibliothekswesen «. Der Organisationsplan der Universität Wien - 2004 ist erstmals ein solcher auf Basis des UG 2002 erschienen - kennt ausschließlich den Begriff »Bibliotheks- und Archivwesen«, eine Bezeichnung »Universitätsbibliothek« für den Teil »Bibliothekswesen« kennt der Organisationsplan nicht. Um den Begriff aber weiterhin offiziell aufscheinen zu lassen - auf internationaler Ebene ist "Universitätsbibliothek" die bis heute übliche Bezeichnung -, wird der Begriff »Universitätsbibliothek« in einem engen Sinn seit 2004 für die Hauptbibliothek bzw. alle bibliothekarischen Bereiche abzüglich der Fachbereichsbibliotheken verwendet. Alle Bibliotheksmitarbeiterinnen und -mitarbeiter, die keiner Fachbereichsbibliothek zugeordnet sind, werden im Personalverzeichnis der "Universitätsbibliothek" zugeordnet. Die Binnenstruktur der Dienstleistungseinrichtung Bibliotheks- und Archivwesen kennt als echte Subeinheiten nämlich bis dato nur die Fachbereichsbibliotheken, das Archiv, die Core Facility AUSSDA sowie die »Universitätsbibliothek«. Es zeichnet sich ab, dass in naher Zukunft alle Abteilungen der DLE zu echten Subeinheiten werden. Wie es der Zufall will, befindet sich das Organigramm genau zum Zeitpunkt des Erscheinens dieser Festschrift in Überarbeitung. Im Illustrationsteil des Bandes werden sowohl das in den letzten Jahren gültige Organigramm (das für die Gliederung der Festschrift strukturgebend war) als auch der Entwurf des gerade neu erarbeiteten Organigramms abgedruckt. 
reichsbibliothek Wirtschaftswissenschaften und ihre Kombination mit dem Fachbereich Mathematik am Standort Oskar-Morgenstern-Platz 1 im Jahr 2013.

Die Bibliothek Sportwissenschaft wurde organisatorisch und personell im Jahr 2013 Teil der Dienstleistungseinrichtung, die ehemalige Institutsbibliothek Ägyptologie kam mit Übersiedlung an den Standort der Fachbereichsbibliothek Archäologien und Numismatik im Jahr 2014 organisatorisch zur Universitätsbibliothek. 2016 wurde schließlich die Bibliothek des Instituts für Österreichische Geschichtsforschung der Universitätsbibliothek Wien zugeordnet.

Mit dem Wandel des Außenbereichs ging eine Umstrukturierung der Aufbauorganisation Hand in Hand: Die Hauptabteilungen wurden abgeschafft, dafür aber die nach innen gerichteten Services als sogenannte Zentrale Services definiert. Die Hierarchie wurde flacher, die Führungsspanne größer: Maria Seissl ist direkte Vorgesetzte der neun Abteilungsleiterinnen und -leiter der Hauptbibliothek, der vierzehn Leiterinnen und Leiter der Zentralen Services sowie der im Laufe der Jahre entstandenen neun Forschungsunterstützenden Services, von denen in diesem Überblick noch zu berichten sein wird, des Archivleiters, des stellvertretenden DLE-Leiters und der Leiter_innen temporär eingerichteter Drittmittelprojekte. Die stellvertretende DLE-Leitung ist für die 38 Fachbereichsbibliotheken ${ }^{5}$ die direkt vorgesetzte Stelle. ${ }^{6}$

Aber nicht nur die Verwaltungsabläufe befanden sich im Umbruch, auch die Informationsträger selbst und die Art und Weise ihres Nachweises und ihrer Auffindbarkeit begannen sich grundlegend zu wandeln. Während bereits seit den ausgehenden 1980er Jahren Katalogisate sukzessive in einem elektronischen Bibliothekssystem erstellt und damit online zugänglich wurden, haben sich unter Maria Seissl neue Bereiche, Projekte und internationale Kooperationen ergeben, die in dieser Festschrift als eigenständige Beiträge zur Sprache kommen, auf die aber bereits in der Einführung zusammenfassend hingewiesen werden soll: Das hauseigene Repositorium PHAIDRA ${ }^{7}$ wurde entwickelt, das mittlerweile in fünf Ländern an mehr als einem Dutzend Partnerinstitutionen eingesetzt wird. Ein Forschungsdokumentations- und -informationssystem ${ }^{8}$, die Arbeitsbereiche Bibliometrie und Szientometrie ${ }^{9}$, Open Access ${ }^{10}$ sowie ein So-

\footnotetext{
5 Mit September 2019 reduzierte sich die Zahl der Fachbereichsbibliotheken auf 37, da die FB Philosophie und die FB Psychologie organisatorisch zu einer Fachbereichsbibliothek zusammengelegt wurden.

6 2004/2005 wurde die Position einer/eines Zweiten Stellvertreters/in abgeschafft, sodass es seit damals nur noch die Position einer/eines stellvertretenden DLE-Leiter/in gibt.

7 Siehe Beitrag zu PHAIDRA von Susanne Blumesberger.

8 Siehe Beitrag zu $u$ :cris von Michael Greil.

9 Siehe Beitrag Bibliometrie und Publikationsstrategien von Juan Gorraiz, Christian Gumpenberger, Ursula Ulrych und Martin Wieland.

10 Siehe Beitrag zum Open Access Office von Guido Blechl.
} 
zialwissenschaftliches Datenarchiv ${ }^{11}$ wurden etabliert. Bei der Versorgung mit elektronischem Informationsangebot entwickelte sich im Laufe der Direktion Maria Seissls die Notwendigkeit, eigene Arbeitsbereiche für eResource Management und konsortiale Erwerbung ${ }^{12} \mathrm{zu}_{\text {schaffen. Auch eine Webredaktion }}{ }^{13}$ und die Abteilung für Öffentlichkeitsarbeit ${ }^{14}$ sowie eine Stelle, die sich unmittelbar mit Innovation ${ }^{15}$ befasst, entwickelten sich aufgrund des sich verändernden Umfelds; die Bedeutung des Teams Bibliothekssysteme und des Teams EDV-Infrastruktur wurde gewichtiger; ein weitgehend virtueller Helpdesk für die gesamte Universitätsbibliothek ${ }^{16}$ sowie eine eigene Arbeitsgruppe für Teaching Library ${ }^{17}$ ergänzten die traditionellen Arbeitsbereiche. Außergewöhnlich und im zentraleuropäischen Raum als Best-Practice-Modell angesehen ist die Einrichtung einer Sammlungskoordinierungsstelle und ihre Verankerung im Bibliotheks- und Archivwesen. ${ }^{18}$ Ähnlich richtungsweisend ist die Einrichtung eines permanenten Arbeitsbereichs für NS-Provenienzforschung zumindest für die österreichische Bibliothekslandschaft. ${ }^{19}$ Neben all diesen neuen Bereichen engagiert sich die Universitätsbibliothek Wien unter Leitung von Maria Seissl teils federführend in nationalen und internationalen Projekten, die mit Openness zu tun haben.

Wenn sich diese einzelnen Bereiche in dieser Festschrift auch selbständig vorstellen, wurden sie hier bereits kursorisch erwähnt. Und das nicht etwa, um sie gegenüber den traditionellen Arbeitsbereichen der Bibliothek und des Archivs hervorzuheben, die in dieser Festschrift ebenfalls ausführlich zu Wort kommen, sondern weil sich die genannten neuen Bereiche unter der Leitung Maria Seissls etabliert haben bzw. genauer gesagt von ihr etabliert wurden. Dass sich diese Bereiche überhaupt in einer solchen Breite an der Universitätsbibliothek Wien entwickeln konnten, legt eine Grundintention Maria Seissls offen: Die Bibliothek als zukunftsfähigen Dreh- und Angelpunkt in der wissenschaftlichen Informationsversorgung zu positionieren, als echte Partnerin von Forschung, Lehre und Studium.

Dieser von Maria Seissl bewusst gesetzte Zugang und ihr diesen Entwicklungen zugrundeliegendes Verständnis eines wissenschaftlichen Bibliothekswesens auf der Höhe der Zeit hat gleichzeitig Dynamik im Personaleinsatz er-

11 Siehe Beitrag Core Facility AUSSDA von Lars Kaczmirek.

12 Siehe Beitrag E-Resource Management und Clearingstelle Konsortien von Brigitte Kromp und Wolfgang Mayer.

13 Siehe Beitrag Webredaktion von Horst Prillinger.

14 Siehe Beitrag Öffentlichkeitsarbeit von Pamela Stückler.

15 Siehe Beitrag ubw:innovation von Adelheid Mayer.

16 Siehe Beitrag ubw:helpdesk und User Training von Manuela Rohrmoser.

17 Siehe Beitrag AG Teaching Library von Karin Lach.

18 Siehe Beitrag Sammlungskoordinierungsstelle der Universität Wien von Claudia Feigl.

19 Siehe Beitrag Arbeitsbereich NS-Provenienzforschung von Markus Stumpf. 
möglicht: Während sich ihr schon erwähnter Vorgänger Ferdinand Grassauer Ende des 19. Jahrhunderts erfolgreich für die Systemisierung von Bibliothekarsstellen eingesetzt hat - ein Meilenstein in der Professionalisierung des universitären Bibliothekswesens -, ist es Maria Seissl im beginnenden 21. Jahrhundert - in einer Zeit deutlichen Drucks in Richtung Personalreduktion im öffentlichen Dienst - gelungen, für junge Menschen in der Bibliothek neue Aufgabenfelder zu erschließen und so Arbeitsplätze zu sichern. Während konkrete Personaleinsparungsaufträge des Rektorats vereinbarungsgemäß umgesetzt wurden ${ }^{20}$, ist es gleichzeitig durch Erschließung neuer Tätigkeitsfelder und die nachhaltige Sicherung ihrer Ausstattung mit Personal möglich gewesen, dass die Personalreduktion an der Dienstleistungseinrichtung nicht als harter Aderlass wahrgenommen worden ist.

Evaluationen in den Jahren 2010 und 2018 sowie regelmäßige Benutzer_innenumfragen bestätigten und bestätigen den Kurs, auf dem Maria Seissl die Dienstleistungseinrichtung Bibliotheks- und Archivwesen der Universität Wien zu neuen Horizonten führt. Mit der vorliegenden Festschrift bedankt sich die zweite Führungsebene stellvertretend für alle Mitarbeiterinnen und Mitarbeiter der Dienstleistungseinrichtung bei MIA Seissl, sich als »Menschen im Aufbruch" und in einem Arbeitsumfeld wissen zu dürfen, das beruflich erfüllt und das in einer sich rasch verändernden Welt einen positiven Beitrag zum großen Ganzen leistet.

202010 sowie 2017 gab es konkrete Personaleinsparungsaufträge durch Nichtnachbesetzung bei Pensionierung mit mehrjährigen Laufzeiten. 
Zentrale Services und Arbeitsgruppen 
Open-Access-Publikation im Sinne der CC-Lizenz BY-NC-ND 4.0

(c) 2019, Vandenhoeck \& Ruprecht GmbH \& Co. KG, Göttingen ISBN Print: 9783847110989 - ISBN E-Lib: 9783737010986 


\section{Wo die Fäden zusammenlaufen. Das Büro Bibliotheks- und Archivwesen als zentrale Clearingstelle für Verwaltung und Finanzen}

Als Maria Seissl ihren Dienst an der Universitätsbibliothek Wien antrat, funktionierte die Verwaltung in einer ganz anderen Weise als heute: Die Universitätsbibliothek war direkt dem damaligen Bundesministerium für Wissenschaft und Forschung unterstellt; alle finanziellen Mittel von der Büroausstattung über die Literaturanschaffung bis hin zu Verbrauchsmaterialien mussten dort beantragt und verhandelt werden.

Mit Jahresbeginn 2000 trat für die Universität Wien das Universitätsorganisationsgesetz 1993 in Kraft. Dadurch wurde die Bibliothek eine der Universitätsleitung direkt unterstellte Dienstleistungseinrichtung. Für die Verwaltung war das ein Paradigmenwechsel. Nicht nur der institutionelle Ansprechpartner wechselte, auch die Unternehmenskultur veränderte sich grundlegend. In dieser Übergangsphase gestaltete Maria Seissl als stellvertretende Direktorin den Wandel mit und zeichnete für die Implementierung neuer Abläufe ab 2004 verantwortlich. Mit großem Geschick gelang es ihr, für die Dienstleistungseinrichtung einen Modus $\mathrm{zu}$ etablieren, der innerhalb definierter Budgetblöcke größeren Spielraum ermöglicht. Das brachte auch Veränderungen für die Abteilung, die im Zentrum der Verwaltung der Dienstleistungseinrichtung steht das Büro Bibliotheks- und Archivwesen, das heute für ein sehr breites Aufgabenspektrum verantwortlich ist:

\section{Direktionsbüro}

Das Direktionsbüro unterstützt die alltägliche Arbeit der Leitung, koordiniert Terminanfragen, bearbeitet Korrespondenz und registriert die bearbeiteten Schriftstücke. Die Vergabe von Besprechungsräumen und die Organisation von Meetings gehören ebenso zu den Alltagsaufgaben des Direktionsbüros wie die laufende Dienstaufsicht über die Tätigkeiten der Arbeitsbereiche Baureferat, Zentrale Verrechnung, Expedit, Reinigungsdienst und Buchbinderei. Kernaufgaben sind der Ankauf und die Verwaltung der Sachmittel wie etwa Büro- und 
Verbrauchsmaterialien, Möbel und sonstige Einrichtungsgegenstände sowie die Erhebung des Investitionsbedarfs und die Durchführung von Investitionsprojekten für die gesamte Dienstleistungseinrichtung. Die Aufgabe des Budgetcontrollings zielt auf die gesamten Ausgaben der Dienstleistungseinrichtung und setzt bereits bei Ankaufsentscheidung ein. Für die Literaturmittel nimmt die Leiterin des Direktionsbüros zu diesem Zweck an den monatlichen Sitzungen der AG Budget teil. Sie sorgt für den reibungslosen Ablauf der Rechnungsfreigabe, der im Jahr 2016 auf ein elektronisches System umgestellt wurde. Ein Jahr zuvor wurden alle Bibliotheksschalter mit Bankomatkassen ausgestattet. Seither ist das Büro nicht nur für die Abrechnung von Handkassen und Barrefundierungen, sondern auch für die Bankomatkassenabrechnungen zuständig. Schon im Jahr 2013 wurde in der Hauptbibliothek ein Kassenautomat angeschafft, der ebenfalls vom Direktionsbüro abgerechnet wird. Auch Dienstreiseabrechnungen, das Ausstellen von Rechnungen für den Universitätslehrgang, für Kopierund Scanaufträge sowie die Administration von freien Dienstverträgen und Werkverträgen fallen in den Zuständigkeitsbereich des Direktionsbüros. Im Jahreslauf kommen weitere Agenden wie die Organisation des Betriebsausflugs und der Weihnachtsfeier hinzu. Das Büro ist somit ein zentrales Service im besten Sinn des Wortes.

\section{Zentrale Verrechnung}

Die Mitarbeiterinnen der Zentralen Verrechnung bearbeiten alle Rechnungen der Dienstleistungseinrichtung in SAP. Diese werden auf die buchhalterischen Merkmale überprüft und an die zuständigen Rechnungsprüfer_innen elektronisch weitergeleitet. Nach positiver Prüfung werden sie den Rechnungsfreigeber_innen weitergeleitet und anschließend in SAP gebucht sowie die Zahlung in der Dienstleistungseinrichtung Finanzwesen und Controlling in die Wege geleitet.

\section{Baureferat}

Das Baureferat ist für alle baulichen und einrichtungstechnischen Maßnahmen zuständig: Hier werden die Kosten für die aus den einzelnen Bereichen jährlich gemeldeten Investitionswünsche geschätzt und für die bewilligten Projekte jeweils mehrere Kostenvoranschläge eingeholt bzw. Ausschreibungen vorbereitet und mit der Dienstleistungseinrichtung Raum- und Ressourcenmanagement abgewickelt. Bei den Bau- und Einrichtungsprojekten ist das Baureferat für die ordnungsgemäße Abwicklung vor Ort und die Abnahme zuständig. Es gilt aber 
nicht nur, die regulär geplanten Investitionsprojekte im Jahresverlauf abzuarbeiten - es vergeht keine Arbeitswoche, in der es nicht auch Sofortmaßnahmen zu setzen gilt: Klemmende Türschlösser, verbogene Garderobenschlüssel, Schäden nach Wassereintritten, sich lösende Trittschutzkanten auf Treppen, tropfende Wasserhähne, kaputte Regalteile, durchgebrannte Leuchtmittel, zerbrochene Fensterscheiben, steckengebliebene Lifte, wackelnde Stuhlbeine und gebrochene Rückenlehnen gehören zum Arbeitsalltag.

\section{Expedit}

Das Team des Expedits ist für Transporte zwischen den über 40 Standorten der Dienstleistungseinrichtung, den Paket- und Briefversand zuständig. Täglich werden vormittags nach einem fixen Fahrplan bestimmte Fachbereichsbibliotheken und das Archiv sowie nachmittags das Depot der Hauptbibliothek in Simmering angefahren. Neben den regulären Fahrten zum Transport von Büchern und Büromaterialien gibt es regelmäßig auch Sondertransporte, etwa die Abholung von Buchschenkungen, die für die Schenkenden zum Transportieren zu groß, für eine Spedition aber zu klein sind; Besorgungen für Anlässe wie die »Nachtschicht@UB«; Transport von Equipment für Ausstellungen und Veranstaltungen; Abholung defekter IT-Geräte und Auslieferung reparierter oder neuer Computer, Bildschirme, Drucker, Handscanner usw. Die Fahrten erfolgen seit 2015 mit einem Elektroauto. Der Aufgabenbereich des Paket- und Briefversandes umfasst die zu versendenden Mahnschreiben für überfällige Buchentlehnungen, den Versand der via Fernleihe angefragten Bücher sowie die Retoursendungen von Fernleihebüchern anderer Bibliotheken und sonstige Printkorrespondenz.

\section{Kopierdienst}

Der Kopierdienst vervielfältigt Dokumente für interne Zwecke, Skripten für den Universitätslehrgang und auf Anfrage urheberrechtsfreie Werke für Benutzer_innen. Eine wichtige Tätigkeit des Kopierdienstes ist der Umgang mit Materialien am "Ende ihres Lebenszyklus«, d.h. das fachgerechte Entsorgen von Unterlagen mit personenbezogenen Daten: Alte Rechnungen, Schriftverkehr, aber auch die täglich anfallenden Bereitstellungsscheine nicht abgeholter Werke der Hauptbibliothek werden dem Schredder und direkt der zentralen Altpapierentsorgung zugeführt. Botengänge runden das Aufgabenprofil des Kopierdienstes ab. 


\section{Reinigungsdienst}

Der Reinigungsdienst ist für die Hauptbibliothek zuständig: Die Reinigung von Flächen im Größenausmaß der Hauptbibliothek (ca. $13.000 \mathrm{~m}^{2}$ ) erfordert gute Planung und stete Kontrolle. Die Koordination und Kooperation mit den externen Reinigungskräften sowie eine gleichzeitige Qualitätskontrolle liegt in den Händen einer internen Reinigungskraft. Eine jährliche Grundreinigung der Benutzungsbereiche sowie der internen Flächen, anlassbezogene Aufgaben, Entrümpelungsaktionen und Notfalleinsätze - wenn etwa im Benutzungsbereich Getränke verschüttet worden sind - gehören in das weite Aufgabenfeld des Reinigungsdienstes.

\section{Buchbinderei}

In der Buchbinderei werden beschädigte Bücher in einen Zustand gebracht, der eine hochfrequente Nutzung zulässt. Lose Blätter werden fixiert, Paperbacks bei Bedarf foliiert oder mit Hardcover versehen, Schutzumschläge eingeklebt.

Aufgrund der breiten Arbeitsfelder ist das Büro Bibliotheks- und Archivwesen universitätsintern und auch extern bestens vernetzt: Wichtige Partner sind insbesondere das Büro des Rektorats sowie die Dienstleistungseinrichtungen Finanzwesen und Controlling, Raum- und Ressourcenmanagement, Zentraler Informatikdienst, Personalwesen und Frauenförderung. Die Aufgabenvielfalt geht mit einer Vielzahl an Kooperationen und zwischenmenschlichen Kontakten einher, in deren Zentrum Maria Seissl in ihrer erfolgreichen und bewährten Art die Fäden zieht. 


\section{Vierzig Jahre Personal- und Statistikgeschichte des Bibliotheks- und Archivwesens der Universität Wien. Die Arbeit der Direktionsassistenz}

Anders als heute waren vor rund 40 Jahren sämtliche Personalangelegenheiten direkt in der Direktionsabteilung der Universitätsbibliothek Wien angesiedelt. Alle Anträge und sämtliche Änderungen wurden direkt an das jeweils zuständige Bundesministerium weitergeleitet, und auch die Antworten kamen von den Ministerien an die Direktionsabteilung der Universitätsbibliothek zurück. Mechanische Schreibmaschinen waren damals die einzige Schreibhilfe; alle Schriftstücke wurden zusätzlich mit Durchschlagpapier getippt.

Im Jahr 1977 bestand die Universitätsbibliothek Wien aus zehn Abteilungen in der Hauptbibliothek, aus drei Fakultätsbibliotheken, der Zentralbibliothek der physikalischen Institute und aus 20 Fachbibliotheken. Die Universitätsbibliothek hatte 173 Bedienstete, darunter 48 Personen, die im dezentralen Bereich arbeiteten. Durch die UOG-Novelle 1978 wurde der Bibliotheksdirektor/die Bibliotheksdirektorin verpflichtet, mit Ende jeden Kalenderjahres einen Bericht über das Bibliothekswesen der Universität an das oberste Kollegialorgan und an das Bundesministerium für Wissenschaft und Forschung zu erstatten. Dies war die Geburtsstunde des Statistikreferats und der Jahresberichte gem. $\$ 87$ Abs. 6 UOG.

In den folgenden Jahren wurden immer mehr Fachbibliotheken eingerichtet, die Fakultätsbibliothek für Medizin wurde gegründet und der Personalstand dementsprechend laufend erweitert. 1981 erfolgte ein Führungswechsel und Hofrat Ferdinand Baumgartner wurde zum Bibliotheksdirektor ernannt. Ab diesem Zeitpunkt brach das Computerzeitalter auch in der Universitätsbibliothek Wien an. Es begannen die Vorbereitungsarbeiten und die Organisationsplanungen für eine automatisierte Entlehnverbuchung. In dieser Zeit kam es auch zu den ersten Umbau-, Renovierungs- und Adaptierungsmaßnahmen in den schon in die Jahre gekommenen Räumlichkeiten.

Ab 1984 übernahm die Autorin dieses Beitrags offiziell die Leitung des Personalreferats mit einem Personalstand von 204 Bediensteten. Aufgrund des guten Einvernehmens mit dem zuständigen Bundesministerium war es der Universitätsbibliothek möglich, mehr Raum, Geld und Personal zu bekommen. 
Arbeitsplätze konnten höheren Entlohnungsgruppen zugeordnet und die Infrastruktur insgesamt verbessert werden. 1995 mussten für die Besoldungsreform der Beamtinnen und Beamten sämtliche Arbeitsplatzbeschreibungen auf siebenseitigen Formularen neu verfasst werden. Die Universitätsbibliothek Wien als größte wissenschaftliche Bibliothek Österreichs war dafür richtungsweisend. 1999 erfolgte die Besoldungsreform für Vertragsbedienstete, die jedoch nicht mehr so viel Aufwand bedeutete, da 1995 bereits für alle Mitarbeiter_innen Arbeitsplatzbeschreibungen verfasst worden waren.

Im Jahr 2000 erfolgte dann der nächste große Einschnitt: Die Fakultätsbibliothek für Medizin wurde eine eigene Dienststelle und infolgedessen ausgegliedert. Die Personalabteilung der Universität Wien übernahm die Durchführung der Personalangelegenheiten der Universitätsbibliothek Wien und damit auch teilweise das dafür zuständige Personal. Zu diesem Zeitpunkt gab es an der Universitätsbibliothek 245,5 Planstellen. Die größte Überraschung war allerdings eine neue ständige Stellvertreterin der damaligen Bibliotheksdirektorin, die die Versetzung von Tirol nach Wien wagte: Maria Seissl, die zuvor als Fachreferentin für Anglistik an der Universitätsbibliothek Innsbruck und Leiterin der Fachbibliothek für Germanistik der Universität Innsbruck tätig gewesen war.

Für die einheitliche Vorgangsweise bei der Ermittlung der Grundlagen für Auswertungen wurde die Arbeitsgruppe »Leistungsmessung in wissenschaftlichen Bibliotheken« vom Bundesministerium für Wissenschaft und Forschung eingerichtet. Damit einhergehend kam ein neuer Statistikbogen, der bis heute Verwendung findet.

Im Dezember 2003 wurde Maria Seissl zur provisorischen Leiterin des Bibliotheks- und Archivwesens. Damit brach eine neue Ära an.

In den Jahren 2004 und 2005 erhöhte sich der Personalstand erheblich, da das Personal des Archivs der Universität Wien und der Österreichischen Zentralbibliothek für Physik eingegliedert wurde und zusätzlich neue Stellen für geringfügig Beschäftigte geschaffen wurden. Somit waren mit 31.12.2005 281,3 Stellen zu betreuen. In diese Zeit fielen auch eine Analyse des Personaleinsatzes im gesamten Bereich der neu eingerichteten Dienstleistungseinrichtung (DLE) Bibliotheks- und Archivwesen und damit die Vorbereitung für die Einführung eines Kollektivvertrages. Die Verhandlungen des Dachverbandes der Universitäten und der Gewerkschaft dauerten insgesamt fünf Jahre. Der Kollektivvertrag wurde schließlich mit 1.10.2009 in Kraft gesetzt und für alle Mitarbeiter_innen, deren Beschäftigungsverhältnis nach dem 31.12.2003 startete, wirksam. Ebenfalls im Jahr 2005 bekam die DLE ihren ersten Lehrling und ist seit dieser Zeit Ausbildungsstelle für Lehrlinge des Lehrberufs »Archiv-, Bibliotheks- und InformationsassistentIn«. 
2006 kam es zur Einführung der elektronischen Zeiterfassung, die eine wesentliche Erleichterung bei der An- und Abwesenheitsverwaltung brachte. Bis dahin gab es ein Kardex-System. Jede Mitarbeiterin bzw. jeder Mitarbeiter, der/ die keine fixe Arbeitszeit hatte, musste sich täglich beim Kommen zum und Verlassen des Arbeitsplatzes je nach Ort desselben entweder im »Direktionsgang» oder im "Unteren Kanzleigang» (= heutiger Gang vor der Abteilung Formalerschließung) schriftlich ein- und austragen. Zur leichteren Abrechnung am Monatsende wurde in Viertelstundenschritten gerechnet - heute wird bereits minutenweise abgerechnet. Für das Statistikreferat bedeutete dieses Jahr auch die erstmalige Teilnahme an der Erhebung von Daten und Indikatoren für den Bibliotheksindex BIX sowie an der Wissensbilanz der Universität Wien.

Ende 2008 startete die Bibliotheksdirektion den Strategieentwicklungsprozess »Vision 2020«. Im Zuge dessen fanden drei Großgruppentage statt, an denen alle Mitarbeiter_innen teilnehmen konnten und die zunächst von einer Steuerungsgruppe ein Jahr lang vorbereitet wurden. Mit dem dritten Großgruppentag im Jahr 2011 wurde der Strategieentwicklungsprozess zwar formal abgeschlossen, aber nicht beendet, da die »Vision 2020« weiterhin gilt und Ausgangspunkt für die ständige Weiterentwicklung der Strategie ist.

Im Jahr 2010 wurde die DLE Bibliotheks- und Archivwesen erstmalig der turnusmäßigen Peer-Evaluation unterzogen und 2014 begann die Evaluierung der bibliothekarischen Kernprozesse. 2015 wurde der elektronische Urlaubsschein eingeführt und im Jahr darauf von der Personalabteilung der Universität Wien das elektronische Urlaubsblatt für alle Mitarbeiter_innen verpflichtend. 2018 gab es wieder eine turnusmäßige Evaluierung durch Expertinnen und Experten aus dem internationalen wissenschaftlichen Bibliothekswesen.

Anhand der vielen Änderungen und Entwicklungen, die die Abteilung in den letzten Jahrzehnten erlebt hat, lässt sich die dynamische Weiterentwicklung der Universitätsbibliothek, aber auch der Universität Wien im Allgemeinen sehr gut ablesen. Wir sehen unsere Aufgaben letztlich auch genau darin, diesen ständigen Prozess zu begleiten, mitzugestalten und an die Mitarbeiterinnen und Mitarbeiter zu kommunizieren. Die Dienstleistungseinrichtung Bibliotheks- und Archivwesen ist damit auch für künftige Veränderungen gut vorbereitet und gerüstet. 
Open-Access-Publikation im Sinne der CC-Lizenz BY-NC-ND 4.0

(c) 2019, Vandenhoeck \& Ruprecht GmbH \& Co. KG, Göttingen ISBN Print: 9783847110989 - ISBN E-Lib: 9783737010986 


\section{Man kann nicht nicht kommunizieren ... Team Öffentlichkeitsarbeit}

Öffentlichkeitsarbeit und Marketing zählen heute zu den Kernaufgaben von Bibliotheken und werden im Normalfall bereits bei der strategischen Planung neuer Services mitgedacht. Das ist gewiss nicht immer so gewesen. Auch wenn Bibliotheken seit jeher als Auskunftgeber für Benutzer_innen agieren, war dies lange Zeit kein bewusster Akt. Zumindest wäre kaum jemand auf die Idee gekommen, dass dies einen Auftritt nach außen darstellt, der elementar für das Image der Bibliothek ist. ${ }^{2}$

\section{Vom »Team Veranstaltungen und Ausstellungen« zum zentralen Service »Öffentlichkeitsarbeit«}

Die Hauptbibliothek hatte während der 1990er und bis Mitte der 2000er Jahre in der Hauptabteilung Benutzung mit Sieghard Neffe einen sehr engagierten Leiter, der den Fokus auf die Universitätsbibliothek Wien als Kulturinstitution legte. Das hatte mehrere Gründe, allen voran die rechtliche Situation der Universitätsbibliothek als eigenständige Einrichtung, die nicht der Universitätsleitung, sondern dem damaligen Wissenschaftsministerium unterstellt war. Mit der Eingliederung in die Universität Wien und der Schaffung der Dienstleistungseinrichtung Bibliotheks- und Archivwesen im Jahr 2004 musste sich mittelfristig auch das Aufgabenportfolio des Teams "Veranstaltungen und Ausstellungen" ändern. Der Fokus lag zu dieser Zeit auf Veranstaltungen und Ausstellungen, für die das damalige Team der Fachreferent_innen auch inhaltlich verantwortlich zeichnete. Was aus dieser Zeit nicht nur beibehalten, sondern intensiviert wurde,

1 Paul Watzlawick, Janet H. Beavin, Don D. Jackson: Menschliche Kommunikation. Formen, Störungen, Paradoxien. 12., unveränd. Aufl. Bern: Huber 2011, S. 60.

2 Zum Verständnis von Öffentlichkeitsarbeit in Bibliotheken vgl. Marion Schmidt: Auf dem Weg zur bibliotheksspezifischen Öffentlichkeitsarbeit. Wiesbaden: Harrassowitz 2001 (Beiträge zum Buch- und Bibliothekswesen 41) bzw. Ursula Georgy, Frauke Schade (Hg.): Handbuch Bibliotheks- und Informationsmarketing. Berlin: De Gruyter 2012. 
sind sowohl inneruniversitäre als auch externe Kooperationen. So wurden zum Beispiel 2005 gemeinsam mit dem Alumniverband die "Bibliothekslesungen" gegründet, die bis heute mehrmals jährlich unter großem Publikumsinteresse stattfinden.

Dennoch war bereits zu diesem Zeitpunkt absehbar, dass sich für die Zukunft der Schwerpunkt weg von Eventmanagement hin zu Öffentlichkeitsarbeit verlagern würde müssen. Dementsprechend wurde Ende 2007 eine Abteilung für Öffentlichkeitsarbeit eingerichtet, die nicht mehr nur für die Hauptbibliothek zuständig war, sondern als Serviceeinrichtung für die gesamte Universitätsbibliothek fungieren sollte. Kernaufgabe der neu gegründeten Abteilung war die Schaffung einer Corporate Identity für die Dienstleistungseinrichtung Bibliotheks- und Archivwesen, primär eines Corporate Designs, das den gemeinsamen Außenauftritt aller Teilbibliotheken sicherstellen würde. Das war wegen der in den Jahren zuvor erfolgten Eingliederung zahlreicher früherer Instituts- und Fakultätsbibliotheken in die Dienstleistungseinrichtung dringend notwendig allein aufgrund der umfänglichen neuen Bezeichnung neben dem eingeführten Namen »Universitätsbibliothek Wien« bzw. »UB Wien« allerdings kein Selbstläufer. Herausfordernd wurde der durch die engere Zusammenarbeit mit der Dienstleistungseinrichtung Öffentlichkeitsarbeit der Universität notwendige Umstieg auf ein neues Grafikprogramm, der einerseits einen großen Schulungsaufwand erforderte, andererseits die Neugestaltung der Drucksorten erleichterte. Die Servicierung der Fachbereichsbibliotheken zählt bis heute zu den ressourcenaufwändigsten Aufgaben der Abteilung und umfasst Imagefolder, Leitsystem, Informationsmaterialien, aber nach wie vor auch Drucksorten für Veranstaltungen, wobei sich das Portfolio mittlerweile auch um Kongressmaterialien und Werbemittel erweitert hat. Zur Verbesserung der internen Kommunikation wurde im Herbst 2008 ein interner Newsletter gegründet, der bis heute alle zwei Monate erscheint und dessen Redaktion derzeit zwei Mitglieder des Teams Öffentlichkeitsarbeit angehören, wobei diesem auch die grafische Gestaltung obliegt.

An der Universität Wien wurde 2017 ein neues Corporate Design implementiert, mit dem unter anderem die Dachmarke Universität gestärkt werden sollte, was dazu führte, dass die Universitätsbibliothek zum vierten Mal innerhalb von 15 Jahren ihr Logo - jetzt nur mehr eine »Wort-Bild-Marke« - änderte. Dies machte einen eigenen Markenentwicklungsprozess zur Stärkung des Außenauftrittes der Dienstleistungseinrichtung notwendig, der Ende 2018 abgeschlossen wurde. Erstmals lag der Fokus nicht auf den Drucksorten, sondern es wurde von Beginn an der Webauftritt mitgedacht. In den Prozess eingebunden waren neben dem Rektorat und der Dienstleistungseinrichtung Öffentlichkeitsarbeit auch zahlreiche Kolleg_innen der Dienstleistungseinrichtung Bibliotheks- und Archivwesen. Eine spezielle Verwendung von Typographie steht 
der Dienstleistungseinrichtung nun als Alleinstellungsmerkmal zur Verfügung, was auch in das Corporate-Design-Manual der Universität Eingang gefunden hat.

\section{Von Prototypen und Zielgruppen}

Der partizipativ angelegte Strategieentwicklungsprozess 2009/2010 führte zu einer Reihe von "Prototypen«, die in vielen Bereichen Auswirkungen auf die interne und externe Kommunikation der Dienstleistungseinrichtung hatten. Ein großes Thema ist bis heute das interne Wissensmanagement, was sowohl im neu implementierten Intranet der Universität Niederschlag fand, als auch die Aktualisierung des Intranets der Dienstleistungseinrichtung zur Folge hatte. Für neue Mitarbeiter_innen wurde in diesem Zusammenhang ein »Welcome Kit» erstellt, das mittlerweile ebenfalls im Intranet der Dienstleistungseinrichtung zu finden ist. Die Ideendatenbank, ein niedrigschwelliges Tool für internes Vorschlagswesen, ist ein weiterer Prototyp, der seit einigen Jahren an die Öffentlichkeitsarbeit angedockt ist, da viele Vorschläge in den Bereich Produktmarketing fallen.

Die Dienstleistungseinrichtung hat seit 2005 umfangreiche Services für Forschende entwickelt, die permanent erweitert und verbessert werden. $\mathrm{Zu}$ den Aufgaben des Teams Öffentlichkeitsarbeit gehört die Sichtbarmachung dieser Services innerhalb der Universität, wobei sich die Erreichbarkeit dieser Zielgruppe als besonders herausfordernd darstellt. Die neue Grafik, die sowohl im Web als auch in Drucksorten ihren Niederschlag findet, ist Teil eines Konzeptes, das auch ein aktives Zugehen auf die Forscher_innen beinhaltet und dessen Umsetzung eine zentrale Aufgabe der kommenden Jahre sein wird.

Für die größte Zielgruppe - jene der Studierenden - wurde das bis heute erfolgreiche Format »Nachtschicht@UB« eingerichtet, dessen Organisation im Regelbetrieb in die Abteilung Öffentlichkeitsarbeit überging. Dafür erhielt das Nachtschicht-Team 2013 den Jahrespreis der Universität. Die Universität Wien hat in dieser Zeit neue Formate für Studienanfänger_innen entwickelt, z. B. die »UniLeben « und die »Uniorientiert», an denen die Universitätsbibliothek mit Messeständen, Führungen sowie Vorträgen beteiligt ist. Speziell die Studierenden nutzen den etwas weniger formellen Kommunikationskanal der sozialen Medien, wobei die Dienstleistungseinrichtung aktuell Facebook und Twitter bedient. 


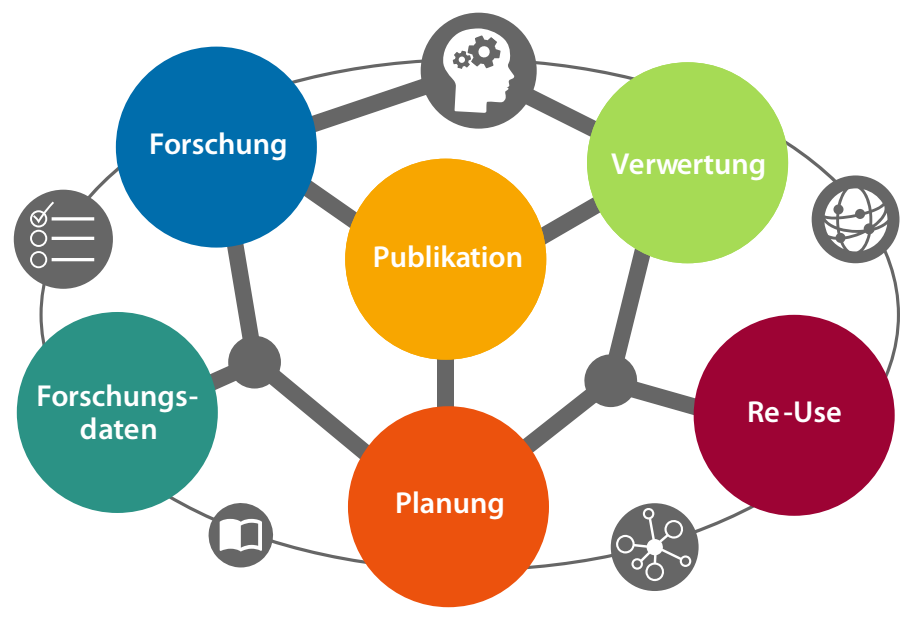

\section{Und doch wieder Veranstaltungen ...}

Die baulichen Veränderungen in der Hauptbibliothek reduzierten die Ausstellungsflächen massiv, sodass die noch vorhandenen Vitrinen nun der Präsentation von Bibliotheksservices und den Neuerscheinungen der »Vienna University Press« dienen. Kleinere Veranstaltungen werden an die Fachbereichsbibliotheken verlegt, so zum Beispiel die Alumnilesungen an die Germanistik oder die Kriminacht an die Geschichte. Einige wenige, wichtige und große Veranstaltungen wie der Launch der European Science Cloud im Herbst 2018 wurden im Großen Lesesaal der Hauptbibliothek durchgeführt, gelegentlich darf hier auch getanzt werden - allerdings nur vom Staatsopernballett für das Neujahrskonzert oder bei der internen Weihnachtsfeier.

Seit 2012 hat die Abteilung mit der Durchführung mehrerer Konferenzen ihre Expertise erweitert, unter anderem der Liber 2012, des Österreichischen Bibliothekartags 2015 mit mehr als 1.000 Teilnehmer_innen sowie der InetbibOdok 2018.

Besonders intensiv war das Jahr 2015 mit dem 650-Jahr-Jubiläum der Universität Wien, an dem die Dienstleistungseinrichtung Bibliotheks- und Archivwesen mit der Webseite zur Geschichte der Universität Wien, der Beteiligung an Publikationen, mehreren Ausstellungen sowie Veranstaltungen, aber auch einer Bibliotheksralley und der Entwicklung eines Bibliotheksquartetts beteiligt war. 2018 war der Campus der Universität durch das 20-Jahr-Jubiläum im Fokus der Veranstaltungen, woraus sich das neue Format "Erlesenes Erforschen« entwickelt hat, bei dem Forscher_innen der Universität Wien ihre Publikationen vorstellen. 


\section{Reiseziele}

Für die Zukunft ist zu erwarten, dass das Team Öffentlichkeitsarbeit weiterhin als wichtige Schnittstelle zu anderen Einrichtungen der Universität und auch außerhalb fungieren wird.

Synchrone Kommunikationsformen wie Chat, Instant Messaging oder auch Chatbots werden noch mehr Raum einnehmen als die asynchronen wie E-Mail oder Webformulare. Auch bei der Kommunikation wird, wie bei vielen anderen Bibliotheksservices, erwartet, dass jederzeit Ansprechpartner_innen zur Verfügung stehen. Klassische Medienarbeit wird nach wie vor benötigt, aber gleichzeitig müssen auch die Kommunikationskanäle im Internet, in der die Reaktionen wesentlich schneller kommen müssen, mit Informationen versorgt werden. Welche Kanäle für die Bibliothek in Zukunft relevant sein werden, lässt sich derzeit noch nicht absehen. Weiterbildung wird es jedenfalls in Bereichen wie Video oder Podcast brauchen, um auch in Zukunft unsere Zielgruppen erreichen zu können. Vielleicht werden die zukünftigen Tutorials der Bibliothek auf Youtube abrufbar sein oder Forscher_innen informieren sich auf Videokonferenzen über die forschungsunterstützenden Services, während wir unsere Services gemeinsam mit den Nutzer_innen in Weltcafés weiterentwickeln. 
Open-Access-Publikation im Sinne der CC-Lizenz BY-NC-ND 4.0

(c) 2019, Vandenhoeck \& Ruprecht GmbH \& Co. KG, Göttingen ISBN Print: 9783847110989 - ISBN E-Lib: 9783737010986 


\section{Lifelong Learning for Librarians. Das Angebot des Teams Aus- und Fortbildung}

Die Anforderungen im Berufsleben steigen stetig und somit ist es heute wichtiger denn je, sich fortzubilden. Mittlerweile steht an der Universitätsbibliothek Wien ein reichhaltiges Repertoire an Kursen und Lehrgängen zur Auswahl bereit. Das Team Aus- und Fortbildung kümmert sich um den reibungslosen Betrieb und koordiniert die komplexen Abläufe, welche Aufgaben wie Durchführung von Lehrgängen, Betreuung von Fortbildungsangeboten, aber auch Entwicklung neuer Formate mit sich bringen.

\section{Universitätslehrgang}

Aufgrund des Inkrafttretens des Universitätsgesetzes 2002, welches eine einheitliche Ausbildung für das Bibliothekspersonal vorgeschrieben hat, wurde 2004 der Universitätslehrgang Library and Information Studies MSc eingerichtet. Dieser ist zweistufig, bestehend aus Grund- und Masterlehrgang, und wird aktuell an der Universitätsbibliothek Wien in Kooperation mit der Österreichischen Nationalbibliothek und den Universitäten Innsbruck und Graz durchgeführt. Die Studierenden des Universitätslehrgangs erwerben neben Kenntnissen im Bereich des Bibliothekswesens auch praxisorientierte Berufskompetenzen. Etliche Mitarbeiter_innen verschiedener Bereiche und Fachbereichsbibliotheken der Universitätsbibliothek haben diese Ausbildung abgeschlossen und viele von ihnen sind heute in führenden Positionen tätig.

Die Bezeichnung »Akademischer Bibliotheks- und Informationsexperte/ -expertin«steht erfolgreichen Absolvent_innen des Grundlehrganges zu, der akademische Grad »Master of Science (MSc) Library and Information Studies" wird Absolvent_innen des Masterlehrgangs verliehen. Seit 2004 ist Maria Seissl für die organisatorische Lehrgangsleitung zuständig. Sie entscheidet in allen Angelegenheiten des Universitätslehrgangs und wird vom Team Aus-und Fortbildung dabei unterstützt. 
Um unsere Teilnehmer_innen in jedem Lehrgang aufs Neue auf die sich stets ändernden Anforderungen in ihrem Arbeitsumfeld vorzubereiten, werden Entwicklungen in diesem Berufsfeld beobachtet und in die Erstellung der Curricula eingearbeitet. Dazu ist es unumgänglich, mit den Verantwortlichen der oben genannten Partnerinstitutionen und dem Bundesministerium für Bildung, Wissenschaft und Forschung fachlich intensiv und professionell zusammenzuarbeiten, was in unserem Falle reibungslos funktioniert. Über das Berufliche hinaus prägen auch persönliche Wertschätzung und Freundschaften die $\mathrm{Zu}$ sammenarbeit. Die Arbeitsgemeinschaft der Ausbildungsverantwortlichen Österreichs für das Bibliothekspersonal der Universitäten, kurz ARGE Ausbildung, tagt mehrmals jährlich und erarbeitet Curricula für Lehrgänge, aber auch bedarfsorientierte Ausbildungsangebote auf universitärem Niveau.

Als Innovation ab 2019 bieten wir die zusätzliche Möglichkeit an, den Grundlehrgang nunmehr auch berufsbegleitend über die Dauer von drei Semestern zu absolvieren, um auch berufstätigen Bewerber_innen den Zugang zum Bibliothekswesen zu öffnen.

\section{Zertifikatskurse}

Liegt die Absolvierung des Grundlehrgangs schon einige Jahre zurück oder möchte jemand seine Kenntnisse bezüglich aktuellster Entwicklungen im Bibliothekswesen auffrischen, bieten wir seit 2015 Zertifikatskurse mit jeweils drei Modulen als Weiterbildungsformat an. Je nach Tätigkeitsbereich entscheiden sich die Teilnehmer_innen für einen der drei Kurse "Bibliotheken führen", »Bestandsmanagement in Bibliotheken und Informationseinrichtungen« oder »Data librarian«. Das besondere Merkmal im Ablauf dieser Kurse ist der dabei entstehende spannende Austausch der Teilnehmer_innen durch die Aufteilung der drei einzelnen Module auf die Partner-Universitätsbibliotheken Wien, Graz und Innsbruck.

\section{Ausbildungsangebote für Lehrlinge und Mitarbeiter_innen mit abgeschlossener Schulpflicht}

Auch 2004 ins Leben gerufen wurde die dreijährige Lehrlingsausbildung zur/ zum »Archiv-, Bibliotheks- und InformationsassistentIn«, welche sich großer Beliebtheit erfreut. Der Unterricht findet sowohl in der Berufsschule für Handel und Reisen in Wien als auch im Lehrbetrieb an der Universitätsbibliothek statt, 
wo den Lehrlingen beste Grundlagen für ihr zukünftiges Berufsleben mitgegeben werden.

Unser großes Ausbildungsangebot beinhaltet auch den Lehrgang »Bibliothek, Information und Dokumentation«. Dieser richtet sich an Mitarbeiter_innen mit Pflichtschulabschluss in wissenschaftlichen Bibliotheken. Mit dem erfolgreichen Abschluss dieses Lehrgangs erwerben die Teilnehmer_innen ein Universitätszertifikat.

\section{Externe und interne Fortbildung}

Im Rahmen des Erasmus-Programmes für das Universitätspersonal dürfen wir an der Universitätsbibliothek Wien auch immer wieder Bibliothekar_innen aus verschiedenen europäischen Ländern willkommen heißen. Jedes Jahr haben wir bis zu zehn Kolleg_innen in unserem Haus, mit denen wir uns rege über die jeweiligen Arbeitsabläufe austauschen. Im Gegenzug besuchen Mitarbeiter_innen aus unserer Bibliothek im Rahmen dieses Austauschprogrammes andere Bibliotheken, was für alle Beteiligten einen großen Erfahrungszugewinn bedeutet.

Besonders hervorzuheben ist das neue Seminarprogramm zur internen Fortbildung an der Universitätsbibliothek Wien, welches im Sommersemester 2019 startete und ebenfalls von Maria Seissl geleitet wird. Es heißt »4L - Lifelong Learning for Librarians « und wird österreichweit angeboten. Die Kurse finden in Wien, Graz und Innsbruck statt. Das Programm umfasst Themenbereiche wie elektronische Ressourcen, Recht und Datenschutz, Repositorien, Erschließung und speziell auf Anfragen angepasste Kurse, die für alle Bibliotheksmitarbeiter_innen von großer Relevanz sind und eine praxisorientierte und spezialisierte Fortbildung garantieren. 
Open-Access-Publikation im Sinne der CC-Lizenz BY-NC-ND 4.0

(c) 2019, Vandenhoeck \& Ruprecht GmbH \& Co. KG, Göttingen ISBN Print: 9783847110989 - ISBN E-Lib: 9783737010986 


\section{Innovation zwischen Vision und Realität. Die Stabstelle ubw:innovation an der Universitätsbibliothek Wien}

\section{Hintergründe}

Infolge der Umbrüche im österreichischen Universitätswesen zu Beginn des 21. Jahrhunderts, die u. a. die Eingliederung der bis dahin selbstständigen Universitätsbibliotheken in die Verwaltung und Strukturen der Universitäten mit sich brachten, zeigte sich bald, dass eine Neuausrichtung in den Zielsetzungen von akademischen Bibliotheken notwendig wurde. Darüber hinaus erlebten Bibliotheken die ersten radikalen Auswirkungen der "Digitalen Revolution" durch zunehmende Durchdringung des Bibliotheksalltags mit digitalen Services und einer ständig steigenden Konkurrenz in der Domäne der Informationsgewinnung durch Suchmaschinen.

An der Universitätsbibliothek Wien wurde diese notwendige Neupositionierung mit dem »Strategieentwicklungs-Prozess« im Jahr 2009 begonnen. Unter Beteiligung vieler Kolleg_innen entstanden zahlreiche innovative Ansätze zur Neugestaltung der Bibliothek. Die wichtigsten Ergebnisse wurden in einem umfangreichen internen Visions-Papier sowie einer veröffentlichten Strategie, die vorerst bis zum Jahr 2015 ausgerichtet war, zusammengefasst. Um dem Prozess der innovativen Ideenfindung und Implementation neuer Services Nachhaltigkeit zu verleihen, wurde eine Stabstelle für Innovationsmanagement unter der Bezeichnung "ubw:innovation « etabliert. ${ }^{1}$

Impulsgebend war die zu Beginn des 21. Jahrhunderts auch im deutschen Sprachraum geführte Diskussion über die Einführung von Innovationsmanagement als Werkzeug zur strategischen Betriebsführung an akademischen und öffentlichen Bibliotheken. Seit der ersten Hälfte des 20. Jahrhunderts als Prozess der Durchsetzung von technischen oder organisatorischen Neuerungen in

1 Zur Etablierung und zu ersten Erfahrungen von ubw:innovation siehe: Maria Seissl, Wolfram Seidler: Strategieentwicklung und Innovation an der Universitätsbibliothek Wien. In: Andreas Degkwitz (Hg.): Personal- und Organisationsentwicklung in Bibliotheken. Berlin: De Gruyter 2013, S. 197-202. 
Wirtschaftsbetrieben bekannt, wurden, ausgehend von den USA, die Methoden des Innovationsmanagements zu Ende dieses Jahrhunderts auch auf das Management öffentlicher Organisationen angewandt, mit etwas Verzögerung schließlich auch an deutschsprachigen Bibliotheken. Die Vorreiterrolle übernahm hier die Bibliothek der ETH Zürich.

Geht es bei Innovationsmanagement in der Privatwirtschaft in erster Linie um die Generierung und Einführung radikal neuer Produkte und Dienstleistung in einem Markt sich gegenseitig konkurrierender Unternehmen, bedeutet es im Bereich öffentlicher Bibliotheken meist die Verbesserung und Modernisierung vorhandener Dienstleistungen sowie die Adaption neuer Technologien. ${ }^{2}$ Die Dienstleistung der Informationsvermittlung an sich muss nicht neu erfunden werden, es muss jedoch den sich ändernden gesellschaftlichen, organisatorischen und technologischen Rahmenbedingungen durch Neugestaltung der Services Rechnung getragen werden.

\section{Die Innovationsstelle der Universitätsbibliothek Wien}

Die Aufgaben der Innovationsstelle an der Universitätsbibliothek Wien waren zunächst sehr weit gefasst und sollten in erster Linie dem Aufbau eines innovationsfreundlichen Umfeldes innerhalb der Bibliothek dienen. Als weitere Kernaufgaben der neuen Stabstelle wurde die Beobachtung und Analyse von Trends, die Durchführung von Projektberatung und -entwicklung sowie die Unterstützung der Bibliotheksleitung durch Ausarbeitung von strategischen Überlegungen festgelegt. Zur Sammlung der Vorschläge wurde eine Ideendatenbank eingerichtet, die anfangs sehr rasch befüllt wurde. Durch die Schaffung von zeitlichen und personellen Freiräumen sollten innovative Ideen der Mitarbeiter_innen ohne Erfolgsdruck als Prototypen auf deren Realisierbarkeit geprüft und im Idealfall etabliert werden. Als Innovationen wurden »Dienstleistungen, die im österreichischen Bibliothekswesen noch nicht eingesetzt werden oder nur wenig verbreitet sind ${ }^{3}$ definiert. Tatsächlich gelang es der Universitätsbibliothek Wien in den folgenden Jahren, eine Vorreiterrolle beim Aufbau neuer Services - insbesondere im Bereich Forschungsunterstützung - innerhalb des österreichischen Bibliothekswesens einzunehmen.

Lediglich das Konzept der innovationsfördernden Freiräume erwies sich als an der Universitätsbibliothek Wien nicht anwendbar. Die Methode der Proto-

2 Siehe dazu Rudolf Mumenthaler: Innovation nicht nur in großen Bibliotheken. In: Bibliotheksdienst 48 (2014), Nr. 5, S. 345-349. DOI: 10.1515/bd-2014-0045.

3 Interner Newsletter der Dienstleistungseinrichtung Bibliotheks- und Archivwesen der Universität Wien, Nr. 12, 15.07.2010, S. 4. 
typen verursachte zahlreiche Reibungspunkte im internen Getriebe. Viele Aktivitäten fanden quer zur Linienorganisation statt, was zu Problemen mit Verantwortlichkeiten führte. ${ }^{4}$ Was als »Spielwiese« initiiert war, wurde im Lauf der Zeit als "Zeitverschwendung" gesehen, Projekte, Prototypen und Vorhaben scheiterten an ökonomischen Zwängen. Es folgte die ernüchternde Erkenntnis, dass im Unterschied zu anderen Bibliotheken, die sich international im Gebiet der Umsetzung innovativer Ideen einen Namen machten, die Ausstattung mit den notwendigen personellen und finanziellen Ressourcen an der Universitätsbibliothek Wien nicht gegeben war.

\section{Neuansätze}

Veränderungsprozesse verlaufen selten linear zielführend. Die Erfahrung, dass anfänglicher Euphorie meist Desillusionierung folgt, ist universell und nicht nur auf das Berufsleben beschränkt. Doch ebenso generell setzt meist anschließend ein Lernprozess ein, der ermöglicht, die gewonnenen Erkenntnisse in eine produktive Phase überzuleiten.

2012 erfolgte zeitgleich mit der personellen Umbesetzung, bei der die Autorin dieses Beitrags mit Wolfram Seidler die Leitung des Teams Bibliotheksysteme mit der Leitung von ubw:innovation abtauschte, eine Neuausrichtung der Agenden der Stabstelle. Hauptaugenmerk wird seither auf die drei Bereiche Strategieverfolgung und -entwicklung, Trendbeobachtung und Projektcontrolling gelegt. Mit der Ausarbeitung eines strategischen Konzepts zur Organisationsentwicklung im Jahr 2010 hat sich die Universitätsbibliothek Wien ein großes Portfolio zur Umsetzung auferlegt. Dass dies auch tatsächlich geschieht und der Fokus auf notwendige Neuerungen nicht im Alltagsgeschäft verloren geht, ist Aufgabe der systematischen Strategieverfolgung, ebenso wie die mögliche Adaptierung der Ziele in einer sich rasch ändernden Umwelt. Die Beobachtung von technischen und organisatorischen Trends in der internationalen Bibliothekswelt fließt in die Ausarbeitung von strategischen Überlegungen und Konzepten ein, die die Bibliotheksleitung bei ihren Entscheidungsfindungen unterstützen. Der dritte Schwerpunkt Projektcontrolling unterstützt bei der Konzeption und der Durchführung von bibliotheksinternen Projekten und Arbeitsgruppen.

Diese Neuorientierung brachte mit sich, dass es um »die Innovation « in der Bibliothek leiser wurde. Das spektakuläre Erproben innovativer Ideen wurde zugunsten der Umsetzung ausgewählter Anliegen in Arbeitsgruppen und Projekten modifiziert. Am Anfang standen der Abschluss offener Arbeitseinheiten, die Dokumentation aller Projekte und Arbeitsgruppen im Intranet sowie die

4 Siehe Seissl/Seidler, S. 201. 
Erstellung eines internen Leitfadens für Projektmanagement. Im Rahmen des »Projekttags 2013« wurden alle laufenden Projekte und Arbeitsgruppen intern den Mitarbeiter_innen der Bibliothek präsentiert (der Projekttag wurde wegen großen Erfolgs 2017 wiederholt). Der Verbesserung der internen Kommunikation diente auch die Neugestaltung des Intranets der Bibliothek. Für die Kommunikation nach außen wurde unter dem Schlagwort »Open Innovation « ein "Ideenforum« auf den Webseiten der Bibliothek eingerichtet. Als weitere Meilensteine können die Ausarbeitung eines Konzepts für Sucheinstiege, von Richtlinien und einer Policy für die Lizenzvergabe an von der Bibliothek verwalteten digitalen Objekten, die Einführung der Vergabe von DOI und die Adaptierung der Strategie bis zum Jahr 2020 genannt werden.

Die Handlungsfelder der unmittelbaren Zukunft stehen weiterhin im Zeichen der Strategieverfolgung. Einerseits sind noch offene strategische Ziele bis 2020 umzusetzen, wie etwa die Ausarbeitung und Einführung von Marketingmaßnahmen für die forschungsunterstützenden Services, andererseits steht die Neukonzeption einer Bibliotheksstrategie für die Jahre nach 2020 an. 


\section{Die Webredaktion im Spannungsfeld}

Websites haben sich im Laufe der vergangenen zwei Jahrzehnte zu wesentlichen Kommunikations- und Informationsmedien entwickelt, die die Art und Weise, wie Informationssuchende Antworten auf ihre Fragen finden, grundlegend verändert haben: Zuvor war ein direkter Kontakt zwischen Fragenden und Antwortenden nötig, im Zuge dessen meist persönlich konkrete Fragen gestellt wurden, die dann direkt beantwortet wurden. Dies bedingte eine gewisse räumliche Nähe; die Kommunikation blieb meist in lokalem Rahmen.

Mit dem World Wide Web ergab sich die Möglichkeit, diese lokale Barriere zu überwinden und weltweit Zugriff zu Informationen zu erlangen. Die Herangehensweise von Informationssuchenden änderte sich jedoch grundlegend, da im Gegensatz zum persönlichen Kontakt mit Frage und Antwort das Prinzip der Website darin besteht, dass Informationen dort lediglich bereitgestellt werden und die Informationssuchenden in den bereitgestellten Daten nach der Antwort suchen müssen, zwar in der Annahme, dass sich die Information dort befindet, aber ohne Gewissheit, dass das wirklich der Fall ist.

Es ist klar, dass diese Art der Informationssuche deutlich fehleranfälliger sein kann. Es ergeben sich Szenarien, die beim persönlichen Kontakt nicht auftreten können: So kann einerseits die Information ganz einfach nicht vorhanden sein, weil die Informationsanbietenden sie nicht für relevant hielten oder die entsprechende Frage nicht erwartet haben. Andererseits kann die Information zwar vorhanden sein, aber von den Informationssuchenden nicht gefunden werden, oder sie wird zwar gefunden, aber nicht oder falsch verstanden.

Jede dieser vier Möglichkeiten tritt umso öfter auf, je größer die Organisation ist, die die Website betreibt, und je größer, umfangreicher und komplexer das Ausmaß der auf der Website bereitgestellten Daten ist.

Dies bedingt, dass ab einer gewissen Organisations- und somit WebsiteGröße eine Webredaktion unabdingbar ist. Ihre Aufgabe ist, sicherzustellen, dass die Website alle relevanten Informationen enthält und diese regelmäßig aktualisiert werden; dass fehlende Informationen möglichst zeitnah ergänzt werden; dass die Website so übersichtlich strukturiert ist, dass Informationen gut ge- 
funden werden; und dass Texte so klar formuliert sind, dass die Informationen auch ohne Fachwissen verstanden werden.

Diese Aufgaben können nur erfüllt werden, wenn die Webredaktion weiß, wonach die Benutzenden suchen, um Entscheidungen über die Relevanz treffen zu können, welche Informationen nicht gefunden wurden, um diese ergänzen zu können, wie die Benutzenden nach Informationen suchen, um entsprechende Strukturen bauen zu können, und welche Begriffe allgemein verwendet werden, um das Wording entsprechend anzupassen.

Wesentlich mehr als beim persönlichen Kontakt ist es somit notwendig, dass die Informationsanbietenden die Bedürfnisse der Benutzenden kennen und sich so weit in sie hineinversetzen können, dass diese antizipiert und entsprechend bedient werden können.

Benötigt ein Webmaster oder eine Webmasterin in erster Linie technische Fertigkeiten, so benötigt ein Mitglied einer Webredaktion vor allem Einfühlungsvermögen und kommunikative Fertigkeiten. Eine gute Website ist stets für die Benutzenden gebaut und nicht für die Organisation, die sie betreibt.

Hier breiten sich die Spannungsfelder aus, in denen sich Mitglieder von Webredaktionen bewegen, und deren Ursache darin liegt, dass sie von der Organisation angestellt sind, die die Informationen bereitstellt, aber eigentlich für die Benutzenden arbeiten sollten, die die Informationen suchen.

\section{Spannungsfeld 1: Informationsbedürfnisse der Benutzenden vs. Marketingbedürfnisse der Organisation}

War in den Anfängen des World Wide Web die Mehrzahl der Websites noch informationsorientiert, so dienen heute im hochkommerzialisierten Internet die meisten Websites vor allem Marketingzwecken und der Selbstdarstellung von Firmen und deren Produkten. Der Informationscharakter ist dabei oft auf die verkaufsfördernden Inhalte beschränkt.

Dieses Marktdenken hat sich im Laufe der Zeit auch in den Bildungsbereich ausgedehnt, weil auch dort, begünstigt durch stetigen finanziellen Druck, die Organisationen gezwungen sind, vermehrt den Fokus auf Selbstdarstellung und die Präsentation erbrachter Leistungen zu legen. Dabei sind, wie jüngste Usability-Studien festgestellt haben, einzelne Websites von Bildungseinrichtungen inzwischen von Informationsangeboten zu Marketinginstrumenten "gekippt" und können somit ihre ursprüngliche Funktion als Informationsquelle für die Benutzenden nur noch unzureichend erfüllen. 
Aufgabe der Webredaktion ist es, in diesem Spannungsfeld zu vermitteln und besonders darauf zu achten, dass die Grundfunktion der Website, nämlich die Information der Benutzenden, stets gewährleistet bleibt.

\section{Spannungsfeld 2: »Alles nach vorne« vs. übersichtliche Inhaltsgestaltung}

In komplexen Organisationen mit zahlreichen Teams und Abteilungen herrscht gerade durch den in Punkt 1 beschriebenen Fokus auf Selbstdarstellung ein gewisser Konkurrenzkampf in Bezug auf Online-Präsenz. Jede Abteilung will an möglichst prominenter Stelle der Website zu finden sein, jede Neuigkeit soll möglichst in der ersten Reihe veröffentlicht werden. In letzter Konsequenz würde dies zu einer Website führen, bei der sich eine derart hohe Zahl von Informationen auf der Startseite findet, dass diese wiederum unbrauchbar wird.

Aufgabe der Webredaktion in diesem Spannungsfeld ist es, eine einigermaßen ausgewogene Inhaltsstruktur zu entwickeln, in der die Informationen aus allen Bereichen gleichwertig und vor allem nachvollziehbar zu finden sind, sowie die Highlights für die Startseite nach Relevanz für die Benutzenden und Zeitaktualität auszuwählen.

\section{Spannungsfeld 3: Gewichtung der Inhalte}

Die Informationsanbietenden besitzen in ihrem Bereich Expertise und umfangreiches Wissen; die Benutzenden hingegen sind Unerfahrene, die ihr Wissen erst durch die bereitgestellten Informationen erwerben müssen. Personen mit Expertise tendieren dazu, möglichst umfangreiche Informationen bereitzustellen, während die Unerfahrenen zuerst auf der Suche nach grundlegenden Informationen sind. Werden sämtliche Informationen zu einem Thema auf einer Webseite angeboten, so werden Unerfahrene nicht nur von der Länge des Textes überwältigt, es fällt auch schwer, zwischen den wesentlichen Informationen und den Details für Sonderfälle zu unterscheiden.

Aufgabe der Webredaktion ist es, sicherzustellen, dass die Inhalte so gewichtet und aufbereitet werden, dass die grundlegende Information für Benutzende priorisiert und stets klar erkennbar ist. 


\section{Spannungsfeld 4: Sprache}

Die von den Personen mit Expertise zur Veröffentlichung auf der Website zur Verfügung gestellten Texte sind oft in einer Sprache verfasst, die Unerfahrenen nicht unmittelbar verständlich sein muss. Ein Problem kann dabei den Benutzenden unbekanntes Fachvokabular sein, oder bei komplexen Sachverhalten auch die Verwendung komplizierter grammatikalischer Strukturen und/oder eines akademischen Stils.

Aufgabe der Webredaktion ist besonders in einem akademischen Kontext, in dem ein gewisses Sprachniveau vorausgesetzt und erwartet wird, trotzdem darauf zu achten, dass Sachverhalte anschaulich erklärt und beschrieben werden und Fachvokabular entweder sparsam eingesetzt oder zumindest so verwendet wird, dass sich seine Bedeutung den Benutzenden unmittelbar erschließt.

\section{Spannungsfeld 5: Bedürfnisse von innen vs. Vorgaben von außen}

Websites von Teilorganisationen bewegen sich meist im Rahmen der Kommunikationsstrategien der übergeordneten Organisation und haben bei der Kommunikation oft deren inhaltliche und/oder gestalterische Vorgaben zu übernehmen, wie z. B. ein Corporate Design. Im Fall von sehr großen und komplexen Teilorganisationen kann der Fall eintreten, dass die für die Mutterorganisation entworfenen Designvorgaben nicht eins zu eins anwendbar sind oder sich gar als kontraproduktiv erweisen. Besonders häufig ist dies der Fall, wenn ein Design, das für die Selbstdarstellung der Organisation (siehe Punkt 1) entwickelt wurde, für die Benutzerinformation eingesetzt werden soll.

Aufgabe der Webredaktion in diesem Spannungsfeld ist, einen Weg zu finden, wie inhaltliche und Designvorgaben so interpretiert und umgesetzt werden können, dass sie die Informationsvermittlung möglichst unterstützen und nicht behindern.

\section{Resümee: Die Webredaktion als Vermittlerin}

Aus diesem kurzen Abriss wird klar, dass sich die Webredaktion stetig zwischen unterschiedlichen Interessen befindet - jenen der Benutzenden, der Mitarbeitenden auf der gleichen Organisationsebene, sowie der übergeordneten Organisation - und dabei die unglückliche Position hat, es nie allen recht machen zu können, da jeder Schritt in eine Richtung die Seite am anderen Ende des Spannungsfeldes benachteiligen würde. Es handelt sich um einen ständigen Vermittlungsprozess, der darauf abzielt, eine funktionierende Kommunikation 
zwischen Informationsanbietenden und Benutzenden zu ermöglichen, sodass letztere die gesuchten Informationen auf möglichst nachvollziehbarem Weg finden.

Wie bereits oben angesprochen arbeitet die Webredaktion in erster Linie für den Benutzenden, um deren durch das Fehlen des direkten Kontaktes verursachte mögliche Defizite beim Auffinden der bereitgestellten Information möglichst zu kompensieren. "Kippt« die Website hin zu einer primären Selbstdarstellung, dann dient sie ab einem gewissen Zeitpunkt nur noch der Kommunikation der Organisation mit sich selbst. Zu helfen, dass dies vermieden wird, ist eine der Schlüsselaufgaben einer guten Webredaktion an komplexen Organisationen wie der Universitätsbibliothek Wien. 
Open-Access-Publikation im Sinne der CC-Lizenz BY-NC-ND 4.0

(c) 2019, Vandenhoeck \& Ruprecht GmbH \& Co. KG, Göttingen ISBN Print: 9783847110989 - ISBN E-Lib: 9783737010986 


\title{
The Times They Are A-Changin'" Zentrales Service Clearingstelle Konsortien, Zentrales Service E-Resource Management
}

\begin{abstract}
"Die Rahmenbedingungen, unter denen Bibliotheken heute ihren Aufgaben nachgehen und ihre Services anbieten, sind durch die digitale Revolution stark verändert. Das gewandelte Umfeld bringt neue Herausforderungen und letztlich den Wandel der Bibliotheken selbst mit sich: Liebgewonnene Gewohnheiten müssen verändert, das Aufgabenspektrum angepasst und womöglich einige Zuständigkeiten über Bord geworfen werden. $\ll^{2}$
\end{abstract}

Als wissenschaftliche Leitbibliothek Österreichs stellt sich die Universitätsbibliothek Wien seit 2004 entschieden diesen Herausforderungen. Die schon 2009 im Strategiepapier Zukunftskonzept der Universitätsbibliothek Wien 2015 formulierte Devise "wo möglich, elektronisch - wo notwendig, print « ist nicht nur eine Ansage zur veränderten Politik des Bestandsaufbaus, sondern vielmehr auch ein Werkzeug tiefgreifender organisatorischer Veränderungen geworden.

\section{From $\mathrm{p}$ to e}

Die nahezu flächendeckende elektronische Verfügbarkeit von wissenschaftlichen Zeitschriften (und Datenbanken) hat zu einer raschen Veränderung der Geschäfts- bzw. Erwerbungsmodelle von einer intellektuellen Auswahl und Erwerbung einzelner Produkte zu der Lizenzierung größerer Pakete und schließlich dem Abschluss sogenannter Big Deals (= „Gesamtpaket eines Anbieters«) geführt.

1 Bob Dylan, (1 1963; Dieser Beitrag basiert allerdings auf dem Vortrag Auswirkungen von neuen Erwerbungs- und Publikationsmodellen auf die Budgets von wissenschaftlichen Bibliotheken am Beispiel der Universitätsbibliothek Wien, gehalten von M. Seissl, B. Kromp und W. Mayer am 15.09.2015 im Rahmen des Österreichischen Bibliothekartags 2015 in Wien.

2 f/21 Büro für Zukunftsfragen (Hg.): Bibliothekswelten im Umbruch. Die Bibliothek im Internetzeitalter (=f/21 Zukunftsperspektiven 08.2016). https://www.f-21.de/downloads/f21_ zukunftsperspektiven_zukunft-bibliotheken.pdf [Letzter Zugriff: 15.01.2019]. 
Ein Meilenstein dieser Entwicklung war die Gründung der Kooperation EMedien Österreich (KEMÖ) ${ }^{3}$ im Jahre 2005. Die Universitätsbibliothek Wien ist im Rahmen dieses Konsortiums an Verträgen mit nahezu allen Großverlagen beteiligt und hat in den Jahren seit 2010 systematisch Printabos in e-only-Subskriptionen umgewandelt oder überhaupt Einzelbestellungen durch den Erwerb von Paketen ersetzt. Während andere Bibliotheken Bedenken zu Big Deals geäußert haben, hat sich für die Universitätsbibliothek Wien aufgrund ihres sehr weiten Benutzer_innenspektrums dieser Weg ökonomisch und im Hinblick auf die Nutzung der bezogenen Inhalte als sehr erfolgreich erwiesen. ${ }^{4}$

Aufgrund der im Unterschied zu E-Journals lange Zeit extrem diversen und unsteten Erwerbungsmodelle bei E-Books wurden hier erst später tiefgreifende Änderungen vorgenommen. Da ein Großteil der zur Einzel- und Paketerwerbung alternativ angebotenen Modelle für die Universitätsbibliothek Wien aufgrund ihrer Größe und Nutzer_innenstruktur zu teuer bzw. riskant gewesen wäre, wurden proaktiv mit einigen Verlagen (Elsevier, De Gruyter ...) und Agenturen (Missing Link) neuartige und maßgeschneiderte Lösungen entwickelt, von denen sich inzwischen einige weltweit am Markt etabliert haben. Dazu gehören im Besonderen verlagsübergreifende Evidence Based Selection (EBS)Modelle, Digital Rights Management (DRM)-freie Zugänge bei Erwerbung über Aggregatoren und neue Kombinationen unterschiedlicher Modelle. Der Plan der Universitätsbibliothek Wien ist, durch den Einsatz einer Vielzahl von tauglichen Modellen eine Gesamt-E-Book-Strategie zu entwickeln, die im Rahmen des Budgets die Wünsche der heterogenen Leser_innenschaft abdeckt.

Ein unmittelbarer Erfolg dieser Aktivitäten war, dass der Anteil des Monographienbestands (aufgrund wachsender Zeitschriften- und Datenbankausgaben jahrelang schrumpfend) wieder bedeutend angewachsen ist.

Für alle Medien gilt allerdings, dass nur durch die konsequente Vermeidung von »Dubletten «, d. h. auch der Vermeidung des Ankaufs von Printversionen der entsprechenden Zeitschriften, Buchreihen und Nachschlagewerke (Stand 2019: nahezu $100 \%$ ) bzw. Einzelmonographien (ca. $90 \%$ ) eine erfolgreiche Bestandsaufbaustrategie umsetzbar ist.

Durch die aktive Mitarbeit in der Gestaltung von Geschäftsmodellen ist die Universitätsbibliothek Wien in den letzten Jahren von einer (sehr) großen Kundin zu einer Partnerin der Anbieter bei der Entwicklung neuer Produkte geworden.

3 https://konsortien.at/ [Letzter Zugriff: 11.09.2019].

4 Der aggregierte Cost per Usage der E-Journal-Big Deals 2017 ergibt bei Ausgaben von $€$ 3.358.198 (inkl. $20 \%$ MwSt) und einer Volltextnutzung laut COUNTER (JR1 minus JR1a [JR5] minus GOA) von 3.302.611 einen Wert von $€ 1,02$. 


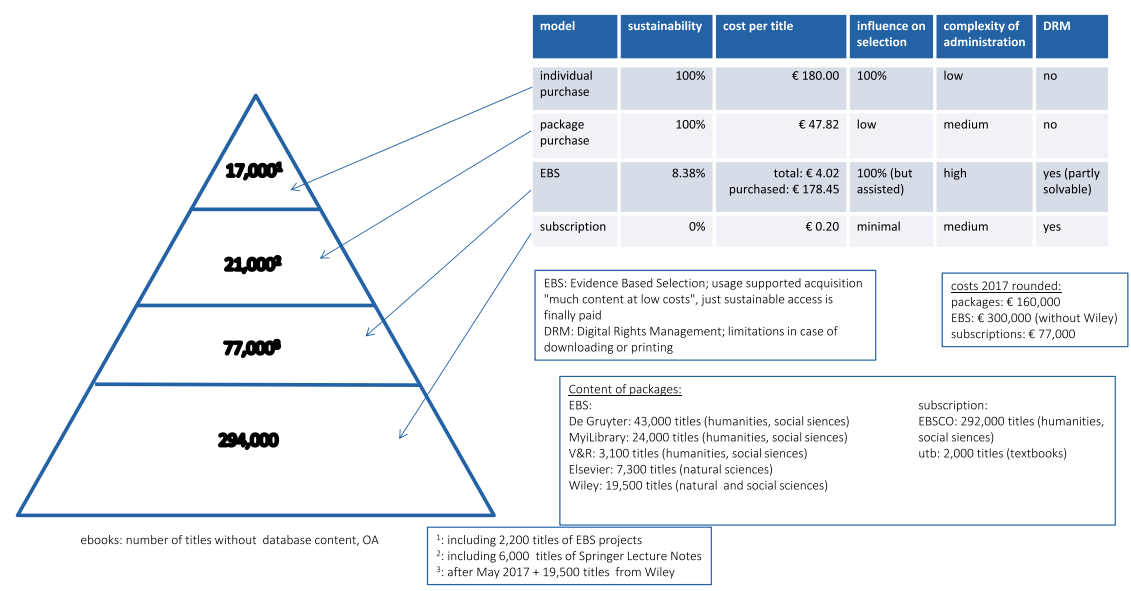

Zahlen beruhen auf dem Budgetjahr 2017, da insbesondere aufgrund des dynamischen Charakters von EBS-Modellen noch keine abgeschlossenen Zahlen für 2018 vorliegen. Die Anzahl der EBS-Titel ist 2018 schon auf 85.500, die der Subskriptionstitel auf 380.000 angestiegen.

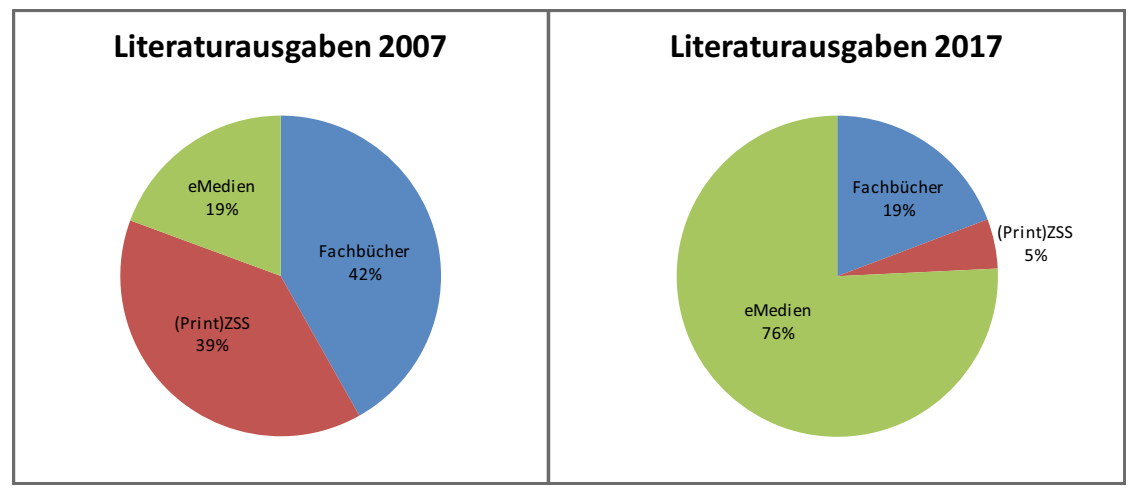

»eMedien« umfassen Datenbanken, E-Journals und E-Book(-Pakete). »Fachbücher« waren 2007 tatsächlich (fast) ausschließlich Printbücher, 2017 sind in dieser Gruppe auch die über die Standortbudgets einzeln erworbenen E-Books enthalten. »(Print)ZSS « enthalten auch Printabos, in deren Rahmen campusweite Online-Zugriffe bezogen werden. Insofern ist der faktische Anteil aller E-Ressourcen an den Literaturausgaben noch höher. 


\section{Von Lizenzierung zu Open Access! $!^{5}$}

Ein weiteres Beispiel für die innovative Rolle der Universitätsbibliothek Wien ist die Entwicklung von sogenannten Transformationsverträgen, die den sukzessiven Wechsel von einer subskriptions- zu einer Open Access-basierten Publikationskultur ermöglichen sollen.

Hier gelang es der Universitätsbibliothek Wien in Zusammenarbeit mit der KEMÖ und dem Fonds zur Förderung der wissenschaftlichen Forschung, 2014 den weltweit ersten sogenannten Offsetting-Deal mit dem Institute of Physics zu entwerfen und in die Realität umzusetzen. Dieses Abkommen erregte internationale Aufmerksamkeit und unter den vielen Gratulationen sei hier die der Max Planck Digital Library stellvertretend angeführt: »... ich gratuliere zur Vereinbarung mit IOP. Das wird weiteren Schwung in die Debatte bringen und setzt ein ganz starkes Signal in die richtige Richtung! ${ }^{6}$

Seit diesem ersten Vertrag wurde konsequent das Ziel verfolgt, bei Verhandlungen mit Verlagen eine Open-Access-Komponente zu integrieren, die es Universitätsangehörigen ermöglicht, ihre Artikel in renommierten Journals ohne Zusatzkosten Open Access zu publizieren.

Diese Aktivitäten der Universitätsbibliothek Wien, vor allem im Rahmen der KEMÖ, haben dazu geführt, dass die österreichischen Universitätsbibliotheken bis 2019 neun unterschiedlich ausgestaltete Transformationsverträge abgeschlossen haben und damit zu den Innovationsführern im Bereich Open Access gehören. Sie sind damit auf die bevorstehenden Entwicklungen innerhalb der Europäischen Union (z. B. Plan $S^{7}$ ) gut vorbereitet.

\section{Von Bestandsaufbaustrategie zur Organisationstransformation}

Nur eine konsequente E-Strategie mit der Zurverfügungstellung vieler Volltextressourcen und entsprechender Tools ermöglicht die Einrichtung neuer forschungsunterstützender Services (Open Access Office, Bibliometrie, Forschungsdokumentation mit CRIS-Systemen ...) einerseits und die Optimierung von herkömmlichen Aktivitäten (Stellplatzbewirtschaftung, Materialschonung, Document Delivery, Informationsvermittlung ...) andererseits.

Bedingung dafür ist die fortlaufende Investition in die Veränderung anderer (konventioneller) Bibliotheksservices, wie z.B. Formalerschließung, Nutzung

5 Siehe auch den Beitrag von Guido Blechl in diesem Band.

6 E-Mail Ralf Schimmer (Director Scientific Information Provision, Max Planck Digital Library) an Brigitte Kromp, 09.02.2014.

7 Science Europe: cOALition S, https://www.coalition-s.org/ [Letzter Zugriff: 15.01.2019]. 
und Priorisierung von Funktionalitäten des Bibliothekssystems, vergaberechtskonforme Erwerbung (auch und besonders von Printmaterialien), Koordination des Bestandsaufbaus unterschiedlicher Standorte, neue Schwerpunkte in den physischen und digitalen Informationsvermittlungsstellen und Evaluation der Bibliotheksservices durch Messung von Nutzung und Benutzer_innenverhalten bzw. -zufriedenheit.

Im Besonderen der Bereich des weitgehenden Ersatzes von Printbüchern durch E-Books wird weniger selbstverständlich von Nutzer_innen angenommen, als das bei anderen Produkten der Fall ist. Das hat oft genannte kulturelle, emotionale und nicht selten auch objektive Ursachen. Dem muss durch die Verbesserung der Benutzungsbedingungen (z. B. »Wegverhandeln von DRM«), kompetente und breit aufgestellte Kommunikation und Information durch Kolleg_innen im Benutzer_innenkontakt und natürlich der Offenheit für die bedarfsmäßige Printerwerbung begegnet werden.

Ein spezifisches Einsatzgebiet der Erwerbung von E-Ressourcen, dem nicht nur aufgrund der spezifischen Platzprobleme der Universitätsbibliothek Wien besonderes Augenmerk gehört, ist der Ankauf von Backfiles sowohl von EJournals als auch von E-Books. Dies nicht nur unter dem Aspekt eines immerwährenden, gesicherten Zugriffs, sondern auch wegen der deshalb möglichen Aussonderung von Printdubletten und der sicheren Archivierung von deduplizierten Beständen, was wiederum zur massiven Reduktion von Binde- und Stellplatzkosten bzw. von Geschäftsgängen führt. In diesem Zusammenhang hat die Universitätsbibliothek Wien auch zwei institutionsübergreifende Initiativen ins Leben gerufen: Shared Archiving Austria ${ }^{8}$ als gemeinsame nationale Archivierungsstrategie aller österreichischen Universitätsbibliotheken und EPICo (European Print Initiatives Cooperation) ${ }^{9}$ zum internationalem Erfahrungsaustausch.

Herausforderungen auf dem Weg zu einer optimierten Umsetzung und Weiterentwicklung dieser Aktivitäten sind unter anderen die Zentralisierung diverser Services (Erwerbung, Metadatenmanagement ...), die Erweiterung und Vertiefung der Kompetenzen der Kolleg_innen an den Standorten der Universitätsbibliothek Wien im Umgang mit E-Ressourcen und die Zugänglichkeit und Transparenz nach innen und außen.

8 https://ubifo.at/sharedarchiving.html [Letzter Zugriff: 11.09.2019].

9 http://www.varastokirjasto.fi/epico/ [Letzter Zugriff: 11.09.2019]. 


\section{Resümee}

In den Jahren 2004-2018 wurden massiv strategische Akzente gesetzt, um Ressourcen und Services der Universitätsbibliothek Wien zu digitalisieren. Um diese (de facto ja auch kaum umkehrbaren) Änderungen nicht nur nachhaltig zu sichern, sondern vielmehr auch stetig auszubauen und flexibel für die immer neuen und sich immer schneller ändernden Herausforderungen des Wissenschaftsbetriebs vorzubereiten und fit zu machen, braucht es:

- aufmerksames Monitoring und proaktives Gestalten der neuen Entwicklungen, auch mit dem Mut, Ressourcen in Testszenarien zu investieren

- durchgehende Kompetenzentwicklung und Aufgabentransformation der Mitarbeiter_innen, um selbstsicher und fokussiert in ihre jeweilige Benutzer_innengruppe hineinhören und -wirken zu können

Die beschriebenen Entwicklungen verlangen von den Mitarbeiter_innen der Universitätsbibliothek Wien hohe Bereitwilligkeit zu Veränderung und Leistung, aber zugleich von der Bibliotheksleitung den Mut, auch mit dem Risiko des Scheiterns neue Pfade zu beschreiten. Maria Seissl ist beides gelungen: Die Motivation zu Leistungsbereitschaft und Offenheit für Veränderungen bei ihren Mitarbeiter_innen zu wecken und Neugestaltungen der Bibliotheksservices überzeugend nach außen zu vertreten. 


\section{Filialen, Shopping-Welt und Retro-Chic. Der Wandel des Teams Integrierte Medienbearbeitung Fachbereichsbibliotheken}

In den letzten zwei Jahrzehnten erlebte die Abteilung »Zentrale Katalogisierung der Institute« (ZKI) nicht nur eine Namensänderung in »Team Integrierte Medienbearbeitung Fachbereichsbibliotheken« (TIM), sondern auch einen Wandel ihres Selbstverständnisses und ihres räumlichen und inhaltlichen Tätigkeitsbereichs.

\section{Ein neues Selbstbild}

Als 1989 mit dem Bibliothekssystem BIBOS die neue Ära des Online-Katalogs begann, wurde die Kernaufgabe der 1972 als »Zentrale Katalogisierung der Institute« an der Hauptbibliothek der Universitätsbibliothek Wien eingerichteten Abteilung, zum Zweck eines Gesamtüberblicks über die neu angeschafften Bestände der Fachbereichsbibliotheken der Universitätsbibliothek Wien (der ehemaligen Institutsbibliotheken) einen zentralen Kartenkatalog zu führen, obsolet, da ab diesem Zeitpunkt deren Bestände gemeinsam mit denen der Hauptbibliothek und sogar denen des Österreichischen Bibliothekenverbundes im Online-Katalog recherchiert werden konnten. Die Rolle der Abteilung änderte sich dadurch fast unmerklich zu der einer Service-Abteilung, die für Fachbereichsbibliotheken, die diese Unterstützung benötigten, die Formalerschließung der Neuzugänge übernahm.

\section{Neue Räume}

Um kürzere Transportwege und damit eine schnellere Bearbeitung der neu angeschafften Literatur zu ermöglichen, wurden im Laufe der Zeit für die Abteilung Außenstellen in größerer Nähe zu den Fachbereichsbibliotheken geschaffen. Die erste Gelegenheit dazu bot sich im Oktober 1997, als der Campus der Universität Wien (das ehemalige Alte $\mathrm{AKH}$ ) von geisteswissenschaftlichen Universitätsin- 
stituten samt ihren Fachbereichsbibliotheken besiedelt wurde und anfangs zwei, später sechs Mitarbeiter_innen der Abteilung dankenswerterweise in Räumen der Fachbereichsbibliothek Anglistik und Amerikanistik "Herberge " fanden. Durch diese "Kundennähe« können seither die elf Campus-Bibliotheken einschließlich der in Campusnähe verorteten Fachbereichsbibliothek Publizistikund Kommunikationswissenschaft und Informatik noch besser unterstützt werden. Aufgrund dieses Erfolgsmodells wurden seither weitere Außenstellen, meist ebenfalls im Zuge von Übersiedlungen und Zusammenlegungen von Fachbereichsbibliotheken, im Neuen Institutsgebäude (NIG), an der Fachbereichsbibliothek Bildungswissenschaft, Sprachwissenschaft und Vergleichende Literaturwissenschaft, der Fachbereichsbibliothek Psychologie, der Fachbereichsbibliothek Theater-, Film- und Medienwissenschaft und der Fachbereichsbibliothek Theologie eingerichtet - so wurde die ehemals auch räumlich »Zentrale Katalogisierung« zu einer Abteilung mit einem effizienten »Filialnetz«.

\section{Neue Aufgaben}

Spätestens seit der Einführung des neuen integrierten Bibliothekssystems Aleph im Jahr 1998, mit dem die gesamte Bibliotheksverwaltung durchgeführt werden konnte, sollte auch die Erwerbung flächendeckend damit abgewickelt werden nicht nur, um alle Vorteile einer elektronischen Verwaltung nutzen zu können, sondern auch, um Benutzer_innen des Online-Katalogs sofort Informationen über bestellte Titel zu bieten. Da zu diesem Zweck sofort ein bibliographischer Bestelldatensatz in der Datenbank des Österreichischen Verbundkatalogs erstellt werden musste, landeten die Fachbereichsbibliotheken, die ihre Bestellungen bisher telefonisch, per Fax, Brief oder persönlich an die Buchhändler_innen übermittelt und die darauf folgende Eingangs-, Rechnungs- und Budgetverwaltung sowie eventuelle Mahnungen meist auf Papier verwaltet hatten, plötzlich im Bereich der Formalerschließung und benötigten, sofern an der Fachbereichsbibliothek keine Kapazitäten für diese neuen Tätigkeiten vorhanden waren, nun auch bei der Erwerbung Unterstützung. So erhielt die Abteilung Zentrale Katalogisierung der Institute im Juni 2002 von der Bibliotheksdirektion den zusätzlichen Auftrag, in einem ersten Schritt die Fachbereichsbibliotheken am Uni-Campus auch bei der Beschaffung der Literatur zu unterstützen. Dieser neue Service wurde in den folgenden Jahren auch für zahlreiche weitere Fachbereichsbibliotheken an anderen Standorten übernommen - die bisherigen Formalerschließer_innen erweiterten ihren Horizont hinaus in die "schöne neue Shoppingwelt" und wurden nun auch zu Kenner_innen des internationalen Buchhandels, von Verlags-Webseiten, Preisgestaltung, Verrechnung und Budgetverwaltung, Vergaberecht und seit einigen 
Jahren auch zu Rechnungsprüfer_innen innerhalb des SAP-Rechnungsworkflows des Finanzwesens der Universität Wien. Durch die Bearbeitung "aus einer Hand «, aber auch durch die noch engere Zusammenarbeit mit den Fachbereichsbibliotheken konnten zahlreiche Synergien genutzt werden. 2005 kam es in der Folge zur Umbenennung in »Team Integrierte Medienbearbeitung Fachbereichsbibliotheken " (TIM) und auch zur organisatorischen Änderung von einer Abteilung der Hauptbibliothek zu einem Zentralen Service der Universitätsbibliothek.

\section{Neue Ziele}

Frage: Was würden sich Formalerschließer_innen einer Abteilung, die einst mit dem Ziel, einen Zentralkatalog zu erstellen, gegründet wurde, von einer guten Fee wünschen? Antwort: Der Gesamtbestand der Universitätsbibliothek Wien soll in der Suchmaschine u:search recherchierbar sein und die Fragen fassungsloser Bibliotheksbenutzer_innen »Sind leicht nicht alle Bücher der Universitätsbibliothek in u:search?! Wie finde ich die denn dann? « sollen damit endlich der Vergangenheit angehören! Um dieses Ziel zu erreichen, wurde in den letzten 15 Jahren in zwei großen und zahlreichen kleineren Projekten intensiv an der retrospektiven Erfassung der Altbestände gearbeitet.

2006 entschied man sich bei dem Projekt »Retrokonversion der Nominalkataloge der dezentralen Bibliotheken" im Gegensatz zu dem seit 2004 an der Hauptbibliothek laufenden Projekt "Retrokonversion des Nominalkatalogs 1932-1988 der Hauptbibliothek « aufgrund der mangelhaften Qualität der älteren Kartenkataloge an den Fachbereichsbibliotheken gegen eine Fremdfirma und stattdessen für eine Erfassung durch Autopsie. Die infrage kommenden Bestände wurden aufgrund ihrer Nutzungsfrequenz, ihrer Einzigartigkeit und der Anzahl der Studierenden im jeweiligen Fachbereich ausgewählt. Das TIM wurde von Mai 2006 bis Dezember 2011 durch ein sechsköpfiges Projektteam ergänzt, das rund 251.000 Titel retrospektiv online erfasste. Seit 2012 konnte durch eine personelle Aufstockung des TIM die Retrobearbeitung intensiv weitergeführt werden, Retro wurde nun endgültig chic! Besonders hervorzuheben ist dabei das im April 2013 begonnene Projekt »UB-Maps«, die Erschließung von bisher rund 12.500 Kartenblättern der Fachbereichsbibliothek Geographie und Regionalforschung, die in Zukunft, sofern urheberrechtsfrei, auch digital zur Verfügung stehen werden - doch das ist eine andere Geschichte ... ${ }^{1}$ Noch wurde der Wunsch nach einer Erfassung des gesamten Bestandes nicht ganz erfüllt, doch durch die Unterstützung der "guten Fee« konnten von 2012 bis 2018 gemeinsam mit den

1 Siehe dazu den Beitrag von Alexandra Gappmayr in diesem Band. 
Kolleg_innen der Abteilung Formalerschließung der Hauptbibliothek rund 325.500 weitere Titel online nachgewiesen werden - und täglich werden es mehr!

Und so unterstützt das »Team Integrierte Medienbearbeitung Fachbereichsbibliotheken" 2019 einerseits als engagierte Serviceabteilung beinahe alle 40 Fachbereichsbibliotheken ganz oder teilweise in den Bereichen Medienerwerbung und -erschließung, arbeitet intensiv mit bei der Erreichung des Ziels, den Gesamtbestand der Universitätsbibliothek Wien retrospektiv online zu erfassen, und erwartet aufgrund seiner Erfahrungen der letzten Jahre auch zukünftigen Wandel und damit einhergehende Herausforderungen mit Neugier und Gelassenheit. 


\section{Die »bibliothekarische Kränkung «. Der Weg von einer Abteilung der Hauptbibliothek hin zum Zentralen Service Team Sacherschließung}

Freud möge gnädig sein, dass hier seinen drei Kränkungen der Menschheit eine vierte hinzugefügt wird: die Bibliothekarische. Gemeint wäre damit - in Anspielung auf Freuds Begriff der kosmologischen Kränkung (die Erde ist nicht mehr Mittelpunkt des Universums) - ein neuer Blick auf das »bibliographische Universum«, der die eigenen Bestände und selbst erstellten Datensätze nicht mehr im Mittelpunkt des Katalogs, die Bibliothekar_innen nicht mehr als "Herr_innen « der Inhalte und Funktionen ihrer Rechercheangebote sieht. Geht man Freuds Begriff der kosmologischen Kränkung nach, so stößt man aber bald auch auf den Begriff der kopernikanischen Wende. Es ist also eine Frage des Standpunktes, ob man die im Folgenden beschriebenen fundamentalen Änderungen als Kränkung oder als Wende betrachtet. Man könnte also auch von einer "bibliothekarischen Wende" sprechen.

Diese Wende im betrachteten Zeitraum von zehn Jahren führt von klar der Bibliothek und ihren Bearbeiter_innen zuordenbaren Beständen im OnlineKatalog hin zu riesigen Datenmengen in einer Suchmaschine; sie führt von in einem Regelwerk klar definierten Normdaten zu einem gemeinsamen Datenpool, aus dem jeder das richtige herausangeln muss; und von einem gut angepassten Arbeitsinstrument $\mathrm{zu}$ einem plastischen System, das sich monatlich ändert und für Überraschungen sorgt.

Die inhaltliche Erschließung war im Jahr 2008 als Abteilung der Hauptbibliothek organisiert, die von Katalin Vanyai geleitet wurde. Zu dieser Zeit eröffneten sich zusätzliche Aufgabenfelder wie "Aus- und Fortbildung", die in die Verantwortung der neu zur Abteilung geholten Kolleginnen Andrea Neidhart und Martina Cuba, und »klassifikatorische Erschließung«, die Kurt Schaefer und Sandra Mann übertragen wurde. Für die Unterstützung der Arbeit der Lokalredaktion kam 2009 Stefan Alker-Windbichler hinzu. Zu dieser Zeit wurden an der gesamten Universitätsbibliothek sogenannte "Zentrale Services« eingerichtet. Da inhaltliche Erschließung zu einem wesentlichen Teil auch in den Fachbereichsbibliotheken stattfindet, hatte die Abteilung der Hauptbibliothek auch Hilfestellung in diesem Bereich zu bieten - eine Umwandlung in ein Zen- 
trales Service war naheliegend. 2010 übernahmen viele der genannten Personen andere wichtige Funktionen. Daher bemühte man sich, interessierte Kolleg_innen aus dem Kreis der Referent_innen und Fachbereichsbiliotheks-Leiter_innen für das Team zu gewinnen. Das führte 2014 dazu, dass sich zu dieser Zeit viele Namen im Organigramm fanden. Der Input von dieser Seite war aber gering und die große Gruppe war zu träge. So formierte sich 2017 ein Kernteam, das sich auch stark bei der Implementierung von Alma engagierte. Der Ruhestand von Katalin Vanyai 2018 machte eine Erneuerung im Team notwendig hinzu kamen Sonja Hasslehner-Wimmer und Sonja Fiala.

Die inhaltliche Erschließung bestand 2008 an der Universitätsbibliothek Wien im Wesentlichen nur aus Schlagwortketten, die nach den Regeln für die Schlagwortkatalogisierung (RSWK) erstellt wurden. Während Fachbereichsbibliotheken oft eigene Aufstellungssystematiken weiter pflegten, hatte die Hauptbibliothek die sogenannte "Peschl-Systematik«, das seit den 1970er Jahren eingesetzte systematische Schlagwortregister von Otto Peschl, schon seit einigen Jahren aufgegeben. Im Österreichischen Bibliothekenverbund (OBV) regte sich Interesse an Klassifikationssystemen und unser Team führte Tests zur Verwendung der Basisklassifikation (BK), Workshops zur Dewey Decimal Classification (DDC) und Vorträge zur Regensburger Verbundklassifikation (RVK) durch. Das 2009 in Wien an der Österreichischen Nationalbibliothek abgehaltene Symposium der European DDC Users Group (EDUG) und der anschließende Besuch der Herausgeberin der DDC Joan Mitchell beflügelte den Wunsch nach Einführung und Verwendung der DDC an der Universitätsbibliothek Wien. Die Entscheidung über ein in der ganzen Universitätsbibliothek gültiges Klassifikationssystem gipfelte in der salomonischen Frage: Wollt ihr BK oder wollt ihr BK? Seither dient die Basisklassifikation an der Universitätsbibliothek als zusätzliches Erschließungselement im Sinne eines »broader terms". Unseren Wünschen nach der DDC wurde insofern nachgegeben, als DDC aus Fremddaten übernommen werden kann, die Universitätsbibliothek der EDUG beigetreten ist und so 2010 beim außereuropäischen EDUG-Meeting in Alexandria vertreten war. Außerdem wurde 2011 die Planung einer Neuaufstellung der Bestände im Großen Lesesaal der Hauptbibliothek begonnen. 2013 wurde der Lesesaalbestand des Fachbereichs Theologie nach DDC aufgestellt. Darüber und über die schwierige Situation der DDC in Österreich wurde beim EDUG-Meeting 2014 in Reykjavik berichtet. 2015, zum 650-Jahr-Jubiläum der Universität, sollte die Neuaufstellung fertig sein. Die Verantwortung wurde der Abteilung Fachreferate der Hauptbibliothek übertragen. Nur einige Fachbereiche wurden überarbeitet und nach DDC klassifiziert, dann kam das Projekt zu erliegen.

Ein stetes Bemühen galt auch der Nutzbarkeit der produzierten Metadaten. So konnte 2009 im Online-Katalog eine thematische Suche etabliert werden, die Schlagworte, Schlagwortfolgen und Klassifikationen als Indices und Suchein- 
stiege anbot. Trotz zahlreicher Änderungen dieser Funktionen gaben solche Suchen bis zur Ablöse durch die neue Bibliothekskatalog-Software "Primo" (bzw. u:search) 2016 ein eindeutig definiertes Ergebnis. Das Prinzip der thematischen Facette in Primo veränderte die Präzision der Filterung nach Schlagworten und anderen inhaltlichen Metadaten stark. Besonders augenfällig wurde dies, als vom Buchhandel vergebene Schlagworte eingespielt wurden: Die dynamische Facette - sie reiht die häufigsten Begriffe ganz oben - ergab »Softcover« als häufigstes »Thema«. In Zusammenarbeit mit dem Systemteam und den Kolleg_innen von der Formalerschließung wurde in Arbeitspapieren die Wechselwirkung von bibliographischen Daten im Bibliothekssystem Aleph und dem Discovery-System Primo beleuchtet. Seit dem Wechsel des bibliographischen Datenformats vom deutschsprachigen MAB zu MARC 21 im Jahr 2016 und dem Bibliothekssystem Alma 2017 ist solch eine genaue Analyse wieder erforderlich.

Alma forderte unsere Gruppe seit Anbeginn. Vier Mitglieder des Teams waren seit 2016 bei den Vorbereitungen, 2017 beim Testbetrieb, den Konverter- und Abnahmetests sowie im sogenannten "First-Level-Support « beteiligt. Die monatlichen Releases werden in den Jour Fixes der Arbeitsgruppe "Alma und Resource Management (Metadaten) « besprochen, untersucht und analysiert. Auf die in der Praxis mitunter unerwarteten Auswirkungen muss oft rasch reagiert werden. Ein Zustand, der uns anscheinend für die Dauer des Betriebs von Alma erhalten bleibt.

Besser davongekommen ist die Sacherschließung bei zwei weiteren grundlegenden Änderungen: Der Umstellung auf das Format MARC (die zugewiesenen Kategorien für Klassifikationen, Formangaben und Schlagworte sind überschaubar und bringen wenig Neuerungen) und der Einführung des Regelwerkes Resource Description and Access (RDA). 2014 war der Bereich der inhaltlichen Erschließung dort nur angekündigt, 2018 war klar, dass in der künftigen RDA inhaltliche Erschließung gar nicht vorkommen wird und wir mit den RSWK weiterarbeiten werden. Bei der Normdatenarbeit erreichte uns aber ein Teil der RDA - einige Entitäten der Sacherschließung werden nach RDA erfasst. Ursprünglich war die Grundidee, dass alle Fachreferent_innen, die inhaltlich erschließen, auch Daten in der Normdatei erfassen können. Da der Komplexitätsgrad mit der Regelwerksänderung deutlich anstieg, entschloss man sich 2014, die Schreibberechtigungen in der Normdatei zu begrenzen und allen anderen ein Online-Formular für die Neuansetzung oder notwendige Anpassung von Schlagworten anzubieten. Die im Team Sacherschließung angesiedelte Lokalredaktion Sacherschließung bearbeitet dann die erforderlichen Datensätze nach den RSWK, den Übergangsregeln bzw. RDA. Seit der Neuorganisation der Zentralen Redaktion im OBV 2016 ist auch ein Teil der Level-1-Redaktion - sie kontrolliert und korrigiert Datensätze und versieht sie mit dem »höchsten« 
Katalogisierungslevel 1 - an der Universitätsbibliothek vertreten. In unserem Team werden gemeinsam mit anderen Verbundbibliotheken Sachschlagworte, Geographika und Werktitel betreut. Die Kolleg_innen von diesen Bibliotheken sind es auch, mit denen Vertreter_innen unseres Teams in Gremien - der Zentralen Redaktion und dem Teilgremium Sacherschließung des OBV - zusammenarbeiten. Im Zuge von Veranstaltungen dieser Gremien werden uns auch künftige Entwicklungen wie automatische Erschließung und der "Digitale Assistent « - ein Tool, das passende Schlagworte und Klassifikationen vorschlägt vorgeführt. Unser wachsames Auge bleibt aber jedenfalls auch weiterhin gefragt. 


\section{Erinnerungsarbeit, Restitution und historische Verantwortung. Ein Überblick zum Arbeitsbereich NS-Provenienzforschung ${ }^{\top}$}

Die Auseinandersetzung mit der Geschichte des Nationalsozialismus begann an der Universität Wien erst relativ spät. Heute bekennt sich die Universität zu ihrer Rolle und Mitschuld im nationalsozialistischen Regime und kommt der Verantwortung nach, sich kritisch mit der eigenen Geschichte der Jahre 1933-1945 und folgend auseinanderzusetzen. Diese Verpflichtung erfüllt sie in Lehre und Forschung seit den 1980er Jahren zunehmend, wobei die NS-Provenienzforschung der Universitätsbibliothek Wien ein Teil der vielfältigen Forschungs- und Gedenkprojekte der Universität Wien ist und einen aktiven Beitrag zur Erinnerung an die Opfer des Nationalsozialismus leistet.

\section{Systematische Erfassung und Erweiterung der Forschung}

Als erste Universitätsbibliothek in Österreich begann die Universitätsbibliothek Wien 2004 mit der systematischen Durchsicht und der wissenschaftlichen Aufarbeitung der eigenen Erwerbungspolitik. Ziel war es, zunächst die Hauptbibliothek und ab 2005 die Fachbereichs- und Institutsbibliotheken auf bedenkliche Erwerbungen der Jahre 1938-1945 zu überprüfen. Dieser Auftrag musste bald sowohl hinsichtlich des Erwerbungszeitraums als auch in Bezug auf die beforschten Objekte erweitert werden.

Zwischen 2004 und 2009 wurden über 400.000 Bücher autopsiert, die im Untersuchungszeitraum in die Universitätsbibliothek Wien (Haupthaus und Fachbereichsbibliotheken) eingegangen waren. Dabei handelte es sich jedoch nicht nur um Eingänge bis 1945 . Während die inzwischen als notwendig erkannte zeitliche Ausdehnung der Untersuchungen auf die Jahre 1933-1938 bis-

1 Der Beitrag beruht zum Teil in zusammengefasster und ergänzter Form auf Olivia Kaiser, Markus Stumpf: Provenienzforschung in der Universitätsbibliothek, dem Universitätsarchiv und den musealen Sammlungen der Universität Wien. In: Eva Blimlinger, Heinz Schödl (Hg.): ... (k)ein Ende in Sicht. 20 Jahre Kunstrückgabegesetz in Österreich. Wien, Köln, Weimar: Böhlau 2018 (= Schriftenreihe der Kommission für Provenienzforschung 8), S. 187-204. 
lang nur partiell an einigen Fachbereichsbibliotheken durchgeführt werden konnte, wurde der Untersuchungszeitraum weit über das Ende des Zweiten Weltkrieges hinaus ausgedehnt, um dem über die Büchersortierungsstelle an der Österreichischen Nationalbibliothek an die Universitätsbibliothek Wien gelangten Bestand, der intern als sogenannte "Sammlung Tanzenberg « bezeichnet wurde, sowie antiquarischen Erwerbungen nachgehen $\mathrm{zu}$ können. ${ }^{2}$ In den Nachkriegsjahren erfolgten zwar einige wenige Rückgaben in Fällen, in denen sich Überlebende aktiv an die Universitätsbibliothek Wien wandten. Diese Rückgaben wurden jedoch nicht aus Unrechtsbewusstsein von Seiten der Bibliotheksleitung durchgeführt - galt es doch »Kriegsverluste« auszugleichen.

Im Zuge der Forschungen rückte auch das Archiv der Universität Wien als eigenständige Sammlung in den Fokus der NS-Provenienzforschung an der Universitätsbibliothek Wien. Auf Wunsch der Universitätsleitung wurde daher im Herbst 2007 die NS-Provenienzforschung auf das Universitätsarchiv Wien ausgeweitet. Nachdem aus dem Projekt im Jahr 2010 ein eigener verstetigter Arbeitsbereich $^{3}$ entstand, wurde auch die Bandbreite der Untersuchungsgegenstände erweitert, d.h. nach den Bibliotheks- und Archivbeständen rückten nun auch die wissenschaftlichen Sammlungen der Universität Wien zunehmend in den Fokus der Provenienzforschung. Dieser Prozess erfolgte gegenläufig zu den Bundesmuseen, die mit Ausnahme der Österreichischen Nationalbibliothek von Kunstobjekten ausgingen und erst langsam die geraubten Bücher der jeweiligen Museumsbibliotheken in der Forschung berücksichtigten.

\section{Nationale und internationale Vernetzung}

Seit Beginn der Forschung kooperiert der Arbeitsbereich mit nationalen und internationalen Institutionen und Organisationen und ist dabei bestens vernetzt. So arbeitet der Arbeitsbereich auf universitärer Ebene mit verschiedenen Wissenschaftsbereichen eng zusammen, z. B. mit dem Institut für Zeitgeschichte und dem Forum »Zeitgeschichte der Universität Wien«. Auch ist der Arbeitsbereich in der Kommission für Provenienzforschung im Bundeskanzleramt der

$2 \mathrm{Zu}$ den letzten Ergebnissen zur Sammlung Tanzenberg vgl. Markus Stumpf, Christina Köstner-Pemsel, Olivia Kaiser: »Treuhänderisch« - Themenaufriss im Kontext der NS-Provenienzforschung. In: Olivia Kaiser, Christina Köstner-Pemsel, Markus Stumpf (Hg.): Treuhänderische Verwahrung und Übernahme - international und interdisziplinär betrachtet. Göttingen: Vienna University Press/V\& R unipress 2018 (= Bibliothek im Kontext 3), S. 37-53. DOI: https://www.vr-elibrary.de/doi/pdf/10.14220/9783737007832.37.

3 Das Team des Arbeitsbereiches Provenienzforschung setzt sich Ende 2018 aus folgenden Personen zusammen: Stefan Alker-Windbichler, Olivia Kaiser, Christina Köstner-Pemsel, Karin Lach, Monika Schreiber, Markus Stumpf (Leitung) und Susanne Wicha. Den Kolleg_innen sei an dieser Stelle für die gute Zusammenarbeit herzlich gedankt! 
Republik Österreich vertreten. 2008 wurde die Arbeitsgemeinschaft »NS-Provenienzforschung" der Vereinigung Österreichischer Bibliothekarinnen und Bibliothekare initiiert, deren Leitung seitdem Provenienzforscher_innen der Universitätsbibliothek Wien wahrnehmen. 2013 war der Arbeitsbereich Mitinitiator des in Deutschland begründeten Arbeitskreises »Provenienzforschung \& Restitution - Bibliotheken«. Auch wird in den entsprechenden Gremien des Österreichischen Bibliothekenverbundes zur Provenienzerschließung mitgearbeitet, wobei die öffentliche Ausweisung der restituierten Werke als "Stolpersteine im Publikumskatalog als eine Form der Erinnerungsarbeit von besonderer Bedeutung ist. Dabei werden der NS-Raub und die ursprüngliche Provenienz ausgewiesen. Diese Information bleibt auch nach Restitution der Werke such- und sichtbar. Im Zuge der Erbensuche wird der Arbeitsbereich durch die Restitutionsabteilung der Israelitischen Kultusgemeinde Wien sowie durch den Nationalfonds der Republik Österreich für Opfer des Nationalsozialismus unterstützt.

Zusätzlich sorgte der Arbeitsbereich für interne und externe Sensibilisierung für dieses Thema und trug durch zahlreiche nationale und internationale Vorträge, Ausstellungen sowie der Ausrichtung von internationalen wissenschaftlichen Tagungen (2008: Bibliotheken in der NS-Zeit - Provenienzforschung und Bibliotheksgeschichte; 2013: Guido Adlers Erbe - Restitution und Erinnerung an der Universität Wien; 2017: "Treuhänderische" Übernahme und Verwahrung international und interdisziplinär betrachtet) und entsprechende Veröffentlichungen zur wissenschaftlichen Forschung bei.

\section{Ergebnisse}

Im Zuge der Forschungen wird die enge Verwobenheit von Personen, Instituten, Bibliotheken, Sammlungen und Archiven der Universität Wien inner- und außerhalb der Universität sichtbar. So ist etwa festzuhalten, dass es für den Zeitraum von 1933 bis 1945 an der Universität Wien aufgrund des historisch gewachsenen, mehrschichtigen Bibliothekssystems eine heute nicht mehr genau feststellbare Anzahl an Bibliotheken und Büchersammlungen gab. Denn neben der Universitätsbibliothek (= heutige Hauptbibliothek) gab es Instituts- und Seminarbibliotheken, aber auch eingelagerte Privatbibliotheken und diverse Büchersammlungen. Alleine an der Philosophischen Fakultät sind in diesem Zeitraum zumindest 51 Bibliothekssammlungen feststellbar. Zwar konnte für die systematische Suche und wissenschaftliche Aufarbeitung der eigenen Erwerbungspolitik bereits auf einige Forschungsergebnisse aus dem Bereich der Wissenschaftsgeschichte zurückgegriffen werden, jedoch fehlen für die Hauptbibliothek, vor allem aber für die zahlreichen Fachbereichsbibliotheken und 
deren wissenschaftliche Fächer, teils bis heute kritische institutionengeschichtliche Arbeiten, die eine wesentliche Basis für eine Einordnung der erhobenen Daten bilden. Neben der Hauptbibliothek liegen für die Bibliotheken der Fachbereiche Anglistik, Europäische Ethnologie (Volkskunde), Judaistik, Kunstgeschichte, Musikwissenschaft, Orientalistik, Philosophie, Psychologie, Romanistik und Theaterwissenschaft sowie für das Archiv der Universität Wien und für Bestände der Sammlungen der Universität Wien aus den Fachbereichen der Ägyptologie, Musikwissenschaft und Zoologie erste Ergebnisse vor. Damit trägt die NS-Provenienzforschung zur Erforschung des Nationalsozialismus über das Thema des Kulturgüterraubes hinaus auch zur Wissenschaftsgeschichtsschreibung bei.

Im Jahr 2009 wurden die ersten Restitutionen durchgeführt und bis Ende 2018 konnten von der Universitätsbibliothek Wien 25 Restitutionsfälle mit insgesamt etwa 2.250 Druckwerken, fünf Gipsabdrücken und einem literarischen Splitternachlass durchgeführt werden. In weiteren 22 Fällen werden die Erb_innen gesucht und 24 Verdachtsfälle erwiesen sich als rechtmäßige Erwerbungen. In fünf weiteren Fällen werden zur Klärung der Erwerbungsgeschichte noch zusätzliche Informationen benötigt, daher wurden die Objekte in die Kunstdatenbank des Nationalfonds eingetragen. In zahlreichen weiteren Fällen wurde mit der Forschung begonnen. Einen Meilenstein für den Umgang mit sogenanntem »erblosen « Gut stellte ein Vertrag mit dem Nationalfonds dar, der im April 2017 geschlossen werden konnte. Dadurch werden jene Bücher und Objekte, für die keine Rechtsnachfolger gefunden werden können, dem Nationalfonds zur Verwertung übergeben. In weiterer Folge werden diese von der Universität angekauft, um mit dem Erlös im Sinne des Nationalfonds Opfer des Nationalsozialismus zu unterstützen.

Die Provenienzforschung, die Rückgabe und die Veröffentlichung der Ergebnisse sind dabei als Mosaiksteine der heutigen Erinnerungskultur an der Universität Wien zu sehen. Die eingeschriebenen Geschichten in den beforschten Büchern und Objekten verweisen auf Personen oder Institutionen, deren Existenzberechtigung das nationalsozialistische Regime negierte und die in Folge enteignet oder zwangsweise geschlossen, verfolgt und vernichtet wurden. Die Rückgabe von geraubten Kulturgütern ist daher als Bemühungen um "faire und gerechte« Lösungen im Sinne der Washingtoner Prinzipien zu verstehen. 


\section{Viktoria Lang-Steixner}

\section{Was ein ideales Bibliothekssystem können sollte. Die wechselvolle Arbeit der Arbeitsgruppe Alma}

Kann es ein Bibliothekssystem geben, welches für alle Bibliothekarinnen und Bibliothekare gleichermaßen zufriedenstellend ist? Dass diese Frage wohl kaum mit einem Ja beantwortet wird, liegt nicht zwingend nur an den unterschiedlichen Tätigkeiten, die die Anwenderinnen und Anwender des Systems zu bewerkstelligen haben, sondern auch an den individuellen Bedürfnissen, die jeder Mensch zu Recht für die Verrichtung ihrer oder seiner Arbeit mitbringt. Daher ist es nicht überraschend, dass sich auch bei dem neu implementierten Bibliothekssystem Alma der Firma Ex Libris die Geister scheiden.

Alma wurde in einem umfassenden österreichweiten Projekt von Jänner 2016 bis März 2018 an zahlreichen wissenschaftlichen Bibliotheken implementiert. Letzteres Datum markiert dabei den Umstieg der Verbundzentrale und damit die Anbindung aller zu diesem Zeitpunkt bereits migrierten Bibliotheken an die sogenannte Netzwerkzone in Alma. Die Universitätsbibliothek Wien selbst hatte den Implementierungsprozess bereits im August 2017 gemeinsam mit fünf weiteren Bibliotheken erfolgreich abgeschlossen. Das Projektteam an der Universitätsbibliothek Wien, unter der Leitung von Wolfram Seidler und der Projektassistenz durch Olivia Kaiser, bestand zunächst nur aus wenigen Expertinnen und Experten unterschiedlicher Tätigkeitsbereiche. Im Laufe des Projektfortschritts wurde das Team kontinuierlich erweitert. Die neuen Mitglieder unterstützten die Hauptverantwortlichen bei den Herausforderungen des Projekts und gewährleisteten, den Schulungsbedarf von 400 Mitarbeiterinnen und Mitarbeitern abzudecken. Für die Projektkoordination konnte die Direktion der Universitätsbibliothek Wien zusätzliche Mittel organisieren, weshalb hierfür eine temporäre Stelle geschaffen wurde.

Durch vom Projektteam organisierte Informationsveranstaltungen wurden die Mitarbeiterinnen und Mitarbeiter über den Zeitplan der Implementierung in Kenntnis gesetzt und konnten erste Einblicke in das neue Bibliothekssystem gewinnen. Dabei wurden die Teilnehmenden darum gebeten, zu notieren, was sie von einem idealen Bibliothekssystem erwarten. Das Ergebnis hätte kaum un- 
terschiedlicher ausfallen können, auch wenn einige der anonymen Wortmeldungen gut und gerne auch mit einem Augenzwinkern zu verstehen waren.

\section{»Ein ideales Bibliothekssystem kann meine Gedanken lesen«}

Was für die einen utopisch und für die anderen eher angsteinflößend klingt, schien zu diesem Zeitpunkt zumindest für eine Person eine wünschenswerte Arbeitserleichterung gewesen zu sein. Es muss wohl nicht erwähnt werden, dass Alma diese Fähigkeit nicht besitzt, jedoch ist es durchaus denkbar, dass einige Mitglieder des Projektteams während der Projektphase einen ähnlichen Wunsch hatten - wobei dieser wohl eher lautete, die Gedanken von Alma lesen zu können. Das Projektteam musste sich nämlich den Großteil der Alma-Kenntnisse im Selbststudium aneignen und nicht selten kam es im Zuge von praktischen Tests zu unerwarteten Ergebnissen, die oft nur nach umfangreichen Analysen mit Hilfe des Systemteams oder des Zentralen Informatikdienstes der Universität Wien geklärt werden konnten.

\section{»Ein ideales Bibliothekssystem verzeiht Tippfehler bei der Suche«}

Im Falle eines falsch eingetippten Begriffs ganz im Stil von diversen etablierten Internet-Suchmaschinen mit »Meinten Sie ...« auf den Fehler aufmerksam gemacht zu werden, könnte in der Tat von einigen Bearbeiterinnen und Bearbeitern als eine Bereicherung empfunden werden, welche Alma aber derzeit nicht beinhaltet. Dem Projektteam machten während der Projektphase weniger die eigenen Tippfehler zu schaffen, als vielmehr die Übersetzungsfehler, die von Ex Libris in der deutschsprachigen Version des Bibliothekssystems vermehrt übersehen wurden. Sie sorgten oft für Verwirrung und stellen selbst heute noch Probleme dar, sodass gerne auch einmal in diversen Mailinglisten über irreführende Benennungen diskutiert wird. Auch die Tatsache, dass als Kommunikationssprache mit Ex Libris in erster Linie Englisch zum Einsatz kam, führte mehrfach zu Missverständnissen, wie etwa bei der Erläuterung diverser Arbeitsabläufe, die sich nicht immer reibungslos 1:1 übersetzen ließen. Ganz im Gegensatz dazu hat die Kommunikation und Zusammenarbeit mit den anderen österreichischen Bibliotheken ebenso wie mit der Verbundzentrale (OBVSG) sehr gut funktioniert und für alle teilnehmenden Institutionen eine unbestreitbare Bereicherung dargestellt. Neben der gemeinsamen Erstellung der Schulungsunterlagen konnten in Workshops und regelmäßigen Webkonferenzen Erfahrungen ausgetauscht und gemeinsame Problemlösungen erarbeitet werden. 


\section{»Ein ideales Bibliothekssystem ändert sich bis zu meiner Pensionierung nicht mehr allzu sehr«}

Dass Alma diesen Wunsch nicht erfüllen kann, musste die Verfasserin oder der Verfasser bereits wenige Wochen nach Abschluss der Datenmigration realisieren. Kurze Zeit nach dem Bearbeitungsstart in Alma an der Universitätsbibliothek Wien wurde nämlich von Ex Libris bereits ein neues User Interface implementiert, welches zu Beginn noch optional angewendet werden konnte, mit Jänner 2018 aber die ursprüngliche Benutzeroberfläche endgültig ablöste. Des Weiteren werden monatlich Updates in Alma eingespielt, die die Arbeitsabläufe der Bearbeiterinnen und Bearbeiter ebenfalls beeinflussen können. Diese sogenannten Releases umfassen in der Regel neue Funktionalitäten in unterschiedlichem Ausmaß, genauso wie Korrekturen von Fehlern im System. Änderungen müssen also keineswegs automatisch etwas Negatives bedeuten, sondern können durchaus auch eine Arbeitserleichterung mit sich bringen. Jedoch steht außer Frage, dass ein derart dynamisches Bibliothekssystem wie Alma von den Bearbeiterinnen und Bearbeitern enorme Flexibilität verlangt. Über diesen Umstand war sich auch die Direktion der Universitätsbibliothek Wien bewusst, weshalb sie die permanente Arbeitsgruppe Alma ins Leben gerufen hat. Es wurden Hauptverantwortliche und Stellvertreterinnen bzw. Stellvertreter aus den Bereichen Bibliothekssysteme, Benutzung, Formalerschließung, Sacherschließung, Benutzerdatenkoordination, Erwerbung, Zeitschriftenverwaltung, E-Ressourcen, Öffentlichkeitsarbeit, Helpdesk und Infodienst ernannt, deren Aufgabe es ist, sich mit den aktuellen Problemen in Alma auseinanderzusetzen und die für die Universitätsbibliothek Wien relevanten Releases $\mathrm{zu}$ testen und gegebenenfalls entsprechend an die Kolleginnen und Kollegen weiter zu kommunizieren. Dabei erhält die AG Alma Unterstützung von ehemaligen Mitgliedern des Projektteams und weiteren hausinternen wie auch verbundweiten Arbeits- und Expertinnen- bzw. Expertengruppen, da einige AGMitglieder nach wie vor die durch das Projekt entstandene interinstitutionelle Zusammenarbeit pflegen und die Kommunikation aufrechterhalten.

Auch in der AG Alma wird immer wieder deutlich, wie unterschiedlich die Erwartungshaltungen an ein Bibliothekssystem sein können. Verglichen mit den Rückmeldungen aus dem Kollegium im Zuge der Informationsveranstaltungen besteht eine relative Einigkeit im Hinblick auf die Übersichtlichkeit und die gegebenen Funktionalitäten: Alle bisherigen Tätigkeiten der Bibliothek sollten auch weiterhin umsetzbar sein und um jene erweitert werden, deren Verwaltung bislang nicht im System möglich war. Dabei sollen die Funktionalitäten klar strukturiert und einfach aufzufinden sein. Ob Alma, oder ein anderes System, diese Anforderungen erfüllt, liegt am Ende wieder im Auge der Betrachterin bzw. 
des Betrachters. Gerade deshalb ist es wichtig, den Veränderungen des Systems die entsprechende Aufmerksamkeit zu schenken. Nur wenn neue Funktionalitäten wahrgenommen werden und die Bibliotheken fortwährend durch ihr Feedback die Weiterentwicklung am Laufen halten, kann Alma sich zu einem System entwickeln, welches zwar nie für alle »ideal« sein, dafür aber immer mehr individuelle Anforderungen erfüllen wird. 


\section{Alle Zweigstellen unter einem (Koordinations-)Hut. Permanente Arbeitsgruppe Benutzung}

Als in den frühen 2000er Jahren immer mehr Fachbereichsbibliotheken an der Universitätsbibliothek Wien mit der Bibliothekssoftware Aleph der Firma Ex Libris zu arbeiten begannen, zeigte sich schnell, dass eine Koordination zwischen den einzelnen Bibliotheken unabdingbar war - die Arbeitsgruppe Entlehnkoordination war geboren.

Die Arbeitsgruppe setzte sich aus Mitarbeiter_innen verschiedener Fachbereichsbibliotheken und der Hauptbibliothek zusammen. Dabei wurde auf eine gute Durchmischung von großen und kleinen Zweigstellen sowie von Entlehnund Präsenzbibliotheken Wert gelegt.

Die Arbeitsgruppe beschäftigte sich zunächst mit der Definition von Regeln und Toleranzbestimmungen für die Ausleihe und die Verlängerung von Werken, damit an allen Zweigstellen bei der Gewährung von Ausnahmen, wie etwa der Verlängerung von überfälligen, jedoch nicht vorgemerkten Exemplaren, einheitlich vorgegangen wird. Dadurch wurde das von den Benutzer_innen häufig vorgebrachte Argument »aber das letzte Mal ging das an der Bibliothek XY schon« entkräftet.

Die Verwaltung von Handapparaten für Lehrveranstaltungen, Projekte und einzelne Handapparate der Forscher_innen beschäftigte die Arbeitsgruppe in all den Jahren immer wieder, da die erarbeiteten Lösungen häufig weiter angepasst werden mussten. Das damalige Ziel war es, jede Benutzung außer Haus als Ausleihe abzuwickeln. Mittlerweile werden die Lehrveranstaltungsapparate als Standortänderung innerhalb der Universitätsbibliothek behandelt. Die Forschungsapparate werden entweder als eigener Standort geführt oder weiterhin als Ausleihe gehandhabt, um das Bewusstsein dafür zu fördern, dass die Bücher Bibliothekseigentum sind. Literatur, die für Projekte benötigt wird, kann seit der Einführung des neuen Bibliothekssystems mit besonderen Benutzungsbedingungen entlehnt oder wie ein Forschungsapparat behandelt werden.

Im Zuge ihrer Arbeit stellte die Arbeitsgruppe fest, dass die Leihfristen für die Benutzer_innen in den einzelnen Fachbereichsbibliotheken einigermaßen unterschiedlich waren. So konnte man in der einen Fachbereichsbibliothek als 
Diplomand_in Bücher für 14 Tage entlehnen, in einer anderen für 28 und in einer dritten für 56 Tage, obwohl für alle drei Zweigstellen die Entlehndauer mit 14 Tagen angegeben war. Da sich selbst eingefleischte Bibliothekar_innen nicht merken konnten, wer an welcher Bibliothek wie viele Bücher wie lange ausleihen durfte, setzte man sich die Angleichung der Ausleihfristen zum Ziel. Es gab sogar die Idee einer einzigen Leihfrist für alle Zweigstellen.

In einem ersten Schritt wurden daher die Leihfristen für alle Benutzer_innengruppen in allen Zweigstellen analysiert, um so Gemeinsamkeiten herauszufinden. Schnell stellte sich jedoch heraus, dass die Ausgangsituation der einzelnen Bibliotheken sehr unterschiedlich ist und dass nicht die Leihfristen alleine, sondern auch andere Rahmenbedingungen - Verlängerungsmöglichkeiten, Mahnungen etc. - Einfluss auf die Benutzung der Bestände haben. Darüber hinaus wurde klar, dass selbst Präsenzbibliotheken Bücher regelmäßig zur Entlehnung außer Haus geben - über das Wochenende für Studierende, sehr viel länger für das Personal. Mit der großen Schwierigkeit, dass diese Titel von niemandem vorgemerkt werden können, wenn sie außer Haus sind, da sie als Präsenzexemplare definiert sind.

Im Rahmen von Gruppenarbeiten, bei denen Vertreter_innen aller 40 Zweigstellen teilnahmen, wurden die Bedenken und Bedürfnisse diskutiert, die schließlich als Grundlage eines Maßnahmenpaketes dienten. Es sollte für die gesamte Dienstleistungseinrichtung einheitliche, einfache und transparente Entlehnkonditionen differenziert nach Beständen und Benutzer_innengruppen geben:

einheitlich: für die gesamte Dienstleistungseinrichtung einfach: in der Erklärbarkeit und Merkbarkeit sowie in der Systemadministration transparent: eine Terminologie, die die tatsächlichen Verhältnisse abbildet differenziert: nach Beständen (entlehnbar oder nicht entlehnbar; wenn entlehnbar, dann maximal drei verschiedene Befristungen) und Benutzergruppen (händische oder automatische Verlängerung, Mahnung überfälliger Werke mit oder ohne Gebühren)

Vereinbart wurde eine Maximalentlehndauer von zwölf Monaten für alle entlehnbaren Bestände mit Ausnahme von Tagesentlehnungen. Die Erinnerungsschreiben für eine bevorstehende Rückgabe sollten sowohl eine Kennzeichnung von bereits vorgemerkten Werken als auch einen Hinweis und einen Link auf die Online-Verlängerungsmöglichkeit durch die Benutzer_innen selbst enthalten. Die Prüfroutinen für die Verlängerungen wurden so eingestellt, dass ausschließlich gemahnte und vorgemerkte Werke nicht mehr verlängert werden. Dies hatte zur Folge, dass noch nicht fällige, nicht vorgemerkte Werke verlängert werden konnten, obwohl es überfällige Werke auf dem Bibliothekskonto gab.

Da dieses Paket zu umfangreich war, um es auf einmal für die gesamte Universitätsbibliothek umzusetzen, wurde es in drei Pilotbibliotheken - der Fach- 
bereichsbibliothek Anglistik und Amerikanistik, der Fachbereichsbibliothek Publizistik- und Kommunikationswissenschaft und Informatik sowie der Fachbereichsbibliothek Theater-, Film- und Medienwissenschaft - mit umfangreichen Begleit- und Kommunikationsmaßnahmen getestet. Alle drei Bibliotheken stellten von einer Wochenend-Entlehnung auf eine 7-Tage-Entlehnung um. Aufgrund der überaus positiven Rückmeldungen seitens der Studierenden und des wissenschaftlichen Personals sowie der Mitarbeiter_innen der Fachbereichsbibliotheken stellten in den Jahren 2011 und 2012 mehrere »Präsenz«-Bibliotheken auf eine 7-Tage-Entlehnung um. Heute sind es nur mehr vier von ursprünglich 20 Fachbereichsbibliotheken, deren Bücher Nutzer_innen (mit Ausnahme des wissenschaftlichen Personals der Universität Wien) nur von Freitag bis Montag entlehnen dürfen.

Die Analyse von und Beschäftigung mit den Entlehnbedingungen führte innerhalb der Arbeitsgruppe zu einem tieferen Verständnis der Ausleihmechanismen einer Bibliothek. Das Zusammenspiel von Ausleihfrist, Vormerkungen und Verlängerungen stellte sich zudem komplexer dar als erwartet. Eine einzige Leihfrist für die gesamte Bibliothek blieb jedoch ein unerreichbares Ziel.

Nach diesem nicht ganz einfachen Projekt wandte sich die Arbeitsgruppe für kurze Zeit wieder vermeintlich leichteren Problemen zu, wie etwa der Definition einer Liste mit assoziierten Institutionen und der Überarbeitung oder genauer gesagt der Reduktion der Anzahl von Benutzer_innengruppen. Zeitgleich gab es das Projekt LIBRA, bei dem es um den »Laufenden Import und die Regelmäßige Aktualisierung von BenutzerInnendaten« aus den Systemen der Universitätsverwaltung ging, welches die AG Entlehnkoordination tatkräftig unterstützte. Aus diesem Projekt ging wiederum der Benutzerdatensupport hervor, der sich um die Dublettenbereinigung von Datensätzen und Sonderfälle kümmert und dem Projekt LIBRA beratend zur Seite stand.

Als 2015 die Entscheidung für das neue Bibliothekssystem Alma, erneut ein Produkt der Firma Ex Libris, getroffen wurde, erwuchsen der AG Entlehnkoordination neue Aufgaben. Die Arbeitsgruppe wurde während der Vorbereitungszeit bis zum Umstieg auf das neue System deutlich größer und umfasste zeitweilig mehr als 20 Mitglieder. Es mussten für sämtliche Benutzungsbereiche die Geschäftsprozesse festgehalten und Schnittstellen zwischen den Abteilungen beschrieben werden. Während der Implementierungsphase galt es, das System kennenzulernen, jede einzelne Funktion in Alma auf Herz und Nieren zu prüfen und das neue System an bestehende Workflows anzupassen bzw. umgekehrt. Dazu kamen die Erstellung von Schulungsunterlagen in Zusammenarbeit mit anderen österreichischen Bibliotheken, die ebenfalls auf Alma wechselten, und die Schulung von über 300 Mitarbeiter_innen der Universitätsbibliothek Wien in den Bereichen Ausleihe, Vormerkungen, Gebühren und Benutzer_innenverwaltung. Dabei durfte auch die Kommunikation mit den Benutzer_innen nicht 
zu kurz kommen, die vom Wechsel des Bibliothekssystems möglichst wenig spüren sollten.

Im Laufe dieser Vorbereitungen wurde aus der »AG Entlehnkoordination « die "AG Benutzung«. Die AG Benutzung ist heute eine ständige Arbeitsgruppe, die sich mit der bibliotheksweiten Koordination von Fragen aus den Bereichen Entlehnung, Alma-Fulfillment (z. B. benutzungsbezogene Workflows in Alma), Benutzer_innen-Datenverwaltung und Support, u:account, Benutzer_innengruppen, Online-Registrierung, Mahnungen und Gebühren sowie Benutzungsinformationen in der Suchmaschine u:search (Primo) beschäftigt. Die Arbeitsgruppe setzt sich mit diesen Fragen auseinander und unterbreitet der Direktion ihre Vorschläge und Ergebnisse. Dazu gehört auch die Kommunikation dieser Themen innerhalb der Bibliothek und die Schnittstelle zur Öffentlichkeitsarbeit.

Die Kolleg_innen des Benutzer_innen-Datensupports, die sich seit der Implementierung von LIBRA um den reibungslosen Ablauf der damit verbundenen Prozesse kümmern, sind inzwischen Teil der AG Benutzung. Sie betreuen die entsprechenden Anfragen in einem Ticketsystem und nehmen weitere Aufgaben im Bereich Benutzerdaten wahr (z. B. die regelmäßig stattfindenden Datenbereinigungen).

Die besondere Stärke der AG Benutzung liegt in den wechselnden Mitgliedern aus mittlerweile beinahe allen Zweigstellen, die aufgrund ihrer Teilnahme nicht nur ein vertieftes Wissen über das Bibliothekssystem erlangen, sondern auch die Gepflogenheiten und Probleme in anderen Zweigstellen kennenlernen. Durch den diesbezüglichen Gedankenaustausch finden sie oft auch Lösungen für die eigene Bibliothek und die gesamte Universitätsbibliothek. 


\section{Die Universitätsbibliothek als Informationskompetenzzentrum der Universität. Permanente Arbeitsgruppe Teaching Library}

\section{Organisatorisches}

Im Jänner 2013 wurde die Arbeitsgruppe (AG) Teaching Library als Ansprechgremium für alle Fragen der Teaching Library eingesetzt. Sie wurde mit der Erstellung und Umsetzung eines effizienten Konzepts, der Koordinierung der Entwicklung der Teaching Library und der Mitarbeit bzw. der Arbeit an zwei ebenfalls 2013 begonnenen Projekten beauftragt, die unter dem Punkt Projekte kurz vorgestellt werden. Seit 2018 wird sie im Organigramm als permanente Arbeitsgruppe geführt.

\section{Mitglieder}

Die Mitglieder der Arbeitsgruppe sind Michaela Zemanek (bis Ende Februar 2019 Leiterin der Fachbereichsbibliothek Psychologie), Manuela Rohrmoser (Leiterin der Abteilung ubw:helpdesk und User Training) und Karin Lach (Leiterin der Fachbereichsbibliothek Anglistik und Amerikanistik; organisatorische Leitung der AG Teaching Library). Alle drei Personen erfüllen auch außerhalb der Arbeit in der Arbeitsgruppe Aufgaben als Teaching Librarians in ihrem hauptsächlichen Arbeitsumfeld und sind Mitglieder (Rohrmoser, Lach) bzw. die Vorsitzende (Zemanek) der Kommission für Informationskompetenz der Vereinigung Österreichischer Bibliothekarinnen und Bibliothekare (VÖB). 


\section{Definitionen}

Im bibliothekarischen Sprachgebrauch des deutschsprachigen Raums versteht man unter dem Begriff Teaching Library »sämtliche Aktivitäten der Öffentlichen und Wissenschaftlichen Bibliotheken auf dem Gebiet der Förderung von Informations- und Medienkompetenz. « ${ }^{1}$

Informationskompetenz »bildet die Basis lebenslangen Lernens. [Sie] ermöglicht Menschen in allen Bereichen der Gesellschaft, Informationen zu suchen, zu bewerten, zu nutzen und effektiv zu erstellen, um ihre persönlichen, sozialen, beruflichen und bildungsmäßigen Ziele zu erreichen. ${ }^{2}$

\section{Arbeitsbereiche}

Im Rahmen der Tätigkeit der AG Teaching Library werden folgende Arbeitsbereiche abgedeckt:

- Unterstützende Mitarbeit bei der Entwicklung der Universitätsbibliothek als Teaching Library bzw. als Informationskompetenzzentrum der Universität Wien. Dabei ist es nicht wichtig, diese in der Bibliothekswelt gebräuchlichen Begriffe bekannt zu machen, sondern Benutzer_innen und Stakeholder bei der Erreichung ihrer informationsbezogenen Aufgaben und Ziele zu unterstützen. 2017 wurde z. B. in Zusammenarbeit mit dem Team Öffentlichkeitsarbeit eine Postkarten- und Lesezeichenaktion durchgeführt. Mit den Postkarten und Lesezeichen wurden in kurzen, prägnanten Sprüchen auf den prozesshaften Charakter des wissenschaftlichen Recherchierens bzw. auf mögliche Probleme von Studierenden beim Finden und Verwenden wissenschaftlicher Informationen und gleichzeitig auf Services der Universitätsbibliothek, die dabei helfen können, hingewiesen, insbesondere auf den Basiskurs Informationskompetenz auf der Lernplattform Moodle und das Schulungsangebot der Bibliothek.

- Die Mitglieder der Arbeitsgruppe stehen allen Kolleg_innen der Universitätsbibliothek Wien als Ansprechpartnerinnen in Sachen Teaching Library zur Verfügung. In diesem Rahmen erscheinen regelmäßig Berichte und eine Kolumne im Internen Newsletter der Universitätsbibliothek. Diese Kolumne dient dazu, Themenstellungen der Didaktik und Informationskompetenz bekannt zu machen, und lief bis Anfang 2019 unter dem Titel Teaching Tipp.

1 Sühl-Strohmenger, Wilfried: Teaching Library 2018. Siehe: https://bibliotheksportal.de/res sourcen/bildung/teaching-library/ [Letzter Zugriff: 11.09.2019].

2 IFLA: Leuchtfeuer der Informationsgesellschaft: Die Alexandria-Proklamation zu Informationskompetenz und lebenslangem Lernen (2015). Siehe: https://www.ifla.org/DE/publicati ons/node/8952 [Letzter Zugriff: 14.01.2019]. 
- Thematisch relevante Fort- und Weiterbildung und Vernetzung über die eigene Bibliothek hinaus:

- Organisation und Durchführung österreichischer TeachMeets, bei denen sich österreichische Bibliothekar_innen zu Themen der Informationskompetenz fortbilden und sich untereinander und, je nach Themenschwerpunkt, auch mit anderen Berufsgruppen wie Universitätslehrenden oder Lehrer_innen austauschen und vernetzen. (3. Österreichisches TeachMeet 2017 zum Thema Den informationskompetenten Umgang mit wissenschaftlicher Information Lehren/Lernen, 4. Österreichisches TeachMeet 2018 zum Thema Informationskompetenz und Schule.)

- Teilnahme, Netzwerken, Präsentation oder Programmgestaltung im Themenbereich Informationskompetenz bei nationalen und internationalen Bibliothekartagen und Informationskompetenzkonferenzen. Durch die Teilnahme zumindest eines Mitglieds der Arbeitsgruppe an der European Conference on Information Literacy (ECIL) seit ihrem Bestehen 2012 ist die Universitätsbibliothek Wien in der internationalen Informationskompetenzwelt bestens vernetzt. Besonders hervorzuheben sind die ersten beiden Informationskompetenz-Tage im deutschsprachigen Raum (2017 in Innsbruck und 2018 in Bamberg), die unter der Federführung von Michaela Zemanek ins Leben gerufen und gestaltet wurden.

\section{Projekte}

Folgende Projekte wurden bzw. werden gemeinsam mit anderen Mitarbeiter_innen der Universitätsbibliothek Wien durchgeführt:

- Erstellung eines Konzepts für die Durchführung von Informationskompetenzschulungen für Schüler_innen (abgeschlossen)

- Erstellung eines Kurses für Mitarbeiter_innen zur Bibliothekssuchmaschine $\mathrm{u}$ :search in Moodle (abgeschlossen)

- Erstellung, Wartung und Aufbau eines Basiskurses Informationskompetenz für Studierende der Universität Wien in der Lernplattform Moodle (1. Phase abgeschlossen, Wartung und Ausbau um weitere Pakete, Konzept für fachspezifische Kurse in Arbeit)

\section{Ausblick}

In nächster Zeit wird die Arbeitsgruppe ihr besonderes Augenmerk auf die Konzeptarbeit und die weitere Unterstützung anderer Bereiche der Bibliothek bei der Umsetzung der strategischen Ziele der Universitätsbibliothek, die die 
Themenbereiche Informationskompetenz und Teaching Library betreffen, legen. Der Basiskurs Informationskompetenz wird erweitert. Möglichkeiten, die Lernplattform Moodle oder andere Formate für die fachspezifische Informationskompetenz zu nutzen, werden analysiert. Ziel ist es, das entsprechende Angebot auszubauen, um Benutzer_innen beim Finden und Nutzen von fachlichen Informationsressourcen zu unterstützen. Fortgeführt wird die nationale und internationale Vernetzung im Rahmen der VÖB-Kommission Informationskompetenz sowie bei Kongressen und Tagungen. 


\section{Archiv}

Open-Access-Publikation im Sinne der CC-Lizenz BY-NC-ND 4.0

(c) 2019, Vandenhoeck \& Ruprecht GmbH \& Co. KG, Göttingen 
Open-Access-Publikation im Sinne der CC-Lizenz BY-NC-ND 4.0

(c) 2019, Vandenhoeck \& Ruprecht GmbH \& Co. KG, Göttingen ISBN Print: 9783847110989 - ISBN E-Lib: 9783737010986 


\section{Denn was zusammen gehört, findet auch zusammen? Das Universitätsarchiv als Teil der $\gg$ Dienstleistungseinrichtung Bibliotheks- und Archivwesen «}

Das Universitätsgesetz 2002 schuf für die österreichischen Universitäten grundlegend neue Voraussetzungen. Mit der Vollrechtsfähigkeit wurden die Universitäten erstmals seit dem Mittelalter wieder wahrhaft autonom, und sogar in der Gestaltung ihrer organisatorischen Binnenstrukturen weitgehend frei von gesetzlichen Detailregelungen.

Im Vorgängergesetz, dem Universitätsorganisationsgesetz (UOG) 1993, sah dies noch anders aus. So war sogar die Führung eigener Archive den Universitäten dank erfolgreicher "Lobbyarbeit" engagierter Archivarinnen und Archivare gesetzlich vorgeschrieben worden. Sie sollten im Rahmen der Dienstleistungseinrichtung "Zentrale Verwaltung" organisatorisch ihren Platz finden.

An der Universität Wien waren die Voraussetzungen diesbezüglich deutlich besser als anderswo. Seit 1874 wird das Universitätsarchiv durch historisch und archivwissenschaftlich geschulte Archivare betreut. Institutionell war es immer direkt unterhalb der Universitätsleitung angesiedelt, zunächst bei Rektorat und Akademischem Senat, ab dem UOG 1975 als Abteilung der Universitätsdirektion. Die Umsetzung des UOG 1993 erfolgte an der Universität Wien erst mit Beginn des Jahres 2000. Das Ergebnis war eine Satzung, in der, gewissermaßen "contra legem«, das Archiv der Universität Wien sogar als eigenständige Dienstleistungseinrichtung (DLE) aus der Zentralen Verwaltung herausgelöst wurde.

Als nur wenige Jahre später das Universitätsgesetz 2002 das Universitätsorganisationsgesetz 1993 ablöste, entfiel die gesetzliche Verpflichtung für die Universitäten, eigene Archive zu führen. In welcher organisatorischen Form würde das Universitätsarchiv weiter existieren? Die Antwort darauf lag nunmehr im autonomen Verfügungsbereich der Universitätsleitung. Es ist kein Geheimnis, dass die getroffene Entscheidung nicht den Vorstellungen der damaligen Archivleitung entsprach: Das Archiv wurde 2004 mit der Universitätsbibliothek zu der neu geschaffenen DLE Bibliotheks- und Archivwesen zusammengefasst. Die konkreten Motive dafür waren wohl das Vorbild einiger ausländischer Universitäten, sowie erhoffte, nicht zuletzt ökonomische »Synergie«-Effekte. An 
dieser Stelle ließen sich zahlreiche Argumentationslinien nachzeichnen, welche gegen, teilweise aber auch für die Vereinigung von Bibliotheken und Archiven sprechen. Im Augenblick der Verkündung des neuen Organisationsplanes herrschte jedenfalls im Archiv der Eindruck einer "feindlichen Übernahme" vor, auch wenn die Universitätsbibliothek selbst dafür gar nicht verantwortlich war.

Die Sterne für ein gedeihliches Miteinander schienen somit zunächst nicht besonders günstig zu stehen. Der gewissermaßen »natürliche« Reflex des Archivs in dieser verordneten Zweckgemeinschaft war die Betonung jener Eigenschaften, welche Archive und Bibliotheken voneinander unterscheiden: Dass Archive für Verwaltungsakten bzw. für in der aktuellen Archivwissenschaft gerne als "Records" bezeichnete "Verzeichnungseinheiten" mit Unikat-Charakter (im Gegensatz zu Büchern) zuständig sind; dass dafür unterschiedliche, archivkonforme Metadatenstandards zur Anwendung kommen; dass Archive keinen Sammlungsauftrag, sondern eine administrative Zuständigkeit haben; dass das Universitätsarchiv eine lange Tradition eigener Forschungs- und Editionstätigkeit aufweist; und noch einiges mehr.

War es diese Selbstbehauptungsstrategie, oder das von vornherein gegebene Verständnis der DLE-Leitung für die Sinnhaftigkeit archivgerechter Lösungen, welche das Zusammenleben Schritt für Schritt positiv beeinflussten? Auch die allgemeine Entwicklung der letzten Jahre hat wohl das ihre dazu beigetragen. Zwar sind nicht alle grundsätzlichen Einwände gegen die Vereinigung von Archiven und Bibliotheken ausgeräumt, doch sowohl die Rückbesinnung auf gemeinsame historische Wurzeln, als auch die jüngsten Entwicklungen im digitalen Bereich, deren Herausforderungen beide Einrichtungen gleichermaßen betreffen und auf eine noch kaum absehbare Weise verändern werden, führten dazu, dass einst recht starr gedachte Grenzen immer mehr aufweichen. ${ }^{1}$

Zum Auftakt der verordneten Zusammenarbeit galt es für das Archiv eine Errungenschaft zu bewahren, welche aus der kurzen Zeitspanne organisatorischer Eigenständigkeit herrührte: 2002/03 hatte es - als eines der ersten Archive Österreichs überhaupt - ein »Archivinformationssystem « zur elektronischen Verzeichnung von Archivbeständen implementiert. Bestand nun nicht die Gefahr, mit dieser Arbeit in das Prokrustesbett des Bibliothekssystems gezwungen zu werden? Die Befürchtung erwies sich als unbegründet. Nicht nur das: Als 2006 die Entscheidung anstand, die im Archivinformationssystem erfassten Daten mittels Online-Recherche zugänglich zu machen, wurde dies von der DLE-Leitung ausdrücklich gefördert und die Administration dem für die Universitätsbibliothek zuständigen Kolleg_innen des Zentralen Informatikdienstes (ZID) anvertraut.

1 Siehe dazu jüngst Christian Keitel: Zwölf Wege ins Archiv. Umrisse einer offenen und praktischen Archivwissenschaft. Stuttgart: Franz Steiner 2018, S. 74-76. 
Die Eingliederung in die neu geschaffene Dienstleistungseinrichtung hatte zwangsläufig die Mediatisierung des Archivs von der Universitätsleitung, damit aber auch von den wichtigsten "aktenproduzierenden « Stellen der Universität, zur Folge. Darüber hinaus führten die organisatorischen Umwälzungen $2004 \mathrm{zu}$ ungeplanten "Spontanablieferungen« größerer Aktenbestände. Diese Gegebenheiten machten die Formalisierung und Normierung von Archivierungsabläufen noch dringender erforderlich als zuvor. Deshalb wurde die Erarbeitung einer universitätsweiten Archivierungsrichtlinie in Angriff genommen, was auf Ebene der DLE-Leitung ebenfalls Unterstützung erfuhr. Nach eingehenden Beratungen und Präsentationen vor inneruniversitären "Stakeholdern" trat die Archivierungsrichtlinie der Universität Wien schließlich zu Beginn des Jahres 2011 in Kraft. Nur kurz davor war auch eine aktualisierte Archiv-Benutzungsordnung publiziert worden.

Besonders gefordert war das Universitätsarchiv durch die Mitwirkung an den Aktivitäten zum 650. Universitätsjubiläum 2015, welche schon einige Jahre davor ihren Anfang genommen hatten. Nicht nur die gesteigerte Nutzung der Archivbestände durch universitäre Jubiläumsprojekte, sondern auch der unmittelbare Beitrag des Archivs schlugen hier zu Buche. Bereits im Vorfeld wurden mit maßgeblicher Beteiligung des Archivs Tagungen organisiert sowie mehrere Bände in der Schriftenreihe des Universitätsarchivs und Matrikeleditionen herausgegeben. ${ }^{2}$

Den zentralen Beitrag des Archivs zum Jubiläum bildete - in Kooperation mit dem "Forum Zeitgeschichte der Universität Wien « - die Gestaltung einer umfangreichen Website zur Geschichte der Universität Wien, ein Projekt, welches von der DLE-Leitung von Anfang an finanziell und ideell gefördert wurde. ${ }^{3}$ Seine Fortführung als "work in progress« wurde in die Zielvereinbarungen aufgenommen.

Die Zeit nach dem Jubiläum brachte zunächst eine gewisse Konsolidierung der Archivarbeit mit der Konzentration auf Kernaufgaben. Umfangreiche Aktenablieferungen erforderten besondere Anstrengungen im Bereich der Erschließung (Ordnen und Verzeichnen). Dennoch hat das Archiv jüngst die Digitalisierung eigener Bestände intensiviert, wofür sich die DLE-interne Kooperation mit den Teams von PHAIDRA und »ubw:innovation« als sehr nützlich erwiesen hat. Auch wenn unter Archivar_innen hin und wieder über »Digitalisierungspopulismus« geklagt wird, so führt an der Bewältigung des »digital turn« auch in den Archiven kein Weg vorbei. Darin liegen zweifellos die größten

2 Siehe https://bibliothek.univie.ac.at/archiv/publikationen.html [Letzter Zugriff: 10.01.2019].

3 650plus - Geschichte der Universität Wien, https://geschichte.univie.ac.at/ [Letzter Zugriff: 10.01.2019]. 
Herausforderungen der unmittelbaren Zukunft - sowohl für Bibliotheken als auch für Archive. 


\section{Hauptbibliothek}


Open-Access-Publikation im Sinne der CC-Lizenz BY-NC-ND 4.0

(c) 2019, Vandenhoeck \& Ruprecht GmbH \& Co. KG, Göttingen ISBN Print: 9783847110989 - ISBN E-Lib: 9783737010986 


\section{There and Back Again ... ' Die Abteilung Alte und wertvolle Bestände}

Der historische Buchbestand an der Hauptbibliothek der Universitätsbibliothek Wien ist in seiner Zusammenstellung ein Zeugnis für deren Entwicklung von der Neugründung 1777 über den massiven Zuwachs im 18. Jahrhundert bis zum Aufstieg zu einer der bedeutendsten Bibliotheken im damaligen Habsburgerreich. Bis heute nutzen Forscher_innen aus dem In- und Ausland vor allem die Bestände aus der Spätzeit der Monarchie, die in vielen anderen Bibliotheken durch die Kriegswirren sowohl des Ersten als auch des Zweiten Weltkrieges verloren gingen.

\section{Bestandsübersicht}

Der Eröffnung der neugegründeten Universitätsbibliothek Wien im Mai 1777 ging eine kaiserliche Verfügung im Jahr 1773 voraus, die mit der Vereinigung dreier Jesuitenbibliotheken für einen Bestand von etwa 45.000 Bänden am Eröffnungstag sorgte. Für ein rasches Anwachsen des Bestandes sorgten zwei Umstände: Einerseits wurden unter Kaiser Josef II. zahlreiche Klöster aufgelöst, deren Bestände teilweise an die Universitätsbibliothek Wien gingen; andererseits erhielt die Universitätsbibliothek Wien bereits 1781 das Pflichtexemplarrecht, das regelmäßig erneuert wurde. ${ }^{2}$

Heute beinhaltet die Sammlung 652 Inkunabeln, deren älteste eindeutig datierbare Edition eine Ausgabe der Historia Naturalis ${ }^{3}$ von Plinius dem Älteren aus dem Jahr 1469 ist. Den Schwerpunkt der Inkunabelsammlung bilden theo-

1 In Anlehnung an den Roman Der Hobbit oder Hin und Zurück von J. R. R. Tolkien.

2 Nähere Informationen zur Geschichte der Universitätsbibliothek sind in den beiden Beiträgen von Nina Knieling sowie Markus Stumpf und Christina Köstner-Pemsel auf der Website zur Geschichte der Universität Wien zu finden: https://geschichte.univie.ac.at/de/themen/die-uni versitaetsbibliothek.

3 Volltext auf PHAIDRA: https://phaidra.univie.ac.at/o:19958. 
logische Werke, danach folgen Werke aus den Rechts- und Staatswissenschaften, der klassischen Philologie sowie der Philosophie und Geschichte. ${ }^{4}$

In den Raramagazinen der Hauptbibliothek werden mehr als 7.100 Titel aus dem 16. Jahrhundert, etwa 21.000 aus dem 17. Jahrhundert sowie etwa 52.000 aus dem 18. Jahrhundert verwahrt. Dazu kommen Manuskripte, aber auch der alte thematische Katalog der Universitätsbibliothek, das sogenannte Localrepertorium sowie historische Bibliothekskataloge. Leider ist aus der Zeit der alten Universitätsbibliothek nur mehr der Katalog der Versteigerung der Werke, die als Dubletten nicht in die damalige Hofbibliothek aufgenommen wurden, erhalten, jedoch kein Bestands- oder Übergabeverzeichnis aus 1756.

\section{Benutzung}

Einer Sisyphusarbeit gleicht eine der zentralen Aufgaben der Abteilung, nämlich die bibliothekarische Bearbeitung und buchwissenschaftliche Erschließung des umfangreichen vorliegenden Bestandes, der zwischen 1450 und 1780 erschienen ist. Die verstärkte systematische Katalogisierung der Bestände des 16. und 17. Jahrhunderts wurde unter der Leitung von Leopold Cornaro, der 2007 in Ruhestand ging, begonnen, musste aber in den darauffolgenden Jahren stark intensiviert werden. Hatte zu Beginn die Erfassung ausschließlich durch Mitarbeiter der Abteilung stattgefunden, so konnte in den letzten zehn Jahren mit intensiver Unterstützung der Abteilung Formalerschließung mittlerweile die Katalogisierung des Bestandes aus dem 15. und 16. Jahrhundert fast abgeschlossen werden. Aus dem 17. Jahrhundert ist auch etwa ein Drittel der Werke online auffindbar. Damit ist für die Benutzer_innen dieser Bestand wesentlich einfacher zugänglich, auch wenn für einen guten Teil des historischen Bestandes nach wie vor die früheren Rechercheinstrumente, der sogenannte »Kapselkatalog « sowie der Bandkatalog - der zwar digital, aber ohne Volltextsuche zur Verfügung steht -, zu Rate gezogen werden müssen. Um diese Bücher nicht mit einem Bestellzettel direkt vor Ort bestellen zu müssen, wurde zur leichteren Benutzung dieses nicht im Bibliothekssystem erfassten Bestandes die Möglichkeit geschaffen, diese Werke elektronisch in der Suchmaschine über die Funktion »Altbestandsbestellung« zu ordern.

Eine massive Verbesserung der weltweiten Zugänglichkeit, aber auch im Sinne des Bestandsschutzes, stellte der Beginn der Digitalisierung dar. Dank einer Anschubfinanzierung durch das Projekt EOD@Culture (dem ersten von der Europäischen Union geförderten Projekt, das an der Universitätsbibliothek

4 Ausführlich dazu: Hugo Alker: Universitätsbibliothek Wien - Katalog der Inkunabeln, 2., durchges. und erw. Aufl., hg. von Leopold Cornaro. Wien: Universitätsbibliothek Wien 1996. 
Wien durchgeführt wurde) konnten sowohl Knowhow aufgebaut als auch geeignete Buchscanner angeschafft werden. Im Rahmen des Projekts Europeana Libraries wurden mehr als 100 Inkunabeln digitalisiert und open access zur Verfügung gestellt. Seit 2008 werden konsequent Werke aus den Raramagazinen digitalisiert, mit Metadaten versehen und in PHAIDRA, dem Repositorium der Universität Wien, archiviert und veröffentlicht. Die digitalisierten Sammlungen sind damit sowohl über die Suchmaschine der Bibliothek als auch direkt im Repositorium $^{5}$ auffindbar.

\section{Rück- und Ausblick}

Im Jahr 2018 haben mehr als 2.000 Benutzer_innen fast 2.000 Öffnungsstunden im Lesesaal »Alte und wertvolle Bestände» verbracht, um jenen Bestand der Hauptbibliothek einzusehen und zu nutzen, der vor 1919 erschienen ist, sowie die Großformate. Geschätzt werden die ruhige, im Sommer kühle Atmosphäre, die kompetente Betreuung der Benutzer_innen im Lesesaal und die Möglichkeit, den vorhandenen Buchscanner zu nutzen - sofern es sich nicht gerade um Bücher aus dem Raramagazin handelt. Langjährige Mitarbeiterinnen und Mitarbeiter verwenden bis heute gelegentlich den Begriff »Sonderlesesaal«, wenn sie vom »Lesesaal Alte und wertvolle Bestände« sprechen, da der Lesesaal erst seit 2013 in einem neuen Erscheinungsbild wieder an seinen ursprünglichen Ort zurückgekehrt ist. In den Jahren zwischen 2007 und 2013 wechselte der Lesesaal in mehreren Etappen seinen Standort: im Jahr 2007 wurde der damalige Sonderlesesaal aufgelöst und mit dem Zeitschriften-Lesesaal zusammengelegt. Nach dessen Übersiedlung in die Teinfaltstraße fand der Lesesaal »Alte und wertvolle Bestände« eine interimistische Heimat auf Stiege IX, wo ein Besuch allerdings nur auf Anfrage möglich war. Danach konnte man den historischen Bestand einige Zeit im jetzigen Katalogsaal 3 einsehen, bevor der zwischenzeitlich als interner Besprechungsraum genutzte, ursprüngliche Sonderlesesaal unter dem neuen Namen »Lesesaal Alte und wertvolle Bestände« wieder zu seiner aktuellen Bestimmung fand.

Zusätzlich wickelt die Abteilung in Zusammenarbeit mit der Direktionsassistenz die Leihansuchen von Museen aus aller Welt ab, holt Schätzwerte ein, erstellt ein Schutzdigitalisat der jeweiligen Leihgabe, kümmert sich um die Einhaltung der konservatorischen Vorgaben und erhält als Dankeschön bei manchen Ausstellungen eine Kurator_innen-Führung.

5 Collection »Digitalisierte Bestände der Universitätsbibliothek Wien« siehe: https://phaidra. univie.ac.at/detail/o:73331. 
Eine erfreuliche Entwicklung stellt das rege Interesse der Lehrenden der Universität Wien, die regelmäßig mit ihren Studierenden zu uns kommen, an speziellen Einschulungen in die Nutzung des historischen Bestandes dar. Weiters empfangen wir immer wieder ausländische Gäste der Universität Wien, die dann eine "Special Limited Edition" erhalten: eine Führung nicht nur durch die Raramagazine, sondern auch durch die Geschichte der Universitätsbibliothek Wien. 


\section{Ein bisschen Werbung für die Erwerbung. Die Abteilung Bestellung und Erwerbung}

In jeder Bibliothek werden Medien erworben - so auch an der Universitätsbibliothek Wien. Doch was genau geschieht hier? Einfach nur kaufen und Rechnungen bezahlen? Nein, es ist doch etwas mehr.

Von den technischen Vorgaben durch die Bibliothekssysteme bis zu den rechtlichen Vorgaben aus dem Vergaberecht, dem Urheberrecht und der Datenschutzgrundverordnung: All das hat Einfluss auf die typischen Aufgaben, die sich zwar regelmäßig wiederholen, aber doch immer äußerst genau ausgeführt werden müssen.

In der Erwerbung beginnt der Weg unserer Medien zu unseren Benutzer_innen. Eine spannende Reise. Natürlich war es schon immer das oberste Ziel, den Weg besonders kurz und effizient zu gestalten, die Aufgaben der Abteilung haben sich im Laufe der Jahre aber doch stark gewandelt und erweitert. Da sind zum einen die Arten der Medien, die erworben werden, und zum anderen die Erwerbungsmodelle selbst. Hier haben sich in den letzten Jahren ganz neue Welten aufgetan.

Die Erwerbungspolitik der Universitätsbibliothek Wien hat sich eine ganz klare Präferenz zur elektronischen Version auf die Fahnen geschrieben und übernimmt hier oft eine Vorreiterrolle. Ziel ist ein möglichst breites Angebot mitunter nur auf Zeit; eine dauerhafte Archivierung ist nur für ausgewählte Titel vorgesehen. Erwerbungsmodelle wie z. B. »Evidence Based Acquisition" und Publikationsmodelle wie »Print-on-Demand« haben die Bibliothekslandschaft auch für die Benutzer_innen stark verändert.

Zwar wird auch heute noch der Bedeutung von gedruckten Büchern Rechnung getragen und diese werden auch immer noch von unseren Benutzer_innen angefragt, ihr Anteil sinkt allerdings stetig und so ist die Tendenz eindeutig: die elektronischen Versionen werden beim Ankauf bevorzugt. Das hat konkrete Auswirkungen auf den Workflow der Erwerbung in der Hauptbibliothek: Für jeden Bestellwunsch wird zunächst überprüft, ob der gewünschte Titel elektronisch verfügbar ist. Erst im negativen Fall wird der Bestellvorgang für die Anschaffung eines Printexemplars eingeleitet. Auch bei Reparaturen in der Ein- 
bandstelle wird vor der Beauftragung auf elektronische Verfügbarkeit geachtet. Diese Arbeitsschritte finden in enger Kooperation mit dem Zentralen Service EResource Management statt.

Nach diesen Abklärungen folgen die typischen Arbeitsschritte - die Kernkompetenzen der Abteilung: Bestellung, Etat- und Lieferant_innenverwaltung, Verrechnung, Inventarisierung und Statistik im Bibliothekssystem. Die Freigabe von Rechnungen entsprechend dem Vier-Augen-Prinzip erfolgt mittels eigenem Workflow in SAP.

Apropos Bibliothekssystem: Stetiger Wandel auch hier - vom eher einfach gestrickten BIBOS über Aleph zum doch recht komplexen Alma - es waren jedes Mal Quantensprünge in der Entwicklung. Was das 2017 eingeführte Bibliothekssystem Alma betrifft, war die Universitätsbibliothek Wien von Anfang an ganz vorne dabei und hat sich schon in der Ausschreibungsphase stark für Verbesserungen und Weiterentwicklungen engagiert. Der Aufwand und der erforderliche persönliche Einsatz in der Implementierungsphase waren eine große Herausforderung - nicht nur für die Abteilung Erwerbung. Auch nach dem »GoLive« von Alma müssen laufend Ressourcen für die monatlichen Updates, die mitunter sehr tiefgreifende Veränderungen mit sich bringen, abgestellt werden. Die Updates erfordern laufend Tests, Anpassungen der Workflows und Schulungen. In den österreichweit organisierten Anwendergruppen, aber auch in der internen "AG Alma" der Universitätsbibliothek, finden hierzu regelmäßig Treffen statt. Es haben sich hier Möglichkeiten für regen Austausch gebildet dieser Austausch ist für den Bereich Erwerbung als wirklich erfreulich zu werten.

Ein weiteres Betätigungsfeld der Abteilung stellt das Zentrale Management von Zeitschriftenrechnungen (ZZV) dar. Zeitschriftenrechnungen der gesamten Universitätsbibliothek Wien werden normiert erfasst bzw. kontrolliert. Ziel ist vor allem die Vollständigkeit des laufend gehaltenen Zeitschriftenbestandes und Kostenkontrolle im Bereich Zeitschriften.

Ein sehr wichtiges Handlungsfeld der Abteilung ist der Bereich Hochschulschriften. War es vor 20 Jahren ein eher einfaches Gebiet im Aufgabenportfolio der Erwerbung, hat sich hier ein sehr intensives Arbeitsfeld erschlossen, das auch sehr fruchtbare Kooperationen mit dem Zentralen Informatikdienst und dem Studien- und Lehrwesen - genauer dem Büro Studienpräses - gebracht hat. Hierher gehörte zunächst die Einrichtung eines Hochschulschriftenservers und der zugehörigen Services. Die Kooperation mit dem Studien- und Lehrwesen ergab aber zeitgleich das Erfordernis der Einrichtung eines Services zur Plagiatsprüfung eingereichter Hochschulschriften, die 2007 startete und 2008 in den Regelbetrieb ging. Das Service ist mittlerweile in der Universitätslandschaft bestens etabliert und umfasst folgende Vorgänge: Einreichung der elektronischen Version der Hochschulschrift / Überprüfung durch die Plagiatssoftware / Beurteilung und Freigabe / Übertragung an den Hochschulschriftenserver / 
Redaktion der Metadaten durch die Bibliothek / Verknüpfung des Katalogisats bzw. Erstellung eines Katalogisats im Bibliothekssystem / Verwaltung der Volltexte. Die Abteilung Erwerbung leistet den First-Level-Support telefonisch und per E-Mail für den gesamten Workflow, ist unter anderem bei der Erstellung von PDF-Dateien, Fragen zum Upload sowie der Volltextanzeige behilflich und verwaltet den Hochschulschriftenserver sowie die Print-Exemplare der Hochschulschriften. Natürlich mussten die technischen und rechtlichen Rahmenbedingungen innerhalb der letzten elf Jahre immer wieder adaptiert werden, zuletzt aufgrund der Datenschutzgrundverordnung. Hier ist im Support auch immer wieder intensive Beratung der angehenden Absolvent_innen nötig. Für die nähere Zukunft geplant ist eine Erneuerung und Darstellung des Hochschulschriftenservers als eigener Teilbereich des Repositoriums PHAIDRA. Da schon geringe Offline-Zeiten des Services sofort bemerkt werden und laufend Hochschulschriften von Absolvent_innen abgeschlossen werden, ist dieses Gebiet recht sensibel. Um lange »Stehzeiten« beim Neustart und der Datenmigration zu vermeiden, muss eine sorgfältige Planung erfolgen. Das Projekt befindet sich derzeit in Entwicklung in enger Kooperation mit dem Zentralen Informatikdienst.

Doch nicht nur Software und Erwerbungsmodelle verändern sich, 2011 wurde die Abteilung selbst im Zuge von Brandschutzmaßnahmen umgebaut und neu eingerichtet. Geringfügiger Platzverlust wird durch eine besonders gelungene, funktionale Ausstattung und Einrichtung mehr als wettgemacht.

Die weitreichenden Veränderungen und die vielen kleinen Herausforderungen des Alltags hat Maria Seissl stets mit viel Unterstützung und großem Verständnis begleitet, wofür ihr großer Dank gilt. 
Open-Access-Publikation im Sinne der CC-Lizenz BY-NC-ND 4.0

(c) 2019, Vandenhoeck \& Ruprecht GmbH \& Co. KG, Göttingen ISBN Print: 9783847110989 - ISBN E-Lib: 9783737010986 


\section{Die ELFe der Hauptbibliothek. Die Abteilungen Entlehnung und Lehrbuchsammlung und Fernleihe}

Neulich kam eine Leserin in die Entlehnung der Hauptbibliothek mit den Worten: »Die Rückgabe in der Entlehnung ist jetzt aber neu, oder?« Diese Frage musste - nicht ohne ein gewisses Schmunzeln - verneint werden, schließlich war die Rückgabe jahrzehntelang in der Entlehnung und sie ist es seit einigen Jahren wieder. Insgesamt hat sich in den letzten 15 Jahren viel getan.

\section{Räumliches}

Die Entlehnung ist zwar seit Mitte der 1960er Jahre in denselben Räumlichkeiten untergebracht, dennoch haben sich die Funktionszonen - Buchausgabe, Rückgabe, Ausweisverwaltung - zwischenzeitig mehrmals geändert. Gab es anfangs noch Multifunktionsschalter für die Buchausgabe, -rücknahme und das Gebühreninkasso, hat es sich im Laufe der Jahre durch die rasant gestiegene Anzahl der Benutzungsfälle Mitte der 2000er Jahre mit mehr als 500.000 Entlehnungen (und Rückgaben) pro Jahr herausgestellt, dass eine räumliche Trennung der Funktionen Buchausgabe und Rücknahme effektiver ist und zu kürzeren Wartezeiten führt.

Die Rückgabe wurde 2013 räumlich wieder der Entlehnung eingegliedert, dennoch blieb dieser Bereich weiterhin von der Buchausgabe funktionell getrennt. Ein Experiment mit Rückgabeklappen führte nicht zum gewünschten Erfolg. Die Benutzer_innen waren aufgrund der bei der Rückgabe verursachten Geräusche deutlich befremdet, da es wegen des Hohlraums im Auffangbehälter so klang, als würden die abgegebenen Bücher in ein tiefes Loch fallen und dadurch beschädigt werden. Trotz der Einführung von automatisch versendeten Rückgabequittungen fehlte den Benutzer_innen der persönliche Kontakt mit den Mitarbeiter_innen, um eventuelle Fragen und Bedürfnisse, wie etwa Verlängerungen oder sofortige Wiederausleihen, schnell abklären zu können. Bei den Mitarbeiter_innen wiederum führte die Lärmentwicklung bei der Betätigung der Klappen zu einer gewissen Belastung und in einigen Fällen zu Kopf- 
schmerzen. Da letzten Endes die Benutzer_innen, Mitarbeiter_innen und selbst das Material der Klappen ermüdet waren, wurden nach ca. eineinhalb Jahren wieder personenbesetzte Rückgabeschalter eingeführt.

Zeitgleich mit den Klappen wurde das Gebühreninkasso an einen Kassenautomaten im Foyer ausgelagert, sodass in der Abteilung die Handkassen und der Umgang mit Bargeld aufgegeben werden konnten. Eine Kassa liegt zwar für den Notfall bereit, erweckt aber eher nostalgische Gefühle, da sie in den letzten fünf Jahren nie benutzt werden musste.

Der Bereich für die Ausweisverwaltung wurde in einem ersten Schritt in einen eigenen Raum verlegt, da bei der Einschreibung viele personenbezogene Daten kommuniziert werden und so nicht jede bzw. jeder mithören kann.

Mit dem längst fälligen Umbau im Jahr 2012 - es war noch Möblierung aus den 1980er Jahren vorhanden - wurde der Ausweisschalter der Entlehnung mit dem Ausgabeschalter der Fernleihe zusammengelegt. Dadurch ergab sich ein Synergieeffekt, von dem beide Abteilungen profitieren. Die Mitarbeiter_innen am Ausweisschalter übernahmen die Ausgabe der bestellten Fernleihe-Literatur sowie das Kassieren der angefallenen Fernleihegebühren. Die Beratung und Information bei Fernleihebestellungen erfolgten weiterhin durch das Team der Fernleihe. Die Entlehnung gewann dadurch mehr Platz und kann die sensible Kommunikation bei der Einschreibung von Benutzer_innen in ruhiger Atmosphäre abwickeln, während die Mitarbeiter_innen der Fernleihe bei Bedarf in der Entlehnung aushelfen können.

\section{Funktionelles}

Bereits im Jahr 2007 wurde für den Ausdruck der Bestellungen aus dem Büchermagazin verschiedenfarbiges Papier eingeführt. Die Farbe wechselt mit jedem Tag, montags gibt es etwa gelbe Ausdrucke, dienstags grüne usw. Da nach Ablauf einer Woche die zwar bestellten, aber nicht abgeholten Werke wieder aus dem Bereitstellungsregal gezogen werden müssen, kann man sich einfach an der Farbe des Papiers orientieren und muss nicht das aufgedruckte Datum der Bereitstellungsfrist lesen.

Die Sortierung der Bereitstellungen wurde ebenso angepasst. Die Abteilung hat durchschnittlich 5.000 bis 8.000 Bereitstellungen im Regal zu verwalten. Täglich werden ca. 1.500 Bücher bestellt, entlehnt und ebenso viele zurückgegeben, sodass eine rege Fluktuation gegeben ist. Die Bestellungen wollen schnell und effizient sortiert, in die Regale geschlichtet und zur Buchausgabe wiedergefunden werden.

Die alte Sortierroutine sah vier Stufen vor: (1) Grobsortierung nach den Nachnamen A-L und $\mathrm{M}-\mathrm{Z}$ aufgrund der großen Menge an bestellten Büchern, 
(2) Sortierung nach dem Alphabet, wobei SCH und ST aufgrund der Menge als eigene »Buchstaben« betrachtet werden, (3) Sortierung zwischen Einzel- und Mehrfachbestellungen, und zuletzt (4) Feinsortierung von Mehrfachbestellungen nach den Namen der Benutzer_innen.

Die Lagerung der bestellten Bücher nach den Familiennamen nahm in den jeweiligen Regalen viel Platz ein und bei Buchstaben, die häufig am Beginn des Namens stehen, dauerte die Suche dementsprechend lang. Für den Fall, dass eine Benutzerin oder ein Benutzer an einem Tag fünf Bücher bestellte und an einem anderen nur eines, lagen die ersten fünf Werke im Namensstapel, das eine aber bei den Einzelbestellungen, sodass man oft an zwei Stellen suchen musste.

Die neue Sortierroutine behielt die Grobsortierung und die Sortierung nach dem Alphabet bei, jedoch wurde innerhalb eines Buchstabens nicht mehr nur nach dem Nachnamen sortiert, sondern auch nach dem zweiten Buchstaben (bzw. bei SCH und ST entsprechend nach dem vierten und dritten), also KA, KE, $\mathrm{KI}, \mathrm{KO}$ oder KU. Alle anderen Kombinationen - wie etwa KR(amer) und KL(inger) - wurden unter »K gemischt« zusammengefasst. Die Feinsortierung nach den Familiennamen wurde aufgegeben. Im laufenden Betrieb hat sich gezeigt, dass in den so entstandenen kleinteiligeren Bereichen rascher und zielgerichteter gesucht werden kann und somit die Abwicklung am Schalter beschleunigt wurde.

Anders als andere Magazinsbibliotheken in Österreich hatte die Hauptbibliothek immer festgelegte Aushebezeiten (im Gegensatz zu einer permanenten Aushebung) und somit im Sinne eines Service-Level-Agreements fixe Zeiten, ab denen die Bestellungen abgeholt werden konnten. Dies führte immer wieder zu Missverständnissen seitens der Benutzer_innen, die sich schwertaten, zwischen Bestellungen aus dem Magazin ohne damit verbundene Benachrichtigungen einerseits und Vormerkungen von entlehnten Werken mit Benachrichtigungen andererseits zu unterscheiden.

Mit der Auslagerung von Beständen in ein Depot am Stadtrand im Jahr 2016 wurde es zwingend notwendig, das Eintreffen der Bestellungen dieses Standortes im Abholregal im Bibliothekssystem zu quittieren, um sie bereitzustellen und automatisch eine Nachricht an die Benutzerin oder den Benutzer zu schicken. Da zu diesem Zeitpunkt bereits der Vertrag für die neue Bibliothekssoftware Alma der Firma Ex Libris abgeschlossen war und diese empfiehlt, alle Magazinsbestellungen bereitzustellen, wurde dies ab Herbst 2016 bibliotheksweit umgesetzt. Das führte zwar zu einem deutlichen Mehraufwand seitens der Bibliothek, da jedes einzelne Buch gescannt werden muss; jedoch zeigte sich, dass dadurch die Kommunikation mit den Benutzer_innen einfacher wurde, da man nun darauf hinweisen kann, die Benachrichtigung per E-Mail abzuwarten, anstatt wie bisher auf die verschiedenen Abholzeiten hinzuweisen. 
Die Einführung des neuen Bibliothekssystems Alma stellte auch die Fernleihe vor neue Herausforderungen: Die Geschäftsprozesse wurden an ein Cloudsystem angepasst sowie alle Briefe und Ausdrucke überarbeitet. Die Fernleihebestellungen werden derzeit über ein Mailformular an die Abteilung geschickt, von den Mitarbeiter_innen in Alma eingepflegt und dort weiterbearbeitet. Eine wesentliche Erleichterung im Betrieb der Fernleihe stellt die Einführung der internen Abrechnung der Bestellungen für das Personal der Universität Wien im Oktober 2018 dar. Dadurch muss das Personal der Universität Fernleihe-Rechnungen nicht mehr bezahlen und später bei der Dienstleistungseinrichtung Finanzwesen und Controlling einreichen, sondern es wird direkt zwischen den Dienstleistungseinrichtungen der Universität verrechnet.

Die nächste große Herausforderung für die Fernleihe wird die möglichst automatische Abwicklung der Fernleihebestellungen sein. Im Idealfall geben Benutzer_innen Bestellungen in u:search auf, diese werden automatisch den Lieferbibliotheken zugeordnet und versendet und dort aufgrund ebenso automatisch gedruckter Bestellscheine weiterbearbeitet. Besteller_innen werden über jeden einzelnen Schritt informiert und wissen über den Fortschritt der Bestellung Bescheid.

\section{Personelles}

In den 2000er Jahren gab es in der Entlehnung der Hauptbibliothek einen Höchststand an Benutzung, wodurch einige Jahre lang - wie oben erwähnt - über 500.000 Ausleihen pro Jahr zu verzeichnen waren (und natürlich ebenso viele Magazinsbestellungen und Rückgaben). Diesem gestiegenen Arbeitsaufwand wurde durch die Anstellung von zeitlich befristeten, geringfügig Beschäftigten Rechnung getragen. Wurden diese zuerst vorwiegend für die Abwicklung der Spätdienste eingesetzt, zeigte sich schnell auch tagsüber ein Bedarf an zusätzlichen Mitarbeiter_innen.

In der Abteilung gab es viele Jahre lang eine ständig gleichbleibende Diensteinteilung, wodurch manche Mitarbeiter_innen mehrere Jahre lang beinahe ausschließlich dieselben Tätigkeiten verrichteten. Dies führte zwar zu einer hohen Fachexpertise im eigenen Bereich, ging aber mit wenig Fachwissen in den anderen Bereichen der Abteilung einher. Insofern war bei Krankheitswellen oft zu wenig Flexibilität gegeben, weshalb 2012 ein Dienstrad eingeführt wurde. Dieses sieht vor, dass die Vollzeitmitarbeiter_innen jeden Tag der Woche an einer anderen "Station « eingeteilt sind, also einen Tag in der Rückgabe, am nächsten in der Buchausgabe, am dritten bei der Ausweisverwaltung usw. Nach anfänglichen Bedenken seitens der Mitarbeiter_innen zeigten sich schnell die Vorteile der neuen Regelung: Das Personal kann flexibler eingesetzt werden, man bleibt in 
allen Bereichen auf dem Laufenden, und auch die Zusammenarbeit, der Zusammenhalt und das Verständnis untereinander hat sich dadurch deutlich verbessert. Die geringfügig Beschäftigten, die oft nur wenige Tage der Woche in der Abteilung arbeiten, werden nach Möglichkeit ebenfalls in einer Art Rotation eingeteilt.

Durch das hohe Benutzungsaufkommen in der Abteilung wurde die Grundeinschulung neuer Mitarbeiter_innen im Bereich Ausleihe für den gesamten Bibliotheksbetrieb, also auch für die Fachbereichsbibliotheken, übernommen. Die zweistündige Kurzeinschulung sieht die Vermittlung von Basiswissen und -tätigkeiten direkt an den Schaltern vor, sodass neue Mitarbeiter_innen sofort praktische Erfahrung sammeln können. Die theoretische Einschulung für die Ausweisverwaltung findet ebenso in der Entlehnung statt, wo im Anschluss die praktische Anwendung am Ausweisschalter unter Anleitung einer Mitarbeiterin bzw. eines Mitarbeiters der Abteilung erfolgt. Die Schulungstätigkeit fördert die Kommunikation und das Verständnis zwischen den Fachbereichsbibliotheken und der Hauptbibliothek.

Als vor einigen Jahren Notfall-Springerdienste zur Aufrechterhaltung des Betriebes in den Fachbereichsbibliotheken eingeführt wurden, war die Teilnahme der Entlehnung an dieser Maßnahme fast ein logischer Schluss, da es durch die große Anzahl der hier beschäftigten Mitarbeiter_innen (durchschnittlich 15,25 Vollzeitäquivalente bzw. 30 Personen) oft leichter ist, eine Person abzustellen, als dies bei weniger Mitarbeiter_innen der Fall ist. Zudem profitieren die »Springer_innen« selbst davon, denn sie lernen andere Zweigstellen und deren Mitarbeiter_innen besser kennen.

Auch in der Fernleihe gab es personelle Veränderungen: Obwohl die Bestellungen aus bzw. die Lieferungen an andere Bibliotheken in den letzten 15 Jahren annähernd gleichgeblieben sind, wurden drei pensionierte Mitarbeiter nicht zur Gänze wiederbesetzt, sondern durch studentische Mitarbeiter_innen ersetzt, die vor allem die Abenddienste abdecken und sich um Paketpost, Ausfertigung und Bereitstellung der eingelangten Bestellungen für die Benutzer_innen kümmern. $\mathrm{Da}$ die Abenddienste in der Fernleihe, ganz im Gegensatz zur benachbarten Entlehnung, nicht besonders stark frequentiert wurden, lag eine verstärkte $\mathrm{Zu}$ sammenarbeit nahe, sodass die Entlehnung das Service Fernleihe in den Abendstunden ab 16 Uhr übernahm und Mitarbeiter_innen der Fernleihe in den Abenddienst der Entlehnung integriert wurden.

So gesehen hat sich in der Entlehnung und in der Fernleihe vieles getan, nur die Räumlichkeiten sind gleichgeblieben. Die Abteilungen haben gelernt, mit der Zeit zu gehen und auf veränderte Anforderungen zu reagieren. Dies wird im besonderen Maße mit der bevorstehenden Brandschutz-Sanierung der Hauptbibliothek ab 2021 weiterhin gefordert sein. 


\section{Die Lehrbuchsammlung - das versteckte Juwel}

Ein wenig versteckt liegt die Lehrbuchsammlung der Hauptbibliothek hinter der Feststiege $1 \mathrm{im}$ Hauptgebäude der Universität Wien. Das ist fast ein wenig schade, da sie mit ca. 40.000 Exemplaren im Vergleich zur Hauptbibliothek zwar klein ist, aber mit 62 Fachgebieten von der Anglistik über die Kristallographie bis zur Zoologie doch beinahe jeden (Lern-)Wunsch erfüllen kann.

Durch Neubauten von Fachbereichsbibliotheken in den vergangenen Jahren wurden die Bestände des Bereichs Informatik sowie die Fachgebiete Wirtschaftswissenschaften und Mathematik ausgegliedert. Ein Teil der Titel aus dem Bereich Kunstgeschichte wurde zwar an die entsprechende Fachbereichsbibliothek abgegeben, ein paar Jahren später aber wieder nachgekauft. Darüber hinaus wurde das Angebot der Lehrbuchsammlung um die Gebiete Religionswissenschaften und Bibliothekswissenschaft erweitert.

Die langjährige Statistikauswertung zeigte, dass ca. ein Drittel aller vorgemerkten Werke nach der Bereitstellung nicht abgeholt wurden. Dieser Wert war nicht zufriedenstellend, weshalb 2011 die Leihfrist der Lehrbuchsammlung von 56 Tagen auf 28 Tage verkürzt wurde. Wie die Statistik der nachfolgenden Jahre zeigt, blieb der angestrebte Effekt, eine Verkürzung der Wartezeit auf ein Buch und somit einen Rückgang der nicht abgeholten Bereitstellungen zu erreichen, jedoch aus. Die Werte sind annähernd gleichgeblieben.

Lange Zeit war die Entlehnung der Lehrbuchsammlungsbestände nur Studierenden vorbehalten. Die Lehrbuchsammlungen in den anderen Zweigstellen der Universitätsbibliothek konnten hingegen schon immer auch vom Personal der Universität Wien genutzt werden. Da in der Lehrbuchsammlung der Hauptbibliothek regelmäßig Ausnahmen für Mitarbeiter_innen der Universität gemacht wurden, wurde letztlich 2015 der Entschluss gefasst, die Ausnahme zur Regel zu machen und die reguläre Ausleihe für das Personal zu ermöglichen. Auf die Entlehnzahlen hat sich das deutlich ausgewirkt: Von ca. 200 Entlehnungen davor stiegen die Zahlen im ersten Jahr nach der Umstellung auf ca. 1.200, was einer Steigerung von $600 \%$ entspricht.

Aber nicht nur beim Bestand und in der Benutzung haben sich Dinge geändert, auch funktionell hat sich einiges getan. So wurden die schmalen Bücherablageplätze in den Galerien in vollwertige Leseplätze umgewandelt und im Raum 4 ein Gruppenarbeitsbereich eingerichtet, sodass in der Lehrbuchsammlung nun insgesamt 30 Arbeitsplätze zur Verfügung stehen. Im Zuge von Brandschutzmaßnahmen wurde die Garderobenpflicht zunächst für einige Monate aufgehoben. Da die damit gemachten Erfahrungen durchwegs positiv waren und der Bestand in der Lehrbuchsammlung aufgrund der Aktualität der Literatur und der Mehrfachexemplare nicht unersetzlich ist, wurde die Mit- 
nahme von Getränken und das Konsumieren von Speisen in den Räumlichkeiten erlaubt.

Von den Benutzer_innen, die den Weg in die Lehrbuchsammlung finden, werden die ausführliche Beratung durch die Mitarbeiter_innen, der ständige Bücherflohmarkt und der grandiose Blick auf den Rathauspark besonders geschätzt. 
Open-Access-Publikation im Sinne der CC-Lizenz BY-NC-ND 4.0

(c) 2019, Vandenhoeck \& Ruprecht GmbH \& Co. KG, Göttingen ISBN Print: 9783847110989 - ISBN E-Lib: 9783737010986 


\section{Formalerschließung von A bis Z. Universitätsbibliothek / Hauptbibliothek / Abteilung Formalerschließung}

Was haben Altbestandsbestellung und E-Books an der Universitätsbibliothek Wien gemeinsam? Sie werden in der Abteilung Formalerschließung bearbeitet, die organisatorisch zwar der Hauptbibliothek zugeordnet ist, mit ihren Aufgabengebieten aber einen darüberhinausgehenden Bereich von A wie Altbestandsbestellung, über $\mathrm{E}$ wie E-Books bis $\mathrm{Z}$ wie Zeitschriftenarchivierung abdeckt.

\section{A wie Altbestandsbestellung}

Das Service der Altbestandsbestellung wurde im Anschluss an das große RetroProjekt der Hauptbibliothek entwickelt, in dem der "Zettelkatalog" ohne Autopsie retrospektiv katalogisiert wurde. Als dieses Projekt Mitte 2008 abgeschlossen war, waren damit die Hauptbibliotheksbestände ab Erscheinungsjahr 1931 online erfasst. Die sehr umfangreichen Bestände vor 1931 konnten zu diesem Zeitpunkt aber weiterhin nur mittels Papierscheinen bestellt werden. Um den Leserinnen und Lesern die Bestellung dieser Bestände zu erleichtern, wurde in Abstimmung mit der Direktion im Jahr 2009 die Altbestandsbestellung in den Regelbetrieb übernommen. Worin bestand nun die Erleichterung? Die Leserinnen und Leser konnten ihre gewünschten Bücher via Online-Formular ordern. Das Team der Abteilung Formalerschließung übernahm mit diesem Service eine Schnittstellenfunktion zwischen der Leserin/dem Leser und den Abteilungen Büchermagazine, Entlehnung und Alte und wertvolle Bestände. Der seither zumeist reibungslose Ablauf der Altbestandsbestellung basiert auf einem guten Netzwerk im Haupthaus der Universitätsbibliothek Wien, dessen Linien sich in der Abteilung Formalerschließung kreuzen. Für die Erfüllung der täglichen Anforderungen der Altbestandsbestellung bedarf es manchmal sowohl kriminalistischen Gespürs, um etwa ausgefallene Leser_innenwünsche korrekt zu erfüllen oder zu interpretieren, als auch fundierten Wissens über die alten Kataloge und natürlich bester Beziehungen zu den Kolleginnen und Kollegen aus 
den anderen Abteilungen. Belohnt werden die Mühen manchmal mit skurrilen und amüsanten Fundstücken in den Büchern, wie vergessenen Liebesbriefen, Ansichtskarten oder antiquierten Bestellscheinen. Von all dem merkt die Leserin/der Leser nichts, denn das Service funktioniert und wird gut und immer besser angenommen. Durch die Altbestandsbestellung wird ergänzend zu den regulären Rückarbeiten von Altbeständen gleichzeitig eine von den Leser_innen bestimmte, kontinuierliche Retrokatalogisierung in kleinem Rahmen in der Abteilung Formalerschließung weitergeführt.

\section{E wie E-Books}

Als die ersten E-Books in den Bestand der Universitätsbibliothek aufgenommen wurden, stand zur Diskussion, ob dieses Medium in einem Bibliothekskatalog, der damals noch das Prädikat »ewig" trug, nachgewiesen werden soll. Denn bereits das erste E-Book-Paket war lediglich ein Testpaket, das nicht dauerhaft zugänglich bleiben würde. Die Entscheidung der Direktion fiel zugunsten des Nachweises im Online-Katalog aus, denn die Leserin/der Leser sucht ja in erster Linie ein Buch und nicht etwa ein E-Book oder ein Printbuch. Somit ergab sich, dass das Team der Abteilung Formalerschließung künftig auch für die Katalogisierung von E-Books zuständig sein würde. Die Herausforderung dabei war, diese neue Medienart in einer Umgebung zu katalogisieren, die auf Printmedien ausgelegt war. Dafür bekamen manche bestehenden MAB-Kategorien ${ }^{1}$ neue Funktionen, neue lokale Kategorien mussten »erfunden" werden. Auch im damaligen Regelwerk RAK-NBM ${ }^{2}$ musste sich diese neue Medienart irgendwie wiederfinden. Also versuchte man, neue Interpretationen aus alten Paragraphen herauszulesen. Wenn das nicht funktionierte, mussten österreichische Verbundregelungen verfasst werden. Darüber hinaus galt es, in enger Zusammenarbeit mit den Teams E-Resource Management und Bibliothekssysteme viele Fragen zu bedenken: Wie müssen Bestandsnachweise aussehen, damit die richtigen Buttons im Katalog entstehen? Wie geht man damit um, dass E-Books keiner Teilbibliothek zugehörig und in allen Zweigstellen und von zuhause aus nutzbar sind? Wie müssen die Metadaten beschaffen sein, damit eine größere Datenmenge bei Bedarf systemseitig auf einen Schlag korrigiert oder auch nicht zugänglich geschaltet werden kann? Eine Lösung dafür war zum Beispiel die Einführung von lokalen Paketsigeln als "Datenkennung». Daneben mussten auch neue Workflows implementiert werden, da E-Books natürlich einen an-

1 MAB: Maschinelles Austauschformat für Bibliotheken.

2 RAK-NBM: Regeln für die alphabetische Katalogisierung von Nichtbuchmaterialien. 
deren »Buchdurchlauf« - von der Erwerbung bis hin zur Benutzung - haben als gedruckte Bücher.

Neue Erwerbungsformen für E-Books bedeuteten für die Abteilung Formalerschließung ein enormes Anwachsen der Titelmengen und Datensätze, die zu bearbeiten und verwalten waren. Solange es um bewältigbare Titel- und Datenmengen ging, war manuelle Katalogisierung noch möglich. Als sich die Anzahl aber auf Hunderttausende steigerte, war an eine manuelle Datenerfassung nicht mehr zu denken. Also mussten Daten eingespielt werden, wofür man zusehends Metadaten heranzog, die von Verlagen angeboten wurden. Bei der Verwendung dieser Daten stellte das MAB-Format zu Aleph-Zeiten eine Hürde dar: Die Daten mussten konvertiert werden. Die Begutachtung und Bewertung der Metadaten aus diversen Quellen und die Überprüfung der Konvertierungsergebnisse fiel dem Team der Abteilung Formalerschließung zu, das damit bereits Jahre vor der Einführung von Alma erste Bekanntschaft mit dem MARCFormat ${ }^{3}$ machen durfte. Für die Bearbeiterinnen und Bearbeiter in der Abteilung Formalerschließung vollzog sich in den letzten zehn Jahren ein spannender und unerwarteter Wandel: Die Aufgaben erweiterten sich von der Katalogisierung einzelner E-Books um die intellektuelle Analyse der Metadaten tausender EBooks anhand repräsentativer Datensatz-Stichproben. Mit der Implementierung von Alma kamen unter anderem auch die Erstellung von Normierungsregeln und Importprofilen und die selbstständige Einspielung von Daten hinzu.

\section{Z wie Zeitschriftenarchivierung}

Durch einen deutlichen Zuwachs an besonders nachhaltig verfügbaren OnlineJournals im letzten Jahrzehnt eröffneten sich Möglichkeiten, die Bestände von Printzeitschriften zu schonen, die Aushebung und Entlehnung zu entlasten und gleichzeitig Magazinstellplatz zu gewinnen. Im Jahr 2014 stimmte die Direktion $\mathrm{zu}$, dass die Koordinationsstelle für Zeitschriftenarchivierung und -aussonderung eingerichtet und in der Abteilung Formalerschließung angesiedelt wurde. Ihre Aufgabe ist es unter anderem, bibliotheksweit, also unter Einbeziehung aller Zweigstellen, »beste« Bestände von Printzeitschriften zu bilden, die nachhaltig auch online verfügbar sind. Dabei müssen die Daten der Printmedien so aufbereitet werden, dass die Leserin/der Leser im Nachweissystem gezielt zur Benutzung der E-Journals hingeführt wird. Die betroffenen Printbestände können dadurch aus der Benutzung genommen und archiviert oder im Falle von Dubletten auch ausgesondert werden. Im Jahr 2018 konnte auch damit begonnen werden, Zeitschriftenbestände der Universitätsbibliothek, die bereits den Pro-

3 MARC: Machine-readable cataloging. 
zess der Zeitschriftenarchivierung durchlaufen hatten, in das neue (Übergangs-)Depot in Simmering umzusiedeln, um gezielt und in größerem Rahmen Magazinstellplatz zu gewinnen. Einige Mitarbeiterinnen und Mitarbeiter der Abteilung Formalerschließung erledigen vor Ort die Nacharbeiten dieser Umsiedlung so regelmäßig, dass man den Eindruck gewinnen könnte, es finden "kleine Betriebsausflüge « nach Simmering statt. In Zusammenarbeit mit dem Team E-Resource Management stehen weitere Bemühungen an, die Logistik beim Umzug umfangreicher Bestände zu unterstützen.

Man könnte das Alphabet durchaus mit weiteren Aufgaben aus dem Alltag der Formalerschließung füllen, das würde allerdings den Rahmen sprengen. Beleuchtet wurden hier bewusst nur die drei neuesten Schwerpunkte, die dank der Aufgeschlossenheit und Unterstützung von Maria Seissl eingeführt werden konnten. 


\section{Wohin mit all den Fragen? Team Infodienst und Führungen}

Wird eine Bibliothek tagtäglich von hunderten Menschen zu Studienzwecken besucht und werden tausende Personen jährlich durch Bibliotheksräumlichkeiten geführt, dann bestimmt das zum Großteil die Wahrnehmung derselben mit. Insofern ist es eine spannende Aufgabe, solche Prozesse über Jahre zu begleiten.

Die letzten 15 Jahre brachten eine Vielzahl von Veränderungen für das Team Infodienst und Führungen mit sich. Durch organisatorische und personelle Maßnahmen wurde das Team homogener, bauliche Veränderungen hatten ein moderneres und funktionelles Erscheinungsbild zum Ziel, Führungen können Benutzungsmodalitäten klären.

Das Team Infodienst und Führungen trägt die Verantwortung für drei Bereiche:

1. Bibliothekarische Information für die Benutzer_innen vor Ort zu gewährleisten. Der Informationsschalter (»Infopult») vor dem Großen Lesesaal wird dementsprechend mit Auskunftspersonal besetzt.

2. Angebote für Führungen und Schulungen für Gruppen zu entwickeln, zu organisieren und durchzuführen.

3. Literatur der Universitätsbibliothek für blinde und sehbeeinträchtigte Personen aufzubereiten und zur Verfügung zu stellen. Hierfür steht ein eigenes Literaturservice zur Verfügung.

Blicken wir zurück auf den Bereich des Infodienstes. Dienstpläne, die vor einem Jahrzehnt erstellt wurden, sprechen Bände: Vor rund zehn Jahren bestand der Infodienst noch vorwiegend aus akademischem Personal. Auskunft zu geben bedeutete, Literaturrecherchen durchzuführen. Um die gewünschte Literatur zu finden, wurden bibliografische Nachschlagewerke oder Zettelkataloge konsultiert. Manche Unzulänglichkeiten des Online-Katalogs konnten mittels bibliotheksspezifischen Fachwissens kompensiert werden. Komplexe Suchmaschinen steckten noch in den Kinderschuhen, stattdessen führten gezielte Suchen in Online-Katalogen zur geeigneten Lektüre. 
Vor fünfzehn Jahren wurden am Infopult noch Bestellscheine ausgefüllt und abgegeben. Sukzessive wurde die Onlinebestellung für diverse Bereiche eingeführt. Nach vielen Jahren, am 15. September 2010, wurden die letzten sich noch im Verkehr befindlichen Bestellscheine abgeschafft, die Onlinebestellung für den Lesesaal ging in Betrieb.

Das Erstellen von Dienstplänen für den Informationsdienst ist eine logistische Herausforderung, wird doch beinahe jeder Schalterdienst mit unterschiedlichen Personen besetzt, und zwar mit zwei Mitarbeiter_innen pro Diensteinheit. Der Auskunftsdienst wurde bis zum Jahr 2009 überwiegend von Fachreferent_innen bestritten. Eine Dienstplanänderung trat 2010 in Kraft: Akademisches Personal wurde vorwiegend nur noch in der Zeit von 12 bis $18 \mathrm{Uhr}$ eingesetzt, auch eine Folge der Umstrukturierung anderer Bereiche, wo Akademiker_innen nun vermehrt gebraucht und eingesetzt wurden. 2018 verbleiben nur noch drei Fachreferent_innen im Infodienst, der überwiegende Anteil wird von Mitarbeiter_innen des Teams bestritten.

Grund dafür ist sicherlich auch ein Wandel hinsichtlich der Auskunftstätigkeit. Studienrelevante Kompetenz ist von Vorteil, verstärkt ist jedoch Fachwissen in Bezug auf digitale Medien gefragt. Gleichzeitig sind formale Aufgabengebiete wie Garderobeschlüsselverwaltung und bestimmte Entlehntätigkeiten hinzugekommen. Auskunft wird weit über den bibliothekarischen Bereich hinausgehend $\mathrm{zu}$ allem, was "studienrelevant" ist, gegeben. Aber auch Fragen ohne Universitätsbezug kommen zuweilen vor. So wurde bereits die Frage nach der nächsten Tankstelle von findigen Infomitarbeiter_innen beantwortet. Flexibles Denken und Einfühlungsvermögen sind mitunter die wichtigsten Eigenschaften von qualifiziertem Auskunftspersonal!

Ergänzend muss der Umgang mit schwierigen Personen professionell gemeistert werden: Kleinere Vorfälle mit emotional belasteten Personen sind bei einer Tätigkeit im öffentlichen Raum immer an der Tagesordnung. Jede_r Infodienstmitarbeiter_in kennt sie: psychotische Personen, Stalker, Obdachlose. Seit 2016 ergänzen Securitymitarbeiter das Team und entlasten das Bibliothekspersonal in Situationen, mit denen sich die Mitarbeiter_innen regelmäßig konfrontiert sehen und die mit dem Auskunftsdienst an sich nichts zu tun haben. Eine datenschutzkonforme Installation von Überwachungskameras rundet das überarbeitete Sicherheitskonzept ab. Aber auch ein originärer Personenkreis an regelmäßigen Besucher_innen gehört wohl zu jeder Bibliothek. In der Hauptbibliothek zählen seit über einem Jahrzehnt der tägliche Wetterkartenleser, der ewige Student sowie die professionelle Querulantin dazu.

Den Wandel von Bibliotheken veranschaulichen auch bauliche Maßnahmen. 2010 wurden in zwei Räumen des Katalogsaals sechsundvierzig neue Sitzplätze geschaffen. Im Zuge des Umbaus kam es zur Bestandsaufnahme der dort auf- 
gestellten Werke und zu deren Retrokatalogisierung. Ein neues Aufstellungskonzept schuf Platz für dringend benötigte Lernplätze.

Im November 2012 wurde der bis dahin zum Publikumsbereich gerechnete, aber doch sehr abgelegene vierte Katalogsaal dem internen Magazinsbereich zugeschlagen. 2014 wurden im Katalogsaal achtzehn Recherche-Computer gegen PCs des Zentralen Informatikdienstes getauscht, um vielfältigere Arbeitsmöglichkeiten für die Benutzer_innen zu schaffen. Über die Sommermonate 2017 erfolgte die Renovierung des Glasdaches des Großen Lesesaals. 2018 wurde der Kleine Lesesaal in Büroräumlichkeiten umgebaut, als Ersatz entstand die Studienzone im Atrium Hof 7.

Das Team Infodienst und Führungen bietet für Gruppen Bibliotheksführungen sowie Rechercheschulungen für Schulklassen an. Diese beinhalten Informationen $\mathrm{zu}$ Benutzungsmodalitäten und Recherchetools sowie einen Rundgang durch die Räumlichkeiten vor Ort. Zielgruppen sind vor allem Studierende und Schulklassen. Gerne gebucht werden diese auch von Bibliothekar_innen aus dem Ausland.

Die Statistik der letzten 15 Jahre zeigt, dass Führungen und Schulungen seit 2004 gestiegen sind, insbesondere 2012.

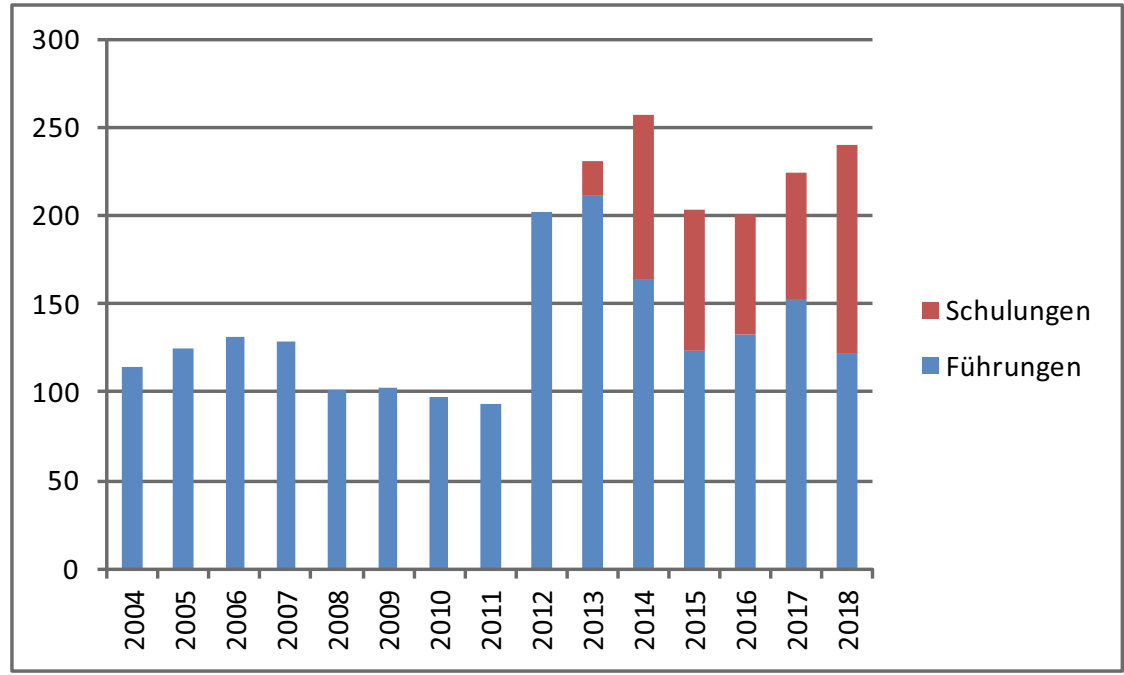

Entwicklung der Führungen und Rechercheschulungen 2004-2018

In diesem Jahr kam die im Kontext der Matura ab dem Schuljahr 2014/2015 an den Allgemeinbildenden höheren Schulen eingeführte »Vorwissenschaftliche Arbeit« (VWA) bzw. die Diplomarbeit (DA) an den Berufsbildenden höheren Schulen erstmals zum Tragen. Seit November 2013 bietet das Team Infodienst und Führungen zusätzlich Rechercheschulungen für Schulklassen an. 
Das dritte Aufgabengebiet soll nicht unerwähnt bleiben: das Literaturservice für blinde und sehbeeinträchtigte Menschen. Mitarbeiter_innen des Literaturservices bereiten in der Universitätsbibliothek vorhandene Medien auf eine adäquate Weise für blinde und sehbeeinträchtigte Personen auf. Viele Jahre über von zwei Mitarbeiter_innen betreut, wurde die Nachfrage nach Literatur vor ca. fünf Jahren so groß, dass es zu Umstrukturierungen kam: Einerseits wurden zusätzliche Mitarbeiter_innen für die Bearbeitung der Texte angestellt, andererseits wurden die Qualitätsstandards der Textaufbereitung höher gesetzt. Inklusion bedeutet für Bibliotheken, für qualitative Literaturversorgung blinder Personen Sorge zu tragen.

Im täglichen Kontakt mit den Benutzer_innen wird die Notwendigkeit von Veränderungen augenscheinlich. Nur durch eine beherzte Bibliotheksleitung sind Innovationen möglich. Die letzten fünfzehn Jahre haben für das Team Infodienst und Führungen eine Vielfalt von Erneuerungen möglich gemacht. 


\section{Fachbereichsbibliotheken}


Open-Access-Publikation im Sinne der CC-Lizenz BY-NC-ND 4.0

(c) 2019, Vandenhoeck \& Ruprecht GmbH \& Co. KG, Göttingen ISBN Print: 9783847110989 - ISBN E-Lib: 9783737010986 


\section{maktaba yangu - مكتبتي - obá akwụkwo m - benim kütüphanem - كتابخانه مني Die Fachbereichsbibliothek Afrikawissenschaften und Orientalistik}

"Meine Bibliothek" in einigen Sprachen, die an der Fachbereichsbibliothek Afrikawissenschaften und Orientalistik vertreten sind. Meine Bibliothek im Sinne von einem Ort zum Wohlfühlen, quasi ein zweites Wohnzimmer für unterschiedliche Menschen, die hier lesen, lernen, kommunizieren und/oder Bücher entlehnen wollen.

\section{Wie alles begann ...}

Die Fachbereichsbibliothek Afrikawissenschaften und Orientalistik geht in der heutigen Gestalt auf den Oktober 2005 zurück, als die bis dahin existierende Fachbereichsbibliothek Afrikanistik und die Institutsbibliothek Orientalistik zusammengelegt wurden.

Zwei Monate genügten, um zwei Hörsäle und Büros des Instituts für Orientalistik zu Bibliotheksräumlichkeiten umzubauen. Die so neu geschaffenen Bereiche wurden mit der bereits bestehenden Fachbereichsbibliothek Afrikanistik verbunden. Entstanden waren vier in Schlauchform angeordnete Bibliotheksräume, die über das Erdgeschoß des Instituts für Orientalistik zugänglich waren. Die bis dahin auf sieben Büros aufgeteilte - und von Mitarbeiter_innen des Instituts betreute - Bibliothek für Orientalistik wurde in die Dienstleistungseinrichtung Bibliotheks- und Archivwesen eingegliedert.

Die Fachbereichsbibliothek umfasste Ende 2005 einen Bestand von 67.000 Bänden und 700 Zeitschriften, etwa ein Drittel davon laufend gehalten. Erich Sommerauer leitete - wie seit 1978 die vormalige Instituts- bzw. Fachbereichsbibliothek Afrikanistik - die neue Bibliothek, David Parsian fungierte als sein Stellvertreter und die Autorin dieses Beitrags war geringfügig beschäftigt.

Die Bibliothekszusammenlegung brachte für alle Beteiligten große Veränderungen mit sich. Sieben verschiedene Systematiken (Arabica, Turcica, Semitica, Assyrica, Zeitschriften Orientalistik, Zeitschriften Afrikanistik, Bestände der Afrikanistik / später umbenannt in Afrikawissenschaften) befanden sich nun 
unter einem Dach. Der Eingang wurde von Hof 5 in den Hof 4 verlegt und in der Bibliothek gab es nun fünf Glastüren zwischen den einzelnen Räumen, die täglich unzählige Male (auch mit Bücherwagen) durchschritten werden mussten. Eine einheitliche Entlehnung über das Bibliothekssystem Aleph löste das bis dahin übliche Ausfüllen eines Entlehnscheins und die Stellvertreter im Regal ab. Der große Vorteil der neuen Bibliothek war unbestritten: ausreichend Leseplätze, verlängerte Öffnungszeiten und ein computergestütztes Entlehnsystem.

Für die Mitarbeiter_innen bedeutete die Zusammenlegung ein Sich-Einlassen auf deutlich mehr Benutzer_innen, neue Themenbereiche und Fragen, die fernab des eigenen Studiums lagen. Aber an Herausforderungen wächst man! Die Buchbestände waren zu diesem Zeitpunkt nur teilweise im Online-Katalog erfasst, Kurztitelaufnahmen und Rückarbeit standen auf der Tagesordnung. Zettelkataloge im ersten und vierten Raum schufen Abhilfe für die Recherche nach all jenen Büchern, die noch nicht in Aleph erfasst waren.

Zum Abschluss der Bibliothekszusammenlegung noch eine historische Reminiszenz: nach 112 Jahren waren die beiden Institute in Form der Fachbereichsbibliothek wieder vereint. Denn zwischen 1893 und 1922/23 fanden afrikanistische Vorlesungen am orientalischen Institut unter der Studienrichtung »allgemeine und vergleichende Sprachforschung und orientalische Philologie» statt. 1923 folgte die Gründung des Instituts für Ägyptologie und Afrikanistik, von dem sich 1978 ein eigenes Institut für Afrikanistik samt dazugehöriger Bibliothek abspaltete. Tempora mutantur?

\section{Personalia}

Die Zeiten ändern sich, aber manche Mitarbeiter_innen bleiben der Bibliothek erhalten. Nach der Pensionierung des bisherigen Leiters, Erich Sommerauer, im Herbst 2008 folgte eine zweijährige Übergangszeit mit provisorischer Leitung durch den damaligen stellvertretenden Leiter der Dienstleistungseinrichtung Bibliotheks- und Archivwesen Andreas Brandtner. In dieser Zeit wurde die Arbeitszeit der bis dahin geringfügig beschäftigten Autorin dieses Beitrags auf 30 Wochenstunden erhöht und die studierte Afrikanistin begann mit einer bibliothekarischen Ausbildung im Rahmen des Universitätslehrgangs Library and Information Studies.

Im Oktober 2010 übernahm sie die Leitung der Fachbereichsbibliothek, David Parsian wechselte ins Universitätsarchiv und der promovierte Orientalist Usama Maher verließ die Hauptbibliothek, um sich an der Fachbereichsbibliothek einzubringen. 


\section{Und heute ...}

... befindet sich die Fachbereichsbibliothek räumlich immer noch im mittlerweile 20 Jahre alten Campus der Universität Wien. Das Service und die Rahmenbedingungen haben sich in den letzten Jahren jedoch deutlich verbessert.

Ein Projekt im Rahmen des Universitätslehrgangs Library and Information Studies hat sich 2008/09 im Hinblick auf Qualitätssicherung mit den Bedürfnissen der Benutzer_innen dieser Fachbereichsbibliothek auseinandergesetzt. Eine Fotobefragung, bei der zwölf Fragen nach Wünschen und Bedürfnissen der Leser_innen mit einem Foto beantwortet werden sollten, ermöglichte einen Blick in die Bibliothek durch die Linse der Benutzer_innen. Der anschließende »Library Design Workshop« plante den Entwurf einer »idealen« Bibliothek auf dem Reißbrett.

Eindeutiger Wunsch der Benutzer_innen war ein ruhiger Lernort, der mit Pflanzen, Kaffeeautomat und Sofa gemütlicher gestaltet werden sollte. Längere Öffnungszeiten und verbesserte Entlehnmöglichkeiten waren ebenso wichtige Anliegen wie ein übersichtliches Leitsystem, um sich mit den zahlreichen Systematiken und der ungünstigen Anordnung der Räumlichkeiten besser zurechtzufinden. Gesagt, getan!

Heute hat die Fachbereichsbibliothek einen Großteil der Anliegen umgesetzt und präsentiert sich als gemütlicher Lernort in fast familiärer Atmosphäre. Hier kennen wir Bibliotheksmitarbeiterinnen und -mitarbeiter einen Großteil der Benutzer_innen, die uns oft jahrelang besuchen. Das markante rote Sofa im Raum 4 lädt zum Entspannen zwischendurch ein und ein Kaffee aus dem Automaten im Garderobenraum hilft über ein mögliches Nachmittagstief hinweg. Eine Kinderecke und die Möglichkeit, Spielzeugrucksäcke auszuborgen, schaffen ein familienfreundliches Klima.

Die Öffnungszeiten wurden deutlich ausgeweitet und ermöglichen auch Berufstätigen einen verbesserten Zugang. Das Leitsystem wurde komplett überarbeitet und jeder Fachbereich hat nun eine übersichtliche und farblich markante Beschriftung.

Die geblockte Aufstellung des afrikawissenschaftlichen Bestands wurde neu organisiert, die alphabetische Ausrichtung der Bücher dem Eingang in Hof 4 angepasst. Mehr Platz, besserer Lichteinfall und mehr Einzelarbeitsplätze öffneten den Raum und verschafften ihm einen deutlichen Qualitätsgewinn.

Mit der Eröffnung eines Gruppenarbeitsraums im Jahr 2013 wurde die Bibliothek um einen Raum erweitert und damit ein lange gehegter Wunsch erfüllt. In diesem ehemaligen Büro ist nun auch abseits des Garderobenraums Platz für Gemeinschaftsprojekte und lautstarke Diskussionen.

Abschließend könnte man sagen: wir sind zusammengewachsen. Die Rahmenbedingungen der letzten gut zehn Jahre haben das möglich gemacht. In der 
Fachbereichsbibliothek Afrikawissenschaften und Orientalistik treffen täglich viele unterschiedliche Menschen aufeinander: Das Bibliothekspersonal, das wissenschaftliche Personal und Studierende verschiedener Fachbereiche ebenso wie Walk-In-User, die neugierig auf die Bibliothek oder auf der Suche nach einem Tandempartner (zwei Muttersprachler lernen voneinander) sind. Wir sind eine Bibliothek für all diese Benutzer_innen. Egal, ob jemand klassisch Bücher ausborgt bzw. zurückbringt, Freund_innen oder Studienkolleg_innen trifft, sich auf der Couch eine halbe Stunde hinlegt, betet, zum Lernen oder Lesen kommt. 


\section{Nicht von Gestern. Die Fachbereichsbibliothek Alte Geschichte}

Das Profil der Bibliotheksbenutzer_innen der Fachbereichsbibliothek Alte Geschichte hat sich in den letzten Jahrzehnten stark gewandelt. Waren es in den 1980er Jahren ausschließlich Studierende der eigenen Fachrichtung, änderte sich das langsam aber stetig ab dem Ende der 1990er Jahren mit der Ablöse des Zettelkataloges und der immer größer werdenden Bedeutung der EDV und der Einführung der Entlehnung. Die Motivation, den ganzen Tag am Institut zu verbringen, verringerte sich - und damit auch die zwischenmenschliche Kommunikation. Die Arbeit mit und am Computer zu Hause wurde immer wichtiger, die Recherche- und Entlehnmöglichkeiten wurden erweitert und modernisiert. Durch das Ansteigen der Entlehnungen sank die Anzahl der entwendeten oder »irrtümlich« ausgeliehenen Bücher deutlich; sukzessive kam mehr Transparenz in die Bibliothek.

Seit den 2010er Jahren besuchen immer mehr fachfremde Studierende die Räumlichkeiten der Bibliothek, sei es um zu lernen, sei es um ein ruhiges Plätzchen vor der nächsten Vorlesung zu finden. Auch zwei Schachspieler schätzen im Winter ein abgelegenes Plätzchen. Literatur findet man mittlerweile sehr gut in dem großen Angebot der elektronischen Medien. Viele Lehrveranstaltungsleiter_innen scannen Hausübungen oder Prüfungstexte ein und stellen sie auf die E-Learningplattform Moodle. Und dennoch verliert das Buch in dem Bereich der Altertumswissenschaft seinen Stellenwert nicht.

Die Bibliotheksnutzer_innen schätzen Bücher neben digitalen Medien und Zeitschriften weiterhin sehr: Man kann es anfassen, darin blättern, immer wieder ausborgen. Es kommt bei den Printexemplaren aber auch immer wieder vor, dass sie bewusst ins falsche Regal gestellt werden, damit man selbst exklusiv Zugriff hat. Und schließlich sind Bücher auch immer wieder ein geeignetes Objekt, um damit zur Bibliothekarin zu gehen und festzustellen, was für einen unmöglichen Autor sie da gekauft habe. 
Alle, die einmal in der Fachbereichsbibliothek Alte Geschichte gearbeitet, gelesen, studiert haben, erinnern sich sehr gerne an diese Zeit zurück. ${ }^{1}$ Die thematische Aufstellung der Fachbereichsbibliothek ist schnell zu verstehen, die Anzahl der Arbeitsplätze zwar beschränkt, aber mit gutem Licht, der Flirtfaktor wurde in einem anlässlich des Jubiläumsjahres 2015 produzierten Bibliotheksquartetts bewertet: Die Fachbereichsbibliothek Alte Geschichte erhielt die Bestnote von fünf Herzen.

Möglichkeiten zum Kopieren und seit Kurzem auch zum Scannen sind vorhanden. Durch die Gemeinsamkeit der Nutzung der Räume mit dem Institut geht es nicht immer leise zu, dennoch schafft diese Symbiose von Institut und Fachbereichsbibliothek eine wohlige, wohnzimmerartige Atmosphäre, in der die Benutzer_innen gut lernen und wissenschaftlich arbeiten können. Dies unterstreicht das 2018 erfolgte Ranking der Topuniversitäten, ${ }^{2}$ in dem es die Universität Wien als beste heimische Universität in 16 Fächern unter die Top 100 und in fünf unter die Top 50 schaffte. Die beste Platzierung im Bereich der Institute fiel auf Platz 26 für das Fach Alte Geschichte und Altertumskunde in der Abteilung "Classics and Ancient History«. Selbstredend hat auch die Fachbereichsbibliothek Anteil an diesem Erfolg.

Ein wichtiger und schöner Punkt im Arbeitsfeld als Bibliothekarin der Fachbereichsbibliothek Alte Geschichte besteht in der Begleitung junger Menschen vom ersten Semester bis zu ihren akademischen Abschlüssen, Projekten oder Anstellungen, und in dieser Zeit nicht selten durch Höhen und Tiefen. Zu den aktiven Benutzer_innen gehören vermehrt auch Schüler_innen, die eine Vorwissenschaftliche Arbeit verfassen. Gelegentlich kommen freilich ihre Mütter und Väter, die - dann meist telefonisch und in letzter Minute - für die Vorwissenschaftliche Arbeit ihrer Kinder Literatur suchen, kurz vor der Schließzeit hektisch Bücher entlehnen und diese auch wieder selbst zurückbringen.

Mit dem Profil der Bibliotheksbenutzer_innen haben sich auch die Aufgaben und Anforderungen an die Bibliothekar_innen in den letzten Jahren gewandelt. Diese Veränderungen machen es zunehmend schwierig, die an der Fachbereichsbibliothek lange Zeit praktizierte Unterstützung durch Institutspersonal im Infodienst aufrechtzuerhalten. Dennoch ist die Bibliothek an dem Fortbestand der Symbiose mit dem Institut interessiert, da viele Benutzer_innen eine offene Atmosphäre sehr schätzen: das Miteinander reden, Vorträge halten, Fachprobleme lösen, Diskutieren (natürlich auch über Neuerwerbungen, Öff-

1 Cornelius Volk: Krisenphänomene im nördlichen Kleinasien des 3. Jahrhunderts nach Christus. Wien: Masterarbeit, 2018: »In den zwei Jahren, die ich bisher hier bin, habe ich Bibliothek und Institut schätzen gelernt und fühle mich dort bis heute ungemein wohl«.

2 https://www.topuniversities.com/university-rankings/university-subject-rankings/2018/clas sics-ancient-history [Letzter Zugriff: 11.09.2019]. 
nungszeiten, Ersatzdienste). Nicht zuletzt soll auch das ein wertvoller Bestandteil der universitären Ausbildung sein, denn universitas bedeutet Gemeinschaft und in diesem Sinne wird die Fachbereichsbibliothek Alte Geschichte sich weiterhin darum bemühen, eine Bibliothek der Begegnungen zu bleiben. 
Open-Access-Publikation im Sinne der CC-Lizenz BY-NC-ND 4.0

(c) 2019, Vandenhoeck \& Ruprecht GmbH \& Co. KG, Göttingen ISBN Print: 9783847110989 - ISBN E-Lib: 9783737010986 


\section{Same same or different? Die Fachbereichsbibliothek Anglistik und Amerikanistik 2004-2019: Vor der Bibliothek muss man sich nicht fürchten}

Ist es wirklich schon 15 Jahre her, dass die Universitätsbibliothek Wien mit Maria Seissl eine neue Leiterin erhielt? 2004 war auch das Jahr, in dem die Autorin den Universitätslehrgang Library and Information Studies begann, dessen Abschluss bald die Grundlage für ihre Bewerbung und Tätigkeit als Leiterin der Fachbereichsbibliothek Anglistik und Amerikanistik bildete.

Seit dieser Zeit wurde an der Universitätsbibliothek insgesamt und an der Fachbereichsbibliothek im Besonderen vieles bewegt, verändert, auch laut Rückmeldungen von Benutzer_innen verbessert.

Leitgedanken für die Neugestaltung der Fachbereichsbibliothek waren die Vorgaben und Ideen der Direktion dafür, wie die Services einer zeitgemäßen benutzerorientierten Fachbereichsbibliothek aussehen sollten. Das Engagement und die Bereitschaft der Mitarbeiterinnen, sich auf Neues einzulassen und selbständig das Potential für Verbesserungen zu erkennen, hat das übrige dazu beigetragen, dass die Fachbereichsbibliothek in vielerlei Hinsicht eine andere Bibliothek ist, als die, die die Autorin selbst als Studierende genutzt hat. Im Fokus standen dabei die Nutzer_innen der Bibliothek. Anders als während der Zeit, die die Autorin als Studierende miterlebte, sollte sich niemand mehr davor fürchten, die Bibliothek und ihre Bestände zu nutzen.

\section{Was hat sich verändert? Eine Bilanz}

Mit der Pensionierung des Leiters der Fachbereichsbibliothek und einer weiteren Mitarbeiterin sowie der Anstellung der Autorin als neuer Leiterin sowie der Aufnahme einer neuen Mitarbeiterin ging die Personalbesetzung der Fachbereichsbibliothek ganz in die Verantwortung der Universitätsbibliothek über. In den Folgejahren kamen zum Kernteam auf Grund der Erweiterung der Öffnungszeiten der Fachbereichsbibliothek eine Reihe geringfügig beschäftigter Mitarbeiterinnen hinzu. Aufgaben der Fachbereichsbibliothek werden mit Hilfe der Unterstützung der Fachbereichsbibliothek Judaistik (Schalter) und 
des Teams Integrierte Medienbearbeitung Fachbereichsbibliotheken (Erwerbung und formale Erschließung von Medien) sowie anderer zentraler Services der Universitätsbibliothek geleistet.

Bei allen Veränderungen, die an der Fachbereichsbibliothek selbst vorgenommen wurden, ging es in erster Linie darum, die Services der Bibliothek für Benutzer_innen und im Fachbereich angesiedelte Personen zu verbessern. Andere Veränderungen ergaben sich durch gesamtbibliothekarische und über die Universitätsbibliothek Wien hinausgehende Entwicklungen von Services und technischer Infrastruktur:

Auf- und Ausbau der Fachbereichsbibliothek als Teaching Library: Auf Grund von Kooperationen mit Lehrenden der Anglistik und Amerikanistik konnte die Bibliotheksleiterin ein Workshopangebot für verschiedene Bereiche und Stufen der Studienprogramme entwickeln, das aus direkt in Lehrveranstaltungen integrierten oder aus sie begleitenden Veranstaltungen besteht. Das Angebot wurde und wird unter Heranziehen von Best-Practice-Beispielen und internationalen Standards und Konzepten der Teaching Library weiterentwickelt. Auch bei Willkommensveranstaltungen für neue Studierende wird die Bibliothek als Informationsressource präsentiert. Weiters wurde von der Bibliotheksleiterin und Lehrenden aus verschiedenen Fachbereichen der Anglistik und Amerikanistik eine Massenlehrveranstaltung mit zwei ECTS-Punkten zum Thema Informations- und Recherchekompetenz für das Bachelorstudium Unterrichtsfach Englisch entwickelt. Seit 2015 finden ein bis zwei dieser Lehrveranstaltung mit insgesamt ca. 50-260 Teilnehmer_innen pro Semester statt. Zu den Lernzielen dieser Lehrveranstaltung gehört es, die Fachbereichsbibliothek und andere Bibliotheken sowie allgemeine und fachbezogene Sucheinstiege und Informationsressourcen aus dem Angebot der Universitätsbibliothek kennen und nutzen zu können. Alle Teilnehmer_innen besuchen die Bibliothek als Teil ihrer zu erbringenden Leistungen.

Öffnungszeiten der Fachbereichsbibliothek: Durch eine Erweiterung des Teams und die Zusammenarbeit mit der Fachbereichsbibliothek Judaistik konnte eine Erweiterung der Öffnungszeiten auf 50 Stunden pro Woche im Semester (Montag bis Freitag) erreicht werden.

Entlehnung: Die Fachbereichsbibliothek entwickelte sich im beschriebenen Zeitraum von einer mehr auf Bestandsbewahrung ausgerichteten Wochenendbibliothek zu einer 7-Tage-Entlehnbibliothek.

Bestandsnachweis: Alle nur im Kartenkatalog erfassten nutzbaren Bestände der Fachbereichsbibliothek wurden durch das Retrokatalogisierungsprojekt der Universitätsbibliothek in Aleph retrokatalogisiert und damit zunächst für Benutzer_innen im Bibliothekskatalog suchbar gemacht. Der Kartenkatalog hat daher an der Fachbereichsbibliothek als Suchinstrument ausgedient. Wie bei 
allen anderen Teilbibliotheken auch erfolgte der Umstieg vom Online-Katalog auf $\mathrm{u}$ :search, und der Umstieg auf das neue Bibliothekssystem Alma.

Webauftritt: Der Umstieg von einer eigenen Website auf eine Unterseite der Website der Universitätsbibliothek bedeutete, dass Services der Bibliotheken für alle Benutzer_innen leichter als solche erkennbar wurden.

Verbesserung und Anpassung des Leitsystems und der Orientierungshilfen: Von Anfang an wurde vom Bibliotheksteam versucht, die Auffindbarkeit von Beständen zu verbessern, z. B. indem farbkodierte Signaturenbeschriftungen am Regal eingeführt wurden. Im Zuge der Einrichtung eines Zugangs zur Fachbereichsbibliothek Judaistik über die Fachbereichsbibliothek Anglistik kam es zu einer Neuausschilderung und einem professionellen Leitsystem für alle Bereiche der Bibliothek.

Zusammenarbeit mit der Fachbereichsbibliothek Judaistik: Durch bauliche Maßnahmen wurde ein Durchgang zwischen den beiden Bibliotheken geschaffen. Ab $13 \mathrm{Uhr}$ ist die Fachbereichsbibliothek Judaistik über die Fachbereichsbibliothek Anglistik und Amerikanistik erreichbar, deren Schalter dann für beide Bibliotheken zuständig ist. Durch die Kooperation am Schalter wurde beiden Bibliotheken ein längeres Offenhalten ermöglicht.

Ausbau der Freihandaufstellung: Der Zugang zu unseren nutzbaren Büchern und Zeitschriften wurde verbessert. Früher getrennt aufgestellte Magazins- und Freihandbestände mit der gleichen Signatur wurden gemeinsam in den für Benutzer_innen zugänglichen Bereichen der Bibliothek aufgestellt, wobei ein früheres Magazin und Büroräume als Lesesäle umgestaltet wurden. Eine Magazinsaufstellung wird nur mehr aus archivarischen Gründen vorgenommen.

Schulbücher: Für die Aufstellung und Nutzung der aktuellen Schulbücher für das Unterrichtsfach Englisch wurde ein eigener Raum freigemacht. Durch die dankenswerte Unterstützung der Fachdidaktik für das Unterrichtsfach Englisch bei der Beschaffung aktueller Titel (Schenkung), ist es möglich, den Studierenden aktuelle Schulbücher und dazugehörige Medien vor Ort zugänglich zu machen.

Gesprächs- und Ruhearbeitsbereiche: Um unterschiedlichen Bedürfnissen von Bibliotheksnutzer_innen nachzukommen, wurden Räume der Bibliothek zu Gesprächs- oder Ruhezonen erklärt.

Archivierung des gebundenen Zeitschriftenbestandes: Dort, wo Printzeitschriften gesichert online vorhanden sind, wurden sie aus der Freihandaufstellung entfernt und im Magazin archiviert. Dadurch konnte für andere Bestände Platz gewonnen werden. 


\section{Über den Tellerrand hinaus}

Über das bibliothekarische Alltagsgeschäft hinaus sollen zwei Highlights erwähnt werden, die in den vergangenen 15 Jahren eine besondere Bedeutung bekommen haben:

Die Arbeit des Projekts und des Teams Provenienzforschung wurde von der Fachbereichsbibliothek dabei unterstützt, die aus den Beständen der Bibliothek der APA (All Peoples' Association) unrechtmäßig in den Bestand der Fachbereichsbibliothek übergegangenen Werken zusammenzutragen. Da es sich um sogenanntes »erbloses« Gut handelte, erfolgten Übergabe und Rückkauf in Kooperation mit dem Nationalfonds der Republik Österreich für Opfer des Nationalsozialismus. Der bezahlte Betrag kam damit, wie vertraglich für solche Fälle vereinbart, NS-Opfern zugute. 2018 wurden die rückgekauften Bücher im Freihandbereich unter der Signatur APA gesondert aufgestellt, um auf die besondere Provenienz hinzuweisen. Informationstafeln erklären den Fall, wodurch vor Ort auf den Umgang der Universitätsbibliothek mit dieser Problematik hingewiesen wird.

Seit 2005 findet in Kooperation mit dem Wiener Kulturverein Vienna Lit einmal im Monat ein Buchgruppentreffen statt, um über ein englischsprachiges zeitgenössisches Werk zu diskutieren. An diesen Events haben über die Jahre Personen aus dem Fachbereich, aber auch viele Menschen mit ganz verschiedenem nationalem und beruflichem Hintergrund teilgenommen. Dadurch ist die Bibliothek auch über die Fachgrenzen hinaus zu einem Ort des kulturellen Austauschs zur anglophonen Literatur geworden.

\section{Ausblick}

Wie auch in der Vergangenheit wird sich die Fachbereichsbibliothek den Herausforderungen, die da kommen mögen, stellen. Was für die Autorin schon feststeht: Weiterhin werden die Benutzer_innen der Bibliothek im Mittelpunkt stehen. 


\section{Eine Bibliothek mit Weitblick. Die Fachbereichsbibliothek Archäologien und Numismatik}

Die Fachbereichsbibliothek Archäologien und Numismatik umfasst vier Teilbibliotheken, die in der Franz-Klein-Gasse im 19. Bezirk angesiedelt sind - mit unmittelbarem Ausblick auf den wunderschönen Währingerpark und spektakulärem Weitblick auf die Stadt sowie den Leopoldsberg und den Kahlenberg.

Die Bibliotheken wurden in zwei Schritten organisatorisch zusammengelegt. Zunächst kam es 2008 zum Zusammenschluss der bereits seit 1988 vor Ort angesiedelten Bibliotheken der Fachbereiche Urgeschichte und Historische Archäologie (vormals Ur- und Frühgeschichte), Klassische Archäologie sowie Numismatik und Geldgeschichte unter der gemeinsamen Bezeichnung Fachbereichsbibliothek Archäologien und Numismatik. Im Frühjahr 2014, mit der Übersiedlung des Instituts für Ägyptologie in das Archäologiezentrum in der Franz-Klein-Gasse, wurde auch die Teilbibliothek Ägyptologie (bis zu diesem Zeitpunkt als Institutsbibliothek geführt) dem Verband der Fachbereichsbibliothek eingegliedert.

Damit sind nunmehr die Bibliotheken aller "grabenden Disziplinen ${ }^{1}$ unter einem Dach vereint und administrativ zusammengefasst, wiewohl sie nicht gemeinsam in zusammenhängenden Räumen untergebracht sind. Jeder der vier Teilbibliotheken stehen Räumlichkeiten innerhalb der jeweiligen Institute zur Verfügung, wie das auch schon vor der Zusammenlegung der Fall war.

Natürlich fördert diese weiterhin bestehende räumliche Verknüpfung nach wie vor das rege Interesse der Institute an der Gestaltung und dem Gebaren der Bibliotheken. Geplante Änderungen werden intensiv kommuniziert, teilweise zuvor mit Ansprechpartnern der Institute diskutiert und in Absprache umgesetzt. Die enge Zusammenarbeit von Instituten und Bibliotheken manifestiert sich auch darin, dass die Betreuung der Bibliotheksbenutzer_innen an zwei Standorten, nämlich Numismatik und Ägyptologie, nur mit Unterstützung durch das Institutspersonal möglich ist. In diesen beiden Bibliotheken wurden bzw. werden verschiedene bibliothekarische Tätigkeiten auch nach den Ein-

1 ๑ Gabriela Krämer, erste Leiterin der zusammengelegten Bibliotheken von 2008 bis 2013. 
gliederungen in die Fachbereichsbibliothek weiterhin von Mitarbeiter_innen der Institute durchgeführt. In den vergangenen Jahren hat das Bibliotheksteam aber zahlreiche der ehemals bei den Instituten angesiedelten Arbeitsschritte nach und nach übernommen. Die Arbeit der Fachbereichsbibliothek wird dadurch in vielen Bereichen immer sichtbarer, durch entsprechendes Engagement der Mitarbeiter_innen werden kontinuierlich Akzeptanz und Vertrauen in ihre Arbeit aufgebaut.

Die gute Zusammenarbeit zwischen Bibliothek und Instituten macht sich auch in ganz anderen Bereichen bemerkbar: Die üppige Pflanzenpracht an unserem wunderschönen, riesengroßen Panoramafenster am Standort Urgeschichte im 4. Stock wäre wohl bei Weitem nicht so prächtig, würden wir nicht von einem professoralen Institutsangehörigen, der sich als ausgewiesener Pflanzenkenner und -liebhaber entpuppt hat, regelmäßig mit Regenwasser aus seinem Garten versorgt, mit dem unsere Pflanzen - ausschließlich! - gegossen werden. Einmal wöchentlich ist deshalb eine Fachbereichsbibliotheksmitarbeiterin damit betraut, aus dem »Säurekammerl« des Instituts, in dem ansonsten die Chemikalien für die Labors aufbewahrt werden, das in Kanistern abgefüllte Regenwasser zu holen, das dort für unsere Pflanzen bereitgestellt wird. $\mathrm{Zu}$ Verwechslungen der Kanister kam es glücklicherweise noch nie ...

Die Fachbereichsbibliothek betreut in ihrer Vielfalt und in ihrem Umfang einen im Vergleich mit anderen fachlich gleichartigen Institutionen eindrucksvollen Bestand, der zurzeit ca. 133.000 Bände umfasst. Dieser Bestand ist im Laufe der Jahrzehnte nicht nur durch käuflichen Erwerb angewachsen, sondern ebenso durch die sehr intensiven Tauschbeziehungen zu zahlreichen archäologischen Einrichtungen europaweit. Die Bibliothek kommt so auch an Literatur, die über herkömmlichen Weg oft nicht (mehr) beschaffbar wäre.

Zusätzlich zum gut ausgestatteten physischen Buchbestand profitieren die Nutzer_innen der Fachbereichsbibliothek auch vom umfangreichen E-Ressourcen-Angebot der Universitätsbibliothek Wien. Zwar wurde bei den traditionell den »Buchwissenschaften « zuzurechnenden archäologischen Fächern die vermehrte Präsenz von E-Medien zunächst skeptisch gesehen; die Etablierung der Digital Humanities im Arbeitsbereich der archäologischen Wissenschaften bringt es aber mit sich, dass das elektronische Angebot immer mehr als zusätzliche Informationsquelle genutzt und geschätzt wird. Es ist als ein wesentlicher Auftrag der Fachbereichsbibliothek zu sehen, diese neuen Entwicklungen der sich verändernden Wissensbereitstellung den Nutzer_innen näherzubringen, ihre Akzeptanz zu fördern und beim effizienten Umgang mit ihnen hilfreich zur Seite zu stehen.

Alle Bibliotheken der Fachbereichsbibliothek werden aufgrund ihres umfangreichen Angebotes in einem hohen Ausmaß auch von externen Benutzer_innen und Wissenschafter_innen aus dem In- und Ausland frequentiert. 
Häufig werden die am Standort angebotenen Services auch sehr unmittelbar benötigt. Das ist unter anderem an den von den Benutzer_innen hinterlassenen Spuren erkennbar, die oft scheinbar von der Grabung direkt in die Bibliothek eilen: kleine Erdklumpen, die von den gut strukturierten Schuhprofilen bröckeln, gehören zu den gewohnten Hinterlassenschaften auch in den Bibliotheksräumlichkeiten ...

Eine Besonderheit des Standortes ist die Kooperation mit der Bibliothek Wien des Österreichischen Archäologischen Instituts / Österreichische Akademie der Wissenschaften (ÖAI). Sie teilt sich mit der Bibliothek Klassische Archäologie die Räumlichkeiten und die bibliothekarische Infrastruktur, der gesamte bibliothekarische Ablauf, von der Literaturbeschaffung über die Systematik der Aufstellung hin zur Benutzer_innenbetreuung erfolgt koordiniert. Auch Änderungen in der Bibliothek werden in Absprache und von beiden Teams gemeinsam durchgeführt. Vor allem in den letzten drei Jahren konnte die diesbezügliche Teamfähigkeit durch zahlreiche Umgestaltungen in der Bibliothek mehrfach unter Beweis gestellt werden - ein Großteil des Buchbestandes ist dabei durch die Hände der Bibliothekar_innen gegangen!

Mit der Integration der ÖAI-Bestandsdaten in den Datenbestand der Universitätsbibliothek Wien im Frühjahr 2016, die nach intensiven Datenbereinigungen und -angleichungen vorgenommen werden konnte, wurde ein weiteres sichtbares Zeichen der guten Zusammenarbeit gesetzt. Bis zu diesem Zeitpunkt war der ÖAI-Bestand ausschließlich über den Verbundkatalog durchsuchbar. Seit der Migration der Daten scheint er auch in usearch auf, und die vorher nicht entlehnbaren Werke sind nun analog zum Bestand der Fachbereichsbibliothek entlehnbar. Für die Nutzer_innen bedeutet das eine enorme Vereinfachung im Umgang mit dem Buchbestand vor Ort.

Natürlich bietet der Standort mit seinen unterschiedlichen Aufgabenfeldern, Ansprechpartnern und Besonderheiten auch viele Herausforderungen. Ein Thema, welches das Geschehen an der Fachbereichsbibliothek aber seit einigen Jahren besonders dominiert, ist das massive Platzproblem. Seit 2016 werden in Ermangelung ausreichender Magazinsflächen am Standort kontinuierlich Teile des Bestandes in ein externes Depot der Universitätsbibliothek ausgelagert. Die effiziente Nutzung der verfügbaren Bibliotheksflächen und die Optimierung des vorhandenen Buchbestandes werden auch in Zukunft im Fokus der Arbeit in den Bibliotheken stehen müssen.

Die weitere Entwicklung der Bibliothek in den kommenden Jahren wird sich an ihrem Selbstverständnis orientieren, mit ihrem Leistungsspektrum Wissenschaft, Lehre und Studium bestmöglich zu unterstützen, sowie an ihrem Anspruch, als zielgerichtet agierende und bestens ausgestattete Bibliothek wahrgenommen zu werden. 
Open-Access-Publikation im Sinne der CC-Lizenz BY-NC-ND 4.0

(c) 2019, Vandenhoeck \& Ruprecht GmbH \& Co. KG, Göttingen ISBN Print: 9783847110989 - ISBN E-Lib: 9783737010986 


\section{Dem Himmel so nah. Die Fachbereichsbibliothek Astronomie}

Die Bestände der Fachbereichsbibliothek Astronomie gehen auf Sammlungen zurück, die der Jesuitenorden in seiner 1733 erbauten Sternwarte in der Wiener Innenstadt an der Ecke Postgasse / Bäckerstraße anzulegen begonnen hatte. Im Jahre 1755 übersiedelte die Universitätssternwarte in ein modernes Observatorium am Dach des neu errichteten Universitätshauptgebäudes am Ignaz-SeipelPlatz. 1874 erfolgte die Grundsteinlegung eines eigenen Sternwarte-Gebäudes in Währing, einem 1892 eingemeindeten Vorort Wiens, die 1883 eröffnet werden konnte. In diesem von den berühmten Architekten Ferdinand Fellner (18471916) und Hermann Helmer (1849-1919) errichteten Bau fand auch die Fachbüchersammlung Platz.

Die längste Zeit als Institutsbibliothek geführt, kam es erst während der Direktion von Maria Seissl zur vollständigen Integration dieser einstigen Institutsbibliothek in die Universitätsbibliothek Wien: 2007/2008 wurde die Bibliothek organisatorisch als Fachbereichsbibliothek der Dienstleistungseinrichtung (DLE) Bibliotheks- und Archivwesen zugeordnet. Die DLE-Leitung investierte sofort Ressourcen, um die Retrokatalogisierung und Neusystematisierung des Magazinbestandes durchzuführen. Damit wurde es möglich, Bestände aus dem Freihandbereich direkt im Magazin einzuordnen und den Bücherbestand als gut sichtbaren Teilbestand der Universitätsbibliothek Wien nachzuweisen. Inzwischen ist der gesamte Bestand von derzeit über 14.800 Bänden, darunter etwa 620 Hochschulschriften, online erfasst.

Herzstück der Bibliothek ist heute ein Raum mit Freihandbereich, Infopult und sechs Arbeitsplätzen. Die dort befindlichen Bücher sind nach systematischer Ordnung aufgestellt, wobei es sich ausschließlich um Literatur ab dem Erscheinungsjahr 1960 handelt. Unmittelbar angrenzend befindet sich ein Raum für die älteren Zeitschriftenjahrgänge, die wiederum alphabetisch geordnet sind. Die Bücher aus den Jahren 1800 bis 1960 werden in einem Magazin aufbewahrt. Die Zahl der laufenden Zeitschriften in gedruckter Form geht stark zurück, da die überwiegende Zahl mittlerweile online angeboten wird. Die formale Erschließung erfolgt mit Unterstützung des Teams Integrierte Medienbearbeitung 
Fachbereichsbibliotheken. Bücher aus dem Magazinbereich können online oder persönlich bestellt werden und werden umgehend ausgehoben.

Eine Besonderheit stellt der umfangreiche Altbestand dar. Dazu zählen etwa 500 Bücher mit dem Erscheinungsdatum vor 1800, darunter fünf Inkunabeln, sowie 56 Werke, die vor dem Jahr 1600 erschienen sind. Mit der Digitalisierung dieses wertvollen Altbestandes wurde im Jahr 2011 begonnen. Viele Titel sind bereits online verfügbar, was einerseits die Benutzung erleichtert und andererseits einen wichtigen Beitrag zur Schonung dieses wertvollen alten Bestandes darstellt. Diese bedeutenden Bücher werden in der ehemaligen Wohnung des Sternwartedirektors, in dem jetzt ein kleines Astronomie-Museum untergebracht ist, in Tresoren gelagert.

Ganz neu sind mehrere Schauvitrinen vor dem Bibliotheksbereich, die nicht nur auf den sehr unauffälligen Eingang zur Bibliothek hinweisen, sondern auch dazu einladen, sich über verschiedene Themen zu informieren, aktuell etwa über das Thema Mond und 50 Jahre Mondlandung. 


\section{Die Universitätsbibliothek kommt ihrem Ziel der Einschichtigkeit wieder ein Stück näher. Die Fachbereichsbibliothek Bildungswissenschaft, Sprachwissenschaft und Vergleichende Literaturwissenschaft}

In den letzten Jahren haben sich im dezentralen Bereich der Universitätsbibliothek Wien einige nennenswerte Entwicklungen ergeben. Unter anderem bekennt sich die Universitätsbibliothek Wien zur Einschichtigkeit. ${ }^{1}$ Neben der Umwandlung des zweischichtigen Systems in ein funktional einschichtiges ist auch die Eingliederung mehrerer kleinerer Teilbibliotheken in größere Einheiten ausdrückliches Ziel der Universitätsbibliothek Wien sowie der Universitätsleitung.

So ist es an der Universitätsbibliothek Wien immer wieder zu Zusammenführungen gekommen. Eine solche führte im Jahr 2010 zur Fachbereichsbibliothek Bildungswissenschaft, Sprachwissenschaft und Vergleichende Literaturwissenschaft. Sie entstand aus der Umwandlung mehrerer Institutsbibliotheken in Fachbereichsbibliotheken und deren Zusammenführung mit zwei weiteren Fachbereichsbibliotheken sowie aus der gleichzeitigen Übersiedlung in einen Neubau in der Sensengasse 3a im 9. Wiener Gemeindebezirk. ${ }^{2}$

Wie kam es zu dieser Zusammenführung? Die Universitäten erhielten mit dem Universitätsorganisationsgesetz 1993 die Teilrechtsfähigkeit. Die Universität Wien zog als letzte Hochschule Österreichs im Jahr 2000 mit dem erklärten

1 Die Einschichtigkeit wird von folgenden Kriterien bestimmt: Einer Bibliotheksdirektion ist das bibliothekarische Personal zugeordnet, eine zentrale Etatverwaltung ist zuständig für die Verteilung des Budgets. In einem zweischichtigen System werden diese beiden Merkmale nicht erfüllt. Keine Unterscheidungszeichen für Ein- beziehungsweise Zweischichtigkeit sind das Vorhandensein mehrerer bibliothekarischer Standorte oder die genaue Ablauforganisation einer Hochschulbibliothek. Vgl. Axel Halle: Zentralisierung und Dezentralisierung. Managementaspekte ein- und zweischichtiger Bibliothekssysteme. In: Bibliothek. Forschung und Praxis 26 (2002), Nr. 1, S. 41-43, hier S. 41.

2 Die Zusammenführung betraf folgende Bibliotheken: die Bibliothek der Forschungseinheit LehrerInnenbildung und Professionalisierungsforschung, die Bibliothek der Forschungseinheit Islamische Religionspädagogik, die Institutsbibliothek Sprachwissenschaft und ihren Teilbereich Indogermanistik, die Bibliothek der Abteilung für Vergleichende Literaturwissenschaft des Instituts für Europäische und Vergleichende Sprach- und Literaturwissenschaft, die Fachbereichsbibliothek Bildungswissenschaft sowie die Fachbereichsbibliothek Sonderund Heilpädagogik. 
Ziel, die bibliothekarischen Standorte zu konsolidieren, nach. Auch 2003 war der von der Universitätsleitung ausgesprochene Auftrag »die Schaffung von größeren bibliothekarischen Einheiten durch Zusammenlegung von Teilbibliotheken und insgesamt die Optimierung des Personaleinsatzes sowie die Bereinigung der Personalsituation in den dezentralen Bibliotheken $\aleph^{3}$.

Da die von der Universität Wien angemieteten Adressen Garnisongasse (Institut für Bildungswissenschaft) und Berggasse (Institut für Sprachwissenschaft und Abteilung für Vergleichende Literaturwissenschaft des Instituts für Europäische und Vergleichende Sprach- und Literaturwissenschaft) aufgrund auslaufender Mietverträge aufgegeben werden mussten, war es naheliegend, dass diese Institute miteinander in den Neubau ziehen sollten. Freilich betraf die Übersiedlung auch alle anderen bildungswissenschaftlichen Institutsteile, die zu diesem Zeitpunkt auf mehrere Standorte verteilt waren. In Fällen der Übersiedlung in einen Neubau gilt die Maxime der Universitätsbibliothek Wien: eine Bibliothek pro Haus. Das Projekt zum Neubau startete für die Universitätsbibliothek im Jahr 2009.

Vonseiten der Universitätsbibliothek wurde auf allergrößte Transparenz zwischen ihr und den betroffenen universitären Einrichtungen geachtet, was in mehreren Treffen und Austauschrunden 2009 und 2010 mündete. Ziel dieser Treffen war es, möglichst viele der Bibliotheksverantwortlichen und Institutsangehörigen zu erreichen, sie zu informieren und ihnen auch für auftauchende Fragen zur Verfügung zu stehen.

Am 28. April 2010 übernahm die Universität Wien das Gebäude vom Bauträger. Anschließend wurde das Objekt für den Universitätsbetrieb eingerichtet und ausgestattet. Die Übersiedlung der einzelnen Teilbibliotheken ging in der Zeit vom 17. August bis 29. September 2010 reibungslos über die Bühne. Die Bibliothek konnte so am 1. Oktober 2010 gemeinsam mit dem Rest des Hauses eröffnet werden. ${ }^{4}$ Sie wies nach der Zusammenlegung einen Bestand von etwa 190.000 Druckschriftenwerken und ca. 300 laufenden Zeitschriftenabonnements auf.

Eine Zusammenführung sehr ähnlicher, einander überschneidender Bereiche war sinnvoll; zwischen manchen der Teilbereiche gab es große Schnittstellen, vor allem was die verschiedenen bildungswissenschaftlichen Teilbibliotheken betraf. Aber auch die sprachwissenschaftlichen Bestände überschnitten sich in manchen Bereichen mit den bildungswissenschaftlichen (Spracherwerb, Mehrsprachigkeit im Bildungswesen, Zweisprachigkeit, bilinguale Schulen,

3 Vgl. Leistungsbericht 2004 der Universitätsbibliothek Wien, S. 13.

4 Siehe auch den Online-Artikel Haus Sensengasse 3 a feierlich eröffnet: http://medienportal.uni vie.ac.at/uniview/studium-lehre/detailansicht/artikel/haus-sensengasse-3a-feierlich-eroeff net/ [Letzter Zugriff: 01.01.2019]. 
Migration und Schule). Zugleich war die Zusammenführung durchaus komplex, handelte es sich doch um sehr unterschiedliche Bibliotheken: von Fachbereichsbibliotheken bis zu Institutsbibliotheken und Teilbibliotheken von Instituten. Somit waren auch die verwendeten Bibliothekssysteme und Titelaufnahmen unterschiedlich.

Natürlich ist eine solche Zusammenführung ein Ereignis, das die betreffenden Mitarbeiter_innen nicht öfter durchmachen möchten. Im Fall der Universitätsbibliothek Wien war es von großem Vorteil, dass das Referat Bauagenden und Bestandserhaltung der Universitätsbibliothek maßgebliche Erfahrungen aus Projekten der vergangenen Jahre einbringen konnte. Mit der Zusammenführung konnten außerdem Stellen bereinigt werden, da bisheriges Institutspersonal von der Universitätsbibliothek übernommen wurde.

Eine Erleichterung stellte die Vergabe der Planung der Bestandsaufstellung als Projekt an ein Team Studierender des Universitätslehrgangs Library and Information Studies dar. Das Projektteam errechnete gemeinsam mit dem Team der Fachbereichsbibliothek die Laufmeter und Regalflächen und erarbeitete die Aufstellung der Bestände. Es wurde beschlossen, die einzelnen Teilbereiche separat aufzustellen und die jeweiligen Aufstellungssysteme beizubehalten. Somit sind manche Bestände nach einer hauseigenen Systematik aufgestellt, andere wiederum nach numerus currens. Der Grund dafür ist mehrschichtig. Zum einen hätte eine komplette systematische Aufstellung viel mehr Platz benötigt. Zum anderen wäre ein groß angelegtes Projekt zur Ausarbeitung einer geeigneten Aufstellungssystematik notwendig gewesen. Es wäre ein Unterfangen von mehrjähriger Dauer gewesen, einen so großen Bestand aufzuarbeiten, neu zu signieren und umzustellen. In der kurzen Zeit vor der Übersiedlung war dies nicht möglich.

Durch die Einbindung in das integrierte Bibliothekssystem Aleph wurden sämtliche Geschäftsgänge vereinheitlicht. Auch die Retrokonversion der Bestände schritt zügig voran, sodass rasch alle Titel in $\mathrm{u}$ :search, der Suchmaschine der Universitätsbibliothek Wien, abrufbar waren, was die teilweise noch vorhandenen Zettelkataloge bald obsolet machte. Es kam außerdem zum ersten Mal $\mathrm{zu}$ einer korrekten Zeitschriftenverwaltung. Mehrfachabonnements wurden abbestellt, und wo es möglich war wurde zur elektronischen Ausgabe gewechselt.

Auch der Weg vom Buchwunsch bis zum bereitgestellten Werk wurde um etliches kürzer als vorher. Eine Kollegin vom Team Integrierte Medienbearbeitung Fachbereichsbibliotheken wurde mit in die neue Bibliothek übersiedelt und kümmerte sich nun um Bestellkatalogisierung, Erwerbung und Titelaufnahme. Somit entfiel auch die Notwendigkeit eines Büchertransports zwischen Fachbereichsbibliothek und Katalogisierungsabteilung. Ebenfalls wurden die Öffnungszeiten großzügig erweitert. So war die neue Fachbereichsbibliothek Bildungswissenschaft, Sprachwissenschaft und Vergleichende Literaturwissen- 
schaft in ihrer Anfangszeit die einzige Fachbereichsbibliothek, die auch samstags geöffnet hatte.

Mit der Einrichtung der neuen Fachbereichsbibliothek Bildungswissenschaft, Sprachwissenschaft und Vergleichende Literaturwissenschaft war die Universitätsbibliothek dem Ziel der Einschichtigkeit wieder ein Stück nähergekommen. Zum einen waren mehrere Institutsbibliotheken in eine Fachbereichsbibliothek umgewandelt worden, zum anderen waren mehrere Teilbibliotheken zusammengelegt worden. Diese neue Bibliothek stand unter einer Leitung und war nicht nur verwaltungstechnisch, sondern auch im Zuge einer Standortreduktion zusammengeführt worden. Somit konnte die Universitätsbibliothek Wien ein weiteres ihrer Projekte in die Tat umsetzen. 


\section{Auch eine Frage des Standortes. Die Geschichte der Fachbereichsbibliothek Biologie}

Die Fachbereichsbibliothek Biologie entstand 1982 durch die Zusammenlegung von drei Institutsbibliotheken - jene des Pflanzenphysiologischen (gegründet 1849), des Zoologischen (gegründet 1896) und des Anthropologischen Instituts (gegründet 1919). Damit wurde ein Bestand von etwa 43.000 Bänden an einem Standort zusammengeführt.

Ursprünglich waren für die Bibliothek Räumlichkeiten im damals neu errichteten Biozentrum im Universitätszentrum Althanstraße (UZA) vorgesehen, sie musste aber aus Platzgründen im benachbarten Gebäude der damaligen Wirtschaftsuniversität Wien untergebracht werden. Zu diesem Zeitpunkt wurde davon ausgegangen, dass die zum Biozentrum gehörende Bibliothek auf Dauer an diesem Standort bleiben würde. Rückblickend betrachtet war dies keine Selbstverständlichkeit - und jetzt sind die Jahre an diesem Standort gezählt.

\section{Brandstiftung}

Ein großer Schlag traf die Fachbereichsbibliothek Biologie, als im Dezember 2005 mehrere Brände in der Wirtschaftsuniversität gelegt wurden und der größte Brandherd in der Fachbereichsbibliothek lag. Zwar wurden die Flammen durch Sauerstoffmangel erstickt, aber der gesamte Literaturbestand, der Lesesaal und die Büroräume waren vollkommen verrußt. In Folge mussten alle Bücher abtransportiert und einer aufwendigen Reinigung durch eine Spezialfirma unterzogen werden. Die genannten Räume wurden generalsaniert und die Mitarbeiter_innen der Bibliothek fanden in der benachbarten Fachbereichsbibliothek Erdwissenschaften und Meteorologie und der Fachbereichsbibliothek Pharmazie und Ernährungswissenschaften Unterkunft. Dort wurde für einige Monate ein Notbetrieb der Fachbereichsbibliothek Biologie eingerichtet, bis sie am 19. Juni 2006 wiedereröffnet wurde. Durch die Neugestaltung der Bibliothek konnten im Zuge der Sanierung viele Verbesserungen erzielt werden, so ist zum Beispiel der wertvolle Buchbestand nun brandsicher untergebracht und der 
Lesesaal geräumiger und heller gestaltet. Neben Computersitzplätzen stehen nun auch Stand-PCs zur Verfügung. Ebenso wurde eine Buchsicherungsanlage installiert und der Gesamtbestand im Zuge der Neuaufstellung magnetisch gesichert.

\section{Umzug der Wirtschaftsuniversität}

Im Sommer 2013 übersiedelte die Wirtschaftsuniversität Wien in den Prater und die Fachbereichsbibliothek Biologie sollte aus dem fast leerstehenden Gebäude ausgesiedelt werden. Es folgte eine Machbarkeitsstudie für die Errichtung einer neuen Bibliothek in den Hörsälen eines anderen Gebäudes des Universitätszentrums Althanstraße, dem sogenannten UZA 3, aus Kostengründen wurde dieser Plan aber verworfen. Die Fachbereichsbibliothek Biologie blieb daher in dem großen, praktisch leeren Gebäude - eine Situation, die von manchen Benutzer_innen als etwas unheimlich angesehen wurde. Mit der Zeit erhielt die Fachbereichsbibliothek aber neue Mitbewohnerinnen: die Universität für Bodenkultur, die Technische Universität Wien und die Universität für Angewandte Kunst nutzten und nutzen die einst leeren Räumlichkeiten als Ausweichquartier.

\section{Neuplanung}

Seit dem Sommer 2016 plant die Fachbereichsbibliothek Biologie eine neue Bibliothek für das Biozentrum St. Marx. Am 19. September 2018 erfolgte der Spatenstich für den Neubau und im Wintersemester 2021/22 soll das Biozentrum mit der Fachbereichsbibliothek am neuen Standort in Betrieb gehen. Mit der Neuerrichtung der Fachbereichsbibliothek ergibt sich die Chance, auf die veränderten Bedürfnisse der Leserschaft und Anforderungen an Bibliotheken einzugehen. So sind etwa sechs Gruppenarbeitsräume für je sieben Personen vorgesehen und neben den Lese- und Rechercheplätzen ist auch ein bibliotheksinterner Computerraum mit 15 Arbeitsplätzen geplant. Dieser Raum wird für Bibliotheksschulungen ausgestattet sein. Da der Bibliotheksbestand neu aufgestellt werden muss - aus Platzgründen wird der Anteil der Kompaktregale stark erhöht - bietet es sich an, die Aufstellungssystematik zu überarbeiten und den neuen Forschungsbereichen anzupassen. Für die Studierenden ergibt sich am neuen Standort eine gute Erreichbarkeit der Bibliothek, da sie - im Gegensatz zum Universitätszentrum Althanstraße - im gleichen Gebäude wie die Institute und noch dazu zentral in der Nähe der Hörsäle gelegen ist. 


\section{Weitere Herausforderungen}

Neben der Standortfrage hat die Fachbereichsbibliothek Biologie auch andere Herausforderungen zu bewältigen. Gerade in den Naturwissenschaften haben sich die elektronischen Medien bereits sehr früh durchgesetzt und so wurden seit 2011 schrittweise die Printversionen der Zeitschriften durch Onlineversionen ersetzt. Derzeit sind in der Biologie physisch nur mehr die nicht elektronisch verfügbaren Zeitschriften aus dem Bereich Fachdidaktik zu finden. Auch haben in den letzten Jahren einige Departments des Biozentrums ihre Forschungsschwerpunkte verlagert und es sind neue Forschungsrichtungen hinzugekommen. Neben der Neuausrichtung auf die Molekularbiologie und Systembiologie ist auch die Mikrobiologie vermehrt in den Fokus der ökologischen Departments gerückt. Zusätzlich übersiedelten 2008 zwei Departments (Division of Tropical Ecology and Animal Biodiversity und Division of Conservation Biology, Vegetation- and Landscape Ecology) an das Botanische Institut am Rennweg. Infolge all dieser Veränderungen kam es bei der Literaturbeschaffung der Bibliothek zu neuen Schwerpunkten. Auf Wunsch konnte erstmals für die Wissenschafter_innen eines Departments eine molekularbiologische Datenbank angekauft werden. Das Biozentrum ist auf den Zugang zu entsprechenden Faktendatenbanken angewiesen und so ist zukünftig mit weiteren Ankaufswünschen zu rechnen.

\section{Fachdidaktische Anlaufstelle}

Der Bereich Fachdidaktik Biologie wurde von der Fachbereichsbibliothek schon immer intensiv betreut, durch eine neue Professur im Sommer 2018 ist sie jetzt aber ebenfalls ein neuer Schwerpunkt. Die Bibliothek hält alle wichtigen österreichischen Schulbücher für den Biologieunterricht und Monographien zur Fachdidaktik sowohl für Lehramtsstudierende als auch für Lehrende bereit. Neuankäufe sind zusätzlich für das Österreichische Kompetenzzentrum für Didaktik der Biologie (AECC Bio) geplant.

Dabei ist die Fachbereichsbibliothek Biologie auch für Schüler_innen eine wichtige Anlaufstelle. Für die verpflichtende Vorwissenschaftliche Arbeit (VWA) - eine Säule der neuen Matura in Österreich - wird oft ein biologisches Thema gewählt, was zu einer Intensivierung der schon lange stattfindenden Zusammenarbeit mit Lehrer_innen führte. Durch Klassenführungen und Schulungen zur Recherche für biologische VWA-Themen kommen Jugendliche oft erstmals in Kontakt mit der Universitätsbibliothek. Dank Freihandaufstellung und übersichtlicher Aufstellungssystematik ist die Fachbereichsbibliothek eine besonders benutzerfreundliche Einrichtung für diese Zielgruppe und andere Ex- 
terne. Bewährt haben sich auch die Führungen und Schulungen für Studierende. Für Studienanfänger_innen buchen oft Mentoren_innen Einführungen, Lehrende organisieren für Fortgeschrittene Schulungen zu Themen wie wissenschaftliches Schreiben oder professionelle Recherche.

\section{Bestmögliches Service}

Die letzten Jahre der Fachbereichsbibliothek Biologie waren sehr spannend und es stehen weitere herausfordernde Jahre bevor. Mit dem Ziel, immer bestmögliche Services zu bieten, reagiert die Fachbereichsbibliothek ständig auf die sich verändernden Bedürfnisse von Lehrenden und Studierenden. So stieg sie im Wintersemester 2018/2019 von Wochenendentlehnung auf 7-Tage-Entlehnung um. Damit konnte dem bei einer Umfrage erhobenen Wunsch nach längeren Entlehnfristen entsprochen werden. Durch den Neubau der Bibliothek im Biozentrum St. Marx ergeben sich weitere Verbesserungsmöglichkeiten. Bis zum geplanten Umzug des Biozentrums im Sommersemester 2021 stehen neben den räumlichen Planungen mit den Architekten vor allem bibliotheksinterne Diskussionen zur Optimierung der neuen Fachbereichsbibliothek auf der Tagesordnung. Bestmögliches Service bleibt weiter unser Ziel: Wir wechseln den Standort, aber nicht unseren Standpunkt. 


\section{Blüten, Bücher, Bytes - Systematische Botanik im digitalen Zeitalter. Die Fachbereichsbibliothek Botanik}

\section{Gründungsgeschichte}

Den Startschuss für die Fachbereichsbibliothek Botanik setzte schon Erzherzogin Maria Theresia im Jahr 1749. Damals wurde an der Universität Wien eine Professur für Botanik und Chemie eingerichtet und ab 1754 der botanische Garten in der damaligen »ungarischen Vorstadt " gestaltet. Eine erste Schenkung von 85 facheinschlägigen Dubletten aus der kaiserlichen Hofbibliothek begründete zeitgleich die botanische Bibliothek am Rennweg. Konsequenter Bestandsaufbau und zahlreiche Schenkungen wertvoller Professoren-Bibliotheken führten dann zu einem raschen Anwachsen. Um 1855 erreichte die Bibliothek einen Bestand von rund 8.000 Bänden zur morphologisch-systematischen Botanik und stand in dem Ruf, sie habe »vielleicht ihres Gleichen in Europa nicht und [sei] ein wahrer Tempel des Studiums der Botanik « ${ }^{1}$.

\section{Eingliederung in die Universitätsbibliothek und der Weg zur Entlehnbibliothek}

Lange Zeit eine reine Institutsbibliothek, erfolgte erst im Zuge des UniversitätsOrganisationsgesetzes 1975 eine organisatorische Trennung von der Forschung und die formale Eingliederung in die Universitätsbibliothek Wien. Am 18. Februar 1990 wurde die Institutsbibliothek dann in eine »Fachbibliothek für Botanik« umgewandelt und Robert Stangl im Juli 1990 als erster offizieller Bibliotheksleiter bestellt. ${ }^{2}$ In seine Dienstzeit fällt auch die Aufstellung des ersten Personal-Computers für Verwaltungs- und Bibliographier-Aufgaben im Winter

\footnotetext{
1 August Neilreich: Geschichte der Botanik in Nieder-Oesterreich. In: Verhandlungen des zoologisch-botanischen Vereins in Wien 5 (1855), S. 23-76, besonders S. 72.

2 Robert Stangl: Die botanische Bibliothek am Rennweg. In: Abhandlungen der ZoologischBotanischen Gesellschaft in Österreich 26 (1992), S. 50-68.
} 
1990 sowie diverse strukturelle Veränderungen: 2005 umfasste das umbenannte »Fakultätszentrum für Biodiversität» nur zwei Abteilungen (»Botanische Systematik und Evolutionsforschung" sowie "Strukturelle und Funktionelle Botanik«), 2008 wurden dann die Bereiche "Naturschutzbiologie, Vegetations- und Landschaftsökologie« sowie »Tropenökologie und Biodiversität der Tiere« vom Biozentrum Althanstraße an den Rennweg transferiert und der Literaturankauf der Bibliothek entsprechend den neuen Departments angepasst. Ein großer Buchbestand, der etwas überhastet mit übersiedelt wurde, konnte von der Fachbereichsbibliothek Botanik erst im Nachhinein gesichtet werden. Nach Aufarbeitung dieser rund 3.500 Bände wurden Dubletten an die Fachbereichsbibliothek Biologie rückgestellt. Seit 2013 ist die Fachbereichsbibliothek Botanik eine Entlehnbibliothek; die Möglichkeit zur Ausleihe bis zu vier Wochen wurde von vielen Studierenden sehr gut angenommen. Gleichzeitig dient sie aber immer noch im Sinne einer »Institutsbibliothek « als Informationsvermittler für die Fachwissenschafter_innen vor Ort.

\section{Neue Entwicklungen und digitale Angebote}

Das Jahr 2014 brachte einerseits die Umbenennung des Standortes in "Department für Botanik und Biodiversitätsforschung « und andererseits die Pensionierung von Robert Stangl im Dezember. Neue Leiterin der Fachbereichsbibliothek Botanik wurde Eva-Maria Mikschi-Marischler, Matthias Svojtka - seit 2004 in steigendem Ausmaß für die Bibliothek tätig - konnte für 30 Wochenstunden angestellt und eine neue geringfügige Stelle für studentische Mitarbeiter_innen geschaffen werden. Ein neues Leitsystem informiert die Benutzer_innen über den schnellsten Weg in die Bibliothek, auch ein Arbeitszimmer und die Zeitschriftenauslage wurden neu adaptiert. Laufend erfolgt, soweit möglich, die Umstellung auf E-Only-Zeitschriften und -Buchserien, neue EJournals wurden und werden angekauft. Besonders positive Synergieeffekte ergeben sich durch die gemeinsame Leitung von Fachbereichsbibliothek Biologie und Fachbereichsbibliothek Botanik: Der Literaturankauf dieser sehr fachverwandten Bibliotheken kann so perfekt abgestimmt werden, "modernere" Forschungsgebiete wie Pflanzenphysiologie, Molekularbiologie und Pflanzengenetik, sowie »Einsteigerliteratur « für Studienanfänger_innen werden dabei von der Fachbereichsbibliothek Biologie abgedeckt. Die Fachbereichsbibliothek Botanik kann sich hingegen ganz auf ihre traditionellen Schwerpunkte Systematik, Morphologie und Geobotanik konzentrieren und hat in diesen Forschungsbereichen vermutlich auch 2018 »ihres Gleichen nicht«. Zusätzlich erweitert sie auch ständig ihr Literaturangebot in den Fachgebieten Palynologie, Entomologie, Tropenbiologie und Geschichte der Biowissenschaften. Ausgehend vom 
»Europeana Libraries Project« im Jahr 2012 digitalisiert die Fachbereichsbibliothek Botanik darüber hinaus laufend ausgewählte seltene und wichtige urheberrechtsfreie Literatur. 59 »Kronjuwelen« konnten dem Europeana-Projekt inklusive umfangreicher beschreibender Metadaten beigesteuert werden, derzeit (Stand: 14. 12. 2018) umfasst die PHAIDRA-Collection »Botanik (o:167308) 726 Titel. Dabei werden alle Arbeitsschritte der Erstellung eines E-Books, von der Katalogisierung über die Digitalisierung bis hin zum Hochladen des digitalen Objekts in PHAIDRA mit dem Book-Importer, an der Fachbereichsbibliothek Botanik ohne Hilfe anderer Abteilungen durchgeführt. Der ursprünglich geleaste »Bookeye 4«-Produktionsscanner konnte im Jahr 2017 schlussendlich angekauft werden, da die Digitalisierung von einem Projekt in den Regelbetrieb überging. Seit Februar 2015 werden besonders interessante Titel als »E-Book des Monats« auf der Homepage der Bibliothek vorgestellt. Weil die systematische Botanik, anders als viele andere Bereiche der Naturwissenschaften, alte Literatur für den täglichen Forschungsbetrieb dringend benötigt, wurden auch zunehmend bereits existierende Katalogisate mit Volltext-Links (beispielsweise aus der Biodiversity Heritage Library oder der Bayerischen Staatsbibliothek) angereichert. Zum Stand von Dezember 2018 können so zusätzlich rund 3.500 Titel direkt über den Katalog angesteuert und gelesen werden.

\section{Historische Sammlung und Ausblick}

Im Juli 2013 kam es zur formalen »Gründung« der Historischen Sammlung des Departments für Botanik und Biodiversitätsforschung für bis dahin im Sperrmagazin besonders geschützt verwahrte Objekte. Durch laufende Übergaben aus dem Haus mehrt sich seither dieser Sammlungsbestand unter der Leitung von Matthias Svojtka stetig. So konnte beispielsweise die gesamte historische Diaund Fotosammlung (rund 150 Schachteln und zwei mit Glasdias gefüllte Schränke) gesichert und neu aufgestellt werden - auch mit der Digitalisierung historischer Objekte wurde bereits begonnen. ${ }^{3}$ Seit einem (nicht erfolgreichen) Einbruch in die Bibliothek im Jahr 2014 verfügt das Sperrmagazin über eine Sicherheitstür. Die räumliche und personelle Einheit von Sammlung und Bibliothek ergibt wiederum äußerst positive Synergieeffekte: So wurde beispielsweise die Ausstellung zum 650-Jahr-Jubiläum der Universität Wien Das Wissen der Dinge mit Objekten aus den Bereichen Bibliothek und Sammlung bestückt, die wunderbaren Blütenmodelle von Robert Brendel (ca. 1821-1898) bildeten sogar das Motiv der offiziellen Einladung zur Ausstellungseröffnung. Auch bei bisher zwei Besuchen der Gregor Mendel-Gesellschaft ergänzten sich Beson-

3 PHAIDRA Collection »Historische Objekte Botanik«, https://phaidra.univie.ac.at/o:292193. 
derheiten von Fachbereichsbibliothek und Sammlung perfekt: Der SeparatDruck der Versuche über Pflanzen-Hybriden von Gregor Mendel (1822-1884) aus dem Jahr 1866 entwickelt neben der Portrait-Fotographie von Mendels Schwester Theresia, verheiratete Schindler (1829-1908), eine ganz eigene historische Dynamik. Beide liegen übrigens in digitaler Form vor. ${ }^{4}$ Für die Zukunft plant die Fachbereichsbibliothek Botanik eine noch intensivere Bestandserschließung, beispielsweise durch die Katalogisierung der umfangreichen Sonderdruck-Sammlung (rund 900 Kartons), wie auch eine erhöhte Sichtbar-Machung der Bestände durch eigene Digitalisierung sowie Verlinkung mit bereits bestehenden Ressourcen. Unsere Forschungsobjekte, die Pflanzen, und das Wissen über sie in all seinen Repräsentationen können so in veränderter, digitaler Form neu erblühen und eröffnen eine Perspektive auf die kommenden 250 Jahre Bibliotheksgeschichte.

4 https://phaidra.univie.ac.at/o:171522 und https://phaidra.univie.ac.at/o:293760. 


\section{Die neue Bibliothek in der Alten Universität. Die Fachbereichsbibliothek Byzantinistik und Neogräzistik}

Eine der vielen Neuerungen in der Amtszeit von Maria Seissl betraf die Fachbereichsbibliothek Byzantinistik und Neogräzistik, die durch eine räumliche Erweiterung und Umgestaltung eine bedeutende positive Veränderung erfuhr:

Im Jahr 2008 gehörte die Fachbereichsbibliothek Byzantinistik und Neogräzistik aufgrund des seit über einem Jahrzehnt ungelösten akuten Platzmangels zu den langjährigen »Problemfällen« innerhalb der Universitätsbibliothek Wien, deren Betrieb nur durch die alte österreichische Kulturtechnik des »Durchwurstelns « aufrechterhalten werden konnte. Denn die Fachbereichsbibliothek in den Räumlichkeiten des Instituts für Byzantinistik und Neogräzistik, das sich seit 1975 im Alten Universitätsgebäude in der Postgasse befindet, war im wahrsten Sinne des Wortes "voll«. Die Bibliotheksbestände verteilten sich bereits über sämtliche Räume des Instituts, Büros von Mitarbeiter_innen und Hörsaal eingeschlossen. In den ursprünglichen Bibliotheksräumlichkeiten waren die Regale bis zu einer Höhe von zehn Fachböden eng mit Büchern vollgestellt und konnten nur mithilfe von wenig vertrauenserweckend wirkenden Hängeleitern erreicht werden. Auch in den beiden Leseräumen waren die Regale bis an die Decke mit Büchern befüllt. Benutzer_innen mussten einen regelrechten Parcours absolvieren, bis sie das gewünschte Buch aus der angeblichen »Freihandaufstellung" schließlich in Händen hielten - nicht selten begleitet von unwirschen Kommentaren von Institutsmitarbeiter_innen oder Doktorand_innen, die vom ständigen Leitern-Verschieben hinter ihren Schreibtischen enerviert waren. Erschwerend kam hinzu, dass aufgrund des Platzmangels immer wieder Teilbestände an andere Orte, wo es eben gerade noch etwas freien Platz gab, ausgelagert werden mussten, sodass die Aufstellung alles andere als logisch war und viele Bestände nur mit der Hilfe des Bibliothekspersonals überhaupt aufzufinden waren. Doch selbst letzterem gelang es kaum, den Überblick zu behalten. 


\section{Eine Bibliothek von internationalem Ruf}

Die Fachbereichsbibliothek Byzantinistik und Neogräzistik ist eine der renommiertesten Bibliotheken ihres Faches, die regelmäßig von Wissenschafter_innen aus der ganzen Welt für Forschungsaufenthalte besucht wird und deren Neuerwerbungsliste anderen Bibliotheken dieses Faches weltweit zur Orientierung dient. Die Bibliothek ist traditionell eng mit dem Institut für Byzantinistik und Neogräzistik der Universität Wien verbunden und auch die Bibliotheksangehörigen waren stets wissenschaftlich aktiv: Zunächst wurde die Bibliothek von den späteren Universitätsprofessoren Johannes Koder, Wolfram Hörandner und Otto Kresten betreut, der erste Fachbereichsbibliotheksleiter innerhalb der Universitätsbibliothek Wien, Ernst Gamillscheg, wurde später Leiter der Handschriftenabteilung der Österreichischen Nationalbibliothek. Sein Nachfolger Michael Grünbart ist heute Universitätsprofessor für Byzantinistik an der Universität Münster. Die derzeitige Leiterin der Fachbereichsbibliothek und Autorin dieses Beitrags hat 2018 ihre Dissertation zur Geschichte der Griechen in Wien publiziert. In Anbetracht der wissenschaftlichen Bedeutung der Bibliothek war es umso bedauerlicher, dass ihre Benutzerfreundlichkeit aufgrund der beschriebenen Zustände als äußerst gering zu bezeichnen war.

\section{Die langersehnte Übersiedlung}

Im Jahr 2011 konnte endlich die seit langem gewünschte positive Veränderung in die Wege geleitet werden. Immer wieder hatte es vonseiten des Instituts Versuche gegeben, eine angrenzende, seit Jahren leerstehende große Räumlichkeit - in der sich zuvor das Passamtsarchiv der Polizei befunden hatte - für das Institut zu gewinnen. Die Bemühungen waren jedoch ein ums andere Mal gescheitert. Im Jahr 2011 ergab sich eine einmalige, günstige Gelegenheit, das Raumproblem in der Postgasse einem positiven Ende zuzuführen, das aber ohne das große Engagement aller Beteiligten (ganz besonders der damaligen Institutsvorständin Maria A. Stassinopoulou, Wolfgang Nikolaus Rappert, Christian Posch und Petra Greger) wahrscheinlich ungenützt verstrichen wäre. Noch zu dem Zeitpunkt, als bereits alle Referenzwerke der Fachbereichsbibliothek aus dem sogenannten Katalograum vorübergehend in den Hörsaal des Instituts transferiert worden waren, stand das Projekt kurz davor, doch noch zu scheitern. Wie die Erfahrung leider gezeigt hat, können solche begonnenen und abgebrochenen Umbauprojekte die Situation sogar weiter verschlechtern. Von diesem Schicksal blieb die Fachbereichsbibliothek Byzantinistik und Neogräzistik aber glücklicherweise verschont und im Februar 2012 konnte die Übersiedlung der Bücher in den neu 
adaptierten Raum zwischen Institut für Byzantinistik und Neogräzistik und Universitätsarchiv stattfinden.

\section{Die jetzige Situation}

In der neuen Freihandaufstellung, die nun dieser Bezeichnung tatsächlich gerecht wird, konnten die Bücher endlich wieder in der Reihenfolge der bibliothekseigenen Systematik aufgestellt werden und sind jetzt für die Benutzer_innen leicht zugänglich, was sich sogleich in einer gesteigerten Frequenz bemerkbar machte. In der Tat hatte der alte Zustand bei den Studierenden zu einem gewissen Ausweichverhalten in Bezug auf die Benutzung der Bibliothek geführt, was die Autorin aus eigener Erfahrung bestätigen kann. Auch die Vereinheitlichung der Entlehnbedingungen in der Universitätsbibliothek und die damit einhergehende Einführung der 7-Tage-Entlehnung, die einen intransparenten Zustand beendeten - bei dem diejenigen Benutzer_innen, die sich trauten, nach Sondergenehmigungen $\mathrm{zu}$ fragen, bevorzugt wurden - fanden mehrheitlich positive Aufnahme. Des Weiteren konnte die Retrokatalogisierung abgeschlossen werden, sodass der Gesamtbestand der Fachbereichsbibliothek inklusive aller Zeitschriften-Holdings heute im Online-Katalog nachgewiesen ist.

Der neue Lesesaal besticht nicht nur durch eine schöne Aussicht auf die Dominikanerkirche und die Schönlaterngasse, sondern aufgrund der Lage des gleich angeschlossenen Büros der Fachbereichsbibliothek wurde der seit jeher intensive Kontakt der Forscher_innen und Studierenden zu den Bibliotheksmitarbeiter_innen noch einfacher.

Obwohl das Institut für Byzantinistik und Neogräzistik und die Fachbereichsbibliothek weiterhin durch die bauliche Besonderheit des Alten Universitätsgebäudes geprägt sind und daher ihre Eigenheiten aufweisen, hat die Bibliothek durch die Neugestaltung enorm gewonnen. Auch nachdem der neue Zustand bereits zur Normalität geworden ist, zeigen sich ausländische Gäste, die schon länger nicht mehr in Wien waren, durchwegs begeistert von den neuen Bibliotheksräumlichkeiten. Somit wird die Fachbereichsbibliothek Byzantinistik und Neogräzistik ihrem großen fachlichen Renommee heute auch durch eine ansprechende, benutzerfreundliche und von Offenheit gekennzeichnete Umgebung gerecht. 
Open-Access-Publikation im Sinne der CC-Lizenz BY-NC-ND 4.0

(c) 2019, Vandenhoeck \& Ruprecht GmbH \& Co. KG, Göttingen ISBN Print: 9783847110989 - ISBN E-Lib: 9783737010986 


\section{Millionen Jahre Erdgeschichte. Die Fachbereichsbibliothek Erdwissenschaften und Meteorologie}

Im Jahr 1995 entstand die Fachbereichsbibliothek Erdwissenschaften als Freihandbibliothek im Zwischentrakt des neugebauten erdwissenschaftlichen Zentrums und des Pharmaziezentrums aus den Institutsbibliotheken folgender Institute: Geologie, Petrologie, Geochemie (daraus entstanden die heutigen Abteilungen: Department für Geodynamik und Sedimentologie, Department für Lithosphärenforschung und Department für Umweltgeowissenschaften), Mineralogie und Kristallographie sowie Paläontologie.

2006 wurde darüber hinaus der Literaturbestand des Instituts für Meteorologie und Geophysik, der sich nach der Übersiedlung des Institutes größtenteils noch am alten Standort auf der Hohen Warte in den Räumen der Zentralanstalt für Meteorologie und Geodynamik befand, in die Fachbereichsbibliothek integriert, die ab diesem Zeitpunkt die erweiterte Namensbezeichnung Erdwissenschaften und Meteorologie erhielt.

Mit der Fachbereichsbibliothek Pharmazie und Ernährungswissenschaften besteht eine enge Zusammenarbeit. Dies ist durch die räumliche Verbindung der beiden Fachbereichsbibliotheken mit gemeinsamem Eingang, gemeinsamem Informationspult und identischen Öffnungszeiten gegeben. Die Fachbereichsbibliothek Pharmazie und Ernährungswissenschaften befindet sich auf Ebene 4, über eine Treppe innerhalb dieser Fachbereichsbibliothek ist die Fachbereichsbibliothek Erdwissenschaften und Meteorologie, die sich auf Ebene 5 befindet, erreichbar. Zusätzlich ist die Benutzung eines Liftes möglich, wodurch auch ein barrierefreier Zugang gegeben ist. In der Bibliothek befinden sich 45 Benutzerarbeitsplätze. Mit Ausnahme des gesperrten Regals ist der gesamte Bereich für die Benutzer_innen zugänglich, es ist kein Magazin vorhanden. Es handelt sich um eine Präsenzbibliothek mit Wochenendentlehnung. Auch auf Ebene 5 wurde ein Informationspult errichtet, es dient einem Kollegen als Arbeitsplatz, wodurch während der Dienstzeit den Benutzer_innen eine zusätzliche Informationsmöglichkeit geboten wird, ohne dass sie dafür das Stockwerk verlassen müssen. Die Arbeitsräume der Mitarbeiter_innen beider Fachbe- 
reichsbibliotheken befinden sich auf Ebene 4. Es arbeiten zwei Bibliothekare an der Fachbereichsbibliothek.

Von Dezember 2005 bis Juni 2006 waren die Kolleg_innen der Fachbereichsbibliothek Biologie, die nach einem Brandanschlag ihre Arbeitsräumlichkeiten verlassen mussten, in den Räumlichkeiten dieser beiden Fachbereichsbibliotheken untergebracht. Wegen dem nun höheren Benutzeraufkommen wurden die Öffnungszeiten verlängert, was nach deren Auszug beibehalten wurde. Die zusätzlichen Schalterdienste werden seit dieser Zeit durch studentische Mitarbeiter_innen abgedeckt. Die Benutzer_innenzahlen sind stark steigend, waren es im Jahr 200514.700 (gemeinsam mit der Fachbereichsbibliothek Pharmazie), so haben im Jahr 2018 bereits über 41.000 Personen die beiden Fachbereichsbibliotheken aufgesucht. Neben Student_innen der Erdwissenschaften und Meteorologie benutzen auf Grund von vorhandener fachübergreifender Literatur vor allem Biologen, Geographen und Studierende der Universität für Bodenkultur die Fachbereichsbibliothek.

Die Bibliothek besitzt über 83.000 Druckschriftenbände, davon 2.200 Hochschulschriften. Die gedruckten Zeitschriften sind alphabetisch geordnet, wobei sich die Hefte des laufenden Jahrganges in einer Zeitschriftenauslage befinden, die älteren Jahrgänge sind in einer Kompaktanlage aufgestellt. Heute ist die Zahl der gedruckten Zeitschriften stark rückläufig zu Gunsten der Online-Ausgaben. Die Bücher des Fachbereichs Erdwissenschaften sind systematisch geordnet und auf zwei Regalbereiche aufgeteilt. Dies war aus Platzgründen notwendig; im einen sind Werke mit Erscheinungsjahr vor 1960 aufgestellt, im anderen die später erschienenen. Der Aufstellungsort ist aus der Signatur ersichtlich. Die später hinzugekommenen Werke der Meteorologie befinden sich an einem eigenen Standort innerhalb der Bibliothek und die vorhandene Ordnung nach numerus currens wurde beibehalten. Der gesamte Buchbestand ist bereits online erfasst. Bei der formalen Erschließung wird das Team der Fachbereichsbibliothek von den Kolleg_innen des Teams Integrierte Medienbearbeitung Fachbereichsbibliotheken unterstützt. Zum Bestandsaufbau trug lange Zeit auch der Tausch je einer geologischen und einer paläontologischen zeitschriftenartigen Reihe bei, die vom jeweiligen Institut an der Universität Wien herausgegeben wurden. Diese Tradition ging schon auf die Zeit der Institutsbibliotheken zurück und wurde bei Gründung der Fachbereichsbibliothek übernommen. Es handelte sich dabei um Reihen und Zeitschriften anderer erdwissenschaftlicher Institutionen, die dadurch erworben wurden. So wurde mit über 50 wissenschaftlichen Instituten aus 18 Ländern ein Schriftentausch durchgeführt. Da aber nach und nach viele dieser Reihen eingestellt und keine neuen Tauschpartnerschaften abgeschlossen wurden, nahm die Zahl der getauschten Schriften immer stärker $a b$, bis vor einigen Jahren die beiden Institute ebenfalls aus Kostengründen die 
Herausgabe ihrer Schriftenreihen einstellten und damit die Erwerbung durch Tausch ein Ende fand.

Abgesehen von den Büchern und Zeitschriften besitzt die Fachbereichsbibliothek auch 6.100 geologische Karten in entsprechenden Spezialschränken. Es sind auch mehrere extragroße Arbeitstische zum Auflegen der Karten vorhanden. Weiters befindet sich die Bibliothek der Geologischen Gesellschaft in den Räumlichkeiten der Fachbereichsbibliothek. Hierbei handelt es sich überwiegend um Jahrbücher, Mitteilungen etc. diverser geowissenschaftlicher Institute, oftmals Literatur, an die sehr schwer heranzukommen ist. Diese Bibliothek innerhalb der Fachbereichsbibliothek wird von Wissenschafter_innen und Student_innen des Departments für Geodynamik und Sedimentologie betreut.

Es besteht eine gute Zusammenarbeit mit den Bibliotheken des Naturhistorischen Museums, vor allem mit der Bibliothek der geologisch-paläontologischen Abteilung. Weiters gibt es eine intensive Kooperation mit der Bibliothek der Geologischen Bundesanstalt. Damit ist die Fachbereichsbibliothek Erdwissenschaften und Meterologie sehr gut mit der wissenschaftlichen Community vernetzt. 
Open-Access-Publikation im Sinne der CC-Lizenz BY-NC-ND 4.0

(c) 2019, Vandenhoeck \& Ruprecht GmbH \& Co. KG, Göttingen ISBN Print: 9783847110989 - ISBN E-Lib: 9783737010986 


\section{War das Karteikärtchen weiblich, weil auch »dienlich «? Von der Zettelwirtschaft an der Fachbereichsbibliothek Europäische Ethnologie}

Betreten langjährige Nutzerinnen und Nutzer den Lesesaal der Bibliothek, fällt ihnen mit Sicherheit auf, dass die beiden großen Karteikästen mit dem Schlagwortzettelkatalog nicht mehr vorhanden sind. Seit der Wiedererrichtung des Instituts für Volkskunde bzw. seinem Einzug in den Hanuschhof im Jahr 1965 wurde die Bibliothek anfänglich von Helmut, dem damals einzigen Assistenten, betreut und wies ihre aufgestellten Werke in Katalogen nach - geordnet nach Autoren in dem einen und nach Schlagworten in dem zweiten. Diese Zettelkataloge, eine Errungenschaft des 19. Jahrhunderts, die ausgehend von den USA die bis dahin vorherrschenden Bandkataloge langsam auch in Europas Bibliotheken ablösten, waren lange Zeit das Findmittel für nach geeigneter Literatur Suchende. So gesehen stellen diese zum Teil sehr aufwändig gefertigten hölzernen Kasten- und Ladenelemente zur Aufbewahrung der schon auf das internationale Bibliotheksformat genormten Karten historische Funktionsträger dar, weswegen der alte Autorenkatalog - quasi als museales und Bibliotheksgeschichte widerspiegelndes Objekt - an der Fachbereichsbibliothek Europäische Ethnologie verblieb. Zählten die Literaturauswahl, der Literaturankauf, die Systematisierung - gleichsam der Aufbau - zu den Agenden von Helmut, so oblag der administrative Teil - sozusagen die Ordnung - der ab 1967 dem Institut zur Verwaltung zugewiesenen Sekretärin Julie. Die Zuständigkeiten innerhalb der bibliothekarischen Arbeiten waren somit ausgehandelt und der Bearbeitungsprozess der Karten der bereits auf einer elektromechanischen Schreibmaschine tippenden Sekretärin überlassen. Die ausschließlich von Frauenhänden über die Walze der Schreibmaschine gezogenen Karten wurden mit Hilfe eines zwischen Drucktype und Papier liegenden Farbbandes nach Betätigung

1 Um dem Genre eines Essays zur Alltagsgeschichte eher zu entsprechen, wurde bewusst auf die Nennung der Nachnamen verzichtet; diese sind nach $\mathrm{zu}$ vollziehen in: Susanne Wicha: »Buchstellagen nach Raumgröße [...] eventuell eine Bücherleiter ...«. Von der volkskundlichen Seminarbücherei zur Fachbereichsbibliothek für Europäische Ethnologie. In: Herbert Nikitsch u.a. (Hg.): Hanuschgasse 3. 50 Jahre Institut für Europäische Ethnologie (= Veröffentlichungen des Instituts für Europäische Ethnologie, 38). Wien 2015, S. 79-113. 
der entsprechenden Druckknöpfe der Tastatur fein säuberlich auf ihrer Vorderseite mit den gewünschten bibliographischen Angaben, den Schlagworten und der Signatur beschriftet und schlussendlich an ihrem entsprechenden Platz der Schubladen des Karteikastens einsortiert.

Julie trat mit dieser weiblichen Tätigkeit in den 1970er Jahren einerseits in die Fußstapfen von Li, die nicht nur während der Lehrveranstaltungen den Projektor für ihren professoralen Ehemann Richard bediente, sondern hilfreich Karteikarten für seine eigene Literatursammlung sowie anfänglich auch für die Bibliothek des Instituts für germanisch-deutsche Volkskunde (1942-1945) anlegte, und andererseits ebenso in die von Luise, in deren Zuständigkeit als wissenschaftliche Hilfskraft die Bearbeitung der Karteikarten ebenfalls fiel. Abgelöst wurde sie von Inge, einer promovierten Germanistin, die Richard 1942 als Verwaltungsassistentin für Institut und Bibliothek zugewiesen worden war. Aus dieser Zeit stammen die damals hergestellten Kataloge in Form schwarzer, mit Deckeln verschließbarer Kartons, befüllt mit beige-orangefarbigen Karteikarten in der Größe von 19 x 14,8 cm (Autorenkatalog) bzw. 9,5 x 7,2 cm (Sachgruppenkatalog). Im Ausarbeitungsprozess waren sie allerdings noch mit einer mechanischen Schreibmaschine bedruckt worden, die kleineren Zettel jeweils mit Durchschlag hergestellt, da oft zwei für das von Richard erstellte elfteilige Sachgruppenverzeichnis von Nöten waren. Wie im Sachgruppenkatalog festgelegt, erfolgte damit kongruierend auch die Aufstellung der Werke in den Buchregalen. Diese aufgenommenen 2.545 Bände bildeten hernach den Grundstock der Bibliothek des Instituts, das von 1964 bis 1972 wieder unter der Leitung von Richard stand.

Anstelle des eingangs erwähnten Helmut führten in der Folge zuerst Olaf und dann, in den 1970er Jahren, Editha und noch später Gertraud lange Jahre den Part der Auswahl der Literatur weiter und übernahmen zudem das inhaltliche Erschließen der aufgenommenen Werke; für kurze Zeit erfolgte diese Beschlagwortung durch Reinhard. Workflowmäßig sah dies so aus, dass die Schlagwörter bzw. Schlagwortketten handschriftlich auf der Hinterseite der Karteikarte vermerkt und mit den entsprechenden Werken zur Adjustierung weitergeleitet wurden. Diese sowie das Tippen der Karteikarten übernahmen abermals und auch weiterhin die Sekretärinnen, im Jahr 1976 Silvia und ab Ende 1977 Franziska. In ihren Arbeitsplatzbeschreibungen manifestierte sich diese bibliothekarische Tätigkeit mit $15 \%$ der Gesamtarbeitszeit, was umgerechnet etwa drei Wochenstunden an Tipp- und Sortierarbeit bedeutete, mussten doch bei einem Autoreneintrag oft eine größere Menge an Schlagwortkärtchen in eine etwas abgewandelte Form der »Preußischen Instruktionen" eingeordnet werden. Zudem erfolgte eine formale und inhaltliche Erschließung ausgesuchter Aufsätze von Volkskundlern und Volkskundlerinnen in Sammelwerken und 
Zeitschriften, die gleichfalls in das eigene Regelwerk eingearbeitet werden mussten.

Obige Arbeitszeitregelung galt in weiterer Folge bis 1992 ebenso für Ilse, die von 1980 bis 2004 am Institut und in der Bibliothek als Sekretärin für Verwaltungsagenden tätig war und in dieser Zeit hunderte Kärtchen - auch an den Wochenenden - durch die Schreibmaschine jagte, immer darauf bedacht, keine Eselsöhrchen beim Einziehen der Zettel zu fabrizieren. Solche Knicke sollten in den Schubladen der Kataloge tunlichst vermieden werden, hinderten sie doch bei der haptischen Suche das schnelle Bewegen der Kärtchen. Beim Einordnen der Katalogkarten stand Ilse auch Jörg zur Seite, der, grafisch sehr versiert, die Karteireiter in den einzelnen Schubladen gestaltete. Diese Reiter brachten mehr Übersicht in die mit Kärtchen vollgefüllten Laden und ermöglichten so einen schnelleren Zugriff auf die zu durchsuchende Kartengruppe.

Die 1990er Jahre brachten einerseits mit dem Beginn der digitalen Revolution einen Umbruch in der Informationsvermittlung, andererseits mit der Umwandlung der Instituts- zur Fachbereichsbibliothek sowie der Zuweisung eines Dienstpostens für diese auch in der Hanuschgasse eine entscheidende Wendung. Seit dem Jahr 1992 wurden Neuerwerbungen der Bibliothek in das jeweilige EDV-System der Universitätsbibliothek Wien eingespeist, die Fortführung der alten Zettelkataloge nur noch bis Ende des Jahres 1994 - es ist dies überdies das Jahr der Umbenennung des Instituts in Europäische Ethnologie - beibehalten. Zudem erfolgte sukzessive die Rückerfassung des gesamten Altbestandes an Büchern und Zeitschriften, sodass seit 2008 der gesamte Bestand online abrufbar ist. Im Übrigen hatte die fortschreitende Digitalisierung von Zeitschriften ebenso die der Kulturwissenschaften - die Beschlagwortung einzelner Zeitschriftenartikel sowie Beiträge aus Sammelwerken hinfällig werden lassen.

Die lange dienliche, gleichsam eine hierarchische Arbeitsteilung und geschlechtsspezifische Zuordnung widerspiegelnde Karteikarte hatte nun auch in der Fachbereichsbibliothek Europäische Ethnologie ihre Existenzberechtigung verloren, die Recherche in den alten Holzladen der Kataloge war obsolet geworden. Ab und zu trifft man noch auf nicht ganz EDV-affine Suchende, die, in der Hoffnung, doch die richtige Literatur zu finden, den alten Autorenkatalog durchsuchen; der alte Schlagwortkatalog jedoch war an sein Ende gekommen. Nur ein paar wenige Karteikärtchen dieser Spezies konnten doch noch eine tragende Rolle übernehmen: Sie fanden ihren Platz als Stütze unter den Stehern eines wackeligen Bücherregals im Arbeitszimmer der Leiterin der Fachbereichsbibliothek und sind damit der Bibliothek immer noch dienlich. 
Open-Access-Publikation im Sinne der CC-Lizenz BY-NC-ND 4.0

(c) 2019, Vandenhoeck \& Ruprecht GmbH \& Co. KG, Göttingen ISBN Print: 9783847110989 - ISBN E-Lib: 9783737010986 


\section{Retro-Charme und neue Maßstäbe. Der Fachbereichsbibliothek Geographie und Regionalforschung in die Karten geschaut}

Die Fachbereichsbibliothek Geographie und Regionalforschung kann auf eine lange Geschichte zurückblicken. Auf Betreiben des Gletscherforschers Friedrich Simony (1813-1896) kam es 1851 zur Errichtung eines Lehrstuhls für Geographie. Simony überließ dem Institut nach seiner Emeritierung 1885 einen Teil seiner Privatbibliothek, welche mit der Bücher- und Kartensammlung des Vereins der Geographen an der Universität Wien den Grundstock der heutigen Fachbereichsbibliothek Geographie bildet.

Die Fachbereichsbibliothek Geographie befindet sich im 5. Stock des 1962 eröffneten Neuen Institutsgebäudes (NIG) der Universität Wien. Ende der 1990er Jahre wurde der Bibliotheksbereich zwar neu strukturiert, die kleinräumige Aufteilung und die in die Jahre gekommene Bibliothekseinrichtung versprühen mittlerweile aber einen gewissen Retro-Charme. 2015 gab die Direktion grünes Licht für die Modernisierung des Gruppenarbeitsraumes, der den rund 3.000 Geographie-Studierenden eine adäquate Arbeitsumgebung mit modernen Seminartischen, versenkbaren Steckdosenboxen, USB-Anschlüssen, zehn Wandsteckdosen und einem qualitativ hochwertigen Buchscanner bietet.

\section{Spatial turn meets Digital turn}

Das bibliotheksweite Retrokatalogisierungsprojekt des Buchbestandes hat seit 2010 manch unikale und wertvolle Bestände zu Tage gefördert und über die Suchmaschine der Universitätsbibliothek Wien u:search für alle transparent gemacht. Ein Sorgenkind, oder besser gesagt, einen Rohdiamanten gab es aber noch: die Kartensammlung der Fachbereichsbibliothek Geographie, seit Sammlungsbeginn Mitte des 19. Jahrhunderts auf geschätzte 120.000 Kartenblätter angewachsen. ${ }^{1}$ Aus der einstigen Gebrauchs- und Lehrmittelsammlung

1 Die Sammlung enthält Karten aus aller Welt, der Schwerpunkt liegt auf dem europäischen Raum (hier v. a. Österreich bzw. Länder der Donaumonarchie). Die älteste Karte ist von 1656, 
war längst eine Sammlung von kulturhistorischem Wert geworden, die sich bis vor ein paar Jahren noch in einem Dornröschenschlaf befand. Die Bestände waren nur über einen lückenhaften Zettelkatalog und interne Verzeichnisse nachgewiesen, in den vielen Kartenschränken hatte sich über die Jahre und Jahrzehnte eine "unbibliothekarische» Unordnung breitgemacht, von einer sachgemäßen Lagerung der zum Teil wertvollen Bestände ganz zu schweigen. Ein Projektauftrag der Direktion im Jahr 2013 eröffnete die große Chance, den unscheinbaren Rohdiamanten endlich zum Funkeln zu bringen.

Das Projekt »UB-Maps« setzte sich zum Ziel, das umfangreiche Kartenmaterial der Fachbereichsbibliothek zu katalogisieren (und damit erstmals für alle sichtbar zu machen) und die urheberrechtsfreien Karten digital - und somit zeitund ortsunabhängig - zugänglich zu machen. Die digitalen Objekte werden im gesamtuniversitären Digital-Asset-Management-System PHAIDRA mit einem permanenten Link dauerhaft archiviert. ${ }^{2}$

Als erste Bibliothek in Österreich wurden im Zuge der Katalogisierung die geographischen Koordinaten mit Hilfe einer Bounding Box erfasst (Geotagging) und die Metadaten damit angereichert. Urheberrechtsfreie Karten werden nach der Formalerschließung mit einer Auflösung von 600 dpi und einer 24-BitFarbtiefe inhouse gescannt ${ }^{3}$ und über eine Website der Universitätsbibliothek Wien präsentiert. ${ }^{4}$ Der Goobi viewer gewährleistet eine optimale Anzeige und Darstellung der digitalisierten Karten durch Funktionalitäten wie konfigurierbarem Zoom mit verlustfreiem Skalieren, Panning oder Anzeige von Thumbnails. Ein Permalink (URN) sorgt für die dauerhafte Identifizierung und die zuverlässige Zitierbarkeit, die Verlinkung zur Bibliotheks-Suchmaschine $\mathrm{u}$ :search eröffnet weitere Optionen.

Mit diesem Projekt setzt die Universitätsbibliothek Wien zudem wichtige konservatorische Schritte: Einerseits wird der Zugriff auf die wertvollen und empfindlichen Originalmaterialien durch die digitale Bereitstellung verringert, andererseits bleiben die Karten auch für künftige Generationen durch Umlagerung in säurefreie Mappen erhalten.

Es steckt noch viel Potential im Projekt »UB-Maps«, beispielsweise die Implementierung einer geographischen Bereichssuche oder eine Georeferenzierung 2.0, aber dies steht (noch) auf einem anderen (Karten-)Blatt ...

der Großteil der Karten stammt aus dem 19. und 20. Jahrhundert. Es befinden sich rund 800 Altkarten (Karten bis 1850) im Bestand. Neben topographischen Karten (Schwerpunkt Landesaufnahmen) umfasst die Sammlung auch zahlreiche thematische Karten.

2 https://phaidra.univie.ac.at/view/o:423816.

3 Die Digitalisierung erfolgt in der Abteilung Alte und wertvolle Bestände der Hauptbibliothek.

4 https://goobi-viewer.univie.ac.at/viewer/. 


\section{Dieses gesunde grüne Leuchten. Die Fachbereichsbibliothek Germanistik, Nederlandistik und Skandinavistik als Bibliotheksraum}

Die Fachbereichsbibliothek Germanistik, Nederlandistik und Skandinavistik zeichnet sich durch eine Reihe von Eigenschaften aus, die an dieser Stelle erwähnt werden könnten - sei es das umfassende Literaturangebot, die seit Jahren hohen Benutzungs- und Entlehnzahlen, die besondere Vernetzung innerhalb der Universitätsbibliothek und im Bibliothekswesen oder die Größe der primären Nutzergruppe, also die beachtliche Zahl von Studierenden und Wissenschafter_innen, die von uns mit Literatur versorgt wird. Ein Merkmal aber prägt die Fachbereichsbibliothek besonders: ihre räumliche Ausstattung und die Lage im Hauptgebäude der Universität. Sie ist Segen und Fluch zugleich: Erlaubt der markante "historische» Lesesaal besondere Angebote für die Benutzung und Veranstaltungen und die Nähe zu den betreuten Instituten und Abteilungen ebenso wie zur Hauptbibliothek eine besondere Vernetzung, zwingt sie andererseits schon immer zum Improvisieren. In sich abgeschlossen und, anders als andere Fachbereichsbibliotheken im Hauptgebäude, auch von den Institutsräumen getrennt, gilt es, für die naturgemäß wachsenden Bestände immer wieder neue Lösungen zu finden. Der Platzmangel ist evident und nicht neu, hat der damalige Bibliotheksleiter Rudolf Simek doch schon rund um den 1993 erfolgten Einzug in die Räume beklagt, die Planung wäre mehr als ein Jahrzehnt hinter dem Bedarf zurück und Raumprobleme vorprogrammiert. ${ }^{1}$ Während die damals, in den 1990er Jahren geplanten Dachbodenräume nie für die Bibliothek adaptiert wurden und heute noch leer stehen, hat sich der Buchbestand in der Zwischenzeit fast verdoppelt.

1 Rudolf Simek: Umbau historischer Räumlichkeiten für die Fachbibliothek für Germanistik an der Universität Wien. In: Österreichischer Bibliotheksbau in den Neunziger Jahren. Hg. von Otto Oberhauser. Wien: Prachner 1991 (= Biblos-Schriften 155), S. 95-102. 


\section{Raumforderungen}

Eine Situation, die nur in enger Zusammenarbeit mit den verschiedenen $\mathrm{Ab}$ teilungen innerhalb der Universitätsbibliothek und vor allem mit der nahegelegenen Hauptbibliothek zu lösen war. So wurden früh Bücher der Germanistik, die nicht mehr im Freihandbereich untergebracht werden konnten, ins sogenannte Kuppelmagazin der Hauptbibliothek ausgelagert und auf das Anwachsen des Nederlandistik-Bestands wurde mit einer Auslagerung auch der Benutzung in die Hauptbibliothek reagiert. Dieses Schicksal traf dann auch die Bücher des Kuppelmagazins, das - eine typische Episode der letzten Jahre - aufgrund anderweitigen Raumbedarfs adaptiert werden musste. Als kürzlich die Abteilung Skandinavistik, deren Raumverbund auch ein großes Kompaktmagazin umfasste, aus dem Tiefparterre des Hauptgebäudes absiedeln musste, fanden die Bibliotheksbestände eine neue Bleibe in einem Magazin im Bereich des Nordturms. Klar ist, dass damit diese nicht unbedingt glanzvolle, aber prägende Geschichte noch lange nicht zu Ende erzählt ist - nicht nur, weil die Büchermagazine im Hauptgebäude wohl in absehbarer Zeit Umbauten weichen müssen, sondern auch aufgrund der Überfüllung unserer Freihandbereiche, die immer wieder zum schmerzhaften Magazinieren ganzer oder sogar Zerreißen einzelner Signaturgruppen zwingt.

\section{Spielräume}

Doch diese kritische Zusammenfassung ist zum Glück nur ein Teil der Geschichte der letzten Jahre, auch was räumliche Aspekte betrifft. Als das Institut für Germanistik durch den Tod von Wendelin Schmidt-Dengler (1942-2008) nicht nur einen Professor für Neuere deutsche Literatur, sondern auch eine Leitfigur verlor, kam die Nachlassbibliothek als Geschenk der Familie an die Fachbereichsbibliothek und wurde, unterstützt durch Institut und Dekanat, in neuen Regalen in Seminarräumen des Instituts untergebracht - eine Lösung, die nicht nur die Funktion der Bibliothek als institutionelles Gedächtnis, sondern auch die gelungene Zusammenarbeit versinnbildlicht. In dieselbe Zeit fällt eine ähnlich erfolgreiche Lösung, als die Fachbereichsbibliothek im Zuge einer Stellenbereinigung mit der personellen Ausstattung auch den sogenannten »Bücherdienst« übernahm und damit die Aufgabe, die räumlichen Distanzen zwischen Institut für Germanistik, der Fachbereichsbibliothek und vor allem der Hauptbibliothek durch regelmäßigen Austausch zu überbrücken. Und schließlich gehört das gemeinsame Gestalten des Bibliotheksraumes vom Bestellwunsch bis zur Unterstützung bei der Vergabe der Signatur im Freihandbereich 
- etwa im komplexen Feld der systematischen Sprachwissenschaft - zur liebgewonnenen Tradition.

Im Bereich der Bibliotheksbenutzung hat sich, gerade auch für die Studierenden, eine Menge verändert. War die Fachbereichsbibliothek als Präsenzbibliothek konzipiert, die zunehmend Entlehnungen übers Wochenende - und zahllose Ausnahmen - zuließ, bietet sie nun umfassend regulär entlehnbare Bestände und die wichtige Möglichkeit, entlehnte Werke selbst vorzumerken. Zugleich steigerte das Angebot elektronischer Ressourcen - zuletzt etwa der umfassende Zugang zu E-Books des für die Germanistik wichtigen Verlags De Gruyter - die Möglichkeiten der elektronischen Nutzung und des Fernzugriffs. Auch Veränderungen der Studienpraxis, neue Studienpläne, -abläufe und -ansprüche wirken auf die Bibliotheksbenutzung. War die Bibliothek für Studierende in Diplomstudiengängen schon in frühen Übungen Arbeitswerkzeug und Thema, theoretisches Bezugs- und potenzielles berufliches Praxisfeld, ist sie für Bachelor- und Lehramtsstudien, Lehrer_innenbildung und Deutsch als Fremdbzw. Zweitsprache ein Ort der schnellen Literaturversorgung. Und während sie lange als räumliches Gegenüber der zentralen Übungsräume des Instituts fungierte, bietet sie nun, seit diese abgegeben wurden und im Tiefparterre unterrichtet wird, einen der wenigen von Studierenden, Forschenden und Lehrenden gemeinsam genutzten, frei zugänglichen und naheliegenden Raum.

\section{Der Lesesaal}

Das Zentrum all dieser Entwicklungen und Bezüge bildet der markante Lesesaal der Fachbereichsbibliothek. Der Saal, der schon vor dem Einzug der Bibliothek bestand und als »Skelettsaal« der Zoologischen Sammlung zahlreiche Präparate, manchen Totenschädel und nicht zuletzt mehrere von der Decke hängende Walskelette enthielt, hat auch heute noch einiges zu bieten: gefragte Arbeitsplätze in bester Lage und mit besonderer Atmosphäre, die jene ganz besondere Konzentration ermöglichen, die zuletzt als Privileg und Kapital wieder populär geworden ist (Stichwort: Deep Work). Wendelin Schmidt-Dengler, einer der profiliertesten Benutzer unserer Bibliothek, brachte die ambivalente Erfahrung dieses Lesesaalsettings und seine einzigartigen Folgen markant auf den Punkt: »Mystisches Dunkel herrscht, und die grünen Lampen auf den Tischen erzeugen für jeden einzelnen Leser einen Lichtkegel, der nur ihm gehört, und trennen so alle Individuen scharf voneinander. Nirgends kann man im Kollektiv so einsam sein wie in einem Lesesaal." ${ }^{2}$

2 Wendelin Schmidt-Dengler: Glanz und Lesen der Bücher. In: Sim's Das Beste 1997, S. 4-8, hier S. 8. 
Das Angebot von Leseplätzen ist natürlich nur ein kleiner Teil des umfassenden Service-Portfolios der Universitäts- und der Fachbereichsbibliothek, und die Betreuung des Lesesaals nur einer von vielen Aspekten unserer täglichen Arbeit. Und doch ist dieses "gesunde grüne Leuchten « (um es mit einer gänzlich aus dem Zusammenhang gerissenen Phrase aus den Simpsons zu sagen) ${ }^{3}$ der Lesesaallampen mehr als nostalgisches Symbol ein Zeichen fortgesetzter geisteswissenschaftlicher Arbeit und lebendiger Bibliothekspraxis.

3 The Simpsons, Staffel 8, Folge 10: The Springfield Files / Die Akte Springfield (1997). 


\section{Ein bibliothekarisches Experiment. Die Fachbereichsbibliothek Geschichtswissenschaften}

\section{Dynamik \& Zusammenführung}

Die Dynamik einer Institution lässt sich an der Frequenz der Neuerungen ablesen, die ihr Erscheinungsbild oder Image markant verändern. Im Bibliothekswesen gehört hierzu die Einführung der Suchmaschinentechnologie ebenso wie jene der bargeldlosen Gebührenabrechnung. Die Dynamik spiegelt sich aber gleichfalls in der Vielfalt jener Experimente, die nicht dem Gesetz einer allgemeinen Notwendigkeit unterliegen. Als willkommenes Experimentierfeld bot sich an der Universitätsbibliothek Wien der dezentrale Bereich mit seinen kleineren Ein- und Eigenheiten an. Keine Fachbereichsbibliothek arbeitet und funktioniert im selben Rhythmus wie die anderen, was auch für die Fachbereichsbibliothek Geschichtswissenschaften gilt. Sie befand sich um die Jahrtausendwende in einer Konsolidierungsphase. Die Fusion der beiden Institutsbibliotheken für Geschichte sowie für Wirtschafts- und Sozialgeschichte in den 1990er Jahren gehörte zu den frühesten Umstrukturierungen dieser Art und erhob noch den ambitionierten Anspruch, neben den bibliothekarischen Abläufen auch den Bestand inhaltlich zusammenzuführen. Dies inkludierte im Prozess der Online-Katalogisierung einen Dublettenabgleich ebenso wie die Auflösung thematisch parallel laufender Signaturenbereiche. Mitte der 2000er Jahre zeigten sich die Chancen, die diese aufwendige Bestandszusammenführung eröffnete, indem aufgelöste Sachgruppen mit neuen gesellschaftspolitisch relevanten Inhalten wie der Umweltgeschichte oder der Mediengeschichte gefüllt werden konnten. Die geographischen Schwerpunkte der einen Institutsbibliothek ließen sich sehr gut mit dem rein thematischen Aufbau der zweiten ergänzen. Durch die gemeinsame Anstrengung des gesamten Teams gelang es, den eingeschlagenen Weg über zwei Jahrzehnte hinweg konsequent fortzusetzen sowie 2011 letztlich auch zu einem Ende zu bringen. Die Fusion war somit ein langwieriges Experiment, das eine der größten fachhistorischen Bibliotheken Mitteleuropas, aber nicht zwangsläufig einen nachahmenswerten Modellfall hervorbrachte. Als flexibler bewährten sich in der Folge verwaltungstechnische 
Zusammenlegungen, die nach den Prinzipien einer modernen Bibliotheksgestaltung variable, räumlich oder thematisch verschieden kombinierbare Subeinheiten schufen.

\section{Bibliotheksraum \& Institute}

Die Fachbereichsbibliothek Geschichtswissenschaften teilt sich zentrale Orte ihres Selbstverständnisses und Alltags mit jenen der Institute für Geschichte und Wirtschafts- und Sozialgeschichte - vom Lesesaal über den Kopierraum bis hin zu den Sanitäranlagen. Vor der passenden Kulisse eines Blicks auf die Votivkirche bildet ein historistischer Katheder den beherrschenden Fluchtpunkt des Lesesaals, der ursprünglich ein biochemisches Unterrichtslaboratorium beherbergte. Das anachronistisch gewordene Podium eignete sich als Drehpunkt einer neuen Phase der Zusammenarbeit zwischen der Bibliothek und ihren Instituten. Es diente zunächst als überdimensionaler Ablageplatz für den wöchentlichen Bestandszuwachs und wurde dann Mitte der 2000er Jahre zum Zentrum eines multifunktionalen Begegnungsraumes. Tagsüber gehorcht der Saal den Anforderungen eines bibliothekarischen Schweigegebots, abends erhält er aber einmal im Monat seine alte Bestimmung zurück, indem hier Veranstaltungen wie Buchpräsentationen, Tagungen, Vereinssitzungen oder Diskussionsforen der Institute und ihrer Sammlungen stattfinden. Die Öffnung der Bibliothek forcierte im letzten Jahrzehnt eine neue Vereinbarung zwischen der Bibliotheksdirektion und den beiden Instituten, welche die Umgestaltung einzelner Leseplätze zu Forschungsarbeitsplätzen für Gastprofessor_innen oder Projektant_innen sowie für Besucher_innen der Sammlung Frauennachlässe und der Dokumentation lebensgeschichtlicher Aufzeichnungen enthielt. Das sensibelste Standbein einer räumlichen Kooperation zwischen Lehr- und Lesebetrieb bildet aber der Informationsschalter. Als eine der wenigen Serviceeinrichtungen der Universitätsbibliothek teilt sich die Fachbereichsbibliothek die Theke mit den Instituten, die sie als Anlaufstelle bei der Erstorientierung und der Weitervermittlung von Personen, Anfragen, Texten oder Schlüsseln nützen. Die Diensthabenden übernehmen deswegen in ihrer Verantwortung für drei Einrichtungen weitreichende Informationsaufgaben. Der gemeinsame Schalter ist ein Experiment, das ebenso wenig wie die Bestandszusammenführung Schule machen konnte. Er widerspricht dem Gedanken einer klaren Kompetenzaufteilung und eines eindeutigen Instanzenweges. Der steigende Aufwand einer Entlehnbibliothek ließ sich nur durch die enge Absprache der Bibliotheksleitung mit den Vorständen und den Sekretariaten bewältigen, die selbst mit personellen Umschichtungen durch die Schaffung von Studienservicezentren und Fachdidaktikzentren konfrontiert waren. Ungeachtet der wachsenden Hürden erwies sich 
diese Schnittstelle als ein tragfähiges strukturelles Element. Die Bedeutung eines gemeinsamen Schalters und somit auch der gegenseitigen Unterstützung von Bibliothek und Instituten spiegelte sich 2004 darin, dass eine Angestellte der Universitätsbibliothek zur historisch-kulturwissenschaftliche Fakultät mit dem Zweck wechselte, den Informationsdienst an einem neuralgischen Punkt mitzugestalten. Dieser Wechsel kann zu den Vorzeigeprojekten einer sozialen Personalpolitik an der Universität Wien gezählt werden. Mittlerweile ist die Stelle obwohl formell einer anderen Institution zugehörig - fest in die physischen wie elektronischen Bibliotheksabläufe integriert.

\section{Profil}

Das Profil der Fachbereichsbibliothek Geschichtswissenschaften, das sie von Einheiten vergleichbarer Größe unterscheidet, basiert auf speziellen Ausprägungen in der Umgestaltung von einem zweischichtigen zu einem funktional einschichtigen Bibliothekssystem, also der allmählichen Unterstellung sämtlicher Institutsbibliotheken unter eine zentrale Bibliotheksleitung. Die europäische Synchronisierung des Hochschulstudiums enthielt genügend Spielraum für verschiedenste Varianten einer Eingliederung in die Universitätsbibliotheken, was die laufenden Fortbildungsveranstaltungen für deutschsprachige Geschichtsfachreferent_innen lebendig vor Augen führen. Renommierte Universitätsbibliotheken wie jene von Freiburg im Br. verwirklichen ihre Standards als eine Teaching Library vor allem im Bereich einer zentralen Einrichtung, an der die fachlichen Ansprechpersonen der Referent_innen gemeinsame Projekte mit Lehre und Forschung ausarbeiten. Die dezentralen Standorte konzentrieren sich dagegen auf die Abwicklung bibliotheksökonomischer Abläufe. Die Leitung der Universitätsbibliothek Wien kombinierte seit den 2000er Jahren eine straffe zentrale Infrastruktur mit der Förderung einer dezentralen Informationskompetenz, die sie in die verschiedenen Phasen des fachspezifischen Curriculums von den Studieneingangsphasen bis zu den Doktorand_innenschulungen einbrachte. Dieser Weg bedingte einen pragmatischen und kompromissbereiten Aufbau der Teilbibliotheken, der sich den jeweiligen medialen Anforderungen anpasste und fließende Übergänge zwischen Modell- und Sonderfällen zuließ. In der Ausgestaltung der Fachbereichsbibliothek Geschichtswissenschaften experimentierte die Universitätsbibliothek auf zwei Ebenen, indem sie einerseits historisch unterschiedlich gewachsene Systematisierungstraditionen miteinander verschmolz und andererseits ein bibliothekarisches Kerngeschäft im Tätigkeitsbereich der Institute beließ. Beide Maßnahmen beeinflussten in erster Linie die Identifikation mit der neu geschaffenen Einrichtung. Bestand sie in der Zeit um 2000 noch immer aus zwei Buchbeständen, die eine getrennte An- 
schaffungspolitik erforderten, so wuchs sie in den letzten eineinhalb Jahrzehnten nach innen und nach außen im Bewusstsein der Lehrenden und Studierenden zu einer einzigen Bibliothek mit einem gemeinsamen Auftrag zusammen. 


\section{Von der Festung zum Aktionsraum. Die Fachbereichsbibliothek Judaistik im Wandel}

Zum Zeitpunkt der Übernahme der Leitung der Fachbereichsbibliothek Judaistik durch die Autorin im Jahre 1997 trotzte die Büchersammlung dem Institut für Judaistik an der Adresse Ferstelgasse 6, 1090 Wien, bereits jeden freien Winkel ab. Erst nach der 1998 erfolgten Übersiedelung in den neu errichteten Campus der Universität Wien konnte die Bibliothek vollständig aufgestellt werden. Die Autorin sah sich bis dahin ausschließlich als Teil des Institutsbetriebes und knüpfte am neuen Standort ihre ersten Kontakte zu erfahrenen Kolleg_innen, die sie mit einer Reihe subjektiver Sichtweisen auf den bibliothekarischen Alltag bekannt machten. Ein häufiger und geschätzter Gesprächspartner dieser frühen Jahre war ihr Erich Sommerauer, der 2008 in den Ruhestand getretene Leiter der (damals noch separat geführten) Fachbereichsbibliothek Afrikanistik, dessen fortlaufende Erzählung vom Schicksal des Alleinverantwortlichen für eine Ein-Personen-Bibliothek sie sehr beeindruckte. Er bediente sich des Bildes vom einsamen Kettenhund, der sich nie weit von seinem Platz fortbewegen durfte.

Jung und kinderlos, konnte die Autorin mit diesen Klagen damals wenig anfangen. Sie hatte ausreichend Zeit für ein ausgefülltes Privatleben und die Bibliothek war ohnehin ihr bevorzugter Aufenthaltsort. Wenn sie einmal wirklich wegmusste oder krank war, bot das Institutssekretariat einfachen Publikumsdienst an, zumindest wenn die Sekretärin vor Ort war. War sie das nicht, tat es auch eine handgeschriebene Notiz, die verkündete, dass die Bibliothek aus organisatorischen Gründen geschlossen wäre. Lückenlose Öffnungszeiten wurden damals von kleinen Zweigstellen nicht erwartet. Selbst während der eineinhalb Jahre zwischen Ende 1999 und Mitte 2001, als der Bibliothekslehrgang die Leiterin der Bibliothek weitgehend von ihrem Arbeitsplatz fernhielt, konnte der Betrieb auf diese Weise provisorisch aufrechterhalten werden. Aus diesen Gründen stellte der Zugang zu den Büchern für Benutzer_innen in gewisser Weise eine Glückssache dar. Den Gefahren des Umstands, dass dies bei vielen den Wunsch erweckte, möglichst viele Bücher zuhause zu horten, wurde 
mit der Einführung der Aleph-Entlehnung bereits im Jahr 2000 begegnet. Dadurch ging für einige Zeit alles gut.

\section{Vom Einzelkampf zur Teamarbeit}

Mit dem Antritt von Maria Seissl als Leiterin der Universitätsbibliothek Wien im Jahr 2004, bald sekundiert von Andreas Brandtner als Verantwortlichem für den dezentralen Bereich, erfolgte eine grundlegende Neubewertung des Bibliotheksbetriebes. Auf Basis der 2002 neu erlangten Teilrechtsfähigkeit der Universität Wien und befeuert durch das Potenzial des damals neuen Bibliothekssystems Aleph wurde nun der Servicegedanke zur Mission. Anstelle des Schutzes der Bücher und Bibliothekare vor potentiellen Leser_innen wurde deren $\mathrm{Zu}$ friedenheit und Wohlbefinden zum Maß aller Dinge. Der damit verbundene Wunsch nach langen, transparenten und zuverlässigen Öffnungszeiten war für eine Eine-Frau-Bibliothek wie die Fachbereichsbibliothek Judaistik nicht gut zu bewältigen und erhöhte - zusammen mit den spezifischen, stets simultan auftretenden Anforderungen an eine "working mum" und neuen Erfordernissen durch die stärkere Vernetzung der dezentralen Bibliotheken, etwa bei regelmäßigen Außenbereichssitzungen - den Druck enorm. In dieser Situation kündigte sich jedoch ein positiver Umbruch an: Stieg auf der einen Seite die Verantwortung der Bibliotheksleiterin, wurden auf der anderen Seite alte Barrieren eingerissen und ergaben sich dadurch neue Möglichkeiten. Für flankierende Maßnahmen nutzte die Bibliotheksdirektion zunächst ihre durch das Universitätsgesetz 2002 neugewonnene Freiheit im Umgang mit personellen Ressourcen, und so war es möglich, im Jahr 2005 den ersten studentischen Mitarbeiter an der Fachbereichsbibliothek Judaistik anzustellen - ein entscheidender und nachhaltiger Schritt der Befreiung der Bibliothekarin aus dem Dasein als Einzelkämpferin, der ihr schließlich eine neue, auch internationale Vernetzung und die Übernahme von neuen Tätigkeiten als Verantwortliche für den Bereich der Universitätssammlungen im Arbeitsbereich NS-Provenienzforschung ermöglichte.

Eine weitere Entlastung ergab sich durch die physische Nähe der Fachbereichsbibliotheken am Campus. Anfang 2015 wurde der durch eine neugeschaffene Durchgangstür ermöglichte gemeinsame Betrieb der Fachbereichsbibliotheken Judaistik sowie Anglistik und Amerikanistik aufgenommen. Seither werden die Benutzerdienste der Fachbereichsbibliothek Judaistik mittags oder im Bedarfsfall bereits in der Früh - an das Entlehnpult der Fachbereichsbibliothek Anglistik umgeleitet. Im Gegenzug treten die Mitarbeiterinnen der Fachbereichsbibliothek Judaistik ihrer Nachbarbibliothek sechs zusätzliche wöchentliche Dienststunden ab. Dieses Arrangement erlaubt beiden Bibliothe- 
ken, großzügige Semesteröffnungszeiten von täglich 9 bis $19 \mathrm{Uhr}$ anzubieten und beinahe das ganze Jahr verlässlichen Zutritt zu den Beständen sowie deren Ausleihe zu gewähren. In ihrer heutigen Form als einer von einem achtköpfigen Team getragenen, leistungsfähigen Bibliothek ist jene isolierte, bis über ihre Grenzen hinaus geforderte, kleine Festung, an der die Autorin vor mehr als zwei Jahrzehnten ihre Laufbahn begonnen hat, kaum mehr wiederzuerkennen. 
Open-Access-Publikation im Sinne der CC-Lizenz BY-NC-ND 4.0

(c) 2019, Vandenhoeck \& Ruprecht GmbH \& Co. KG, Göttingen ISBN Print: 9783847110989 - ISBN E-Lib: 9783737010986 


\section{Nicht nur ein Ort des stillen Studiums alter Texte. Die Fachbereichsbibliothek Klassische Philologie, Mittel- und Neulatein}

Einen weiten Weg von eher stiefmütterlich behandelten Buchbeständen des 1849 gegründeten »Philologischen Seminars« der Universität Wien hin zu einer modernen Forschungsbibliothek, die ihren Nutzer_innen nicht nur aktuelle Literatur zur Verfügung stellt, sondern sich auch als Vermittlerin von Informationskompetenz für Forschende, Lehrende und Studierende versteht, hat die heutige Fachbereichsbibliothek für Klassische Philologie, Mittel- und Neulatein hinter sich.

Ursprünglich war das 1850 zum »Philologisch-historischen Seminar» erweiterte spätere Institut für Klassische Philologie mit seiner bis in die Zwischenkriegszeit hinein eher ein Schattendasein fristenden Bibliothek bis zum Jahr 1964 dort untergebracht, wo sich später das Institut für Indogermanistik befand und wo heute Teile des Instituts für Alte Geschichte und Altertumskunde, Papyrologie und Epigraphik zu finden sind.

Lange zogen es die meisten Professoren vor, mit ihren privaten Büchern daheim zu arbeiten, so dass ein systematischer Bibliotheksaufbau nicht wirklich stattfand. Da außerdem die Benutzung der wissenschaftlichen textkritischen Ausgaben den Professoren vorbehalten war, blieb die Bibliothek für die Studierenden zunächst lange Zeit nur ein Ort des Lernens mit sehr eingeschränkten Ressourcen.

Diese Situation änderte sich erst unter der Führung von Professor Albin Lesky (1896-1981), der als erster die Notwendigkeit systematischer Ankäufe erkannte und dafür sorgte, dass ab der Zeit seiner Tätigkeit am Wiener Institut (außerordentliche Professur für Gräzistik in Wien 1932-1936, Ordinarius für klassische Philologie 1949-1967) die für die Forschung und Lehre notwendige aktuelle Literatur zielgerichtet erworben wurde.

Die Aufstellung der Bestände nach der bis heute verwendeten Systematik und die Umwandlung von einer Institutsbibliothek in eine Fachbereichsbibliothek erfolgten unter Christine Harrauer, die die Geschicke dieser Bibliothek bis zum Jahr 2007 lenkte. 
Räumlich haben sich in den letzten Jahren einige gravierende Änderungen ergeben: Im Jahr 2011 führten die wegen der neuen Brandschutzbestimmungen nötig gewordenen Bauarbeiten zum Verlust von einigen Zimmern sowie des gesamten Eingangsbereichs des Instituts und der Bibliothek. Durch die Errichtung eines eigenen Kopierraumes, in dem keine Bücher mehr aufgestellt werden durften, gingen der Bibliothek fast 60 Laufmeter an Regalen verloren. Auch die bis dahin in Professorenzimmern aufgestellten Bücher mussten im Zuge der Neubesetzung der Professuren abgesiedelt werden. So kam es sehr gelegen, dass ein Teil der Fachbereichsbibliothek Alte Geschichte mitsamt der darin befindlichen Kompaktanlage an unsere Bibliothek übergeben wurde, während die Fachbereichsbibliothek Alte Geschichte im Gegenzug die Räumlichkeiten des ehemaligen Instituts für Indogermanistik dazubekam.

An diesen neuen Standort konnten die gesamten Bestände des Mittellateins transferiert werden, wodurch für die Abteilung für klassische griechische und lateinische Literatur Platz geschaffen werden konnte, der bereits dringend benötigt wurde.

Ein dritter Standort für unsere Fachbereichsbibliothek kam schließlich im Jahr 2014 hinzu, als nach der Absiedlung des Büffets unter der Philosophenstiege die dortigen Räumlichkeiten der Abteilung für Neulatein des Instituts sowie den neulateinischen Beständen unserer Bibliothek zur Verfügung gestellt wurden.

Eine Konstante in all den Jahren ihres Bestehens war die Verankerung der Instituts- bzw. Fachbereichsbibliothek als Ort des intensiven Lernens und Studierens wie auch als Ort des nicht minder intensiven Austausches zwischen den Studierenden, die als angehende klassische Philolog_innen stets ein durchaus kommunikatives Völkchen waren.

$\mathrm{Zu}$ diesem Aspekt gesellte sich unter der Direktion von Maria Seissl im Zuge des Paradigmenwechsels, der sich durch die zunehmende Bedeutung elektronischer und somit virtueller Ressourcen abzuzeichnen begann, die hybride Wirklichkeit einer modernen Bibliothek, die ihren Nutzer_innen neben den althergebrachten analogen Medien in zunehmendem Maße auch den Zugang zu digitalen Ressourcen zur Verfügung zu stellen hat. Im Zuge dieser Entwicklung wurde rasch klar, dass die neuen Medien und die Möglichkeiten, die sie eröffneten, auch von den Nutzer_innen neue Fertigkeiten und Kenntnisse verlangten. Und wer wenn nicht die Bibliothek wäre prädestiniert dazu, diese Fertigkeiten und Kenntnisse zu vermitteln?

So ergab es sich nahezu von selbst, dass für die Vermittlung der nötigen Informationskompetenz die Zusammenarbeit zwischen der Fachbereichsbibliothek einerseits und den Mitarbeiter_innen des Instituts sowie den Studierenden andererseits noch enger wurde. Rasch etablierten sich in den Proseminaren und Seminaren, die zur Einführung in das wissenschaftliche Arbeiten dienen, fixe Lehrveranstaltungseinheiten, die ausschließlich der elektronischen 
Literaturrecherche und der Nutzung elektronischer Medien gewidmet sind. Lehrende wie Studierende erhalten auf diesem Weg einen jeweils aktuellen Einblick in das stetig wachsende Angebot an Datenbanken, E-Journals und EBooks und erfahren, wie sie diese Ressourcen am besten für ihre Arbeit nutzen können.

Auch der bereits seit langer Zeit traditionell enge Kontakt zwischen der Fachbereichsbibliothek und den AHS-Lehrer_innen für Latein und Griechisch hat sich in den letzten Jahren noch verstärkt, da in zunehmendem Maße bereits Schüler_innen im Zuge der Erstellung ihrer Vorwissenschaftlichen Arbeiten die Angebote der Universitätsbibliothek und ihrer Fachbereichsbibliotheken nützen. Das beginnt bei Bibliotheksführungen für Schulklassen und reicht bis zu gezielten praktischen Hilfestellungen bei der konkreten Literaturrecherche und zum korrekten Zitieren.

Ebenfalls eine Folge dieser engen Kooperation mit Schulen sind die nicht wenigen Schüler_innen, die aufgrund ihres Interesses an den Fächern Latein bzw. Griechisch oder bereits im Hinblick auf ein angestrebtes Studium der Klassischen Philologie den Wunsch haben, in ihren Ferien ein Praktikum oder Volontariat in der Fachbereichsbibliothek zu absolvieren. In jedem Fall ist es immer wieder schön zu sehen, mit wie viel Freude und Engagement sich die künftigen Forscher_innen und Lehrer_innen in den Bibliotheksbetrieb einbringen.

Neben der Zusammenarbeit mit Schulen hat sich auch eine zunehmende Vernetzung mit anderen Institutionen wie dem Archiv der Österreichischen Akademie der Wissenschaften sowie den Fachbibliotheken für Klassische Philologie anderer Universitäten entwickelt. Auf diese Weise konnte zum Beispiel der sowohl von seinem Umfang als auch von seinem Inhalt her bedeutende Nachlass des emeritierten Ordinarius Hans Schwabl (1924-2016) im Jahre 2016 zum Großteil an die Fachbereichsbibliothek des Instituts für Klassische Philologie an der Comenius-Universität Bratislava weitergeleitet werden, wo er dabei hilft, Lücken im dortigen Bestand zu ergänzen und den slowakischen Kolleg_innen zahlreiche Standardwerke zur Erforschung des Lateinischen und Griechischen wie auch der Religionsgeschichte zur Verfügung zu stellen. Dem Engagement von Jana Grusková von der Comenius-Universität Bratislava ist es zu verdanken, dass dieser Büchernachlass sogar geschlossen aufgestellt und so als Bibliothekscorpus erhalten werden konnte. Der ebenfalls in diesem Nachlass enthaltene Bestand an Sonderdrucken und Archivalien (insbesondere Briefen), der vor allem im Hinblick auf Hans Schwabls Tätigkeit und internationale Vernetzung als wirkliches Mitglied der Österreichischen Akademie der Wissenschaften von Bedeutung ist, konnte dem dortigen Archiv übergeben werden, wo er als geschlossener Bestand erhalten und für die Forschung zugänglich gemacht wird. 
Ebenfalls dem guten Kontakt zu unserer Schwesterbibliothek in Bratislava ist es zu verdanken, dass Mária Májeková, eine junge Kollegin aus dem dortigen Institut, ein Semester lang als Erasmus ${ }^{+}$-Traineeship-Praktikantin in unserer Fachbereichsbibliothek tätig sein konnte. Mittels desselben Austauschprogramms durften wir auch Julija Hoda vom Institut für Klassische Philologie der Universität Ljubljana für ein Semester als Praktikantin bei uns haben. Beide Kolleginnen haben unser Team mit großem Engagement verstärkt und damit den Grundstein für immer noch bestehende gute Kontakte und weitere Kooperationen gelegt.

In den 170 Jahren ihres Bestehens hat die Fachbereichsbibliothek Klassische Philologie, Mittel- und Neulatein also bereits einen langen und wechselvollen Weg hinter sich gebracht, der dank der aktuellen Entwicklungen auf dem Bibliotheks- und Informationssektor auch für die Zukunft neue und spannende Herausforderungen verspricht, die Garant dafür sind, dass hier weiterhin mehr stattfindet als nur das stille Studium alter Texte. 


\section{Sammeln ist unsere Leidenschaft (Daten, Objekte, Menschen). Die neuen Herausforderungen der Fachbereichsbibliothek Kultur- und Sozialanthropologie}

Die Fachbereichsbibliothek Kultur- und Sozialanthropologie hat sich in den letzten Jahren von einer kleinen Institutsbibliothek zu einer Drehscheibe vieler verschiedener Arbeitsprozesse entwickelt. Neben den vielfältigen Aufgaben einer Fachbereichsbibliothek werden laufend neue interessante Herausforderungen an uns herangetragen. Wo eine Kooperation sinnvoll erscheint und ressourcentechnisch zu bewältigen ist, übernehmen wir gern eine gestalterische, kooperative oder unterstützende Funktion.

So wurde das Projekt »Ethnographische Datenarchivierung (EDA)« 2017 an die Fachbereichsbibliothek Kultur- und Sozialanthropologie angedockt und damit auch die Sammlung des Instituts für Kultur- und Sozialanthropologie näher an die Bibliotheksagenda herangerückt. Der Sammlungsbestand umfasst ca. 1.000 ethnographische Objekte, ca. 30.000 Fotos und Dias, 1.200 Filme, ca. 80 großformatige Bildtafeln, 15 Gemälde und eine Großmaske. Kooperationen mit dem Institut für Kultur- und Sozialanthropologie entstehen bei gemeinsamen Veranstaltungen und Ausstellungen, und es ist erfreulich, dass die Mitglieder des Instituts, aber auch die Studierenden, an unseren Bestrebungen teilhaben.

Ein herzliches Dankeschön gilt all den Mitarbeiter_innen der Fachbereichsbibliothek (seit 2010 waren es insgesamt 26), die seit nunmehr neun Jahren alle Aufgaben mittragen und durch ihr Engagement unsere Arbeit zum Erfolg führen.

\section{Das Projekt »Ethnographische Datenarchivierung (EDA)«}

Das Pilotprojekt "Ethnographische Datenarchivierung" ist eine Kooperation zwischen dem Institut für Kultur- und Sozialanthropologie und der Universitätsbibliothek Wien, angesiedelt an der Fachbereichsbibliothek Kultur- und Sozialanthropologie. Das Projekt wurde im Februar 2017 für die Dauer von zwei Jahren mit zwanzig Wochenstunden eingerichtet, besetzt durch Igor Eberhard, und konnte nun bis Ende 2020 verlängert werden. Ziel des Projekts ist es, vor- 
handenes und zukünftiges Feldforschungsmaterial zu digitalisieren und einer Langzeitarchivierung zuzuführen. Die dafür notwendigen Strukturen sollen aufgebaut, Workflows entwickelt und die notwendigen Geräte (z. B. Diascanner) angeschafft werden.

Die Projektleitung liegt organisatorisch bei der Leitung der Dienstleistungseinrichtung Bibliotheks- und Archivwesen und wissenschaftlich beim Institut. Das konkrete Projektmanagement wird von der Fachbereichsbibliothek Kulturund Sozialanthropologie getragen. Studentische Mitarbeiter_innen, Praktikant_innen und Lehrlinge der Universitätsbibliothek unterstützen das Projekt zeitweise.

Die Ausgangssituation des Projekts umfasst das Datenmaterial des ethnographischen Archivs (zurückreichend bis 1930) mit ca. 30.000 Fotos, über Jahrzehnte gesammelte Datenbestände der Ethnolog_innen des Instituts für Kultur- und Sozialanthropologie aus Feldforschungsaufenthalten sowie laufend neue ethnographische Daten aus aktueller Forschung. Das vorliegende Material besteht vor allem aus audiovisuellen Aufnahmen, Tonaufnahmen, Feldforschungsnotizen und -tagebüchern sowie Dias und Fotos.

$\mathrm{Da}$ in naher Zukunft viele der Institutsmitglieder in Pension gehen werden, wollen wir den nachhaltigen Zugang zu ihren Forschungsdaten gewährleisten und die Metadaten international sichtbar und zugänglich machen.

Heute ist ein umfassendes Forschungsdatenmanagement auch im Fach Kultur- und Sozialanthropologie anzustreben. Es besteht die Notwendigkeit, die Best-Practice-Methoden für Feldforschung und Forschungen überhaupt herauszufinden, da die Ansprüche der Fördergeldgeber immer höher werden und in vielen Fällen ein Nachweis der Langzeitspeicherung der Daten in Forschungsprojekten erbracht werden muss. Im Rahmen des Projekts wird die Entwicklung eines Forschungsdatenmanagements angestrebt.

In der abteilungsübergreifenden Zusammenarbeit mit den für PHAIDRAzuständigen Mitarbeiter_innen an der Universitätsbibliothek und am Zentralen Informatikdienst konnten diese Voraussetzungen berücksichtigt werden. Technische Abläufe wurden nach deren Klärung umgesetzt und Lösungsstrategien erarbeitet. So konnte auf die speziellen Anforderungen der ethnologischen Forschung eingegangen und die Eingabemaske in PHAIDRA modifiziert werden. Die erste Testung war Gegenstand eines Projekts des Universitätslehrgangs Library and Information Studies 2018. Mittlerweile wurde die neue Eingabemaske fertiggestellt und wird derzeit auf ihre Funktion und Anwenderfreundlichkeit überprüft. Erstmals können alle relevanten Metadaten von zusammengehörigen Objekten (z. B. Foto auf Karton mit Beschreibung auf der Rückseite) in der neu entwickelten Funktion "Containerobjekt" erfasst und die Digitalisate hochgeladen werden. Durch die Eingabemöglichkeit der Kontextdaten steht nun 
eine Submit-Form zur Verfügung, die weitestgehend alle Anforderungen ethnographischer Forschung für die Langzeitarchivierung erfüllt.

\section{EDA - Nationale und internationale Kooperationen}

Durch die Teilnahme an Tagungen und Workshops in Österreich und Deutschland werden alle heiklen Fragen (Zugriffsrechte, Datenschutz, ethische Fragen etc.) rund um die Datenarchivierung diskutiert und gemeinsam an Lösungsansätzen gearbeitet. Das Schöne ist, dass alle fachverwandten Institutionen mit Digitalisierungsbestrebungen zurzeit an denselben Fragen arbeiten, daher bieten Kooperationen wertvolle Hilfestellungen und das Potential für Weiterentwicklung. Mittlerweile hat sich das EDA-Projekt als wichtiger Player mit Vorreiterfunktion etablieren können.

Kooperationen mit ethnologischen Digitalisierungsinitiativen bestehen mit einer Reihe an Institutionen: Humboldt-Universität Berlin, Freie Universität Berlin, EVIFA - virtuelle Fachbibliothek Ethnologie (Berlin), Koordinierungsstelle für wissenschaftliche Universitätssammlungen in Deutschland, Universität Hamburg und vielen mehr; in Österreich mit der Österreichischen Akademie der Wissenschaften, dem Phonogrammarchiv, dem Weltmuseum und der von unserem Projektmitarbeiter gegründeten AG Daten. Es werden laufend mehr. Gerne nutzen wir die Gelegenheit, auf Tagungen, Workshops oder bei anderen Veranstaltungen zum Thema unsere Arbeit im Projekt vorzustellen und unsere Ergebnisse zu präsentieren bzw. zu eigenen Veranstaltungen einzuladen.

\section{Ethnographische Sammlung des Instituts für Kultur- und Sozialanthropologie}

Durch die ausgezeichnete Zusammenarbeit mit der Sammlungskoordinierungsstelle der Universität Wien ${ }^{1}$ gelang es, die Sammlung von ihrem Dämmerschlaf (nur unterbrochen durch zweimaligen Schimmelbefall) zu befreien, und eine funktionierende Schausammlung aufzubauen. Der Weg dorthin war herausfordernd und es ist auch noch nicht alles durchgestanden, die Anforderungen sind vielfältig und die Ressourcen gering, doch zuversichtlich arbeiten wir weiter.

Weshalb ist diese Arbeit erforderlich? Im Rahmen des Projekts EDA musste auch die Sammlung mitgedacht werden, denn Institutsmitglieder möchten nicht

1 Siehe den Beitrag von Claudia Feigl in diesem Band. 
nur ihre Datenbestände für die Nachwelt erhalten wissen, sondern auch einen Platz für ihre ethnographischen Objekte finden, die sie über die Jahrzehnte von ihren Forschungspartnern geschenkt bekommen oder selbst erworben haben. Die Abgabe in die Sammlung ist aber nur dann denkbar, wenn diese in gutem Zustand ist und die Objekte ordnungsgemäß behandelt werden. Außerdem kann eine Sammlung nur dann erfolgreich in Lehre und Forschung eingesetzt werden, wenn die Betreuung nachhaltig gewährleistet ist.

2017 begann die Arbeit im Keller des Neuen Institutsgebäudes. Nach Begutachtung durch eine Expertin für Schimmel und einem Experten für Insektenbefall wurden wir in der Reinigung der Objekte unterwiesen und führten diese mit Hilfe eines gemieteten Museumsstaubsaugers in Vitrinen und auf Regalböden durch. Die Objekte durften so wenig wie möglich bewegt werden. Glücklicherweise gab es keinen Schimmel und der Insektenbefall hielt sich in Grenzen.

Danach widmeten wir uns der Inventur der ethnographischen Objekte - eine mühsame, aber sehr interessante Arbeit, bei der über tausend Gegenstände, vorwiegend aus Afrika, Asien und Ozeanien, verzeichnet wurden. Mit vielen Dingen musste äußerst sorgsam umgegangen werden, da sie teilweise in sehr schlechtem Zustand sind. Besonderer Respekt galt Objekten aus menschlichen Überresten. Viele Ethnien verwenden solche Objekte als Kultwerkzeuge für religiöse Handlungen, Glaubensträger mit mystischen Verbindungen zur Götterwelt, manche dürfen nur vom Schamanen oder vom Stammesoberhaupt berührt werden. Als später eine Übersiedlung der Sammlung vorgenommen werden musste, wurden für den neuen Depotraum neue Regalsysteme angeschafft und eine Aufstellungsart gewählt, die den direkten Kontakt mit den Objekten zulässt. Ein fix eingerichtetes Monitoring gewährleistet ein konstantes Raumklima.

Als Schausammlung konzipiert, soll sie Anschauungsobjekte für die Studierenden bereithalten. Durch die umfassende Unterstützung der Sammlungsbeauftragten konnten wichtige Maßnahmen zur Bestandserhaltung getroffen werden. Die Auseinandersetzung mit den Objekten, die Recherche über ihre Herkunft und die Umstände ihres Erwerbs werden jedoch noch viel Zeit in Anspruch nehmen. Laufend werden Studierende in Form von Lehrveranstaltungen, aber auch studentische Mitarbeiter_innen und Praktikant_innen der Fachbereichsbibliothek zur Mitarbeit in der Sammlung und zur wissenschaftlichen Bearbeitung der Objekte eingesetzt. 


\section{Fachbereichsbibliothek Kultur- und Sozialanthropologie - eine Bibliothek mit sozialer Verantwortung}

Die Fachbereichsbibliothek Kultur- und Sozialanthropologie versteht sich nicht nur als Serviceeinrichtung, sondern möchte interessierten Personen die Chance geben, durch eine geringfügige Anstellung, im Rahmen eines Praktikums oder als Lehrling in der Arbeitswelt Bibliothek Fuß zu fassen. Oft genügt nur eine kleine Chance, um sich weiter orientieren zu können und neue Wege zu beschreiten. Einige ehemalige Mitarbeiter_innen der Fachbereichsbibliothek sind inzwischen national wie international in der Wissenschaft, in der Kunst oder in der Politik tätig.

Die Fachbereichsbibliothek Kultur- und Sozialanthropologie erwies sich zudem in den letzten Jahren mehrfach als Dienstort für Kolleg_innen, die hausintern ein neues Aufgabengebiet suchten. Angesichts der hohen Fluktuation der studentischen Mitarbeiter_innen - nach Abschluss des Studiums zieht es die meisten in die weite Welt hinaus - wird an der Fachbereichsbibliothek ein besonderes Augenmerk auf das »Teambuilding " gelegt. Entsprechende Aktivitäten helfen, einander besser kennen und verstehen zu lernen.

Das Team der Fachbereichsbibliothek ist neben den klassischen Bibliotheksaufgaben auch immer wieder engagiert in Ausstellungsprojekten eingebunden. Bereits drei Mal wurden kleine Ausstellungen zu Spezialthemen in den Gang-Vitrinen im vierten Stock des Neuen Institutsgebäudes kuratiert und interessante Bücher, Fotos und Karten aus unseren Bibliotheksbeständen gezeigt Bisher waren dies im Gedenkjahr 2018: Verfolgung - Vertreibung - Ermordung. Schicksale von Wiener Völkerkundler_innen in der NS-Zeit; 2017: Wohn.Welten. Indigene Architektur trifft auf Moderne; und 2016 die Buchausstellung Kon/ Front. Gegenüberstellung alter und neuer Darstellungen zu Körperkunst (Kooperation mit dem Universitätslehrgang Library and Information Studies).

$\mathrm{Zu}$ den vielfältigen Aktivitäten zählen auch Buchflohmärkte im Rahmen von KSA-Konferenzen, die Mitgestaltung bei Tagen der offenen Tür und am Töchtertag sowie die Kooperation bei Veranstaltungen des Instituts für Kultur- und Sozialanthropologie und der universitären Sammlungen. All dies verlangt hohe Bereitschaft zu Engagement und Flexibilität im Team der Fachbereichsbibliothek. Der große Handlungsspielraum, welcher durch den offenen Führungsstil der Leiterin der Universitätsbibliothek ermöglicht wird, erlaubt Engagement, Kreativität und Eigenverantwortung als Voraussetzung dafür, dass Neues entstehen kann. 
Open-Access-Publikation im Sinne der CC-Lizenz BY-NC-ND 4.0

(c) 2019, Vandenhoeck \& Ruprecht GmbH \& Co. KG, Göttingen ISBN Print: 9783847110989 - ISBN E-Lib: 9783737010986 


\section{Martin Steinreiber}

\section{Mit 167 Jahren in die Zukunft. Die Fachbereichsbibliothek Kunstgeschichte}

Die Fachbereichsbibliothek Kunstgeschichte der Universitätsbibliothek Wien nimmt eine besondere Stellung ein. So gehört sie nicht nur im Verbund der Universitätsbibliothek Wien zu den älteren Bibliotheken, sondern ist insgesamt eine der ältesten universitären Kunstbibliotheken im deutschen Sprachraum, wurde sie doch 1852 gemeinsam mit dem Lehrstuhl für Kunstgeschichte an der Universität Wien eingerichtet.

Der Bestand spiegelt dem entsprechend die Forschungsschwerpunkte wider, die im Laufe des fast 170-jährigen Bestehens des Instituts für Kunstgeschichte gesetzt wurden. So sammelte man bereits sehr früh neben Büchern zur europäischen Kunst Publikationen zur byzantinischen, asiatischen und zur islamischen Kunstgeschichte. Diese intensive Auseinandersetzung mit außereuropäischer Kunstgeschichte am Institut mündete zuletzt 2012 in die Neuschaffung einer eigenen Professur für islamische und 2016 in eine Professur für chinesische Kunstgeschichte. Und das Institut wächst aufgrund einer Vielzahl von mit den Professuren verbundenen Forschungsprojekten weiter. Alle diese neuen Fachbereiche wollen mit aktueller Literatur versorgt werden.

Wachstum ist eines der prägendsten Kennzeichen der gesamten Universität Wien, die heute die größte Universität des deutschsprachigen Raums und eine der größten Universitäten Europas ist. Im Rahmen der Leistungsvereinbarung 2019 bis 2021 mit der Republik Österreich konnte die Universität zusätzlich 120 Millionen Euro lukrieren, die in die Besetzung 73 neuer Professuren investiert wird. Damit plant die Universität, nicht nur die Studienbedingungen weiter zu verbessern, sondern will auch zukunftsorientierte Akzente in der Forschung setzen. ${ }^{1}$

Die Universitätsbibliothek Wien unterstützt diese gesamtuniversitäre Entwicklung und hält mit ihr Schritt. Als Teilbibliothek der Universitätsbibliothek Wien kann die Fachbereichsbibliothek Kunstgeschichte mit ihrer breiten

1 Vgl. https://rektorat.univie.ac.at/strategie/leistungsvereinbarung/ [Letzter Zugriff: 05.01. 2019]. 
Sammlungstätigkeit als pars pro toto gesehen werden. So muss die benötigte, zum größten Teil nur physisch existierende Literatur nicht nur aus China, dem Iran oder Russland beschafft werden, die Werke müssen auch die Titelaufnahme in korrekten Transliterationen durchlaufen, und eine entsprechende Sichtbarmachung des Inhaltes durch gute Beschlagwortung ist notwendig. Die Universitätsbibliothek Wien hat klar erkannt, dass hierfür Knowhow aufgebaut, neue Ressourcen geschaffen und neue Wege zur Informationsbeschaffung beschritten werden müssen.

Derzeit versorgt die Fachbereichsbibliothek Kunstgeschichte das Institut für Kunstgeschichte mit seinen rund 100 wissenschaftlichen Mitarbeiter_innen sowie 1.600 aktiv Studierenden mit fachrelevanter Literatur. An das Institut ist das Pächt-Archiv, ein Forschungszentrum für Buchmalerei des Mittelalters und der frühen Neuzeit angegliedert; auch die hier arbeitenden Wissenschafter_innen nutzen die Fachbereichsbibliothek. Es liegt wohl in der Natur des Faches Kunstgeschichte, dass es zudem eine große Zahl an außeruniversitären Nutzer_innen gibt, die die Bestände der Fachbereichsbibliothek einsehen. Dazu zählen nicht nur interessierte Laien, sondern auch Angehörige anderer Forschungseinrichtungen, aber vor allem Mitarbeiter_innen unterschiedlichster Museen, und hier wiederum Ausstellungskurator_innen, die den in der Freihandaufstellung bedingten, unkomplizierten Zugriff auf den wertvollen Literaturbestand sehr schätzen.

Der physische Bestand wächst im Durchschnitt um 2.500 Bände pro Jahr. So gilt es, eine Vielzahl an nationalen und internationalen Ausstellungskatalogen zu erwerben. Aktuelle Publikationen aus dem mittel- und westeuropäischen sowie aus dem gesamten englischsprachigen Raum werden angekauft, nicht zu vergessen sind die Werke zur außereuropäischen Kunst. Zudem müssen alle Epochen abgedeckt werden, beginnend mit ausgewählten Büchern zur antiken Kunst, über Publikationen zu Mittelalter, Renaissance und Barock, bis hin zu den Kunstströmungen des 19., 20. und 21. Jahrhunderts, denn auch Gegenwartskunst spielt eine gewichtige Rolle in Lehre und Forschung. Selbstverständlich wird so breit wie möglich Literatur zur Kunst Österreichs in seinen derzeitigen, aber auch historischen Grenzen erworben.

Wie erfolgt nun der Bestandsaufbau? Neben dem klassischen Ankauf ist die Fachbereichsbibliothek Kunstgeschichte in der glücklichen Lage, immer wieder großzügige Schenkungen (z. B. Liechtenstein Museum, TBA21, Kunsthalle Wien) und Nachlässe (z.B. jener der bekannten Orientalistin Dorothea Duda) zu erhalten. Aber auch der Tausch spielt nach wie vor eine wichtige Rolle. Die Fachbereichsbibliothek Kunstgeschichte ist seit 2017 Mitglied der Elektronischen Tauschbörse Kaiserslautern (ELTAB) und konnte so in ein europaweites, bis nach St. Petersburg zur Russischen Nationalbibliothek reichendes Netzwerk einstei- 
gen, um auf unkomplizierte Art Publikationen einzutauschen, die ansonsten nur sehr kostenintensiv zu beschaffen wären.

Mit Stand Jänner 2019 verfügt die Fachbereichsbibliothek Kunstgeschichte über einen physischen Bestand von rund 140.000 Bänden, beginnend mit dem 1512 in Paris erschienenen Büchlein Omnium Angeli Politiani operum, bis hin zur aktuellsten Literatur. Vom Format her kann von 8,6 x 6,2 cm bis $97 \times 71 \mathrm{~cm}$ jede Buchgröße geboten werden, das wertvollste Werk ist wohl Fischer von Erlachs Entwurffeiner historischen Architektur in der Wiener Ausgabe von 1721.

Ergänzt wird der Bestand durch 250 Zeitschriftentitel und Mikroformen. Dazu kommen die elektronischen Ressourcen (Bilddatenbanken, E-Books usw.), die einen immer größer werdenden Teil des Gesamtbestandes der Universitätsbibliothek ausmachen.

Die Fachbereichsbibliothek Kunstgeschichte gilt aber nicht nur als Ort der Recherche, sondern wird verstärkt auch als Ort des Lernens und des gemeinsamen Arbeitens gesehen. Dies wird auch von den Zutrittszahlen untermauert, die sich gerade in den letzten Jahren massiv erhöht haben (2018: rund 80.000). Die Kombination aus 200 Arbeitsplätzen und den langen Öffnungszeiten (im Semester: Mo.-Fr. jeweils 9-18 Uhr, Sa. 10-18 Uhr) machen die Fachbereichsbibliothek zu einem gut genutzten studentischen Working Space. Im letzten Jahr wurde, um den vor allem ab Semestermitte aufkommenden Ansturm etwas abzufedern, ein eigener Gruppenarbeitsraum mit zwölf zusätzlichen Arbeitsplätzen eingerichtet.

Neben der Bewältigung der bereits klar umrissenen Anforderungen gilt es aber auch, die Fachbereichsbibliothek Kunstgeschichte für die Zukunft fit zu machen. Kunsthistoriker_innen sind es gewohnt, in langfristigen Zeiträumen zu denken. Das gilt auch für Bücher, die nicht nur als über Jahrhunderte reichende Träger relevanter Information gesehen werden, sondern auch als Zeitdokumente und Zeugen der Wissenschaftsgeschichte, die z.B. anhand ihrer Ex Libris und Marginalien Auskunft geben. Daher ist neben dem Bestandsaufbau auch die langfristige Bestandserhaltung ein wesentliches Betätigungsfeld der Fachbereichsbibliothek.

Zusätzlich zu diesen traditionellen Aufgaben muss sich die Fachbereichsbibliothek Kunstgeschichte aber auch auf die neuen Erfordernisse einstellen, um weiterhin als lebendige Kunstbibliothek wahrgenommen zu werden. So gilt es, den relativ jungen Zweig der Digitalen Kunstgeschichte in Zusammenarbeit mit den an der Universitätsbibliothek Wien bereits etablierten Fachabteilungen (z.B. E-Resource Management, Open Access Office, Repositorienmanagement PHAIDRA) verstärkt zu unterstützen, sei es in Form von Digitalisierung urheberrechtsfreier Kunstliteratur und Quellendokumenten, Zurverfügungstellung von Datenbanken und Bearbeitungstools, oder mit der Unterstützung visueller 
Forschungsmethoden. Die Fachbereichsbibliothek Kunstgeschichte nimmt in diesem Zusammenhang verstärkt ihre Rolle als Informationsvermittlerin wahr.

Dem entsprechend muss auch die elektronische Ausstattung sowohl physisch als auch digital weiterentwickelt werden (z. B. neuere, hochauflösende Scanner, schnellere PCs, unkomplizierte Druckmöglichkeiten, Pflege der Datenbanken, Weiterentwicklung des E-Book-Angebots).

Allein dieser kurze Ausblick bestätigt den Wandel, welchem die Bibliothekswelt unterworfen ist. Unter der Leitung von Maria Seissl nimmt die Universitätsbibliothek Wien diesen Wandel an und gestaltet ihn aktiv mit. Als nachhaltigstes Beispiel sei der 2017 erfolgte Systemwechsel von Aleph auf Alma genannt, der in Österreich ohne die Federführung der Universitätsbibliothek Wien wohl nicht in dieser Form möglich gewesen wäre.

Die Universitätsbibliothek Wien im Großen und mit ihr die Fachbereichsbibliothek Kunstgeschichte im Kleinen rüsten sich somit für die kommenden Veränderungen und sorgen dafür, dass die Fachbereichsbibliothek Kunstgeschichte auch in den nächsten 167 Jahren eine gesuchte Anlaufstelle für Kunsthistoriker_innen bleiben wird. 


\section{Small is beautiful. Die Fachbereichsbibliothek Musikwissenschaft}

Die Fachbereichsbibliothek Musikwissenschaft ist eine jener früheren Institutsbibliotheken der Universität Wien, die in den letzten Jahren in die Universitätsbibliothek eingegliedert wurden. Bestimmte Merkmale funktionaler Einschichtigkeit, insbesondere den Geschäftsgang betreffend, waren schon vor der Umwandlung erreicht worden, sodass sich die Übergabe an die Universitätsbibliothek 2008 auf das Übertragen der Personal-, Raum- und Bestandsverantwortung beschränkt hat. Nichtsdestoweniger wurde dieser letzte Schritt lokal als der gravierendste empfunden: nicht nur, weil etwas, das von Beginn an mit dem Institut verbunden und über Jahrzehnte eigenverantwortlich aufgebaut worden war, in die Hände Dritter gegeben werden sollte, sondern damit auch die Hoheit über das vorrangige eigene Arbeitsinstrument.

Diese Umwandlung ist nun etwas mehr als zehn Jahre her. Vor dem Hintergrund der damaligen Befürchtungen seien hier Überlegungen angestellt, wie sich die Bibliothek aus Sicht ihrer Benutzer_innen entwickelt hat.

\section{Nutzer_innen als Hindernis?}

Die Umwandlung der Bibliothek des Instituts für Musikwissenschaft in eine Fachbereichsbibliothek spielte sich vor dem Hintergrund eines jahrzehntelangen, gut begründeten Trends ab. Wesentlicher Motor der Weiterentwicklung von Bibliotheken in Richtung (funktionaler) Einschichtigkeit sind übergeordnete strategisch-wirtschaftliche Argumente, um die es hier jedoch nicht gehen soll: Auf der Ebene einer Teilbibliothek liegen die unmittelbaren Gestaltungsmöglichkeiten mehrheitlich in operativen Agenden. Auch dort sind die Schattenseiten einer Bibliotheksverwaltung unter der Hoheit wissenschaftlichen Institutspersonals jedoch augenfällig: wenig Ausgleich zwischen den Institutsinteressen und jenen anderer Nutzer_innengruppen; formaliter oft Präsenzbibliothek, realiter ebenso oft an Laissez-faire grenzende Entlehnbedingungen für das Personal; permanenter Bibliothekszugang für das Personal mit Auswirkungen auf die Verbuchungsmoral 
außerhalb der Öffnungszeiten; kostspielige Parallelankäufe zu anderen Institutsbibliotheken; eingeschränkte Nachhaltigkeit in der Qualität der Bibliotheksverwaltung an sich, weil sie Nebengeschäft ist statt Kernaufgabe etc. Angesichts dieser offenkundig privilegierten Stellung des wissenschaftlichen Personals wurden seine Einwände im Zuge dieser an zahlreichen Universitäten angestoßenen Veränderungsprozesse oft als Besitzstandswahrung eingeordnet, als zu überkommendes Hindernis für einen effizienten Bibliotheksbetrieb. ${ }^{1}$

\section{Perspektivenwechsel}

Aber entsteht diese gewiss naheliegende Sicht auf die Dinge nicht zu einseitig vom Standort der Verwaltung aus? Haben die vielerorts hartnäckigen und andauernden Einwände vor allem geisteswissenschaftlichen Personals ${ }^{2}$ gegen diesen Strukturwandel - systematisch gedacht - nicht Berechtigung? Die methodisch, zeitlich und lokal unabhängig voneinander angestellten Überlegungen zum geisteswissenschaftlichen Forschungsprozess von Bernhard Fabian ${ }^{3}$ und Andrew Abbott ${ }^{4}$ ähneln einander in der Konsequenz sehr: Die systematisch vom Zufallsfund und der intraindivuellen Informationsverarbeitung abhängige Natur bibliotheksrecherchelastiger Forschung ist von einer möglichst umfassenden, unmittelbar verfügbaren, multipel geordneten, universalen Bibliothek abhängig: "Sie muß dem Forscher erlauben, seinen spontanen Einfall mit größtmöglicher Effizienz am empirischen Material zu überprüfen. Sie muß ihn zudem in die Lage versetzen, eine Fragestellung durch die Primär- und Sekundärliteratur verfolgen zu können - gleichviel wohin der Weg führt. . ${ }^{5}$ Fabian spricht von einer »universalen Präsenzbibliothek « als der »idealen Norm«.6

Die Bedingungen, die in einer typischen Institutsbibliothek bestehen, bringen sie dieser Norm sehr nahe: Literaturauswahl durch die Forscher_innen entlang der konkreten Forschungsinteressen, unmittelbare Nähe zum Bestand,

1 Vgl. etwa Axel Halle: Strukturwandel der Universitätsbibliotheken. Von der Zweischichtigkeit zur funktionalen Einschichtigkeit. In: Zeitschrift für Bibliothekswesen und Bibliographie 49 (2002), H. 5-6, S. 268-270.

2 Für Beispiele siehe etwa Tobias Buck: Kritische Erfolgsfaktoren funktionaler Einschichtigkeit unter besonderer Berücksichtigung des Bibliothekssystems Universität Hamburg, Institut für Bibliotheks- und Informationswissenschaft der Humboldt-Universität zu Berlin. Berlin 2013 (Berliner Handreichungen zur Bibliotheks- und Informationswissenschaft, Heft 346), S. $32 \mathrm{f}$.

3 Bernhard Fabian: Buch, Bibliothek und geisteswissenschaftliche Forschung. Zu Problemen der Literaturversorgung und der Literaturproduktion in der Bundesrepublik Deutschland. Göttingen: Vandenhoeck und Ruprecht 1983, S. 32-36.

4 Andrew Abbott: The Traditional Future: A Computational Theory of Library Research. In: College \& Research Libraries 69 (2008), H. 6, S. 524-545.

5 Fabian, S. 34.

6 Fabian, S. 36. 
Dubletten $\mathrm{zu}$ anderen Bibliotheken, wo aus Gründen einer »spezifischen Universalität« lokal notwendig, permanent möglicher Zugang, wenige Entlehnungen außer Haus, hoher Präsenzanteil der Bestände. Elektronische Ressourcen ergänzen heute die gedruckten Materialien und erhöhen den Komfort, aber sie ersetzen die Unmittelbarkeit des Zugangs zur physischen Bibliothek vor allem für Primärquellen und die monographische Literatur des 20. Jahrhunderts nicht, »because bibliographical chains will bounce in and out of the print materials, and you can't afford to wait till you get to the library to follow a chain «"

\section{Von der Institutsbibliothek zur Fachbereichsbibliothek}

Angesichts der Diskrepanz zwischen übergeordneten Effizienzerfordernissen und den Spezifika typischer Anwendungsprozesse war es Zielsetzung der Fachbereichsbibliothek Musikwissenschaft, auf letztere auch nach der Umwandlung so bewusst Rücksicht zu nehmen wie möglich. So ist es zum Beispiel eine bewusste Befürwortung und kein Einknicken, bis heute Bibliotheksschlüssel an das etwa 35köpfige Kollegium auszugeben. Selbstverständlich führt das im Einzelfall zu Fehlverhalten, das korrigiert werden muss (und kann), aber der Zugang rund um die Uhr ist objektiv gesehen von solch großem Nutzen, dass die an einer Hand abzählbaren jährlichen administrativen Zwischenfälle in Kauf genommen werden können. Da aus Platzgründen kein eigener Forschungslesesaal ermöglicht werden kann, sind nicht entlehnbare Bestände - das betrifft vor allem Editionen von Primärquellen - für das Personal entlehnbar. Literaturwünsche werden unmittelbar erfüllt (bei Dublettenbildung nach Rücksprache), sodass die Literaturauswahl immer den Bedarfen der Wissenschafter_innen entspricht, auch wenn damit das Bibliotheksbudget so ausgeschöpft wird, dass eine fachbibliothekarisch-systematische Erwerbung ausgeschlossen ist. Damit haben sich die drei grundlegenden Bedenken, die die Umstellung auch bei uns begleitet haben - Zugang, Entlehnbedingungen und Literaturauswahl -, nicht bewahrheitet. Dieses Entgegenkommen ist nicht zu verwechseln mit professioneller Selbstaufgabe in Form von Liebedienerei: Im Sinn unseres Auftrags, Forschung und Lehre zu unterstützen, fokussieren wir uns darauf, dort großzügig zu agieren, wo es durch den Forschungsprozess und seine Ziele gerechtfertigt scheint.

Als Bibliothekar_innen sehen wir uns aber auch in der Rolle, zwischen den Nutzer_innengruppen und ihren jeweiligen Nutzungsinteressen zu vermitteln. Die Stellung der rund 800 Studierenden konnte daher nicht so bleiben, wie sie

7 Andrew Abbott: Digital Paper. A Manual for Research and Writing with Library and Internet Materials. Chicago: The University of Chicago Press 2014, S. 81. 
war. Das weitverbreitete Modell »Wochenendentlehnung als Gnadenakt« haben wir auf 7-Tage-Entlehnung bei einer Gesamtentlehndauer von einem Jahr umgestellt, was der bestmögliche Kompromiss zwischen Nutzbarkeit zu Hause für alle und schneller Verfügbarkeit bei Vormerkung ist. Die Anzahl der maximal entlehnbaren Werke wurde für alle Nutzer_innengruppen stufenweise von anfangs schlanken fünf auf 25 erhöht. Die großzügigere Entlehnung an Studierende hat keinerlei Konflikte mit den Institutsangehörigen erzeugt, was angesichts des jahrzehntelangen Vorbehalts gegenüber einer solchen Lockerung überrascht.

\section{Was durch Standortzusammenlegungen verloren geht}

Die Umwandlung der Institutsbibliothek Musikwissenschaft in eine Fachbereichsbibliothek blieb in einem Punkt hinter den Planungen zurück: Die angedachte räumliche Eingliederung in eine größere, fächerübergreifende Bibliothek musste aus Kostengründen unterbleiben. Das ist im Rückblick gut. Dass unsere Bibliothek gleichsam wie eine "Institutsbibliothek plus« benutzt werden kann, die Vorteile für Personal und Studierende zu verbinden sucht, verdankt sich auch ihrer unveränderten Größe. Eine größere Einheit hätte die Beibehaltung der genannten Freiheiten bis an die Grenze der Unmöglichkeit erschwert, da gerade Faktoren wie die Benutzung außerhalb der Öffnungszeiten eine Zuverlässigkeit voraussetzen, die auf einer persönlich verantworteten statt einer abstrakten Kollegialität basiert. Dieser Vorteil ist ein Effekt unserer spezifischen Größe, die damit zu einem Wert für sich wird. Dass er sich nicht unmittelbar in Zahlen ausdrücken lässt, heißt angesichts der Bedürfnisse von Geisteswissenschafter_innen an ihr grundlegendes Arbeitsinstrument Bibliothek nicht, dass er nicht wirken würde: Jeder Schritt, der sie von ihrer »idealen Norm« entfernt, senkt den Wirkungsgrad der eingesetzten Ressourcen, weil die Anzahl der möglichen Wissensgewinne eingeschränkt wird. Dass das nicht im Interesse der Auftraggeber sein kann, die nicht nur die Bibliotheken erhalten, sondern auch in die Forscher_innen und deren Arbeit investieren, sollte in Zusammenhang mit Fusionierungsdiskussionen immer wieder in Erinnerung gerufen werden. 


\section{Keine Außenseiter mehr. Die Fachbereichsbibliothek Ostasienwissenschaften}

Die Fachbereichsbibliothek Ostasienwissenschaften besteht seit dem 1. Jänner 2000. Sie entstand durch die Zusammenlegung der ehemaligen Fachbibliothek für Japanologie und Koreanologie sowie der Institutsbibliothek für Sinologie in Folge der räumlichen Vereinigung nach der Übersiedlung auf den Campus der Universität Wien (Altes AKH) und die Zusammenlegung der beiden Institute.

Die einzelnen Abteilungen der Fachbereichsbibliothek Ostasienwissenschaften haben ganz verschiedene Entstehungsgeschichten, was bis heute an den unterschiedlichen Strukturen zu sehen ist. Die ersten japanologischen Bestände gelangten bereits 1938 nach Wien, als Oka Masao (1898-1982), der 1939 der erste Leiter des Instituts für Japankunde werden sollte, eine umfangreiche Schenkung mitbrachte, die heute noch an dem Stempel »Institut für Japankunde - BaronMitsui-Stiftung« zu erkennen ist. Diese Bestände wurden erweitert, bis 1944 der Institutsbetrieb kriegsbedingt eingestellt werden musste und die Bibliotheksbestände ausgelagert wurden. Das Institut für Japankunde hörte im April 1945 auf zu bestehen, die Bibliothek wurde 1947 nach Wien zurückgebracht und dem Institut für Völkerkunde einverleibt. 1953 erhielt die Japanbibliothek einen eigenen kleinen Raum in der Stallburg, und erst dann konnte mit einer (bescheidenen) Aufstockung der Bestände begonnen werden. Das Institut für Japanologie wurde schließlich 1965 durch Herauslösung der Japan-Abteilung aus dem Institut für Völkerkunde gegründet und konnte damals eine Bibliothek übernehmen, die immerhin schon 9.500 Inventarnummern aufwies. Seit damals gibt es auch eine eigene Koreabibliothek, die dem Institut für Japanologie eingegliedert war. Der sinologische Bestand der Bibliothek, der in seinen Anfängen ebenfalls auf die Ausgliederung aus der Völkerkunde zurückgeht, wurde seit der Gründung des Instituts für Sinologie 1973 aufgebaut. Bis jetzt ist die Fachbereichsbibliothek Ostasienwissenschaften die einzige Bibliothek in Österreich, die systematisch Literatur in chinesischer, japanischer und koreanischer Sprache sammelt. 


\section{Schriftprobleme}

In den vergangenen zehn bis fünfzehn Jahren hat sich für die Fachbereichsbibliothek Ostasienwissenschaften eine Menge verändert. So gelang es, die Bibliothek nach und nach in die Universitätsbibliothek zu integrieren, angefangen mit der Katalogisierung über die elektronische Enlehnverbuchung bis zur Zeitschriftenverwaltung. Die große Hürde dabei war in erster Linie das Problem der Schriften. Im Gegensatz zu den meisten anderen Sprachen, die Buchstabenbzw. Silbenschriften verwenden, ist für das Chinesische, Japanische und Koreanische eine Transliteration nicht möglich. Wir verwenden Transkriptionen, die nicht eindeutig reversibel sind, da jedes Schriftzeichen mehrere Lesungen haben kann und andererseits jede Silbe in eine ganze Reihe (bedeutungstragender) Schriftzeichen rückübertragbar ist. In diesem Zusammenhang wurde der Autorin dieses Beitrages die Mitarbeit in der DIN-Arbeitsgruppe zur Umschrift des Japanischen ermöglicht. Weil es mit dem Bibliothekssystem BIBOS noch nicht möglich war, diese Schriften darzustellen, und bei einer ausschließlich auf Transkription basierenden Katalogisierung zuviel Information verlorengegangen wäre, wurden originalsprachliche Titel in allegro bzw. lidos katalogisiert und in einem Zettelkatalog zugänglich gemacht. Leider war es später nicht möglich, diese Daten wieder in das eigene Bibliothekssystem zu importieren, aber die alten Kärtchen dienen als Unterstützung bei der Retrokatalogisierung. Erst seit im Bibliothekssystem Aleph die Erfassung der CJK-Titel in Originalschrift möglich wurde, können auch die Bestände der Fachbereichsbibliothek Ostasienwissenschaften in den Online-Katalog der Universitätsbibliothek eingearbeitet werden. Anders als vielen anderen europäischen Bibliotheken, die Katalogisate zum Teil maschinell übernehmen oder gar nicht in einen Verbund integriert sind, ist es uns ein Anliegen, auch tatsächlich in "unserem» Verbund vertreten zu sein und die Titel sowohl in der Originalsprache als auch in der »im Fach « üblichen Transkription zu verzeichnen.

Nachdem in einem ersten Schritt zumindest die westlichsprachigen Bestände vollständig erfasst wurden, konnte schließlich auch die Fachbereichsbibliothek Ostasienwissenschaften mit der Entlehnungverbuchung über das Bibliothekssystem beginnen. Leider sind wir, was die CJK-Bestände betrifft, noch weit von einer Vollständigkeit entfernt, da die Retrokatalogisierung noch lange nicht abgeschlossen ist. Nicht im System verzeichnete originalsprachliche Titel müssen nach Bedarf schnell katalogisiert werden, um eine Entlehnung zu ermöglichen. Für die Zukunft hoffen wir, dass bald auch die originalsprachlichen Altbestände mit korrekten Transkriptionen erfasst werden können.

Nicht zuletzt konnte sich die Fachbereichsbibliothek aufgrund erhöhten Personaleinsatzes weiter entwickeln. Da hier drei völlig verschiedene Sprachen vertreten sind, sind auch drei Expert_innen notwendig, um einerseits die Lite- 
raturauswahl und -bearbeitung zu erledigen (nur die westlichsprachige Literatur wird weiterhin zentral erfasst) und andererseits Studierende und Institutspersonal fachgerecht zu betreuen. Auch unsere studentischen Mitarbeiter_innen sind "vom Fach«; ohne die entsprechenden Sprach- und Fachkenntnisse wäre auch die Arbeit am Infopult nur eingeschränkt möglich. Als erste erhielt die Japanologie eine Bibliothekarsstelle, als die Universität Wien eine zu Beginn von der Japan Foundation unterstützte Stelle weiterfinanzierte, so wie das später auch für die beiden anderen Abteilungen, mit anfänglicher Hilfe der Korea Foundation bzw. der Chiang Ching-kuo Foundation, erreicht wurde.

Ein großes Ziel für die kommenden Jahre ist die komplette Retrokatalogisierung (derzeit sind noch mehr als 40.000 Titel in japanischer, chinesischer und koreanischer Sprache nicht im Online-Katalog verzeichnet), aber was immer die Zukunft der Bibliothek bringen wird, werden wir gemeinsam bewältigen. 
Open-Access-Publikation im Sinne der CC-Lizenz BY-NC-ND 4.0

(c) 2019, Vandenhoeck \& Ruprecht GmbH \& Co. KG, Göttingen ISBN Print: 9783847110989 - ISBN E-Lib: 9783737010986 


\section{Eine neue Bibliothek - zwei alte Wurzeln. Die Fachbereichsbibliothek Osteuropäische Geschichte und Slawistik}

\section{Es waren einmal zwei Institutsbibliotheken ... Es waren einmal zwei Fachbereichsbibliotheken ...}

Doch im Jahr 2013 wurde aus diesen zwei altehrwürdigen Einrichtungen die neue, gemeinsame Fachbereichsbibliothek für Osteuropäische Geschichte und Slawistik. Obwohl die Fachbereichsbibliothek in der heutigen Form also noch keine sehr lange Geschichte hat, reichen ihre Wurzeln weit zurück. Am Anfang standen eine Instituts- und eine Seminarbibliothek - wer sich mit der Geschichte des Instituts für Osteuropäische Geschichte befasst ${ }^{1}$, wird sehen, dass es sogar um eine Buchsammlung herum gegründet wurde. Die Fachbereichsbibliothek ist daher, auch in ihrer heutigen, veränderten Form immer noch untrennbar mit den beiden Instituten und deren Geschichte verbunden. Auch am Buchbestand wird diese lange Geschichte sichtbar: Eines unserer ältesten vorhandenen Bücher De re militari von Robertus Valturius (1405-1475) stammt aus dem Jahr 1532 und gilt als das erste gedruckte Buch mit technischen Illustrationen. Es gibt seltene Bücher wie die Ausgabe der Ostroger Bibel von 1581 und mit wunderschönen Stichen illustrierte Werke wie die Historiae naturalis aus dem Jahr 1650 von Jan Jonston (1603-1675).

\section{Warum eine Zusammenlegung nicht nur viel Arbeit, sondern auch Sinn machte?}

Zum einen war seit der Übersiedlung auf den Campus der Universität Wien 1998 eine räumliche Nähe gegeben - wenn man vom Institut die Treppe hinaufkam oder über die Pawlatsche in den Bibliothekstrakt wechselte, ging man linkerhand in die eine, rechterhand in die andere Fachbereichsbibliothek.

1 Walter Leitsch, Manfred Stoy: Das Seminar für osteuropäische Geschichte der Universität Wien 1907-1948. Wien u. a.: Böhlau 1983. 
Zum anderen gibt es eine inhaltliche Nähe. Obwohl das Institut für Slawistik zur Philologisch-Kulturwissenschaftlichen Fakultät und das Institut für Osteuropäische Geschichte zur Historisch-Kulturwissenschaftlichen Fakultät gehört, gibt es zahlreiche Überschneidungen zwischen den beiden Fächern. Viele Studierende benötigen daher Bücher aus beiden Fachbereichen und empfinden es als Erleichterung, nur noch eine Bibliothek aufsuchen zu müssen.

Als die Zusammenlegung erstmals besprochen wurde, leitete Norbert Brien die Fachbereichsbibliothek Slawistik und Markus Stumpf die Fachbereichsbibliothek Osteuropäische Geschichte gemeinsam mit der Fachbereichsbibliothek Zeitgeschichte. Noch vor der Zusammenlegung im Sommer 2013 wurde die Autorin dieses Betrags im Rahmen einer kleinen Ausstellung an der Fachbereichsbibliothek Slawistik dem Institut als neue Leiterin vorgestellt und ordnungsgemäß mit einem Gläschen Wodka willkommen geheißen. Norbert Brien blieb auch nach seinem Wechsel an die Hauptbibliothek für die Buchauswahl und Sacherschließung des Fachbereichs Slawistik zuständig.

Das Wissen über die beiden Fachbereichsbibliotheken - Bestand, bisherige Abläufe, historische Gegebenheiten usw. - bestand allerdings auch vor Ort weiter. Aus der Fachbereichsbibliothek Slawistik brachte Krystyna Zalega ihre bisherigen Erfahrungen ein, aus der Fachbereichsbibliothek Osteuropäische Geschichte Volkmar Neuer. Aus der Abteilung Formalerschließung der Hauptbibliothek wechselte Elisa Nemetz für 20 Wochenstunden an die Fachbereichsbibliothek, womit das Kernteam komplett war.

\section{Signaturen, Platzprobleme und andere Herausforderungen}

Bevor die gemeinsame Fachbereichsbibliothek eröffnet werden konnte, mussten die Bücher umgeräumt werden. Schnell war klar, dass das ohne Unterstützung nicht möglich sein würde. Mithilfe freier studentischer Mitarbeiter und einer Transportfirma wurde beinahe jedes unserer 210.000 Bücher umgestellt. Der so gewonnene Platz sollte für zehn Jahre reichen - eine Einschätzung, die sich in den letzten fünf Jahren bestätigt hat.

Die Räumlichkeiten haben wir schnell besser kennengelernt. Als nach einer regenreichen Zeit ein Wasserfleck an der Decke zu sehen war, stellte sich heraus, dass die Regenrinnen am Campus innerhalb des Daches geführt werden und es in der Bibliothek schnell bemerkbar wird, wenn sie defekt sind. (Es genügt für die Entstehung von Wasserflecken an der Decke aber auch, die Fenster im Dachstuhl offen zu lassen, wie bei anderer Gelegenheit bemerkt wurde.) Die Decke fällt einem allerdings nicht gleich auf den Kopf, wenn sie beginnt, sich abzulösen, und so erfährt man auch, was mit einer »abgehängten« Decke gemeint ist. 
Auch nach Bewältigung der eigentlichen Zusammenlegung gibt es noch einige Probleme, die manchmal aber tatsächlich Möglichkeiten sind, um Wege zu finden, die Bibliothek weiter zu verbessern. Verständlicherweise ist es für viele schwer, sich beim ersten Besuch in der Fachbereichsbibliothek zurechtzufinden. Das lässt sich unter anderem mit den historisch gewachsenen Signaturen erklären und ist nicht leicht zu ändern und zu bereinigen.

In den nächsten Jahren soll daher zunächst an einer übersichtlichen Aufstellung der Zeitschriften gearbeitet werden. Früher waren viele Zeitschriften an beiden Institutsbibliotheken vorhanden, weshalb es viele Dubletten gibt. Bereinigt man diese, ist es möglich, den Platz in der Bibliothek besser zu nutzen. Die Signaturen sind vor allem im Bereich Slawistik aufgrund unterschiedlicher früherer Standorte uneinheitlich und nicht aussagekräftig. Daher sind diese Zeitschriften auch nicht nach Signaturen, sondern nach Preußischen Instruktionen aufgestellt. Bei der Sprachenvielfalt der Fachbereichsbibliothek bedeutet dies, dass in allen Titeln, egal welcher slawischen Sprache, zuerst das unveränderte Hauptwort bestimmt werden muss, bevor die Zeitschrift gefunden werden kann. Mit einer vollständigen Katalogisierung und neuen Signaturen für die Zeitschriften ist auch eine zukünftige Auslagerung in ein Depot eine Perspektive.

\section{Die lieben Kolleg_innen ...}

Derzeit ist die Fachbereichsbibliothek noch nicht vollständig retrokatalogisiert, sodass die Aufstellung des Nominalkatalogs im Lesesaal nach wie vor ein Muss ist. Ein schwerer Schlag war es nicht nur für das Projekt Retrokatalogisierung, sondern für das gesamte Team, als Brigitte Maresch (1946-2016) im Jahr 2016 überraschend verstarb. Trotz bereits angetretenem Ruhestand kam sie weiterhin in die Fachbereichsbibliothek um zu katalogisieren und - fast noch wichtiger stärkte unser Team und den Teamzusammenhalt jeden Mittwoch mit Kaffee und Kuchen.

Ende 2018/Anfang 2019 fand in der Fachbereichsbibliothek eine weitere große Veränderung statt: Ende August ging unsere Kollegin Krystyna Zalega in Pension und im Februar 2019 unser Kollege Norbert Brien. Neben der Aufstockung von Elisa Nemetz auf 35 Wochenstunden sind nun insgesamt fünf Studierende mit 6-8 Wochenstunden an der Fachbereichsbibliothek tätig. Waren sie früher zur Unterstützung des Kernpersonals da, ist der Betrieb nun ohne sie nicht mehr aufrecht zu halten. 


\section{Von Vögeln und anderen Benutzer_innen}

Fast alle Veränderungen der letzten Jahre haben die Nutzer_innen entweder freudig begrüßt oder (notgedrungen) mitgetragen. Besonders willkommen war der Wechsel von einer Präsenzbibliothek zur Möglichkeit einer 7-Tage-Entlehnung sowie die Ausweitung des Freihandbereichs im Zuge der Zusammenlegung. Vermisst wird aber auch Jahre nach der Zusammenlegung gelegentlich noch der Zugang zur Fachbereichsbibliothek vom Institut für Slawistik über die Pawlatsche - auch wenn der neue Zugang nun direkter und barrierefrei ist. Den Garderobenkästen, für die man immer an 1- oder 2-Euro-Stücke denken musste, trauert jedoch niemand nach. Dafür werden wir gelegentlich von unseren Benutzer_innen gefragt, ob sie den Schlüssel behalten dürfen, wenn sie kurz rauchen gehen. Sogar die am Campus wohnenden Vögel fühlen sich in der Fachbereichsbibliothek wohl und haben dem Lesesaal schon mehrere Besuche abgestattet.

\section{War - Ist - Wird sein ...?}

Die Fachbereichsbibliothek Osteuropäische Geschichte und Slawistik hat in vielen Dingen zwei Gesichter. Sie ist auch nach über fünf Jahren noch eine geteilte Bibliothek was Signaturen, Aufstellung und Budget der zwei Fachbereiche betrifft. Auf der anderen Seite aber hat sich nach fünf Jahren ein Team gefunden und gebildet, das als eines agiert, kommuniziert und aufeinander eingespielt ist. Wir haben eine reiche Geschichte und sind dennoch eine neue Fachbereichsbibliothek, wir haben historisch gewachsene Probleme vor allem im Bereich der Signaturen, aber dennoch sind wir immer bemüht, uns den modernen Gegebenheiten anzupassen und den Wünschen der Benutzer_innen gegenüber offen zu sein. Auch in Zukunft werden wir uns bemühen, dass dieser Spagat weiterhin und immer noch besser gelingt. 


\section{Arzneistoffe, Arzneipflanzen, Public Health - Von der Inkunabel zur Online-Suchmaschine. 25 Jahre Fachbereichsbibliothek Pharmazie und Ernährungswissenschaften im Universitätszentrum Althanstraße}

\section{Gründung und Beginn}

Im September 1994 übersiedelten alle bis dahin in der Währinger Straße verstreuten Institute der Pharmazie (Pharmazeutische Chemie, Pharmakognosie, Pharmazeutische Technologie, Pharmakologie und Toxikologie) zusammen mit dem Institut für Ernährungswissenschaften in das neu errichtete »PharmazieZentrum« (nunmehr anglisiert: PharmaCenter) im Universitätszentrum Althanstraße, ${ }^{1}$ Haus II (UZA-II). Gleichzeitig wurde durch Zusammenführung der bisherigen Institutsbibliotheken die »Fachbibliothek für Pharmazie und Ernährungswissenschaften « errichtet, als erste (und bisher einzige) dieser Fächer in Österreich. ${ }^{2}$

Ursprünglich war zwar eine gemeinsame »Fachbereichsbibliothek Erdwissenschaften und Pharmazie« über zwei Ebenen hinweg im zentralen Hörsaaltrakt geplant gewesen, doch mitten in der Bauphase erfolgte aufgrund von massiven Einwänden der pharmazeutischen Fachvertreter die funktionale Aufspaltung in zwei autonome Fachbibliotheken. Da eine bauliche Trennung nun aber nicht mehr möglich war, wurden der Fachbereichsbibliothek Erdwissenschaften die Ebene 5 und der Fachbereichsbibliothek Pharmazie und Ernährungswissenschaften die Ebene 4 zugewiesen. Daraus ergaben sich nicht nur ein gemeinsamer Bibliothekseingang, ein gemeinsames Infopult und identische Öffnungszeiten, sondern auch eine bis heute bestehende sehr heterogene Aus-

1 https://de.wikipedia.org/wiki/Universit\%C3\%A4tszentrum_Althanstra\%C3\%9Fe [Letzter Zugriff: 10.01.2019].

2 Siehe dazu Johann Jurenitsch, Claudia Müller, Kurt Schneider, Wolfgang Kubelka: 200 Jahre Pharmakognosie in Wien. Eine Wissenschaft im Dienst der Arzneimittelsicherheit. Wien: Facultas 1998, sowie Kurt Schneider: Bibliothek der Institute für Pharmakognosie und Pharmazeutische Technologie an der Universität Wien. In: Handbuch der historischen Buchbestände in Deutschland, Österreich und Europa. Hg. von Bernhard Fabian. Digitalisiert von Günter Kükenshöner. Hildesheim: Olms Neue Medien 2003, http://fabian.sub.uni-goettin gen.de/fabian?Pharmakognosie_(Wien) [Letzter Zugriff: 10.01.2019]. 
stattung dieser beiden Bibliotheken mit Lese- und Online-Katalog-Plätzen, Regal- und Kompaktusflächen. Auch der Personaleinsatz mit ursprünglich zwei Leitern und zwei Mitarbeitern, inzwischen verstärkt durch fünf studentische Mitarbeiter_innen zur Abdeckung der erweiterten Abendöffnungszeiten, erfolgt kooperativ.

Wegen des komplexen Aufbaus des Universitätszentrums Althanstraße, das sich auf einer langen »Platte« über den Gleisanlagen der Franz-Josefs-Bahn ohne direkten Zugang auf Straßenniveau befindet, stellt die formale Adresse "Althanstraße 14« eine reine Postanschrift dar und führt direkt in den verirrungsanfälligen »Untergrund der Tiefgaragen".

Zur Illustration der heute kaum mehr vorstellbaren damaligen bibliothekarischen Situation einige Schlaglichter:

- Die Freischaltung des FAX-Gerätes auch für internationale Verbindungen (!) erforderte eine langwierige gemeinsame Genehmigungsprozedur durch die damalige Universitäts- sowie die Bibliotheksdirektorin.

- Als "Sonderausstattung" wurde in einem Leiterbüro eine zweite Telefonsteckdose installiert, um technisch zukunftssicher für eine eventuelle Informationsvermittlungsstelle (IVS) gerüstet zu sein.

- IT State of the Art waren Windows 95, GOPHER (abgelöst durch das World Wide Web), PINE via Telnet (abgelöst durch MS Outlook \& Webmail) sowie 14-Zoll-S/W-Monitore und 9-Nadel-Drucker mit Endlospapier für die Online-Katalog-Geräte. Der getrennt ausgeschilderte Bereich "AV-Medien" (heute Lern- und Kopierzentrum) bestand lediglich aus einem Videowagen für Monitor und Videorecorder.

- Bibliothekarisch aktuell waren das EDV-gestützte »Bibliotheksorganisationssystem«(BIBOS), die »Regeln für die Schlagwortkatalogisierung ( RSWK) und die "Österreichische Zeitschriften-Datenbank« (ÖZDB), daneben Mikrofiche-Tröge und die alten Zettelkataloge der ehemaligen Institutsbibliotheken nach unterschiedlichen hauseigenen Regeln.

\begin{abstract}
Aktuell
Die Bibliothek umfasst derzeit ca. 45.000 Bände (davon 6.500 Hochschulschriften), die fast ausschließlich im Freihandbereich nach einer hauseigenen, eng an die Regensburger Verbundklassifikation (RVK) angelehnten Systematik aufgestellt sind. Der gesamte Präsenz-Bestand ab 1910 ist inzwischen dank mehrerer Retro-Projekte und mit Hilfe des Teams Integrierte Medienbearbeitung Fachbereichsbibliotheken online erfasst.
\end{abstract}




\section{Sondersammlungen}

$\mathrm{Zu}$ erwähnen sind hier der umfangreiche Bestand an historischen Arzneibüchern (Pharmakopöen), die Sammlung großformatiger historischer Druckgraphiken (Naturselbstdrucke und handkolorierte Pflanzentafeln) sowie eine Kollektion handgeschriebener Heilwasseranalysen mit entsprechenden KurorteProspekten aus dem Gebiet der ehemaligen österreichisch-ungarischen Monarchie. Aus diesem Sonderbestand wurden bereits mehrfach interessante »Objekte des Monats" präsentiert: 2012 »Riesenschachtelhalm als Naturselbstdruck«, 2016 »Weinrebe Sammlung PACH«, 2017 »Widmungskassette Prof. Vogl« und zuletzt 2018 »Broschüre und Analysenblätter Heilbad Trencsénteplicz«.

\section{Veranstaltungen}

Ganz besonders gut angenommen werden die angebotenen Lesungen, Buchpräsentationen und Ausstellungen, die als »Buffet-gestützte Social Events« den Fachvertretern und externen Gästen Gelegenheit geben, in entspannter Atmosphäre die Fachbereichsbibliothek einmal ganz anders zu erleben und ohne Zeitdruck Gespräche mit Kolleg_innen auch anderer Fachbereiche zu führen.

\section{Nutzung}

Unsere Benutzer_innen setzen sich primär aus den ca. 2.500 Studierenden des Faches Pharmazie, 3.000 Studierenden des Faches Ernährungswissenschaften sowie den etwa 500 internen und externen Lehrenden und Wissenschafter_innen zusammen. Daneben ist aber auch die Versorgung der in der pharmazeutischen Praxis (Apothekerkammer, Apotheken, Pharmazeutische Industrie, Agentur für Arzneimittelsicherheit und Ernährung, Untersuchungslaboratorien etc.) tätigen Absolvent_innen mit relevanten, speziell auch nur in gedruckter Form verfügbaren Medien immer wichtiger geworden.

Neben allgemeinen Einführungen in die Benutzung der Fachbereichsbibiothek für interne und externe (z. B. Fachhochschulen) Nutzer_innen werden auch zielgerichtete Veranstaltungen in Zusammenarbeit mit den jeweiligen Studienprogrammleitungen angeboten (Schulung der Mentor_innen der Ernährungswissenschaften, Mitwirkung bei pharmazeutischen Pflichtvorlesungen etc.). 


\section{Ausblick}

Auch wenn Ausmaß und Tempo der Digitalisierung speziell in den Lebenswissenschaften während der letzten 15 Jahre mit der Verfügbarkeit von WebDatenbanken und dem fast vollständigen Ersatz der gedruckten Zeitschriften durch E-Journals beeindruckend waren, so scheint sich im Bereich der E-Books eine doch etwas andere Entwicklung abzuzeichnen. Einerseits sind gedruckte Lehrbücher für Studierende nach wie vor unverzichtbar, wie die Rückmeldungen seitens der Studentenvertretungen zeigen; andererseits erschwert die (Über)Fülle an lizensierten E-Book-Paketen zusammen mit deren gelegentlich nicht dauerhaften Verfügbarkeit und den als hinderlich empfundenen Restriktionen im Download-Volumen die gewünschte höhere Akzeptanz der E-Books. Hier werden wir Bibliothekar_innen verstärkt gefordert sein, als fachlich kompetente »Medien-Gatekeeper« unterstützend tätig zu werden. Dem steht allerdings die seit Inkrafttreten des UG 2002 an vielen Universitäten zunehmende Tendenz entgegen, hochqualifizierte unbefristete Vollzeitstellen entweder gar nicht oder durch befristete und geringer qualifizierte Teilzeitstellen nachzubesetzen sowie den Einsatz geringfügig beschäftigter studentischer Hilfskräfte immer mehr auszuweiten. Dies mag zwar kurzfristig Personalkosten einsparen helfen, hat aber langfristig durch den unvermeidlichen Verlust an Fachkompetenz fatale Folgen für die mühsam und langwierig erworbene Akzeptanz von uns Bibliothekar_innen als Ansprechpartner "auf fachlicher Augenhöhe« für Fakultät, Departments und Fachvertreter!

Videant consules ne quid detrimenti res bibliothecalis capiat! 


\section{Vor Gericht und auf hoher See ... Die Fachbereichsbibliothek Philosophie}

Wenn man im Jahr 2018 die Leitung einer Fachbereichsbibliothek antritt, gleicht dieses Manöver der Übernahme des Steuerruders mitten auf hoher See: Man muss das Ruder fest in der Hand halten und darf den Kurs nicht aus den Augen verlieren ...

Es gilt, den Schatz der Philosophen sicher durch die Zeiten zu manövrieren. Im Zentrum der Arbeit der Philosophinnen und Philosophen stehen die Überlegungen ihrer Vorgänger. Das Denken benötigt immer wieder Impulse: So bezieht man Gedanken und Zitate der Vergangenheit in das gegenwärtige Denken mit ein, um daraus neue Erkenntnisse zu gewinnen. Das Wiederauffinden von Texten und Überlegungen ist ein wichtiger Bestandteil des wissenschaftlichen Arbeitens. So sind die Philosophinnen und Philosophen immer wieder mit ihrer Geschichte konfrontiert, und die Geschichte wird Teil ihrer Reflexion. Genauso wichtig wie das genaue wissenschaftliche Recherchieren ist somit auch die Zugänglichkeit der Texte. Es müssen die wichtigen Gedanken der Vergangenheit zugänglich gehalten werden. Dies ist besonders von Bedeutung im $\mathrm{Zu}$ sammenhang mit E-Book-Paketen: Die maßgebliche Literatur muss nachhaltig zur Verfügung stehen.

Der Kurs des Schiffes, um bei diesem Vergleich zu bleiben, ist also die Gewährleistung der Zugänglichkeit. Die zentrale Frage im Jahr 2018 lautete daher, wie man elektronische und analoge Medien zu einem sinnvollen Zusammenspiel vereint. Einerseits ist der Standort der Fachbereichsbibliothek von großer Bedeutung für das Institut und das wissenschaftliche Arbeiten vor Ort, andererseits gilt es, große E-Book-Pakete zugänglich zu machen und auch zugänglich zu halten. Die Gratwanderung beginnt genau an dieser Stelle.

Das große Dilemma, das sich hier auftut, ist die Frage, welches E-Book-Paket und welche Datenbank man bevorzugt ankauft und wie man die unterschiedlichen Ankaufswünsche bewertet und gewichtet. Eine genaue Analyse der verschiedenen Ankaufsmöglichkeiten ist hier in Zukunft besonders gefragt. Eine Bedarfsanalyse der wissenschaftlichen Infrastruktur ist ein wichtiger Bestand- 
teil der täglichen Arbeit geworden. Digitale innovative Lösungen der Präsentation und Vermittlung werden in Zukunft von großer Bedeutung sein.

In der Bibliothek muss man vermehrt darauf achten, alle Ebenen der Wahrnehmung anzusprechen. Da die Benutzerinnen und Benutzer unterschiedliche Zugangsweisen und Bedürfnisse haben, müssen verschiedene Einstiege und Möglichkeiten parallel angeboten werden. Die Bedeutung des persönlichen Gesprächs kann hier nicht genug betont werden - es macht den entscheidenden Unterschied aus. Erst wenn alle Ebenen des Aufnehmens, Mitnehmens und Verarbeitens angesprochen werden, macht der Besuch vor Ort Freude.

Der Bibliotheksbesuch muss auch in Zeiten des elektronischen Fortschritts attraktiv und inspirierend bleiben. Mit Hilfe von QR-Codes kann auf viele Informationen, wie zum Beispiel Neuerscheinungen, hingewiesen werden. Im Eingangsbereich wäre die Suche am Touchscreen ein ansprechender innovativer Einstieg, um die Studierenden z. B. mit verschiedenen philosophischen Zeitschriften vertraut zu machen.

Von zentraler Bedeutung ist das konkrete Eingehen auf die Wünsche der Studierenden und Forschenden. Das elektronische Wunschbuch erfreut sich in diesem Zusammenhang großer Beliebtheit. Die Fachbereichsbibliothek kann hier eine ganz wichtige Rolle im Leben der Studentinnen und Studenten spielen, indem sie einen breiteren und umfassenderen Zugang zu den Ressourcen vermittelt und als wichtige Anlaufstelle mit neuen Ansprechpersonen fungiert.

Das Wissen um die Mechanismen wissenschaftlichen Arbeitens einerseits und der Überblick über die Zusammenhänge andererseits gehört neben dem Verfügbarhalten der Bestände und dem Vermitteln von Informationskompetenz zu den Kernkompetenzen der Bibliothekarinnen und Bibliothekare. Repositorien werden in Zukunft eine wichtige Rolle spielen und es wird Aufgabe der Fachbereichsbibliothek sein, hier die notwendigen Informationen am Institut zu liefern und als Anlaufstelle zu fungieren.

Der Standort am Institut dient daher als Anlaufstelle für Fragen und Probleme vor Ort. Besonders wichtig ist dabei die Vernetzung der Personen am Institut. Gleichzeitig stellt die Fachbereichsbibliothek aber auch den Kontakt zu den verschiedenen Bereichen und Fachexperten der Dienstleistungseinrichtung Bibliotheks- und Archivwesen mit ihren vielfältigen Services her. Veranstaltungen mit Vorträgen von Kolleginnen und Kollegen aus den zentralen und forschungsunterstützenden Bereichen der Universitätsbibliothek können eine wertvolle Ergänzung zu den vorhandenen Informationswegen darstellen.

Die Mailinglisten des Instituts geben Aufschluss über die aktuellen Forschungsfelder und Veranstaltungen, Führungen vor Ort dienen dem persönlichen Kontakt und sollen ein Interesse für zukünftige Bibliotheksbesuche wecken. 
Folder spielen nach wie vor eine große Rolle, um Informationen und Hilfestellungen anzubieten. Auch hier muss auf allen Wegen der Informationsvermittlung gearbeitet werden - Verweise auf die Website und auf weiterführende Informationen sind unerlässlich.

Die kommende Generation von Philosophinnen und Philosophen wird Gericht halten über unser gegenwärtiges Handeln, das hoffentlich auch die zukünftige Generation an Wissenschaftern einschließt.

Es bleibt noch die Sehnsucht - die Sehnsucht nach Überblick und Zugänglichkeit. Und die Erkenntnis, dass es von größter Bedeutung ist, gut ausgebildete Mitarbeiterinnen und Mitarbeiter zu haben, die mit Leidenschaft und Hingabe jeden Tag dieses Ziel verfolgen. 
Open-Access-Publikation im Sinne der CC-Lizenz BY-NC-ND 4.0

(c) 2019, Vandenhoeck \& Ruprecht GmbH \& Co. KG, Göttingen ISBN Print: 9783847110989 - ISBN E-Lib: 9783737010986 


\section{»Die Bibliothek ist eine Lernstätte, nicht ein Lager für Bücher «'. Die Fachbereichsbibliothek Psychologie}

Eine Institutsbibliothek gab es seit der Gründung des Psychologischen Instituts im Jahr 1922, das aus einer Lehrkanzel am Philosophischen Institut hervorgegangen war. Das Psychologische Institut war ursprünglich im Palais Epstein, dem damaligen Gebäude des Stadtschulrates, untergebracht. Heute belegt die sehr stark expandierende Fakultät für Psychologie fast das gesamte "Alte Institutsgebäude« in der Liebiggasse 5 - gegenüber dem »Neuen Institutsgebäude«.

Im Jahr 1984 wurden die Bibliotheksbestände der Institutsbibliothek und Bestände des Instituts an weiteren Standorten, die bis dahin von einzelnen Lehrkanzeln am Institut für Psychologie verwaltet worden waren, als »Fachbibliothek Psychologie« zu einer organisatorischen Einheit zusammengefasst. Diese Standorte wurden bei der letzten Übersiedlung der Bibliothek im Jahr 2002 auch räumlich integriert. Die zur Verfügung stehenden Räumlichkeiten waren allerdings schon beim Bezug des neuen Standorts für die Fachbibliothek zu klein.

Die Fachbereichsbibliothek Psychologie bietet Informationen für Studium, Lehre und Forschung für das Fach Psychologie. Diese »Informationen« beinhalten die physischen und elektronischen Ressourcen, umfassen aber auch Informationsangebote, die Benutzer_innen dabei unterstützen, sich in der Fülle der Informationen zurechtzufinden und die zu einem kompetenten Umgang mit digitalen Informationen anleiten sollen. Die Fachbereichsbibliothek Psychologie versteht sich seit vielen Jahren als »Teaching Library $\aleph^{2}$. In den Anfängen ging es dabei um die Förderung der »Bibliothekskompetenz«. Im Zuge der Entwicklung

1 Melvil Dewey (1876), zitiert nach Mario Hütte: Zur Vermittlung von Informationskompetenz an Hochschulbibliotheken - Entwicklung, Status quo und Perspektiven. Master's Thesis. Köln: Fachhochschule Köln 2016. Siehe: http://eprints.rclis.org/8824/1/MT-_Mario-_Huette. pdf [Letzter Zugriff: 16.09.2019].

2 Als »Teaching Library« bezeichnet man eine Bibliothek, »die das Lehren und Lernen als eine Kernaufgabe ansieht « (nach: Claudia Lux, Wilfried Sühl-Strohmenger: Teaching Library in Deutschland. Vermittlung von Informations- und Medienkompetenz als Kernaufgabe für Öffentliche und Wissenschaftliche Bibliotheken. Wiesbaden: Dinges und Frick 2004, S. 17). 
zu einer hybriden Bibliothek stand zunächst die Motivation im Vordergrund, durch das Angebot geeigneter Schulungsmaßnahmen die optimale Nutzung der digitalen Bibliothek, also das kompetente Recherchieren mit den zur Verfügung stehenden Online-Tools und die bestmögliche Nutzung des elektronischen Bestandes, zu fördern. In den letzten Jahren hat sich der Focus auf die Vermittlung von "Informationskompetenz ${ }^{3}{ }^{2}$ verlagert. Dabei orientiert sich die Praxis an Theorien und Konzepten aus dem Bereich der Bibliotheks- und Informationswissenschaft, z.B. dem Framework der Association of College \& Research Libraries $^{4}$, aber auch an Publikationen aus dem Fach Psychologie zu diesem Thema, wie den 2013 von der American Psychological Association formulierten Lernzielen für das Psychologiestudium ${ }^{5}$ oder Forschungsergebnissen zur Vermittlung von Informationskompetenz (z. B. Rosman, Mayer \& Krampen, 2015, zum Einfluss verschiedener Instruktionsarten auf den Lernerfolg). ${ }^{6}$

Es wurde ein Konzept zur Vermittlung von Informationskompetenz im fachlichen Kontext von der Autorin dieses Beitrags, die von 1984 bis Februar 2019 Leiterin der Fachbereichsbibliothek Psychologie war, entwickelt und praktisch umgesetzt. Die Angebote umfassen eigenständige Veranstaltungen für Zielgruppen mit unterschiedlichem Wissensstand, Unterrichtseinheiten zur Literatursuche im Rahmen anderer Lehrveranstaltungen an der Fakultät für Psychologie und »betreutes Recherchieren«, das in der Fachbereichsbibliothek von Tutor_innen derselben angeboten wird. Die Website der Fachbereichsbibliothek bietet eine kommentierte Übersicht zur Recherche für das Fach Psychologie, Anleitungen zur Literatursuche, eine fachbezogene Linksammlung und Hinweise zu den Richtlinien zur Manuskriptgestaltung. Auf der Website der

3 Informationskompetenz umfasst »das Erkennen des jeweiligen Informationsbedarfs, die Formulierung von Suchanfragen, das Wählen geeigneter Zugangswege, die Bewertung von Information, die Integration von Wissen in den eigenen Wissensbestand, die zieladäquate Nutzung der Information und die ethische und gesetzeskonforme Nutzung." Michaela Zemanek: Informationskompetenz in Österreich. In: Wilfried Sühl-Strohmenger (Hg.): Handbuch Informationskompetenz. Berlin; Boston: De Gruyter Saur 2011, S. 498-531, hier S. 499. https://doi.org/10.1515/9783110255188.498 [Letzter Zugriff: 11.09.2019].

4 Association of College \& Research Libraries: Framework for information literacy for higher education, 2015. Verfügbar unter http://www.ala.org/acrl/sites/ala.org.acrl/files/content/is sues/infolit/Framework_ILHE.pdf [Letzter Zugriff: 17.01.2019].

5 American Psychological Association: APA Guidelines for the Undergraduate Psychology Major. Version 2.0. Washington, DC: Author 2013, Siehe: http://www.apa.org/ed/precollege/ about/psymajor-guidelines.pdf [Letzter Zugriff: 17.01.2019].

6 Tom Rosman, Anne-Kathrin Mayer, Günter Krampen: A longitudinal study on informationseeking knowledge in psychology undergraduates: Exploring the role of information literacy instruction and working memory capacity. Computers \& Education, 96, (2016), S. 94-108. Siehe: https://doi.org/10.1016/j.compedu.2016.02.011 [Letzter Zugriff: 11.09.2019]. 
Fachbereichsbibliothek findet sich auch eine "virtuelle Führung«, die eine zeitund ortsunabhängige Möglichkeit bietet, die Bibliothek kennenzulernen. ${ }^{7}$

Parallel zu den Angeboten der Fachbereichsbibliothek Psychologie unterrichtet die Autorin dieses Beitrags seit vielen Jahren in wechselnden Lehrveranstaltungsformaten "Wissenschaftliches Arbeiten" an der Fakultät für Psychologie. Die Tutor_innen der Fachbereichsbibliothek bieten für die Studienanfänger_innen begleitende Tutorien an.

Auf Anregung der Fakultät erstellte die Autorin dieses Beitrags im Jahr 2017 einen Online-Kurs zur Informationskompetenz in Moodle, der Lernplattform der Universität Wien, mit dem Ziel, den Studierenden zeit- und ortsunabhängig nutzbares Lernmaterial zur Verfügung zu stellen und ihnen auch die Möglichkeit zu geben, ihr Wissen in Bezug auf Informationskompetenz zu testen. Somit besteht für das Fach Psychologie an der Universität Wien ein umfassendes Angebot zur Informationskompetenz.

Das Institut für Psychologie unterstützte die Aktivitäten zur Förderung von Informationskompetenz, indem es der Fachbereichsbibliothek für viele Jahre Studienassistent_innen bzw. Tutor_innen zur Verfügung stellte. Diese Stellen sind mittlerweile organisatorisch der Bibliothek direkt zugeordnet.

Nicht zuletzt verdankt die Fachbereichsbibliothek Psychologie die Reichhaltigkeit ihres Angebotes auch der wohlwollenden Unterstützung der Bibliotheksdirektorin Maria Seissl, die die Erweiterung des Aufgaben- und Serviceportfolios der Universitätsbibliothek Wien stets im Blick hat. »Viel mehr als früher « $^{8}$ gilt als Leitsatz daher auch für die Fachbereichsbibliothek Psychologie.

7 https://bibliothek.univie.ac.at/fb-psychologie/Virtuelle_Bibliotheksfuehrung_FB_Psycho logie/.

8 Maria Seissl: Viel mehr als früher! Das Aufgaben- und Serviceportfolio der Universitätsbibliothek der Universität Wien. In: Die Bibliothek in der Zukunft: Regional - Global: Lesen, Studieren und Forschen im Wandel. Festschrift für Hofrat Dr. Martin Wieser anlässlich seiner Versetzung in den Ruhestand. Innsbruck: Innsbruck University Press 2015, S. 33-48, hier S. 1. Siehe: https://core.ac.uk/download/pdf/33186475.pdf [Letzter Zugriff: 16.01.2019]. 
Open-Access-Publikation im Sinne der CC-Lizenz BY-NC-ND 4.0

(c) 2019, Vandenhoeck \& Ruprecht GmbH \& Co. KG, Göttingen ISBN Print: 9783847110989 - ISBN E-Lib: 9783737010986 


\section{»Kaschmir «-Parkett und orange Kompaktanlagen. Die Fachbereichsbibliothek Publizistik- und Kommunikationswissenschaft und Informatik}

Die Fachbereichsbibliothek Publizistik- und Kommunikationswissenschaft und Informatik geht in ihrer heutigen Form auf das Jahr 2012 zurück. Wo jahrzehntelang eine Baulücke war, ziert seit jenem Jahr ein neues Gebäude die Währingerstraße 29, das zum Standort der aus zwei Fachbereichsbibliotheken zusammengelegten Fachbereichsbibliothek wurde. Sie ist gemeinsam mit der Fakultät für Informatik und dem Institut für Publizistik- und Kommunikationswissenschaft eingezogen. Als »innovativen Hintergedanken « lobte der damals amtierende Wissenschaftsminister Karlheinz Töchterle das Projekt, die beiden Fachgebiete unter einem gemeinsamen Dach zu beherbergen.

Dieses Projekt wurde im Namen des Bauherrenpreises 2012 als eines der drei besten Bau-Projekte in Wien nominiert. Die Errichtungsdauer des insgesamt neun Stockwerke umfassenden Bauwerks betrug zwei Jahre. Planung und Vorbereitung - auch seitens der Bibliothek - nahmen jedoch weit mehr Zeit in Anspruch. So wurde der gesamte zu übersiedelnde Buchbestand bearbeitet, die Studien des der Bibliothek zugehörigen Ernest Dichter-Archivs ${ }^{1}$ sortiert und in säurefreie Archivboxen umgebettet. Zudem wurden alle Informatik-Bücher umsigniert, um am neuen Standort Einheitlichkeit und leichtere Auffindbarkeit $\mathrm{zu}$ ermöglichen. Dafür war eine umfassende Zusammenarbeit der beiden Fachbereichsbibliotheken nötig, die sehr gut funktionierte. Darüber hinaus mussten neben der Dienstleistungseinrichtung Raum- und Ressourcenmanagement der Universität auch mit dem Architektenteam intensive Gespräche geführt werden. Anfängliche Missverständnisse und Informationslücken (so lautete eine der Fragen der Architekten »Wozu braucht eine Bibliothek Büros?«) konnten konstruktiv und mit Unterstützung der Bibliotheksdirektion geklärt werden. Das Ergebnis kann sich durchaus sehen lassen: Die Bibliothek ist auf drei Stockwerke verteilt. Das Erdgeschoß sowie das erste Untergeschoß sind tageslichtdurchflutet und mit Parkettboden, der den Namen »Eiche Kaschmir« trägt,

1 https://bibliothek.univie.ac.at/sammlungen/ernest_dichterarchiv.html [Letzter Zugriff: 21.08.2019]. 
ausgestattet. Im Erdgeschoss ist ein heller Lesesaal untergebracht, der den Benutzer_innen 70 mit Steckdosen ausgestattete Leseplätze sowie fünf PCs für ihre Recherchen und ihr Studium bietet. Im ersten Untergeschoss befindet sich neben einer Leselounge mit roten Sofas der Kopier- und Scanbereich. Da Nachhaltigkeit und Ressourcenschonung ein großes Anliegen des Teams der Fachbereichsbibliothek sind, wurde ein hochwertiger Buchscanner anstelle eines weiteren Kopiergerätes aufgestellt und ist aufgrund seiner kostenlosen Nutzung entsprechend beliebt. Ebenfalls beherbergt das erste Unterschoss den regelmäßig gebuchten und ebenfalls sehr beliebten "Gruppenarbeitsraum «, in dem sechs Personen für gemeinsames Arbeiten Platz finden. Im zweiten Untergeschoß dominiert die Farbe Orange - sowohl der Bodenbelag als auch die Kompaktanlagen sind in leuchtendem Orange gehalten und lassen dadurch kein Kellergefühl im herkömmlichen Sinn aufkommen.

Die Buchbestände aus dem Fachbereich »Informatik" gehörten bis zur Zusammenlegung zur damaligen Fachbereichsbibliothek Mathematik, Statistik und Informatik, welche auf mehrere Standorte aufgeteilt war. Vor der Übersiedlung und Zusammenlegung gab es eine Fachbereichsbibliothek Publizistikund Kommunikationswissenschaft, welche in einem viel zu kleinen Gebäude in der Schopenhauerstraße 32 untergebracht war. Für beide Fachbereichsbibliotheken war die damalige Situation aus diesen Gründen sehr unbefriedigend. Mit 1. Oktober 2012, der Eröffnung der neuen Fachbereichsbibliothek, konnte ausreichend Platz für die Bücher der beiden Fachrichtungen geschaffen werden.

Rund 110.000 Werke, davon auch Bestände aus der Hauptbibliothek sowie aus der Österreichischen Zentralbibliothek für Physik und Fachbereichsbibliothek Chemie, sind heute in der Fachbereichsbibliothek untergebracht. Ein Großteil dieser Bestände ist frei zugänglich. Die wertvollen Bestände sind in einem Magazin verwahrt und können in der Bibliothek benutzt werden.

Das Interesse an der neuen Fachbereichsbibliothek war von Anfang an sehr groß. Im ersten geöffneten Monat wurden ca. 6.500 Besucher_innen gezählt. Insgesamt 227 Personen nahmen an einer von 24 in den ersten zwei Monaten angebotenen Führungen durch die Bibliotheksräumlichkeiten teil.

Zahlreiche Rechercheschulungen, die direkt in den jeweiligen Lehrveranstaltungen abgehalten werden, fördern den Informationsfluss dort, wo er gebraucht wird. Die dafür nötige Zusammenarbeit mit Lehrveranstaltungsleiter_innen funktioniert wunderbar. Das Institut für Publizistik- und Kommunikationswissenschaft nimmt laut der aktuellen Ausgabe des Shanghai Ranking's Global Ranking of Academic Subjects 2018 für das Fach »Communication « nun den, weltweit gesehen, hervorragenden 13. Platz ein. ${ }^{2}$ Die Fach-

2 http://www.shanghairanking.com/Shanghairanking-Subject-Rankings/communication.html [Letzter Zugriff: 21.08.2019]. 
bereichsbibliothek ist stolz, sowohl dieses hochstrebende Institut mit mehr als 4.500 Studierenden als auch die innovative, schnell wachsende Fakultät für Informatik mit derzeit rund 2.400 Studierenden auf ihren erfolgreichen Wegen unterstützend zu begleiten. Die Zusammenarbeit mit zwei solch verschiedenen Fachbereichen gestaltet sich dabei für die Fachbereichsbibliothek zeitweise durchaus als herausfordernd, aber auch als spannend und abwechslungsreich. Interessanterweise zeigen sich viele Professor_innen der Fakultät für Informatik bei ihren Ankaufswünschen »bibliophiler« (»bitte die Print-Ausgabe») als ihre Kolleg_innen vom Institut für Publizistik- und Kommunikationswissenschaft (»bitte die Online-Ausgabe«).

Neben dem bibliothekarischen Tagesgeschäft hat sich die Fachbereichsbibliothek bereits dreimal als Austragungsort der beliebten »Nachtschicht@UB» zur Verfügung gestellt. Darüber hinaus unterstützt die Fachbereichsbibliothek freiwillig das OLIve-Projekt ${ }^{3}$, das Flüchtlingen einen niederschwelligen Zugang ermöglichen soll. Auch war die Fachbereichsbibliothek neben zahlreichen Fotoshootings Drehort eines Werbespots und einer Sequenz in Folge 5 der Krimiserie Der Pass. ${ }^{4}$

Die nächsten Jahre werden weiter von den Herausforderungen, die sich durch die soziale Interaktion mit den Besucher_innen und der Gestaltung der Fachbereichsbibliothek als öffentlichen Raum, stellen, geprägt sein. Die Beliebtheit des Standortes direkt an der sehr frequentierten Währingerstraße wird durch die voraussichtlich 2025 fertiggestellte U5-Station »Arne-Carlson-Park « wohl noch mehr zunehmen. Ende des Jahres 2018 wurde am Infopult das Abspielen von klassischem Musikprogramm eingerichtet, was für eine angenehme und konstruktive Atmosphäre sowohl für das Bibliotheksteam als auch für die Benutzer_innen bei ihrem Ankommen in der Bibliothek sorgen soll. Auf Nachhaltigkeit wird in dieser Fachbereichsbibliothek bereits seit Jahren großer Wert gelegt, weitere Nachhaltigkeitsmaßnahmen sollen umgesetzt werden. Natürlich stellen auch der kontinuierliche Ausbau des physischen und des elektronischen Bestandes sowie der Serviceangebote Aufgaben dar, die die Bibliothek, wie bisher, in sehr guter Zusammenarbeit mit den beiden Fachbereichen, deren Angehörigen und der Direktion meistern wird!

3 https://olive.univie.ac.at/home/olive-team/ [Letzter Zugriff: 21.08.2019].

4 http://www.epofilm.com/de/movie/der-pass/ [Letzter Zugriff: 21.08.2019]. 
Open-Access-Publikation im Sinne der CC-Lizenz BY-NC-ND 4.0

(c) 2019, Vandenhoeck \& Ruprecht GmbH \& Co. KG, Göttingen ISBN Print: 9783847110989 - ISBN E-Lib: 9783737010986 


\section{Bibliotheca semper reformanda. Oder warum sich an der Fachbereichsbibliothek Rechtswissenschaften so viel verändert hat}

\section{Allgemeines}

Die Fachbereichsbibliothek Rechtswissenschaften befindet sich seit 1984 im Juridicum in der Schottenbastei unweit vom Hauptgebäude der Universität. Einige Institute befinden sich in Gebäuden in der Schenkenstraße, weshalb auch ein Teil der rechtswissenschaftlichen Bücher in den Magazinsräumen der dortigen Fachbereichsbibliothek Theologie untergebracht ist.

Blickt man auf die letzten 15 Jahre zurück, so muss festgehalten werden, dass viele Veränderungen stattgefunden haben. Die Büroeinrichtungen wurden ebenso wie die fünf Informationsschalter und die Buchausgabe in der Lehrbuchsammlung komplett erneuert. Im Kellermagazin wurde eine Kompaktusanlage errichtet. Dadurch ergab sich die Möglichkeit, selten benötigte Zeitschriftenbände aus den Freihandbereichen ins Magazin zu verlagern und so Platz für laufende Zeitschriften zu schaffen. Seit 2018 verfügt die Fachbereichsbibliothek Rechtswissenschaften außerdem über einen Buchscanner. Für das Jahr 2019 ist die Errichtung einer Buchsicherungsanlage geplant, um so des doch beträchtlichen Bücherschwunds Herr zu werden.

Um wenigstens einen Teil der Investitionen mitzufinanzieren, findet an der Fachbereichsbibliothek laufend der Verkauf von ausgeschiedenen Büchern, vor allem aus dem Bereich der Lehrbuchsammlung, statt.

\section{Personalia}

Besonders umfangreiche Veränderungen erfolgten im Personalstand. Viele fixe Arbeitsplätze wurden geteilt und in geringfügige studentische Arbeitsplätze umgewandelt. Im Ergebnis umfasst die Fachbereichsbibliothek heute 18 Personen, die dem Stammpersonal zuzurechnen sind, und 14 studentische Mitarbeiterinnen und Mitarbeiter. Diese doch sehr hohe Anzahl ergibt sich durch den Umstand, dass die Bibliothek im Juridicum über keinen zentralen Eingang 
verfügt, sondern bei Vollbetrieb sechs unterschiedliche Stockwerke personell besetzt werden müssen.

Bemerkenswert ist auch, dass an der Fachbereichsbibliothek noch vier Beamte, gleichzeitig aber auch fünf ehemalige Lehrlinge tätig sind (Stand: Jänner 2019). Dies belegt die hohe Personalfluktuation und bedingt mit den ebenfalls oft wechselnden »Studis« einen hohen Bedarf an Schulungsmaßnahmen, die aber meist in Eigenregie durchgeführt werden können. Grundsätzlich wird der gesamte Geschäftsgang an der Fachbereichsbibliothek selbständig durchgeführt.

Im Betrachtungszeitraum musste die Fachbereichsbibliothek auch zwei fixe »B-Stellen" abgeben. Basis dafür war eine Evaluierung, die im Auftrag des Rektorats an der ganzen Universitätsbibliothek durchgeführt wurde und zum Ergebnis führte, dass eine Stelle im Bereich der Erwerbung und eine im Bereich der Katalogisierung zu viel vorhanden war. Durch eine Versetzung und eine Pensionierung wurde dem Einsparungswunsch entsprochen.

Diese beiden fehlenden B-Stellen brachten freilich Probleme bei der Einteilung der Infodienste und der Abwicklung des Geschäftsgangs mit sich. Auf Wunsch des Leiters der Fachbereichsbibliothek fand daher ein extern begleiteter Change-Management-Prozess statt, der allen Mitarbeiterinnen und Mitarbeitern der Fachbereichsbibliothek die Gelegenheit gab, sich aktiv an der Neugestaltung der Arbeitsvorgänge zu beteiligen.

Die Ausbildung der nächsten Generation von Bibliothekarinnen und Bibliothekaren ist der Fachbereichsbibliothek ein großes Anliegen. So ist nicht nur der Leiter der Fachbereichsbibliothek in den verschiedenen Ausbildungszweigen aktiv, sondern die ganze Fachbereichsbibliothek leistet seit Jahren einen großen Beitrag bei der Betreuung des Nachwuchses im Rahmen von Praktika.

\section{Benutzung \& Kommunikation}

Sowohl im Rahmen der regelmäßigen Evaluierungen der gesamten Universitätsbibliothek, als auch im Zusammenhang mit dem oben erwähnten ChangeManagement-Prozess fanden Benutzerbefragungen unter den »Powerusern « der Fachbereichsbibliothek statt. Diese Umfragen führten neben unerfüllbaren Wünschen (v.a. mehr Leseplätze) zu konkreten Maßnahmen: So wurden die Öffnungszeiten während des Semesters dahingehend ausgeweitet, dass ein Freihandbereich bis 21:45 Uhr statt bis 19:00 Uhr geöffnet bleibt. Ebenso wird an den zwei Samstagen, die jeweils vor einer Prüfungswoche liegen, ein Freihandbereich von 9:00 bis 17:00 Uhr geöffnet, um den Studierenden mehr Platz zum Lernen anzubieten.

Besonders wurde bei einer Evaluierung kritisiert, dass Plätze reserviert und danach stundenlang nicht genutzt werden. In einer längeren Diskussion mit der 
Fakultätsvertretung und Studierenden wurde dann das System der »Pausenscheine« implementiert. Diese gestatten zwar eine Abwesenheit, erlauben aber anderen Benutzerinnen und Benutzern, diesen Platz während der Absenz zu verwenden. Dadurch konnte die Anzahl der Benutzungsvorgänge um etwa 10.000 pro Jahr gesteigert werden.

Überhaupt ist die Kommunikation mit den Studierenden ein sehr großes Anliegen der Fachbereichsbibliothek. Wichtige Nachrichten werden daher nicht nur auf der eigenen Website und mittels Aushängen, sondern auch über den Newsletter und den Facebook-Account der Fakultätsvertretung verbreitet. Außerdem findet sich in jeder Ausgabe des »Juristl«, der Zeitschrift der Fakultätsvertretung Jus, ein Beitrag, der über Neuigkeiten aus der Bibliothek berichtet. Diese gute Zusammenarbeit zeigt sich auch darin, dass seitens der Fakultätsvertretung immer wieder Geldmittel zur Verbesserung von bibliothekarischen Services zur Verfügung gestellt werden. So konnten viele Bücher für die Lehrbuchsammlung zusätzlich erworben und eine Buchrückgabebox angeschafft werden.

Ein weiteres Anliegen, das bei den Evaluierungen immer wieder geäußert wurde, ist der Wunsch, dass der gesamte Bestand der Fachbereichsbibliothek im Online-Katalog verzeichnet sein sollte. Dies gestaltet sich an der Fachbereichsbibliothek leider schwierig, da die Bestände im Jahr 1984 aus den drei damals an der Fakultät bestehenden Seminarbibliotheken zusammengesetzt wurden, die oft von ungeschultem Personal betreut worden waren. Es ist daher notwendig, alle Bücher aus den Regalen zu holen, um so korrekte Katalogisate gewährleisten zu können.

Nach einer Änderung des Curriculums für Rechtswissenschaften, die zwei Seminare für alle Studierenden zur Pflicht machte, wurde beschlossen, die bisherige reine Präsenzbibliothek in eine Bibliothek mit Entlehnmöglichkeit umzustellen.

\section{Elektronische Ressourcen}

Wie in der gesamten Bibliothekswelt veränderte sich auch das Angebot an der Fachbereichsbibliothek Rechtswissenschaften hin zu mehr elektronischen Ressourcen, wenngleich diese Entwicklung in anderen Wissenschaftsgebieten sicher stärker ausgeprägt ist. War zunächst die juristische österreichische Zeitschriftenliteratur in der RDB vereint zugänglich, so spalteten sich mit der Zeit die anderen juristischen Fachverlage ab und machten sich selbstständig. Dieser Zuwachs an Datenbanken fand naturgemäß auch in den Kosten einen starken Niederschlag, da seitdem jeder Verlag zu bezahlen ist. Diese erhöhten Kosten gingen zu Lasten der Medien, die in Print angeschafft werden konnten, was 
immer wieder zu Konflikten mit der Fakultät führte, die letztendlich durch eine Erhöhung der Finanzmittel beigelegt werden konnten.

Im Vergleich $\mathrm{zu}$ anderen juristischen Bibliotheken in Österreich ist die Fachbereichsbibliothek Rechtswissenschaften dennoch nur mittelmäßig aufgestellt, was vor allem daran liegt, dass die Kosten für elektronische Medien zumeist auf Basis der Anzahl der Studierenden seitens der Verlage berechnet werden. Da die Fachbereichsbibliothek Rechtswissenschaften der Universität Wien doppelt so viele Studierende hat wie jene der Universität Graz, die an zweiter Stelle liegt, fallen bei elektronischen Ressourcen in Wien deutlich höhere Kosten an.

Die vielen elektronischen Ressourcen verlangten natürlich auch ein verstärktes Schulungsangebot, welches seitens der Fachbereichsbibliothek regelmäßig angeboten wird. Im Jahr 2018 ergab sich darüber hinaus für die Fachbereichsbibliothek die Gelegenheit, die Nutzung der Datenbanken im Rahmen einer Lehrveranstaltung zu vermitteln, die für Studierende im ersten Abschnitt verpflichtend ist. Dafür wurde zu jeder Datenbank ein Video aufgenommen, welches von den Studierenden, aber auch von allen anderen Interessierten auf der Website angesehen werden kann.

Für die kommenden Jahre stehen sicher der Ausbau der elektronischen Ressourcen und die stete Verbesserung der Services auf der Agenda der Fachbereichsbibliothek. Was dabei alles zu tun sein wird, kann heute nur erahnt werden. Spannend bleibt es mit Sicherheit! 


\section{Bibliothécaire/bibliotecaria/bibliotecária/bibliotecară. Bibliothekarinnen an der Fachbereichsbibliothek Romanistik}

Die Universitätsbibliothek Wien als größte Universitätsbibliothek Österreichs wird bereits seit mehr als einem Vierteljahrhundert von Frauen geleitet. Seit 2004 steht Maria Seissl an der Spitze der Bibliothek, ihre Vorgängerin Ilse Dosoudil führte diese von 1993 bis 2003.

Gegen Ende des 19. Jahrhunderts durfte eine junge Frau einzelne Vorlesungen an der Universität Wien als Gasthörerin besuchen, und es war ihr im Zuge dessen vermutlich auch erlaubt, die 1870 als Seminarbibliothek für französische und englische Sprache gegründete Bibliothek zu benutzen. In die Geschichte ging sie nicht nur als erste Frau ein, die mit 31 Jahren als Externistin am Akademischen Gymnasium in Wien maturierte, sondern Elise Richter (1865-1943) war auch die erste, die sich 1905 an der Universität Wien habilitieren konnte und ebenfalls als erste Frau 1921 zum außerordentlichen Professor (sic!) an der Wiener Romanistik ernannt wurde.

Einige ihrer Privatbücher befinden sich bis heute unrechtmäßig in der Fachbereichsbibliothek Romanistik. Diese Bücher wurden von der NS-Provenienzforschung der Universitätsbibliothek Wien als zu restituierender Bestand eingestuft, die Erbensuche läuft noch.

Nicht nur dieser Bestand erinnert heutige Bibliothekar_innen und Studierende an die Geschichte - auch die vielen NS-Stempel, die in allen Büchern zu finden sind, die vor 1945 an die Bibliothek kamen. Denn in den Jahren 1938 bis 1945 hatte Universitätsprofessor Joseph Huber (1884-1960) die Verwaltung der Romanistik-Bibliothek inne, und unter seiner Ägide wurde der gesamte Buchbestand umsigniert und mit dem Rundstempel der NS-Verwaltung versehen. Noch heute sind immer wieder Benutzer_innen verwundert und teilweise irritiert, dass sich diese historischen Zeichen immer noch in den Büchern befinden. 


\section{Romanistik als Frauenstudium}

Von Elise Richter bis heute hat sich das Studium der Romanistik in großem Maße zu einem Frauenstudium entwickelt. In den letzten 15 Jahren liegt die Zahl der weiblichen Studierenden konstant bei 83-87 \%. Auch die Fachbereichsbibliothek Romanistik wird seit 30 Jahren stark von Frauen geprägt. Seit die Institutsbibliothek für Romanistik im Jahre 1986 zur Fachbibliothek und später Fachbereichsbibliothek Romanistik wurde, übernahm die Leitung der Bibliothek erstmals eine Bibliothekarin. Maria Lauber führte die Bibliothek bis zu ihrer Pensionierung im Dezember 2007 nahezu zwei Jahrzehnte lang. Eine von ihr 1998 entworfene Aufstellungssystematik, die im Großen und Ganzen nach Sprach- und Literaturwissenschaft der einzelnen romanischen Sprachen sowie nach Landes- und Kulturwissenschaft einteilt, ist noch heute in Verwendung. Im Studienjahr 2008/09 wurde ein mit dem Institut für Romanistik ausgearbeiteter Vorschlag zur Modernisierung der Aufstellung der linguistischen Bestände erarbeitet, konnte aber leider aus finanziellen Gründen nicht verwirklicht werden. Nach einigen Jahren, in denen zumindest je ein studentischer Mitarbeiter in der Fachbereichsbibliothek tätig war, ist das aktuelle Team mit sieben Mitarbeiterinnen wieder zu 100 Prozent weiblich.

\section{Frauen an der Universitätsbibliothek Wien}

Die generelle Entwicklung des Berufbildes »Bibliothekar_in« innerhalb des österreichischen Bibliothekswesens und damit auch an der Universitätsbibliothek Wien hin zu einem Frauenberuf nahm ihren Anfang in den 1970er Jahren. Neben einigen gesetzlichen Veränderungen, die die Stellung der Frau in der Gesellschaft und damit auch im Berufsleben verbesserten, kam den Frauen in den wissenschaftlichen Bibliotheken vor allem die Förderung durch Hertha Firnberg (1909-1994) als Ministerin für Wissenschaft und Forschung, aber auch durch Johanna Dohnal (1939-2010) als Staatssekretärin für allgemeine Frauenfragen zugute. Ab 1981 war mit Ministerialrätin Edith Stumpf-Fischer eine Frau als Abteilungsleiterin des wissenschaftlichen Bibliothekwesens an zentraler Stelle tätig. Sie bemühte sich sehr um qualifizierte Bibliothekarinnen und förderte Frauen, wo immer es möglich war.

Im Jahr 1977 waren an der Universitätsbibliothek Wien von 173 Bediensteten 47 Frauen, überwiegend im Reinigungs- und Magazinsdienst, Maturantinnen in der Formalerschließung und Erwerbung tätig. Unter den 38 Akademiker_innen gab es gerade einmal fünf Frauen - keine der Bibliothekarinnen war jedoch in leitender Position tätig. Erst in der zweiten Hälfte der 1980er Jahre begann sich die Lage an der Universitätsbibliothek Wien grundlegend zu ändern. Im Jahr 
1990 waren von 20,5 A-Bediensteten an der Hauptbibliothek bereits elf Frauen. Darunter Ilse Dosoudil als einzige in einer leitenden Position. Sie war zu diesem Zeitpunkt nicht nur Vizedirektorin und zuständig für den dezentralen Bereich, sondern auch Leiterin der Ausbildungsabteilung.

In den Fach- und Fakultätsbibliotheken der Universitätsbibliothek Wien hatte sich die Lage in Folge des Universitätsorganisationsgesetzes 1975 und durch die vielen Neubesetzungen bereits geändert. Hier wurden Anfang der 1990er Jahre immerhin neun von 28 Teilbibliotheken von einer Akademikerin geführt. Es waren dies: Renate Niegl (verh. Klepp, 1955-2013), die die Fakultätsbibliothek für Rechtswissenschaften sowie die Informationsvermittlungstelle der Rechtswissenschaften leitete, sowie die Fachbibliotheksleiterinnen Leopoldine Swoboda (Erziehungswissenschaften), Gertraut Schikola (Kunstgeschichte), Judit Ramhardter (Ost- und Südeuropaforschung), Helga Trunk (Philosophie), Michaela Zemanek (Psychologie), Maria Aldouri-Lauber (Romanistik), Helga Kerchler (Ur- und Frühgeschichte) und Erika Neuber (Völkerkunde). Im Frühjahr 2019 ging mit Michaela Zemanek die letzte dieser Pionierinnen in den Ruhestand.

Mit der Ernennung von Magda Strebl zur Generaldirektorin der Österreichischen Nationalbibliothek im Jahr 1984 wurde österreichweit eine gläserne Decke durchbrochen. Heute werden gerade die Universitätsbibliotheken zu gut der Hälfte von Frauen geleitet. Seit den 1990er Jahren werden jedenfalls auch die größten Bibliotheken des Landes von Frauen geführt. Seit 2001 steht Johanna Rachinger an der Spitze der Österreichischen Nationalbibliothek. Die Universitätsbibliothek Wien wird, wie bereits erwähnt, seit 1993 von Frauen geleitet. An der Universitätsbibliothek Wien gibt es heute laut Personalverzeichnis ein Geschlechterverhältnis von ca. 3:2 zugunsten von Frauen. Von 37 Fachbereichsbibliotheken werden 24 von einer Bibliothekarin geführt, d. h. auch hier ein über 60\%iger Frauenanteil und in Summe bibliotheksweit eine 50:50 Quote in den Leitungsfunktionen, was bedeutet, dass bei einer Stellenbesetzung wirklich die bestqualifizierte Person genommen werden kann, ohne auf eine gewisse Quote achten zu müssen.

Auch die Frage nach Kind oder Karriere scheint heute eine subjektive Entscheidung zu sein. Wenngleich die Vereinbarkeit von Familie und Beruf nach wie vor häufig ein Drahtseilakt ist, so finden die Mitarbeiterinnen und Mitarbeiter an der Universitätsbibliothek Wien unter der Leitung von Maria Seissl volle Unterstützung auch in schwierigen privaten Situationen und immer ein offenes Ohr. Die Möglichkeit, mit einer Teilzeitanstellung eine Fachbereichsbibliothek leiten zu können - wie aktuell auch an der Fachbereichsbibliothek Romanistik - ermöglicht es vielen Kolleg_innen, auch nach einer Karenz ihre Arbeit wieder an gleicher Position fortführen zu können. 
Auch an anderen wissenschaftlichen Bibliotheken in Österreich gibt es ein relativ ausgeglichenes Verhältnis von Männern und Frauen in Führungspositionen. Es zeigt sich, dass die verstärkte Frauenförderung seit den 1980er Jahren im Bibliothekswesen Früchte getragen hat. Das ist eine erfreuliche Entwicklung angesichts der Tatsache, dass der Bibliothekarsberuf jahrhundertelang ein reiner Männerberuf war. 


\section{»Ich mag meine Fachbereichsbibliothek «. Empathie, Diskurs und Forschungsinteresse an der Fachbereichsbibliothek Soziologie und Politikwissenschaft}

Umsichtig und engagiert leitet Maria Seissl seit 2004 die Universitätsbibliothek Wien. Kurz zuvor war sie in ihrer Funktion als Vizedirektorin bereits maßgeblich an der gelungenen Zusammenführung mehrerer Institutsbibliotheken zur heutigen Fachbereichsbibliothek Soziologie und Politikwissenschaft beteiligt: die Bibliotheken der Institute für Soziologie an der Sozial- und Wirtschaftswissenschaftlichen Fakultät und an der Grund- und Integrativwissenschaftlichen Fakultät sowie die Bibliothek des Institutes für Politikwissenschaft bilden seit dem Jahr 2002 die Basis für den heute vielfältigen Bestand der Fachbereichsbibliothek Soziologie und Politikwissenschaft, kurz »FB Sozpol«, im Haus der Soziologie am Rooseveltplatz 2.

Die Bibliothek betreut ihren zweiten Standort am Institut für Wissenschaftsund Technikforschung im Neuen Institutsgebäude und konnte in den vergangenen Jahren weitere Bestände in den Hauptstandort am Rooseveltplatz integrieren: So stellt die Bibliothek seit 2005 Literatur zum jungen Forschungsgegenstand der Pflegewissenschaft zur Verfügung. Im Sommer 2012 wurde die Bibliothek des Institutes für Staatswissenschaft eingegliedert, im gleichen Jahr eine großzügige Schenkung aus dem Nachlass von Heinz Steinert übernommen sowie 2016 eine lohnende Erweiterung mit der Bibliothek des European Centre for Social Welfare Policy and Research durchgeführt. Zuletzt wurde eine Auswahl der Bibliothek des Wiener Instituts für sozialwissenschaftliche Dokumentation und Methodik (WISDOM) erfasst, welches als Vorläufer von AUSSDA, dem "Austrian Social Science Data Archive«, zu sehen ist. Parallel dazu wird seit Jänner 2019 die pflegewissenschaftliche Abteilung durch eine Übernahme der Fachbibliothek Palliative Care und Organisationsethik bereichert.

Unsere Fachbereichsbibliothek konnte zudem im Jahr 2003 die Verwaltung des 1983 eröffneten Paul F. Lazarsfeld-Archivs übernehmen: Als Gedächtnis der Sozialforschung in/aus Österreich beinhaltet das Archiv die wissenschaftlichen Nachlässe des 1976 verstorbenen Soziologen Paul Felix Lazarsfeld, weithin bekannt durch die frühe empirische Studie Die Arbeitslosen von Marienthal, sowie des Soziologen und Statistikers Paul Martin Neurath (1911-2001), gleichzeitig 
einer der Begründer des Archivs. Geleitet wird das Archiv vom Soziologen und Gerontologen Anton Amann, welcher gemeinsam mit Paul Neurath das Archiv aufgebaut hat. Das Paul F. Lazarsfeld-Archiv umfasst sämtliche Publikationen (Bücher, Aufsätze, Zeitschriften- und Zeitungsartikel, etc.) sowie unpublizierte Arbeiten und Teile der wissenschaftlichen Korrespondenz, zahlreiche Forschungsberichte, Arbeitsunterlagen sowie Dokumente der beiden Sozialforscher. Zu den Beständen des Archives zählen zudem deren Arbeitsbibliotheken, die Erschließung dieser für die historische Sozialforschung wertvollen Buchbestände wurde kürzlich abgeschlossen. Im aktuellen Sommersemester 2019 wird im Rahmen eines Ausbildungsprojektes des Universitätslehrganges Library and Information Studies an der Verbesserung der (Metadaten-)Struktur, der Sichtbarkeit des Archives und der Nutzbarkeit der Archivalien gearbeitet.

Die vielfältige Zusammensetzung der Bestände der Fachbereichsbibliothek steht dem optischen ersten Eindruck der Benutzer_innen entgegen: Die Bibliothek wirkt kompakt, nicht wirklich weitläufig. Immerhin aber kann sie auf einen physischen Bestand von etwa 120.000 Bänden verweisen, ein großer Teil der Bücher wird im verwinkelten Kellermagazin bewahrt und kann online zur Benutzung bestellt werden.

Eine Bibliothek im Altbaukeller bedarf einer Schar wassereinbruchsgeeichter Bibliothekar_innen. Kaum zu glauben, wie erfolgreich hier Hunderte Laufmeter Buch vor Wassereinbrüchen gerettet werden können, Wasser kann von unten, von oben, seitlich, aus Haustechnikanlagen austreten oder ausströmen, allerdings ist ob engagierter Rettungsaktionen noch kein einziges Buch diesen feuchten Angriffen zum Opfer gefallen!

Oberirdisch gibt sich die Bibliothek in ihren attraktiven Räumlichkeiten mit Blick auf den Votivpark einladend. Hier stehen die Bestände in Kompaktregalen im Freihandbereich zur Verfügung. Trotz der räumlichen Überschaubarkeit oder gerade deswegen ist die Fachbereichsbibliothek Soziologie und Politikwissenschaft ein äußerst beliebter Lernort. Mitunter könnte man meinen, die Besucher_innen würden sich in den Lesesälen stapeln wollen, könnten sie es. Wir versuchen laufend, die Infrastruktur zu verbessern und den sich verändernden Bedürfnissen anzupassen: So konnte zuletzt ein Lautarbeits- und Besprechungsraum adaptiert werden, hier wurde auch ein beliebtes Zuckerl für die Benutzer_innen aufgestellt: der Buchscanner läuft im Dauerbetrieb.

Unsere Benutzer_innen sind uns seit jeher sehr wichtig: An der Fachbereichsbibliothek besteht ein großes Bemühen, den Spagat zwischen niederschwelliger Offenheit und einem breitgefächerten Angebot an kompetenter Rechercheunterstützung und einer hohen Qualität des Bestandes zu schaffen. Zentrale Aufgabe ist es, Literatur für unser breites Spektrum an Forschungsbereichen und Lehrinhalten bereitzustellen und Wissenschafter_innen und Studierende an das umfangreiche (Service-)Angebot der Universitätsbibliothek 
heranzuführen sowie die nötigen Nutzungsmodalitäten für Forschungsinfrastruktur und -inhalte zu vermitteln. Veränderte Publikationsstrategien und -modelle in den Sozialwissenschaften sowie hohe Studierendenzahlen verlangen nach den entsprechenden Mitteln: der sukzessive Ausbau des Angebots an elektronischen Ressourcen und ein Paradigmenwechsel zugunsten vielfältiger Erscheinungsformen und Aktualität des Medienangebots kommt diesen Herausforderungen entgegen.

Nichtsdestotrotz bleibt die Bibliothek als physischer Raum unabdingbar: als Ort des Lesens, des Lernens und der Begegnung, sowie als Ort des gesellschaftlichen Diskurses:

Die Fachbereichsbibliothek fungierte in den vergangenen Monaten als begehrter Ausstellungs- und Veranstaltungsort. Die Ausstellung Zuflucht von Nina Zuckerstätter zeigte im Sommersemester 2018 Bilder der humanitären Krise auf der griechischen Insel Lesbos.

Anlässlich des Gedenkjahres 1938/2018 wurden Buchobjekte des bildenden Künstlers Hannes Priesch ausgestellt: die Fachbereichsbibliothek veranstaltete in Zusammenarbeit mit den Instituten für Politikwissenschaft, Staatswissenschaft und Zeitgeschichte das Art \& Science-Projekt »1000-jährige Bibliothek die Sprache prüfen«. Der Fokus lag auf der künstlerischen und wissenschaftlichen Auseinandersetzung mit radikaler politischer Sprache in Vergangenheit und Gegenwart, ausgehend von Adolf Hitlers Mein Kampf. Das Begleitprogramm zur Ausstellung mit Beiträgen von u. a. Petra Bernhardt, Ingrid Brodnig, Rainer Fuchs, Peter Huemer, Walter Manoschek, Roman Pfefferle, Oliver Rathkolb, Birgit Sauer, Johanna Schwanberg, Katharina Stemberger und Ruth Wodak bot einen anregenden Einblick in historische und zeitgenössische politische Debatten und forderte zur kritischen Prüfung von Sprache und politischen Prozessen auf. Den Besucher_innen wurde ergänzend zur Buchinstallation von Hannes Priesch ein ausgesuchter Literaturapparat zum Thema »Politik und Sprache« bereitgestellt.

Im Frühjahr 2019 wurde die Bibliothek im Rahmen der »@ FOTO Wien« zur Präsentationsfläche für »Rough Sleep«, einer künstlerischen Fotodokumentation zur Kritik am Quantified Self: "Nullstellung«, so der Untertitel, ist die Haltung, welche die Künstlerinnen Katharina Brandl, Violeta Ivanova, Madeleine Schrabauer und Karla Woess in ihrer widerständigen Praxis im öffentlichen Raum anhand von Schlafperformances zeigt.

Schlafen würden vermutlich nicht selten die einen oder anderen Benutzer_innen nächtens bevorzugt gleich in der Bibliothek, viele halten sich gerne hier auf und fühlen sich gut betreut. Die Mitarbeiter_innen der Fachbereichsbibliothek bilden ein Team, das Wertschätzung untereinander sowie gegenüber den Besuchenden als wesentlich ansieht. Diese Maxime wurde von der vormaligen Leiterin, Eva Sibitz, gelebt und wird weiterhin fortgeführt. Wir freuen uns 
zudem, wenn wir Praktikant_innen oder Lehrlinge im Team begrüßen und sie ein Stück weit auf ihrem bibliothekarischen Ausbildungsweg begleiten können.

Feedback seitens unserer Benutzer_innen erhalten wir durchwegs positives: Bewertungen wie »beeindruckendes Engagement und Kompetenz« oder »bestes Team ever « freuen uns sehr. Dadurch motiviert werden wir in den kommenden Jahren unter der Leitung von Maria Seissl mit ihren Vorstellungen von der wissenschaftlichen Bibliothek im Sinne von Offenheit und Zugänglichkeit nicht nur an Ressourcen wachsen, sondern auch an Kompetenzen für Vermittlung, Forschungsunterstützung und die Bereitstellung von Wissen sowie an Serviceorientiertheit, auf dass die Fachbereichsbibliothek Soziologie und Politikwissenschaft weiterhin mit »Ich mag meine Fachbereichsbibliothek" evaluiert werden möge. 


\section{Eine Transformation. Von der Institutsbibliothek zur Fachbereichsbibliothek Sportwissenschaft}

Das Zentrum für Sportwissenschaft und Universitätssport (ZSU) wurde 1973 fertiggestellt ${ }^{1}$ und hatte eine über Jahrzehnte gewachsene, eigenständige Organisationsstruktur innerhalb der Universität Wien, die eng mit dem damaligen Bundesministerium für Unterricht und Kunst verflochten war. Dem ZSU sind das Institut für Sportwissenschaft (ISW) und das Universitätssportinstitut (USI) der Universität Wien zugeordnet. ${ }^{2}$ Ebenso sind die Bundesanstalt für Leibeserziehung (heute: Bundessportakademie, BSPA, die dem Bundesministerium für Bildung, Wissenschaft und Forschung, BMBWF, zugeordnet ist) ${ }^{3}$, das Universitätssportzentrum (heute: Zentrale für Sportgeräteverleih und Sportplatzwartung, ZSSW, dem BMBWF zugeordnet $)^{4}$ und das Österreichische Institut für Sportmedizin (ÖISM, das als Stiftung organisiert ist, der Vorsitz wird durch eine/n Vertreter/in der Universität Wien geführt) am selben Standort auf der Schmelz verortet.

Für die Bibliothek auf der Schmelz bedeutete dies über viele Jahre hinweg eine Verflechtung der Zuständigkeiten, was sowohl budgetär als auch personell eine große Rolle spielte. Die finanziellen Mittel für den Ankauf von Büchern wurden von der Universität Wien zur Verfügung gestellt und jene für den Ankauf von Zeitschriften vom Bundesministerium für Bildung, Wissenschaft und Forschung. Entsprechend dazu wurde auch das Personal von diesen beiden Stellen gestellt. Die Leitung der Institutsbibliothek und zwei Mitarbeiter_innen gehörten dem Institut für Sportwissenschaften an, weitere dreieinhalb vollzeitäquivalente Stellen unterstanden dem Bildungsministerium. Dies führte über die Jahre oft zu Zuständigkeitsproblemen. Die Bibliothek war bereits sehr früh, nämlich schon zu Zeiten des frühen Bibliothekssystems BIBOS in den 1980er Jahren, im Gesamtbibliothekskatalog der Universitätsbibliothek Wien vertreten.

1 Hannes Strohmeyer: Beiträge zur Geschichte des Sports in Österreich. Gesammelte Arbeiten aus vier Jahrzehnten. Wien: öbv \& hpt 1999, S. 403.

2 https://zsu-schmelz.univie.ac.at/ueber-uns/[Letzter Zugriff: 23.01.2019].

3 http://www.bspa.at/organisation/ueber-uns/ [Letzter Zugriff: 23.01.2019].

4 https://www.schulsport-serviceteam.at/ [Letzter Zugriff: 23.01.2019]. 
Für den damaligen Bibliotheksleiter bedeuteten die unterschiedlichen Anforderungen der beiden Träger des Zentrums einen nicht zu unterschätzenden bürokratischen Mehraufwand. Um das Jahr 2010 wurde immer offensichtlicher, dass diese Verflechtung auf Dauer nicht aufrechtzuerhalten war. Wie so oft war die Frage des Geldes zentral und letztendlich bedeutend für die gesamte Einrichtung und Organisation des Universitätssportzentrums auf der Schmelz. Das Bildungsministerium initiierte die Zusammenlegung des Sportgeräteverleihs mit der Abteilung für Sportplatzwartung und im Zuge dieser Zusammenlegung wurde auch die Bibliothek zum Thema. Dies führte zunächst zu einer zunehmenden Unsicherheit unter den Bibliotheksmitarbeiter_innen und in weiterer Folge $\mathrm{zu}$ vielen Gesprächen zwischen den einzelnen Parteien, um zu einer möglichst optimalen Lösung zu kommen.

Als Direktorin der Universitätsbibliothek Wien hatte Maria Seissl schon seit einiger Zeit signalisiert, dass sie einer Eingliederung der Institutsbibliothek, die tatsächlich aber als Zentrumsbibliothek geführt wurde, in die Dienstleistungseinrichtung (DLE) Bibliotheks- und Archivwesen der Universität Wien offen gegenüberstand. Nach einem relativ kurzen Verhandlungszeitraum von etwa einem halben Jahr wurde zwischen den Parteien eine entsprechende Übereinkunft getroffen.

Am 1. Jänner 2013 war es dann so weit: die Institutsbibliothek Sportwissenschaft wurde der Dienstleistungseinrichtung Bibliotheks- und Archivwesen angeschlossen und führt seither den Namen Fachbereichsbibliothek Sportwissenschaft. Durch die organisatorische Veränderung wechselte auch die Leitung der Bibliothek. Rudolf Müllner verblieb auf eigenen Wunsch am Institut für Sportwissenschaft, um seiner Tätigkeit in Wissenschaft und Lehre nachzukommen, und die Autorin dieses Beitrags, die bereits seit 2004 als Bibliothekarin an der Universitätsbibliothek Wien tätig war, übernahm die frei gewordene Position. Die beiden Bibliotheksmitarbeiterinnen, die bis dahin als Institutsmitarbeiterinnen tätig waren, wurden in den Mitarbeiterstab der DLE Bibliotheks- und Archivwesen aufgenommen. Die dreieinhalb Stellen, die der Bibliothek vom Bundesministerium zugeteilt waren, wurden in andere Abteilungen des Ministeriums übergeführt.

Durch die erhebliche Personalreduzierung war die Notwendigkeit gegeben, verschiedene Maßnahmen durchzuführen, um die Serviceleistungen für die Benutzer_innen aufrechtzuerhalten bzw. zu verbessern. Daher wurden zwei Büros, die für ihren ursprünglichen Zweck nicht mehr benötigt wurden, in einen Gruppenarbeitsraum bzw. in einen Kopier-, Druck- und Scan-Bereich umgewandelt. Weiters wurde die Garderobe räumlich verändert, um zusätzliche Leseplätze zu gewinnen und die Aufstellung der aktuellen Printzeitschriften zu optimieren. 
Eine weitere Maßnahme war die Implementierung der RFID-Technologie zur Buchsicherung und Verbuchung der Bestände im Bereich der Entlehnung. Die Einführung der RFID-Technik wurde mit der Absicht vorgenommen, die bis dahin bestehende Bestell-Bibliothek in eine Freihandaufstellung umzuwandeln, wodurch die ressourcenaufwändigen Aushebungen von Buchbestellungen wegfallen. Es wird damit gerechnet, dass die Implementierung in absehbarer Zeit vollständig umgesetzt werden kann. Als zusätzliches Service wurde ein Bücherrückgabekasten vor den Bibliotheksräumlichkeiten aufgestellt und ein Buchscanner von der Bibliothek angekauft.

Eine der großen Herausforderungen, welche den Großteil der wissenschaftlichen Bibliotheken Österreichs in den letzten zweieinhalb Jahren begleitet hat, war die Umstellung auf ein neues Bibliothekssystem und die nahezu zeitgleiche Einführung einer neuen Studierenden-Karte an der Universität Wien, die zugleich als Bibliotheksausweis fungiert. Dieser Prozess ist nach wie vor nicht abgeschossen, funktioniert aber mittlerweile weitgehend gut. Für die Fachbereichsbibliothek Sportwissenschaft war es nicht ganz einfach, die vielen dafür nötigen Schulungen zeitlich für alle Mitarbeiter_innen möglich zu machen und dabei die normalen Öffnungszeiten aufrechtzuerhalten. Durch die relativ große räumliche Trennung von den anderen Standorten der Universitätsbibliothek das Zentrum für Sportwissenschaft liegt etwas außerhalb auf der sogenannten "Schmelz«, einem ehemaligen Exerzier- und Paradeplatz im 15. Wiener Gemeindebezirk - musste allein schon für die Wegstrecke nicht wenig Zeit miteingeplant werden. Durch die gute Zusammenarbeit des Teams und die Unterstützung der Bibliotheksleitung wurde aber auch diese Aufgabe gemeistert.

Als größte sportwissenschaftliche Bibliothek Österreichs und nach der Bibliothek der Deutschen Sporthochschule Köln zweitgrößten im deutschen Sprachraum $^{5}$ stellt die Fachbereichsbibliothek Sportwissenschaft einen besonderen Referenzpunkt nicht nur für die Studierenden und wissenschaftlichen Mitarbeiter_innen des Instituts für Sportwissenschaft dar, sondern wird auch sehr gerne von Studierenden anderer Bildungseinrichtungen, der interessierten Öffentlichkeit und seit der verpflichtenden Einführung einer Vorwissenschaftlichen Arbeit im Rahmen der Reifeprüfung seit dem Schuljahr 2013/14 ${ }^{6}$ zunehmend von Schüler_innen besucht. Dieser vermehrte Andrang einer bis dahin nicht sehr großen Benutzer_innengruppe stellte die Bibliothek und ihre Mitarbeiter_innen vor neue Aufgaben, speziell als Teaching Library. Die notwendigen Schulungsmaßnahmen werden hauptsächlich im persönlichen Gespräch durchgeführt.

5 Rudolf Müllner: Perspektiven der historischen Sport- und Bewegungskulturforschung. Wien: Lit 2011, S. $102 \mathrm{ff}$.

6 http://www.veritas.at/about/presse/vorw_arbeit [Letzter Zugriff: 23.01.2019]. 
Aufgrund einer Veränderung im Curriculum des Lehramtsstudiums wurde die Möglichkeit von Schulungsmaßnahmen für Studierende, die bis zum Studienjahr 2017/2018 regelmäßig durchgeführt wurden, stark eingeschränkt. Durch die Zusammenarbeit der Pädagogischen Hochschulen mit den Universitäten wurden viele einführende Lehrveranstaltungen zugunsten von Unterricht in ebendiesen eingestellt und damit auch die darin vorgesehenen Bibliotheksschulungen im Bereich der Sportwissenschaft. Als mögliche Kompensation ist eine fachspezifisch angepasste elektronische Schulung über die Lernplattform der Universität Wien angedacht.

In ihrem Blick in die Zukunft unterscheidet sich die Fachbereichsbibliothek Sportwissenschaft nicht von anderen wissenschaftlichen Bibliotheken: Die räumlichen Herausforderungen, denen sich die Universität Wien derzeit und in naher Zukunft gegenübersieht, werden auch vor den anderen Standorten der Universitätsbibliothek Wien nicht Halt machen und Neuorganisationen mit sich bringen. Nicht zuletzt erfordert das digitale Zeitalter und die damit einhergehenden Veränderungen im Verhalten der Bibliotheksnutzer_innen, sowie die sich immer schneller entwickelnden technischen Neuerungen und der Umgang mit dem ständig wachsenden Bestand an elektronischen Ressourcen, eine flexible und offene Haltung. 


\section{Desperately seeking library relocation. Ein neuer Standort für die Fachbereichsbibliothek Theater-, Film- und Medienwissenschaft}

Mit der Übersiedlung der Fachbereichsbibliothek Theater-, Film- und Medienwissenschaft im April 2017 in das Universitätszentrum Althanstraße der Universität Wien fand ein langwieriges Projekt ein überaus glückliches Ende.

Pläne, die Fachbereichsbibliothek aus Platzgründen von ihrem Standort in der Wiener Hofburg abzusiedeln, gab es im Laufe der Jahre seit ihrem Bestehen immer wieder. Mit der Umwidmung des Komplexes des früheren Allgemeinen Krankenhauses der Stadt Wien zum Campus der Universität Wien hätte eine Übersiedlung der Bibliothek Ende der 1990er Jahre konkretisiert werden können. Damals sprach sich das Institut jedoch dagegen aus. Fünfzehn Jahre später, im Jahr 2013, wurde die Verlegung des Standortes dann definitiv beschlossen. Diese Entscheidung kam für die Fachbereichsbibliothek gerade zum richtigen Zeitpunkt. Verschiedene Umbauarbeiten in den Institutsräumlichkeiten in der Hofburg von Seiten der Burghauptmannschaft führten zu einer drastischen Verkleinerung der Bibliotheksnutzfläche, wodurch viel Stellplatz verloren ging und wovon vor allem auch Bereiche für die Benutzer_innen betroffen waren. Die Wahl für den zukünftigen Standort fiel letztlich auf den Gebäudeteil Rotunde des Universitätszentrums Althanstraße II (UZA II), welcher im Zuge der Absiedlung der Wirtschaftsuniversität Wien frei wurde und wieder der Universität Wien zur Verfügung stand.

\section{Standort gefunden, Übersiedlung aufgeschoben}

Bei der Vergabe der Stockwerke der Rotunde wurde die Eingangsebene 2 für die Fachbereichsbibliothek vorgesehen, wobei sowohl Kapazität als auch Barrierefreiheit für die Entscheidung maßgeblich waren. Im Zuge dessen wurde auch mit dem Institut ein Konsens getroffen, die Videothek zukünftig in der Bibliotheksebene anzusiedeln. Im Anschluss an eine sehr produktive, kurze Planungsphase im Frühjahr 2014 hätten eigentlich die Umbauarbeiten in der Bibliotheksebene beginnen sollen, stattdessen erfuhren sie einen Aufschub auf 
unbestimmte Zeit. Die Fachbereichsbibliothek musste daher vorerst an ihrem Standort in der Hofburg ausharren, während das Institut bereits im Herbst 2014 in die renovierten Räumlichkeiten in die Rotunde übersiedeln konnte. Eine zwei Jahre dauernde Wartezeit begann, in der die Bibliothek räumlich endgültig an ihre Kapazitätsgrenzen gelangte. Als im Herbst 2016 die Pläne für die Umbauarbeiten und die Übersiedlung endlich wieder aufgenommen werden konnten, war die Erleichterung dementsprechend groß.

Zur selben Zeit gelang es, getragen durch die Unterstützung der Direktorin der Universitätsbibliothek und der Dekanin der Philologisch-Kulturwissenschaftlichen Fakultät, nach ausgedehnten Verhandlungen mit dem Institut festzulegen, dass das Archiv der Theater-, Film- und Medienwissenschaft gemeinsam mit der Fachbereichsbibliothek in die Rotunde übersiedelt wird. Damit wurden den bereits seit Beginn der Pläne für eine Übersiedlung im Jahr 2013 geäußerten Wünschen entsprochen, Bibliothek und Archiv als Einheit zu sehen und auch zukünftig gemeinsam zu verorten.

\section{Same but different - Die neue Bibliothek}

Ende des Jahres 2016 begannen die umfangreichen Umbau- und Adaptierungsarbeiten. Gemäß den unveränderten Plänen aus dem Jahr 2014 wurden die baulichen Vorgaben der Fachbereichsbibliothek umgesetzt und zusätzlich um die notwendigen Angleichungen für das Archiv erweitert. So wurde der Eingangsbereich vergrößert, um ausreichend Platz für Informationsschalter und Benutzer_innen zu schaffen. Die vorhandenen Büroeinheiten auf der Nordostseite wurden geöffnet und zu einem großräumigen halbrunden Lesesaal verbunden. Ebenso bedurfte der Raum für das geplante Kompaktus-Regal für die Aufstellung der Bibliotheksbestände einer ausreichenden baulichen Anpassung. Bis Ende März 2017 waren die Bauarbeiten weitgehend abgeschlossen. Im April war es dann endlich soweit und die Übersiedlung der Fachbereichsbibliothek Theater-, Film- und Medienwissenschaft gemeinsam mit dem Archiv und der Videothek aus der Hofburg in die Rotunde konnte beginnen. Mit großem Engagement wurde das Projekt von allen Mitarbeiter_innen und Beteiligten unter exakter Einhaltung des Zeitplans erfolgreich durchgeführt. Bereits Anfang Mai öffnete die Fachbereichsbibliothek wieder ihre Türen für die allgemeine Benutzung.

Als Lernort stellt die Fachbereichsbibliothek einen wichtigen Faktor im Studienalltag dar. Bei der Planung des neuen Standorts stand daher die Berücksichtigung von unterschiedlichen räumlichen Lernzonen im Vordergrund. Ziel war es, ein Arbeitsumfeld zu schaffen, welches die individuellen Lernprozesse fördern und unterstützen soll. Dafür stehen neben dem Lesesaal auch die 
Bibliothekslounge und zwei Gruppenarbeitsräume zur Verfügung. Die stetig steigenden Benutzer_innenzahlen zeigen deutlich, dass dieses Mehrangebot an unterschiedlichen Arbeitssituationen von den Leser_innen sehr gut angenommen wird. Im Herbst 2018 konnten zudem die Öffnungszeiten der Fachbereichsbibliothek erweitert werden, mit positiven Auswirkungen für eine noch entspanntere Arbeitsatmosphäre in allen Bereichen. Die räumliche Nähe der Fachbereichsbibliothek zum Institut garantiert wieder optimale Bedingungen für die Unterstützung von Studierenden und Forscher_innen mit wissenschaftlichen Materialien und Medien. Mit der Aufstellung der Bibliotheksbestände im Freihandbereich sind zudem sämtliche Bücher und Zeitschriften für die Benutzer_innen zugänglich gemacht geworden.

\section{Bibliothek und Archiv}

Der Beschluss, die Fachbereichsbibliothek und das Archiv an den neuen Standort gemeinsam zu übersiedeln, ermöglichte es zum ersten Mal, alle vorhandenen Materialien des Archivs ordnungsgemäß zu verwahren und zumindest in Teilen zu ordnen. Durch diese Ordnung und grobe Klassifizierung konnten ausgewählte Teilbereiche gesichtet, bearbeitet und verzeichnet werden. Mit einem Ausstellungsprojekt "Völlig fraglich " ${ }^{1}$ wurde im November 2018 eine Brücke zu den ehemaligen Räumlichkeiten in der Wiener Hofburg geschlagen. Bereits vor zehn Jahren, 2008, wurde eine Ausstellung zur nationalsozialistischen Gründungsgeschichte des Instituts, der Bibliothek und des Archivs Theater-, Film- und Medienwissenschaft "Wissenschaft nach der Mode ${ }^{2}$ gestaltet, wobei damals die Instituts- und Bibliotheksräume in der Hofburg als Erinnerungsort fungierten. Nun wurde auch der neue Standort zur Projektionsfläche von Instituts- und Bibliotheksgeschichte.

Die Übersiedlung der Fachbereichsbibliothek, des Archivs und der Videothek für Theater-, Film- und Medienwissenschaft stellt somit ein gelungenes Beispiel für die hervorragende Kooperation unterschiedlicher Institutionen im universitären Umfeld dar. Durch die Zusammenlegung der Institutionen profitieren

1 Völlig fraglich. Vergessene Geschichte. Dauerausstellung im Institut und in der Fachbereichsbibliothek Theater-, Film- und Medienwissenschaft, UZA II, Rotunde. In: Website Institut für Theater, Film- und Medienwissenschaft, News, https://tfm.univie.ac.at/news/details/ news/voellig-fraglich-vergessene-geschichte-1/?tx_news_pi1\%5Bcontroller\%5D $=$ News \& tx _

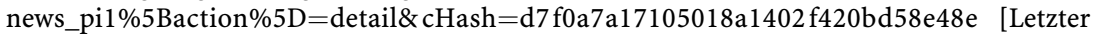
Zugriff: 25.06.2019].

2 Birgit Peter, Martina Payr (Hg.): „Wissenschaft nach der Mode«? Die Gründung des Zentralinstituts für Theaterwissenschaft an der Universität Wien 1943 [Ausstellung, 7. Mai bis 30. September 2008, Institut für Theater-, Film- und Medienwissenschaft, Hofburg, Batthyanystiege]. Wien u. a.: Lit 2008 (Universitätsgeschichte 3). 
nicht nur die Benutzer_innen von den Synergieeffekten in ihrem Studien- und Forschungsalltag, sondern auch die Fachbereichsbibliothek, das Archiv und die Videothek. Als weiterer Wissensraum wird der neue Standort die Universitätsbibliothek sicherlich auch in Zukunft bereichern. 


\section{El universo (que otros llaman la Biblioteca) ...' Die Fachbereichsbibliothek Theologie}

Zwar kann sie sich weder mit Borges' La biblioteca de Babel noch mit jenen Bibliotheken vergleichen, "zu denen ich Zugang hatte, da sie auch nachts geöffnet sind - nämlich die des Assurbanipal in Ninive, die des Polykrates auf Samos, die des Peisistratos in Athen, die von Alexandria (die schon im dritten Jahrhundert v. Chr. 400.000 Bände enthielt und dann im ersten, mit der des Serapeions, 700.000 Bände umfaßte), schließlich die Bibliothek von Pergamon und die des Augustus « ${ }^{2}$, weder ist sie »ein neugotisches Kloster « ${ }^{3}$ noch »ein Meisterwerk der modernen Architektur " ${ }^{4}$, in ästhetischer Hinsicht alles andere als das, vielmehr gerade das Gegenteil davon, und doch ist die Fachbereichsbibliothek Theologie »ein Modell des Universums ... ein dem Menschen gemäßes Universum ... auch mit der Möglichkeit, dass Studentenpärchen einen Nachmittag lang ... sitzen können, nicht um sich dort abzuknutschen, sondern um einen Teil ihres Flirts zwischen Büchern auszuleben, Büchern von wissenschaftlichem Interesse, die sie sich aus den Regalen holen und wieder zurückstellen. Mit einem Wort: eine lustvolle Bibliothek «. ${ }^{5}$

Der Urknall der Entstehung dieses menschengemäßen, modellhaften sowie lustvollen theologischen Universums fällt in die Direktionszeit von Maria Seissl und stellt eine markante Zäsur in der beinahe 200-jährigen Geschichte der evangelischen (gegründet 1821) und 635-jährigen Geschichte der katholischen (gegründet 1384) Bibliothek dar - am 21. Juni $2007^{6}$ erfolgte die feierliche Eröffnung der in die Schenkenstraße 8-10 übersiedelten und organisatorisch zur "Fachbereichsbibliothek Katholische und Evangelische Theologie» zusammengelegten ehemaligen Fakultätsbibliotheken für Evangelische bzw. Katholi-

\footnotetext{
1 Jorge Luis Borges: La biblioteca de Babel. In: Ders.: Ficciones, S. 38, https://www.ddooss.org/ libros/Jorge_Luis_Borges_ficciones.pdf [Letzter Zugriff: 03.01.2019].

2 Umberto Eco: Die Bibliothek. München, Wien: Hanser 1987, S. 11.

3 Ebd., S. 21.

4 Ebd.

5 Ebd., S. 38.

6 Der Betrieb wurde bereits mit Februar 2007 aufgenommen.
} 
sche Theologie. Die lange Bezeichnung sollte dabei den Wünschen nach konfessioneller Sichtbarkeit entgegenkommen. Überdies hatte man sich aufgrund der Größe des Bestandes und der Tatsache, dass zum Übersiedlungszeitpunkt etwa nur ein Drittel des Gesamtbestandes im Online-Katalog nachgewiesen war, dazu entschlossen, »die bisherigen Aufstellungssystematiken beizubehalten. Die Aufstellung des Bestandes nach einer evangelischen und einer katholischen Systematik signalisiert zudem den Respekt vor der jeweiligen dahinterstehenden kirchlichen Tradition und will ein ökumenisches Zeichen von Einheit in Vielfalt sein. Eine verhältnismäßig geringe Zahl an Dubletten bezeugt diese Vielfalt«. ${ }^{7}$

Allerdings stellten sich zehn Jahre später zwei Fragen erneut: (1) Da seit 2017 auch für das Institut für Islamisch-Theologische Studien die wissenschaftliche Literatur besorgt wird, wurde im selben Jahr die Bezeichnung zur Kurzform Fachbereichsbibliothek Theologie geändert. (2) Komplexer stellte sich die Problematik der systematischen Aufstellung dar: Durch den zunehmenden konfessionellen Pluralismus im christlichen Bereich (neben den der evangelischen und katholischen Kirche zugehörenden Studierenden zählen auch den orthodoxen sowie altorientalischen Kirchen angehörende Studierende zum Benutzerkreis; Einführung des Masterstudiums Religionspädagogik mit Schwerpunkt Orthodoxe Theologie an der Katholisch-Theologischen Fakultät) ließ sich der bilaterale ökumenische Aspekt nicht mehr realisieren. Dabei wäre auch grundsätzlich zu fragen, ob die Aufstellung der Bücher einer - wenngleich theologischen - Bibliothek überhaupt theologische Inhalte widerspiegeln soll oder nicht vielmehr sachlichen bibliothekarischen Prinzipien und Erfordernissen folgen sollte.

Das zweite akute Problem betraf den immer kostbarer werdenden Magazinsraum, der bei akzessorischer bzw. kursorischer Aufstellung besser ausgenutzt werden kann; zudem können die Bücher im Magazin nur an einer einzigen Stelle stehen, obwohl sie ihrem Inhalt nach oft an mehreren Systemstellen berücksichtigt werden müssten. ${ }^{8}$ Die zuletzt genannte Schwierigkeit lässt sich im Magazin nicht, im Katalog jedoch sehr leicht beheben, da im elektronischen Katalog die plurale sachliche bzw. klassifikatorische Erschließung der Literatur unbeschränkt möglich und an allen Systemstellen auffindbar ist. Des Weiteren lässt sich in einem solchen Katalog der aktuelle Bestand viel genauer abbilden als

7 Wolfgang Nikolaus Rappert (Text), Martin Hrabe (Fotos): Die neue Fachbereichsbibliothek Katholische und Evangelische Theologie der Universitätsbibliothek Wien. In: Mitteilungen der Vereinigung österreichischer Bibliothekarinnen \& Bibliothekare 60 (2007), H. 4, S. 56-58, hier S. 57.

8 Wieland Schmidt: Offene Buchbestände in Universalbibliotheken. In: Aktuelle Probleme der Bibliotheksverwaltung. Festgabe Hermann Fuchs zum siebzigsten Geburtstag am 13. März 1966 von Freunden und Schülern dargebracht. Hg. von Ewald Wagner. Wiesbaden: Harrassowitz 1966, S. 125-142. Insbesondere S. 127 f., S. 131 sowie S. 141. 
am Regal, da in ihm beispielsweise sowohl die elektronischen Medien als auch die entlehnten Werke verzeichnet sind (Maria Seissl pflegt mit Recht regelmäßig darauf hinzuweisen, dass gute und aktuelle Literatur nicht in der Bibliothek zu finden, sondern entlehnt sei). Aus diesen und anderen Gründen erfolgt $2019 \mathrm{mit}$ dem Übergang zur Aufstellung nach dem numerus currens die "Abkehr vom `Dogma der systematischen Aufstellung " " ${ }^{9}$ und hält somit wissensorganisatorisch die Moderne Einzug in die Fachbereichsbibliothek Theologie, da die virtuelle plurale systematische Zuordnung nicht vom physischen Objekt, sondern von der Vergabe sinnvoller Metadaten durch die Formal- und Sacherschließung abhängt. $^{10}$

In den Zeitraum von Maria Seissls Leitung fallen auch, abgesehen von kleineren, teilweise wertvollen Buchschenkungen, vor allem drei recht umfangreiche Schenkungen ganzer Bibliotheken:

- 2009 durch das Thomaskolleg der Erzdiözese Wien die ca. 10.000 Bände umfassende Frintaneumsbibliothek, deren Bestand 2012 nach Sprachen und Fachgebieten statistisch ausgewertet wurde ${ }^{11}$;

- 2012 der philosophische Nachlass des Systematikprofessors Falk Wagner, der 2019 bearbeitet wurde ${ }^{12}$;

- 2016 durch Sigrid Mühlberger die am 29. November 2018 feierlich eröffnete Privatbibliothek von Oberin Christine Gleixner (ca. 100 Laufmeter), deren ökumenische Abteilung 2018 aufgearbeitet wurde. ${ }^{13}$

Zum Bestand der Fachbereichsbibliothek Theologie gehört auch die europaweit einzigartige "Quellenbibliothek« des Instituts für Religionswissenschaft der Katholisch-Theologischen Fakultät, die Lukas Pokorny, Professor für Religionswissenschaft, seit 2017 aufbaut und in der originalsprachliche Primärschriften (sowie deren englische und deutsche Übersetzungen) einer Vielzahl neuer und alternativer religiöser Gruppen und Traditionen, die größtenteils

9 Thomas Hilberer: Numerus currens und iPod - die Organisation von Information mittels Metadaten und die Aufgabe der Bibliotheken im digitalen Zeitalter oder Die Kraft der digitalen Ordnung. In: Die Kraft der digitalen Unordnung. 32. Arbeits- und Fortbildungstagung der ASpB e.V. Sektion 5 im Deutschen Bibliotheksverband, 22. bis 25. September 2009 in der Universität Karlsruhe. Hg. von Jadwiga Warmbrunn und Jürgen Warmbrunn. Karlsruhe: KIT Scientific Publishing 2011, S. 103-111, hier S. 105.

10 Ebd., S. 104.

$11 \mathrm{https}: / /$ bibliothek.univie.ac.at/fb-theologie/frintaneumsbibliothek.html [Letzter Zugriff 11.09.2019].

$12 \mathrm{https} / / /$ bibliothek.univie.ac.at/fb-theologie/nachlassbibliothek_wagner.html [Letzter Zugriff 30.10.2019].

$13 \mathrm{https}$ ://bibliothek.univie.ac.at/fb-theologie/oberin-gleixner-bibliothek.html [Letzter $\mathrm{Zu}$ griff 11.09.2019]. 
auch Teil der religiösen Geschichte und Gegenwartskultur Österreichs sind, versammelt sind.

Maria Seissl ist es zu verdanken, dass die Fachbereichsbibliothek Theologie nicht nur keine "gute Bibliothek im Sinne einer schlechten Bibliothek ${ }^{14}$ ist und die neunzehn Kriterien dafür nicht erfüllt, sondern auch einen wesentlichen Aspekt von Ecos Utopie realisiert: »und es ist vor allem wichtig, daß die Leser direkten Zugang zu den Regalen haben ${ }^{15}{ }^{15}$

14 Eco 1987, S. 15.

15 Ebd., S. 38f. 


\section{Aus 5 mach 1 - bibliothekarisches »Hexen-Einmal-Eins «? Die neue Fachbereichsbibliothek Wirtschaftswissenschaften und Mathematik}

\section{Zusammenlegung}

Die Fachbereichsbibliothek Wirtschaftswissenschaften und Mathematik vereinigt die drei ehemaligen Standorte der damaligen Fachbereichsbibliothek Mathematik (Mathematik, Statistik, Mathematische Logik) sowie die zwei Standorte der einstigen Fachbereichsbibliothek Wirtschaftswissenschaften (Betriebswirtschaftslehre und Volkswirtschaftslehre) zu einem gemeinsamen Standort. Durch die Zusammenlegung zählt die »neue Fachbereichsbibliothek" mit insgesamt elf Mitarbeiter_innen (Teilzeit und Vollzeit, inklusive drei studentischen Mitarbeiter_innen) und einem Bestand von ca. 300.000 Medieneinheiten zu den größten Fachbereichsbibliotheken der Universität Wien. Zusätzlich beherbergt die Fachbereichsbibliothek auch ausgelagerte ZeitschriftenBestände der Österreichischen Zentralbibliothek für Physik, den KinderbuchBestand, Teile des Bühler-Nachlasses sowie den Nachlass Karl Kreil und die Historische Sammlung Mathematik.

Die Zusammenlegung der fünf Bibliotheken mit ihren unterschiedlichen »Aufbewahrungskulturen", Nutzer_innengruppen, Aufstellungssystematiken, etc., zu einer gemeinsamen modernen, nutzerfreundlichen Bibliothek am neuen Standort Oskar-Morgenstern-Platz war sicher eine der größten Herausforderungen in den letzten Jahren. Ziel war es, von außen als eine strukturierte Einheit - als eine Bibliothek - wahrgenommen zu werden. Gleichzeitig sollten gewisse Besonderheiten der fünf alten Standorte erhalten bleiben, ohne dass die neue Bibliothek dabei "zusammengewürfelt» oder »chaotisch« erscheint.

Der Umzug fand im Sommer 2013 statt. Die Vorbereitungsarbeiten, wie beispielsweise Dublettenkontrolle der Zeitschriften-Bestände, Ausscheiden von Medieneinheiten, Sichern der Bücher mit den neuen RFID-Strichcodes, begannen aber bereits Anfang 2012. 


\section{Bibliotheksräume}

Der Bibliothek wurden in einem neu adaptierten Gebäude Räumlichkeiten im Erdgeschoss, im ersten und im zweiten Untergeschoss zugewiesen. Im Erdgeschoss befinden sich die Büros der Mitarbeiter_innen, ein kleiner Lesesaal, eine Selbstverbuchungsanlage mit RFID-Technologie, Garderoben und der Informationsschalter.

Im ersten Untergeschoss befinden sich neben der Lehrbuchsammlung und der Mathematik-Schulbuch-Sammlung ein großer Lesesaal, eine halblaute Zone, drei Gruppenarbeitsräume (inklusive White-Boards) und ein eigener PC-Raum. Durch die Zusammenlegung wurden einerseits mehr Arbeitsplätze für die Benutzer_innen geschaffen - insgesamt gibt es in der neuen Bibliothek 215 Lernplätze -, und andererseits bietet die neue Bibliothek auch ganz unterschiedliche Möglichkeiten des Lernens: die beiden Lesesäle ermöglichen ruhiges, konzentriertes Lernen, die Gruppenarbeitsräume die gemeinsame Arbeit in Kleingruppen oder auch das Üben von Präsentationen, Referaten oder Vorträgen und die halblaute Zone, ausgestattet mit gemütlichen Polstermöbeln, ermöglicht einen Austausch und Diskussionen mit Studienkolleg_innen.

Obwohl ein Großteil der Arbeitsplätze im ersten Untergeschoss künstliche Beleuchtung benötigt, ist die Bibliothek mit ihren langen Öffnungszeiten (werktags bis $22 \mathrm{Uhr}$ und samstags bis $18 \mathrm{Uhr}$ ) für die Studierenden zu einem beliebten Lernort geworden.

\section{Literaturauswahl und Bestand}

Die Bibliothek hat den Anspruch, der wissenschaftlichen Forschung und Lehre gerecht zu werden. Die Fachbereiche, die bei verschiedenen Universitäts-Rankings oftmals vordere Plätze belegen, erfordern hinsichtlich der Literaturauswahl in elektronischer und gedruckter Form sorgfältige Betreuung. Da diese Bereiche stark forschungsorientiert und international ausgerichtet sind, ist es wichtig, sich auch auf fachlicher Ebene weiterzubilden und auf dem Laufenden zu bleiben, um neue Forschungsschwerpunkte im Bestand abbilden zu können. Die vielen Fernleihe-Anfragen aus dem In- und Ausland zeigen auch, dass die aktuellen Nachfragen aus dem Bereich der Wissenschaft gut abgedeckt werden. Für die Forschung ebenfalls von großer Bedeutung sind die Nachlässe des Fachbereiches Mathematik (z. B. Wiener Kreis) und der Nachlass Böhm-Bawerk aus dem Fachbereich Volkswirtschaftslehre.

Der gesamte Bestand der Fachbereiche Betriebswirtschaftslehre, Mathematik und Statistik ist bereits im Bibliothekssystem elektronisch erfasst. Seit 2011 werden die »Bücher vor 1945« aus dem Fachbereich Volkswirtschaftslehre und 
seit 2012 aus dem Fachbereich Mathematische Logik retrokatalogisiert. Bis voraussichtlich 2020 wird die Retrokatalogisierung abgeschlossen und alle Ressourcen somit elektronisch erfasst sein.

\section{Aufstellung}

Die Bestände - mit Ausnahme der Zeitschriften und der alten und wertvollen Bücher - sind zum überwiegenden Teil im zweiten Untergeschoss auf 10.000 Regal-Laufmetern untergebracht und für Benutzer_innen frei zugänglich. Sie sollten daher übersichtlich und geordnet präsentiert werden.

Die fünf zu übersiedelnden Bibliotheken hatten unterschiedliche Aufstellungssystematiken, teils thematisch fein gegliederte Haussystematiken (Betriebswirtschaftslehre und Volkswirtschaftslehre), teils Numerus-Currens-Aufstellungen (Mathematik, Mathematische Logik und Statistik). Die einzelnen Systeme beizubehalten und weiterzuführen erschien als zu unübersichtlich und nicht nutzerfreundlich, weshalb man sich für eine Vereinheitlichung nach einem gemeinsamen, neuen Numerus-Currens-System entschied.

Die Fachbereiche BWL, VWL, Statistik und Mathematische Logik sind bereits nach dem neuen gemeinsamen System aufgestellt und farblich unterschiedlich gekennzeichnet. Im Fachbereich Mathematik wurde auf Wunsch des Instituts für Mathematik vorläufig das alte System beibehalten. In den nächsten Jahren hat sich die Bibliothek zum Ziel gesetzt, die Bestände dieses Fachbereichs ebenfalls in das neue Numerus-Currens-System einzugliedern.

Ausgenommen von der farblichen Trennung der Fachbereiche ist die Lehrbuchsammlung. Die Fachbereichsbibliothek Wirtschaftswissenschaften und Mathematik ist eine der wenigen Standorte mit einer eigenen Lehrbuchsammlung vor Ort, deren Schwerpunkt Lehrbücher der BWL sind.

Die Reaktionen der Benutzer_innen auf die Numerus-Currens-Aufstellung sind sehr positiv. Aufgrund der farblichen Kennzeichnung und der einfachen Aufstellung ist eine relativ schnelle Orientierung im doch weitläufigen Magazin möglich. Da die Literatur formal und inhaltlich (teilweise sogar mit Notationen der Dewey Decimal Classification oder der Mathematics Subject Classification versehen) bestens erschlossen ist, bereitet auch die gezielte Suche zu einem bestimmten Thema in den Online-Systemen den meisten Benutzer_innen keine Schwierigkeiten. Eine thematische "Suche am Regal«, wie an den ehemaligen Standorten, wird in der neuen Bibliothek kaum mehr verlangt. 


\section{Team}

Wie die Bibliothek selbst, hat sich auch für das Team der Fachbereichsbibliothek vieles geändert. Die vorherigen Workflows wurden vereinheitlicht und den neuen Gegebenheiten angepasst. Alte Aufgabenbereiche fielen weg, viele neue kamen hinzu. Auch die Zusammensetzung des Teams hat sich in den letzten Jahren stark verändert. Viel internes Wissen über die Abläufe ist durch die Pensionierungen der beiden ehemaligen Leiter der früheren Fachbereichsbibliothek Mathematik Dominik Schwabl und der ehemaligen Fachbereichsbibliothek Wirtschaftswissenschaften Josef Friedl und den plötzlichen und viel zu frühen Tod von Brigitte Schiegl verloren gegangen.

Das Team ${ }^{1}$ hat in den letzten Jahren stets Neues dazugelernt bzw. dazulernen müssen, sich fachlich verbessert und »zsammgrauft«. Jedenfalls ist es für sämtliche Herausforderungen, die in den nächsten Jahren auf die Bibliothek zukommen werden, bestens gerüstet. Die Jubilarin dieses Bandes, Maria Seissl, hat es als Leiterin der Dienstleistungseinrichtung Bibliotheks- und Archivwesen geschafft, dass sich trotz der Besonderheiten und Unterschiede der vielen Abteilungen und Fachbereichsbibliotheken alle Mitarbeiter_innen als »die UB« fühlen und hier im Kleinen bemühen auch wir uns täglich, »aus fünf eins zu machen $«$.

1 Derzeit gehören dem Kernteam folgende Personen an: Andrea Bakosch, Margit Cerny, Julio Estevez, Gerald Ferenz, Gabriela Freisehner, Günther Müller, Andrea Neidhart, Martina Svetlik und Christine Scheich. 


\section{Die »Videosammlung Stadtkino « als Teil des österreichischen televisuellen Gedächtnisses an der Fachbereichsbibliothek Zeitgeschichte}

Gegenstand der Zeitgeschichte ist die soeben vergangene Geschichte. Die "Epoche der Mitlebenden und ihre wissenschaftliche Bedeutung ${ }^{1}$ ist dabei je nach Ausrichtung der Forschungsinstitution statisch und/oder dynamisch zu verstehen und durch die Herausbildung von neuartigem Quellenmaterial (Fotografie, Hörfunk, Television, Internet, Soziale Medien, ...) sowie einhergehend mit neuen Methoden, wie etwa Oral History, gekennzeichnet. Audiovisuelle Medien sind als filmische und televisuelle Repräsentationen der Zeit- und Kulturgeschichte ein wesentliches Material der zeithistorischen Forschung. Daher war und ist es auch immer ein Anliegen der Zeitgeschichte, diese Medienformen und deren Inhalte zu sammeln und zu archivieren.

Gleichzeitig stellt das Sammeln, Bewahren, Erschließen und Bereitstellen bzw. Herausgeben - sei es in analoger oder digitaler Form - einen für die Gesellschaft und die Wissenschaft relevanten Auftrag dar, der neben Archiven und Museen auch von wissenschaftlichen Bibliotheken zu erfüllen ist. Diese erweiterte Perspektive des bibliothekarischen Handelns soll, trotz des primären Fokus einer wissenschaftlichen Bibliothek auf die Informationsversorgung der Wissenschaft in Forschung, Lehre und Studium, anhand der Videosammlung des Wiener Stadtkinos gezeigt werden.

\section{Zeitgeschichte und Medienarchive}

Einhergehend mit der Herausbildung von institutioneller zeitgeschichtlicher Forschung in den 1960er Jahren in Österreich wurde begonnen, sich zeitgeschichtlich und bibliothekarisch mit dem Thema AV-Medien zu beschäftigen,

1 Hans Rothfels: Zeitgeschichte als Aufgabe. In: Vierteljahreshefte für Zeitgeschichte 1 (1953), H. 1, S. 1-8, hier S. 4. 
wobei auch die fachliche Betreuung und Beratung von Projekten im Bereich Funk, Film und Fernsehen zu den Aufgaben zählte und zählt. ${ }^{2}$

In Folge entstanden dabei Kooperationen des Instituts für Zeitgeschichte der Universität Wien mit wesentlichen heutigen Bestandsträgern des österreichischen audiovisuellen Gedächtnisses, wie etwa dem Archiv des Österreichischen Rundfunks (ORF), dem Bildarchiv der Österreichischen Nationalbibliothek und der Österreichischen Mediathek im Technischen Museum Wien. Mitunter wurden auch große und bedeutende eigene Sammlungen angelegt, wie etwa das Bildarchiv der Österreichischen Gesellschaft für Zeitgeschichte, das auch aus konservatorischen Gründen 2009 an die Österreichische Nationalbibliothek übergeben wurde.

Seit 2011 sorgt eine Kooperation mit dem ORF für nationale und internationale Aufmerksamkeit. Dem Institut für Zeitgeschichte gelang es, über eine Recherche-Station an der Fachbereichsbibliothek Zeitgeschichte erstmals eine externe Zugangsmöglichkeit zu dem seit 1955 gesendeten und digital vorhandenen Material des ORF-Archivs für die Studierenden und Wissenschafter_innen der Universität Wien anzubieten und über einen Kooperationsvertrag auch Digitalisierungen für wissenschaftliche Projekte anstoßen zu können. ${ }^{3}$

\section{Übergabe der Videothek des Stadtkinos}

Dabei verfügt die Fachbereichsbibliothek Zeitgeschichte selbst über eine durchaus respektable AV-Sammlung, deren Inhalte (noch) nicht durchgängig durch Streamingdienste, Video-Plattformen etc. abgedeckt werden können. Als Ergänzung dieses AV-Schwerpunktes wurde im Herbst 2013 die Videosammlung des Stadtkinos Wien im Zuge dessen Übersiedlung vom Schwarzenbergplatz ins Künstlerhaus am Karlsplatz übernommen. Das 1916 eröffnete Schwarzenbergkino (später Kammerlichtspiele) - eines der ältesten Programmkinos Wiens ${ }^{4}$ -

21961 wurde von der Österreichischen Gesellschaft für Zeitgeschichte (ÖGZ) zunächst das Österreichische Institut für Zeitgeschichte begründet, das 1966 schließlich als Institut für Zeitgeschichte der Universität Wien eingerichtet wurde. Die Bestände beider Institutionen bilden den Grundstock der Ende 1982 als Teilbibliothek der Universitätsbibliothek Wien reorganisierten Fachbibliothek für Zeitgeschichte, die seit 2006 als Fachbereichsbibliothek Zeitgeschichte (FbZ) firmiert. Vgl. Markus Stumpf: Die Fachbereichsbibliothek Zeitgeschichte der Universität Wien im Spiegel ihrer Bestands- und Benutzungsgeschichte. In: Bertrand Perz, Ina Markova (Hg.): 50 Jahre Institut für Zeitgeschichte der Universität Wien 1966-2016. Wien: New Academic Press 2017, S. 168-189.

3 Vgl. https://bibliothek.univie.ac.at/fb-zeitgeschichte/orf-archiv.html [Letzter Zugriff: 22.08. 2019].

$4 \mathrm{Zu}$ den Anfängen des Wiener Kinos vgl. u. a. Werner Michael Schwarz: Kino und Kinos in Wien. Eine Entwicklungsgeschichte bis 1934. Wien: Turia \& Kant 1992. 
wurde 1981 als kommunales Kino mit eigenem Filmverleih unter dem Namen Stadtkino neu gegründet. Als Eröffnungsfilm wurde Zechmeister (1981) von Angela Summereder, ein poetisch-experimenteller Versuch, einen ungeklärten Gattenmord aus den Jahren nach dem Zweiten Weltkrieg zu rekonstruieren, gezeigt. ${ }^{5}$ Bereits nach einem Jahr wurde begonnen, einen eigenen Filmverleih sowie ein Archiv an Dokumentationen und Diskussionssendungen im Rahmen einer Videothek aufzubauen. ${ }^{6}$ Dazu waren Bestände vom Z-Club - die ehemalige Zentralsparkasse der Gemeinde Wien bemühte sich um ein modernes Image und hatte mit dem Z-Club 1975 der alternativen Szene Wiens eine Heimstätte geboten $^{7}$ - übernommen worden und so verfügte die Videothek bereits 1989 über etwa 1.000 Kassetten mit ORF-Produktionen, wie Club 2, Dokumentarfilmen, Porträts und historischen Sendungen. ${ }^{8}$ Diese Sammlung wuchs später auf etwa 2.800 Videokassetten an. Darin wurden von 1978 bis 2001 mehr als 7.000 Stunden an Sendungsmitschnitten aus dem ORF und anderer Fernsehsender aufgezeichnet und archiviert. Zusätzlich wurden Ausschnitte aus Programmzeitschriften, aber auch Rezensionen und andere Texte über die Sendungen, in Ordnern gesammelt. Insgesamt war der Platzbedarf der Videothek mit etwa 77 Laufmetern durchaus beachtlich.

Claus Philip, der damals designierte neue Leiter des Stadtkinos, wandte sich 2008 an Albert Müller (1959-2019), den Archivbeauftragten des Instituts für Zeitgeschichte, bezüglich einer Übernahme der Videosammlung des Stadtkinos. Diese wurde bei einer Besprechung mit Franz Schwartz, dem langjährigen Leiter des Stadtkinos, konkretisiert und im April 2009 auch die Fachbereichsbibliothek Zeitgeschichte involviert. Erst im November 2013, anlässlich der tatsächlichen Übersiedlung des Stadtkinos ins Künstlerhaus, wurde, nach Besichtigung und Zustimmung der Leitung der Universitätsbibliothek Wien, die Videosammlung samt Geräten und Indexmappen vom Schwarzenbergplatz an die Fachbereichsbibliothek Zeitgeschichte verbracht.

Die folgenden Sichtungs- und Sortierungsarbeiten sowie die Erstellung eines vorläufigen Index lieferten zwei Erkenntnisse: Erstens, dass die zur Einrichtung einer Sichtungsstation übernommenen Geräte des Videoformats Betamax nicht mehr fehlerfrei funktionierten. Eine Reparatur wäre zu teuer gekommen und die

5 Werner Fritz: 90 Jahre Kino und Film in Wien (1896-1986). In: 90 Jahre Kino und Film in Wien. 1896-1986. Vergangenheit und Zukunft. Hg. vom Verband der Wiener Lichtspieltheater und Audiovisionsveranstalter. Wien: Jugend \& Volk 1986, S. 31-46, hier S. 38.

6 Juliane Batthyány: Kinos in Wien. Vom Alltag und Überleben der kleinen Filmtheater. Erfurt: Sutton 2010, S. 61-70.

7 Vgl. https://www.geschichtewiki.wien.gv.at/Zentralsparkasse_der_Gemeinde_Wien [Letzter Zugriff: 06.06.2019].

8 Robert Holzbauer, Gerhard Jagschitz, Peter Malina: Handbuch audiovisueller Medien in Österreich. Wien: Arbeitsgemeinschaft Audiovisueller Archive Österreichs 1989, S. 25. 
am Markt verfügbaren funktionierenden Alt-Geräte wären nur zu unerschwinglichen Preisen zu erwerben gewesen. In Anbetracht der Tatsache, dass auch mit dem einmaligen Erwerb/Reparatur keine Nachhaltigkeit erzeugt worden wäre, fiel die strategische Grundsatzentscheidung, die notwendige Infrastruktur für dieses Format nicht zur Verfügung zu stellen. Dafür konnten die Betamax-Geräte an die Österreichische Mediathek des Technischen Museum Wien im November 2014 als Ersatzteile abgegeben werden. Zweitens, dass der Fachbereichsbibliothek Zeitgeschichte ein kulturhistorisch wertvoller televisueller Schatz übergeben worden war.

\section{Hebung eines televisuellen Schatzes}

Denn auf Basis des vorläufigen Index ${ }^{9}$ stellte sich rasch heraus, dass eine erstaunliche Anzahl von Mitschnitten von ORF-Sendungen aus den 1970/80er Jahren im ORF-Archiv selbst nicht vorhanden war. Einige Sendungen waren schlicht und einfach nicht aufgezeichnet oder - aus heute nicht mehr nachvollziehbaren Gründen - gelöscht worden.

Daher kam es bald zu ersten Gesprächen mit dem ORF und im Sommer 2015 wurde festgestellt, dass alleine bei den vorhandenen Aufzeichnungen im Format Betamax knapp 400 Sendungen, davon über 222 Zeit im Bild-Mitschnitte, mit insgesamt etwa 600 Aufnahmestunden des televisuellen kulturellen Erbes Österreichs durch die Videosammlung gerettet worden waren. Der ORF sorgte schließlich für deren Digitalisierung und in weiterer Folge für die Zugänglichkeit der Inhalte über den an der Fachbereichsbibliothek Zeitgeschichte eingerichteten externen Zugang zum ORF-Archiv.

Dabei stellte sich die Qualität der Aufnahmen in der Regel als akzeptabel heraus. "Als historische Dokumente mit all den technik- und zeitbedingten Fehlern kann man das Meiste noch immer im Fernsehen verwenden «, so Kurt Schmutzer vom ORF-Archiv. Was die Mitschnitte interessant und wertvoll macht, ist, dass es von den Moderationen der Zeit im Bild, der Werbung und der Programmansage (Ansager_innen) und Promotion kaum Aufzeichnungen gibt. Archiviert wurden über lange Zeit fast ausschließlich Sendungen und Beiträge, nicht aber das "Drumherum». D.h. im Archiv lassen sich von diesen Programmelementen bis hin zum Screendesign wenige Belege finden. Und die Mitschnitte des Stadtkinos ergänzen dies zumindest für einige Jahre sehr gut. Mit der Digitalisierung der gesamten Bänder wurden auch einige Dubletten von

9 Zum überarbeiteten Verzeichnis siehe Günter Bräuhofer, Markus Stumpf (Hg.): Findbuch der Videosammlung Stadtkino an der Fachbereichsbibliothek Zeitgeschichte der Universität Wien. Stand: 27. August 2019. DOI: https://doi.org/10.25365/phaidra.109. 
bereits im Archiv vorhandenen Sendungen produziert, "aber das ist die Sache schon wert. Besser manches doppelt als einiges gar nicht ... «, so Schmutzer. ${ }^{10}$

Im Dezember 2018 konnte die Digitalisierung abgeschlossen werden, sodass im ORF-Archivsystem »mARCO« diese Sammlung nun als »FBZG Stadtkino [Videonummer] « indiziert und auffindbar ist. Erfasst sind diese derzeit nur mit dem ersten Sendungstitel und dessen Sendedaten, sowie die Stadtkino-Nummer im Titel. ${ }^{11}$ Eine genauere Erfassung und Segmentierung der Files wird noch einige Zeit dauern. Die Fachbereichsbibliothek Zeitgeschichte ist damit nun auch im Gedächtnis des ORF eingeschrieben. Und sie erhält die vom ORF angefertigten Digitalisate, um die »Videosammlung Stadtkino" möglichst vollständig verfügbar zu haben. In einem Aussonderungskonzept wird sich die Fachbereichsbibliothek Zeitgeschichte nun mit der Frage, was von dieser Sammlung erhaltenswert ist, beschäftigen müssen. Dabei wird sich wohl auch noch der eine oder andere bislang nicht im ORF-Archiv vorhandene Mitschnitt finden lassen.

\section{Resümee}

Das Nebenprodukt »Dokumentation« des Stadtkinos zeigt sich heute als überraschender televisueller und kulturhistorisch relevanter Schatz, der von der Fachbereichsbibliothek Zeitgeschichte in Kooperation mit dem ORF-Archiv gehoben werden konnte. Die erweiterte Perspektive des bibliothekarischen Verständnisses und Handelns hinsichtlich eines für die Gesellschaft und für die Wissenschaft relevanten Kulturauftrags war dafür mitverantwortlich. Waren bereits die Akteur_innen des Stadtkinos selbst mit ihrer "Dokumentation" gegen eine erstaunliche und manchmal erschreckende Geschichtslosigkeit angetreten, so sieht sich heute auch das ORF-Archiv als Mittel zur »Vorbeugung gegen Gedächtnisverlust « ${ }^{12}$.

10 E-Mail Kurt Schmutzer an Markus Stumpf, 18.03.2019.

11 Eine Abfrage am 08.03.2019 ergab 224 Treffer.

12 Herbert Hayduck: Das Archiv. Ihre Vorbeugung gegen Gedächtnisverlust. In: Public Value Report 2017/2018. Der Unterschied. 55 Stimmen aus dem ORF zu öffentlich-rechtlicher Medienqualität. Wien: ORF 2018, S. 106-109. 
Open-Access-Publikation im Sinne der CC-Lizenz BY-NC-ND 4.0

(c) 2019, Vandenhoeck \& Ruprecht GmbH \& Co. KG, Göttingen ISBN Print: 9783847110989 - ISBN E-Lib: 9783737010986 


\section{Forschungsunterstützende Services und Projekte}


Open-Access-Publikation im Sinne der CC-Lizenz BY-NC-ND 4.0

(c) 2019, Vandenhoeck \& Ruprecht GmbH \& Co. KG, Göttingen ISBN Print: 9783847110989 - ISBN E-Lib: 9783737010986 


\section{Der Film in der Welt des Buches. Die AG AV-Medien im Unterricht an der Universitätsbibliothek Wien}

Die Arbeitsgruppe für audiovisuelle Medien im Unterricht, kurz AG AV-Medien, wurde 2010 im Auftrag des Medienservice des Bundesministeriums für Bildung, Wissenschaft und Forschung (BMBWF) ins Leben gerufen und ist damit ein für eine Bibliothek eher ungewöhnliches Projekt.

Das Medienservice ist eine Einrichtung der Republik Österreich, die schon seit den 1920er Jahren Schulen mit Filmen, audiovisuellen Medien und Medienbegleitmaterialien versorgt. Seit 2003 tut es dies nicht nur über den Verkauf von physischen Medien (z.B. DVDs), sondern auch mittels der Online-Streamingplattform Bildungsmedien.TV, die von der AG AV-Medien redaktionell betreut wird. Lehrende an österreichischen Schulen und an nicht kommerziellen Erwachsenenbildungseinrichtungen, sowie alle Mitarbeiter_innen und Student_innen an Universitäten und Pädagogischen Hochschulen können das Angebot für den Unterrichtsgebrauch kostenlos nutzen.

Vor 2010 war die Institution des BMBWF-Medienservice noch nicht entsprechend in der Lehrer_innenaus- und -fortbildung verankert. Mit der Integration des Mediums Film in die Welt des Bibliothek- und Archivwesens gelang nun gleichzeitig die Zusammenführung des schulischen und universitären Bereichs. Zwei traditionell getrennte Bereiche, die nicht immer leicht zueinander finden, wurden damit vereint.

Die Universität Wien war zwar schon immer die größte Einrichtung zur Lehrer_innenausbildung in Österreich, doch existierte dieser Bildungsbereich aufgeteilt in Universitäten und Pädagogische Hochschulen lange Zeit in zwei getrennten Welten. Durch die Kooperation der Pädagogischen Hochschulen mit den Universitäten soll diese Trennung überwunden werden. In diesem Sinn leistet auch die Verankerung der AG AV-Medien und der Streamingplattform Bildungsmedien.TV an der Universitätsbibliothek Wien einen wichtigen Beitrag.

Dieser ist umso wichtiger, da in einer vom Internet und Smartphone beherrschten Welt der Film als Bildungsmedium zunehmend an Bedeutung gewinnt. Gestreamte Filme aus dem Internet beherrschen den Alltag der Kinder und Jugendlichen und auch deren Lernstrategien. Denn nicht mehr nur text- 
basierte Internetseiten wie Wikipedia sind die »Lehrmeister « von heute, sondern immer häufiger übernimmt YouTube diese Aufgabe. YouTube als eine eher chaotische Ansammlung von über einer Milliarde Filmen wird dabei oft ohne didaktische Einbettung genutzt.

Eine Aufgabe der AG AV-Medien im Unterricht ist es, den Lehrenden eine Hilfestellung bei der Bewältigung dieser ungeordneten Flut an Information zu geben. Zudem stellt die Online-Streamingplattform Bildungsmedien.TV heute das wichtigste Instrument zur Verbreitung von AV-Unterrichtsmedien dar. Derzeit sind über 2.000 nach didaktischen Kriterien ausgewählte und übersichtlich sortierte AV-Medien (didaktische DVDs, Unterrichtsfilme, Dokumentationen, Audios, Bildserien, interaktive Lerntools u.v.m.) per Live-Streaming verfügbar. Alle Medien wurden von Lehrer_innen aus der Schulpraxis begutachtet bzw. in ihrer Entstehung begleitet. Die meisten Filme sind mit umfangreichem Begleitmaterial versehen, welches von Lehrer_innen erstelltes Arbeitsmaterial, Hintergrundinformationen, Linktipps und Stundenbilder enthält.

Die AG AV-Medien fungiert zudem als Beratungseinrichtung und kommunikative Schnittstelle zwischen dem BMBWF-Medienservice, Filmproduzent_innen, TV-Sendern, Lehrenden an Österreichs Schulen, Erwachsenenbildungseinrichtungen und in der Lehrer_innenausbildung. Sie verbindet somit Medienproduzent_innen und -konsument_innen und dient als Ansprechpartnerin für Lehrende unterschiedlicher Institutionen, sowie für Studierende von Lehramtsfächern zum Thema Film und Bildungsmedien.TV.

Die AG AV-Medien begleitet die Abwicklung von Unterrichtsfilmproduktionen für das BMBWF-Medienservice redaktionell von der Ideenfindung bis zur Bereitstellung des fertigen Unterrichtsmediums durch das BMBWF-Medienservice. Die Arbeitsgruppe arbeitet dabei eng mit Lehrerinnen und Lehrern, die als Gutachter_innen und Expert_innen für AG AV-Medien tätig sind, sowie dem BMBWF-Medienservice zusammen. Alle von Schulfilmfirmen angekauften und mit dem ORF gemeinsam produzierten Filme werden über Bildungsmedien.TV verfügbar gemacht.

Für diesen vielschichtigen Tätigkeitsbereich der AG AV-Medien als Schnittstelle zwischen Schule und Lehrer_innenausbildung, Ministerium und Filmfirmen, visueller Wissensvermittlung und didaktischen Aufbereitung, ist die Universitätsbibliothek Wien ein idealer Ort: Sie ist unter anderem eine reiche Quelle für die so häufig benötigten wissenschaftlichen und literarischen Texte. Umso mehr, da man auf die stets vorhandene sachkundige und freundliche Unterstützung durch die Mitarbeiter_innen der Universitätsbibliothek Wien vertrauen kann, welche die Arbeit in diesem komplexen Aufgabenbereich wesentlich erleichtert. 


\author{
Juan Gorraiz / Christian Gumpenberger / Ursula Ulrych / \\ Martin Wieland
}

\title{
Mehr als nur Erbsenzähler und Kaffeesudleser. Die Abteilung Bibliometrie und Publikationsstrategien im Zeitalter von »publish or perish « und Open Science
}

\section{Von einer Graswurzelbewegung zur eigenen Abteilung}

Bibliothekar_innen gelten allgemein ja gerne als langweilig, traditionell bis antiquiert. Es gibt allerdings kaum ein Berufsbild, das in den letzten Jahrzehnten so vielen dramatischen Veränderungen unterworfen war wie das des Bibliothekars/ der Bibliothekarin. Im Zeitalter der elektronischen Informationsgesellschaft gepaart mit einem zunehmend wettbewerbsorientierten wissenschaftlichen Umfeld müssen wissenschaftliche Bibliotheken blitzschnell auf internationale Trends und Herausforderungen reagieren und ihr Anforderungsprofil entsprechend flexibel anpassen. Bibliometrie und Szientometrie sind daher ideale Betätigungsfelder für moderne wissenschaftliche Bibliothekar_innen als elementarer Baustein einer modernen Forschungsunterstützung.

Die allerersten Analysen bibliometrischer Natur wurden sogar von Bibliothekaren ${ }^{1}$ durchgeführt und dienten primär einem optimierten Bestandsmanagement. Als neue Disziplin der Bibliotheks- und Informationswissenschaften entwickelte sich die Bibliometrie allerdings sukzessive zu einem Instrument für die Messung und Überwachung des wissenschaftlichen Outputs. Juan Gorraiz hat dies schon früh erkannt und bereits in den 1990er Jahren an der Universität Wien Pionierarbeit in Form erster bibliometrischer Analysen geleistet. Auf seine Initiative wurde 2007 die interinstitutionelle "Arbeitsgruppe Szientometrie« an der Universität Wien gegründet. Die bibliometrischen Aktivitäten wurden seitens der Direktion von Anfang an gefördert, aktiv unterstützt und 2009 schließlich als eigene Organisationseinheit innerhalb der Universitätsbibliothek Wien verankert. Die initial als "Team Bibliometrie» bezeichnete "one-and-ahalf-man show" hat sich über die Jahre hin zur "Abteilung Bibliometrie und Publikationsstrategien " entwickelt und ist sowohl aufgabenmäßig als auch personell sukzessive gewachsen. Dieser Erfolg wäre ohne die Offenheit der Bi-

1 P.L.K. Gross, E.M. Gross: College libraries and chemical education. In: Science 66 (1927), Nr. 1713, S. 385-389. 
bliotheksleitung gegenüber neuen Anforderungen an moderne Bibliotheken sowie dem dazu notwendigen Vertrauensvorschuss nicht möglich gewesen.

\section{»Service is our success«}

Die Abteilung Bibliometrie und Publikationsstrategien versteht sich an der Universität Wien primär als Serviceprovider und operiert hier als klassischer "Doppelagent«: Auf der einen Seite steht sie allen interessierten Forschenden bei allen bibliometrisch und publikationsstrategisch relevanten Fragen zur Verfügung, auf der anderen Seite kooperiert sie eng mit der Qualitätssicherung bei Individual- und Fakultätsevaluierungen sowie mit Berufungskommissionen bei Berufungsverfahren und liefert Expertise in Form von bibliometrischen Analysen. Die Nachfrage ist in den letzten Jahren eklatant gestiegen und weiter im Wachsen begriffen.

Der Abteilung ist es allerdings wichtig, die Bedeutung der Bibliometrie nicht nur auf den evaluativen Aspekt zu reduzieren, sondern den publikationsstrategischen Wert für die Wissenschafter_innen hervorzuheben. In Einzelberatungen, Seminaren und Workshops an Fakultäten, Lehrveranstaltungen für Doktorand_innen sowie in der Ausbildung des bibliothekarischen Nachwuchses wird regelmäßig auf die Wichtigkeit und praktische Anwendbarkeit von bibliometrischen Aspekten im wissenschaftlichen Alltag hingewiesen. Die Schlagkraft der Abteilung erhöht sich natürlich in einer engen Verschränkung mit den anderen forschungsunterstützenden Services der Universitätsbibliothek Wien, vor allem mit dem Team u:cris, dem E-Resource Management und dem Open Access Office.

\section{Nachfrage jenseits der eigenen universitären Grenzen}

Die Abteilung Bibliometrie und Publikationsstrategien ist mittlerweile weit über die Grenzen der Universität Wien hinaus bekannt und für ihr Knowhow geschätzt, weshalb dieses externen Interessent_innen auch gerne kostenpflichtig angeboten wird. Innerhalb der letzten Jahre hat die Abteilung bibliometrische Analysen, Expertisen, Konzepte und Beratungen für den FWF, den WWTF, die Ludwig Boltzmann Gesellschaft, die Max-Planck-Gesellschaft, die Universität Göttingen, die ETH Zürich und die Humboldt-Stiftung durchgeführt. Auf diese Art und Weise gehört die Bibliometrie zu den Organisationseinheiten der Bibliothek, die auch aktiv Drittmittel einwerben können. 


\section{Licht ins bibliometrische Dunkel}

Als Reaktion auf das Fehlen einer einschlägigen bibliometrischen Ausbildung (vor allem in Mitteleuropa) und auf die steigende Nachfrage (vor allem vom Forschungsmanagement) haben sich 2010 die Universität Wien, die HumboldtUniversität zu Berlin (Deutschland), das damalige Institut für Forschungsinformation und Qualitätssicherung - iFQ - (Deutschland) und die Katholieke Universiteit Leuven (Belgien) zusammengeschlossen, um kooperativ die »European Summer School for Scientometrics (esss) « ins Leben zu rufen. Das iFQ wurde mittlerweile in das DZHW (Deutsches Zentrum für Hochschul- und Wissenschaftsforschung) integriert, die Humboldt-Universität hat die Kooperation verlassen, dafür ist 2017 die Unversidad de Granada als neues Kooperationsmitglied dazugekommen. Die Geschäftsstelle der esss ist seit Beginn fix an der Bibliometrie in Wien verankert und hat es 2018 schließlich geschafft, die esss als »univie:summer school« akkreditieren zu lassen und erstmals ECTS-Punkte für die Teilnehmer_innen zu vergeben. Mittlerweile hat die esss bereits zum zehnten Mal (davon drei Mal in Wien) mit großem Erfolg und einer internationalen Beteiligung stattgefunden, die weit über die europäischen Grenzen hinausgeht.

\section{Internationale(r) Sichtbarkeit und Impact}

Die Abteilung Bibliometrie und Publikationsstrategien sieht sich nicht nur als reiner Provider von Service oder Lehrkompetenz, sondern auch als aktiv Teilnehmende am internationalen wissenschaftlichen Diskurs. Fachlich einschlägige Konferenzen wie die ISSI (International Society for Scientometrics and Informetrics) Conference und die STI (International S\&T Indicators) Conference sowie die QQML (Qualitative and Quantitative Methods in Libraries International) Conference sind regelmäßige Foren für die Vorstellung eigener Projekt- und Forschungsergebnisse. Eine Vielzahl an Publikationen in renommierten Journals wie Scientometrics, Journal of Informetrics, Journal of the American Society for Information Science and Technology, Research Evaluation, Frontiers in Research Metrics and Analytics, etc. dokumentieren eindrücklich, dass wissenschaftliche Bibliothekar_innen auch aktive Informationswissenschafter_innen sein können und sollen.

2 http://www.scientometrics-school.eu/ [Letzter Zugriff: 11.09.2019]. 


\section{Bibliometrie - quo vadis}

Im Zeitalter von Open Science ist es wichtig, dass die Bibliometrie auch am Puls der Zeit bleibt. Im Rahmen des HRSM-Projektes e-Infrastructures Austria hat die Abteilung Bibliometrie und Publikationsstrategien auch das Teilprojekt »Planung und Durchführung einer österreichweiten Umfrage zu Forschungsdaten« geleitet und den international beachteten Bericht »Forschende und ihre Daten « publiziert. 2018 ist es der Bibliometrie auch in Kooperation mit dem Team u:cris endlich gelungen, die Universitätsleitung von der Wichtigkeit eines "unique person identifier« zu überzeugen und nach langer Vorarbeit ORCID (Open Researcher and Contributor ID) in $\mathrm{u}$ :cris $\mathrm{zu}$ implementieren. Die Bibliometrie der Universität Wien hat ab 2019 gemeinsam mit der TU Wien den Lead für ein ORCID-AT Konsortium übernommen.

Auch neue Indikatoren jenseits des traditionellen Zitationsuniversums sind international auf dem Vormarsch. Die Bibliometrie verfolgt hier auch an der Universität Wien weiterhin ihr Interesse an der Exploration und Weiterentwicklung von alternativen Metriken (Altmetrics) und speziell an der Herausforderung quantitativer Methoden im Bereich der Geistes-, Kultur- und Sozialwissenschaften, welchen seit den letzten drei Jahren in mehreren Projekten (Sichtbarkeitssteigerung in den Geistes-, Kultur- und Sozialwissenschaften Teilprojekte 1-3) begegnet wird. 


\section{Der erfolgreiche Aufbau einer nationalen Dateninfrastruktur. AUSSDA - The Austrian Social Science Data Archive}

AUSSDA - The Austrian Social Science Data Archive befindet sich »im Aufbruch « seit 2016, jenem Jahr, in dem AUSSDA gegründet und als eine Core Facility an der Dienstleistungseinrichtung Bibliotheks- und Archivwesen der Universität Wien verankert wurde. Es war gleichzeitig der Start der dreijährigen Projektfinanzierung durch das Bundesministerium für Bildung, Wissenschaft und Forschung (BMBWF) und der Beginn einer spannenden Reise. Um sich als eine zentrale Forschungsinfrastruktur für sozialwissenschaftliche Daten in Österreich zu etablieren, wurde AUSSDA als Konsortium an den Universitäten Wien, Graz und Linz verankert. Auf internationaler Ebene besteht der Auftrag darin, Österreich im europäischen Verbund der sozialwissenschaftlichen Datenarchive (CESSDA ERIC) zu repräsentieren. Im Mission Statement werden die verschiedenen Aspekte der täglichen Arbeit verdeutlicht: »Wir machen sozialwissenschaftliche Daten zugänglich und nachnutzbar, für Wissenschaft und Gesellschaft."

Unter "zugänglich und nutzbar machen« fallen dabei so verschiedene Aufgaben wie Forschungsdaten akquirieren, Lizenzvereinbarungen treffen, Daten für die Nutzung durch Dritte bereitstellen und, soweit dies vereinbart und möglich ist, auch deren Aufbereitung. Weitere wichtige Arbeitsbereiche befassen sich unter anderem mit den rechtlichen Grundlagen der Archivierung und Bereitstellung von Daten, vor allem zum Thema Datenschutz, der langfristigen Archivierung von Daten, der Kommunikation und dem Marketing, der Aufrechterhaltung und Weiterentwicklung der technischen Infrastruktur sowie der Beratung und Schulungen zu Datenmanagementplänen. Bei der Zielgruppe gibt es die gesonderte Nennung Wissenschafter_innen, da diese die meisten Nutzer_innen von AUSSDA stellen - sowohl was die Datenübergaben als auch die Datennutzung betrifft. Jedoch stehen die Services auch Firmen, Schulen, NGOs, Journalist_innen und Privatpersonen offen. Soweit möglich, werden Daten zur weiteren freien Verwendung zur Verfügung gestellt.

Besonders prägend war das erste Entwicklungsjahr, in dem viele der grundlegenden Entscheidungen getroffen und die strukturellen Rahmenbedingungen 
für den weiteren Betrieb geschaffen wurden. Darunter fiel auch der Aufbau von drei Gremien, die AUSSDA prägen und unterstützen: Arbeitsgruppe, nationaler Beirat und internationaler Beirat. Die Arbeitsgruppe unterstützt direkt die Leitung von AUSSDA. Sie berät in Fragen der Strategie und zu zukünftigen Entwicklungen. Der nationale Beirat setzt sich derzeit aus Vertreter_innen von 14 Forschungs(förderungs)einrichtungen in ganz Österreich zusammen und unterstützt die Datenakquise und Bekanntmachung von AUSSDA innerhalb und außerhalb der jeweiligen Institutionen. Der internationale Beirat hilft bei der Weiterentwicklung der Serviceangebote, Strukturen und Prozesse von AUSSDA. Dadurch ist das Archiv stärker in die europäische Infrastruktur und in die strategische Weiterentwicklung der internationalen Datenarchivlandschaft eingebunden, als dies allein durch Österreichs Mitgliedschaft in CESSDA ERIC möglich wäre. Eine besondere Herausforderung zu Beginn war die Anstellung von zeitweise bis zu elf Personen, da umfangreiches Handlungswissen in verschiedenen Gebieten für den Betrieb eines digitalen Archivs erforderlich sind. Obwohl AUSSDA sehr von der internationalen Unterstützung anderer Datenarchive profitieren konnte, mussten sämtliche Prozesse für den österreichischen Kontext neu aufgesetzt bzw. entwickelt werden. Neben den Kernprozessen gehörten dazu auch die Entwicklung des prägnanten Markendesigns und einer Präsenz online wie auch offline. So gelang es dann im November 2017, die erste öffentliche AUSSDA-Veranstaltung mit dem Launch des Archivsystems zu feiern. Der Tag, an dem zahlreiche prominente Vertreter_innen von Forschungspolitik und Wissenschaft teilnahmen, fand sehr positiven Widerhall.

Im zweiten Jahr wurden bereits sechsstellige Drittmittelbeträge eingeworben und Vertreter_innen von AUSSDA wurden zu internationalen Veranstaltungen eingeladen, um dort als Best-Practice-Beispiel über den Aufbau der Dateninfrastruktur vorzutragen. Es ergaben sich Gelegenheiten zu internationalen und nationalen Vorträgen, etwa über die "European Open Science Cloud« im Rahmen der österreichischen EU-Ratspräsidentschaft sowie zur Thematik der erforderlichen Maßnahmen und Organisationsentwicklungsziele, die beim Aufbau einer neuen Dateninfrastruktur im ersten Jahr beachtet werden sollten. Letzteres erhielt von mehreren Ländern positive Resonanz, insbesondere von jenen, die derzeit selbst vor der Herausforderung stehen, eine ähnliche Dateninfrastruktur wie AUSSDA einzurichten. Neben solch erfreulichen Highlights bestand die Hauptaufgabe jedoch darin, den Datenbestand wesentlich zu erweitern und viele Arbeitsprozesse in den Regelbetrieb zu überführen. Besonders erfreulich waren die hohen Nutzungszahlen zum Abschluss des zweiten Jahres, die die Erwartungen weit übertrafen.

Die spannende Reise hat gerade erst begonnen und so sollen in den nächsten Jahren weitere Kooperationen etabliert, die bestehenden vertieft und neue Services entwickelt werden. Insbesondere die Möglichkeit eines unmittelbaren Self- 
Deposits wird entwickelt, mit dem Forscher_innen praktisch sofort einen persistenten Identifier für ihre Daten erhalten können. Die Durchführung der eingeworbenen Drittmittelprojekte und die Antragstellung neuer Projekte wird ebenfalls einen spürbaren Anteil neben der täglichen Arbeit haben. Eine besondere Bedeutung wird auch zukünftig die Zusammenarbeit mit den universitären Forschungsservices in Österreich haben. AUSSDA wird gezielt Schulungen zu speziellen Themen anbieten, die üblicherweise nicht Teil der akademischen Ausbildung sind, jedoch zur Erfüllung neuer Anforderungen von Drittmittelgebern, Zeitschriften und der Forschungscommunity gehören. Dies betrifft Themen der Forschungstransparenz, open access, open data und - ganz konkret - Schulungen zur Erstellung von Datenmanagementplänen. Auf internationaler Ebene wird AUSSDA sich auch weiterhin an der Weiterentwicklung der europäischen Standards wie beispielsweise den verwendeten Metadatenschemata und Thesauri beteiligen und die Vernetzung im Rahmen von CESSDAERIC-Projekten fortsetzen. Wesentlich für den bisherigen Erfolg von AUSSDA war die Integration in das stabilisierende Gefüge der Dienstleistungseinrichtung Bibliotheks- und Archivwesen sowie die Anbindung an die beiden weiteren Standorte Graz und Linz mit den beiden Kollegen vor Ort. Hierbei kam besonders der Leiterin der Dienstleistungseinrichtung Bibliotheks- und Archivwesen Maria Seissl eine wichtige Aufgabe zu, indem sie umsichtig die AUSSDA-umgebenden strukturellen Anforderungen berücksichtigte, zugleich jedoch große Freiheit zuließ, was ein kreatives Klima ermöglichte, in dem sich ein Startup wie AUSSDA gut entwickeln ließ und lässt. 
Open-Access-Publikation im Sinne der CC-Lizenz BY-NC-ND 4.0

(c) 2019, Vandenhoeck \& Ruprecht GmbH \& Co. KG, Göttingen ISBN Print: 9783847110989 - ISBN E-Lib: 9783737010986 


\section{Der Umgang mit Objekten im Rahmen von Wissenschaft und Lehre. Die Sammlungskoordinierungsstelle der Universität Wien}

Die Sammlungskoordinierungsstelle wurde im Jahr 2010 an der Dienstleistungseinrichtung Bibliotheks- und Archivwesen eingerichtet und hatte zunächst zum Ziel, sämtliche Objektsammlungen, die zu Lehr- und Forschungszwecken an der Universität Wien angelegt und aufbewahrt wurden, zu dokumentieren sowie Hilfestellungen bei der bestmöglichen Aufbewahrung und dem Erhalt dieser Bestände zu leisten. Seit Einrichtung dieser Stelle wachsen die Aufgabenfelder ständig an, was als Zeichen für einen steten Wandel im Umgang mit Objekten gesehen werden kann.

Der Einrichtung dieser Stelle war ein dreijähriges Projekt vorausgegangen, dessen Aufgabe die Identifizierung und Sichtung der über hundert Sammlungen war und das vom damaligen stellvertretenden Leiter der Dienstleistungseinrichtung Bibliotheks- und Archivwesen Andreas Brandtner geleitet wurde. Zum Zeitpunkt des Projektbeginns war die Situation der Sammlungen an einigen Instituten durchaus prekär: Das Bewusstsein, es mit erhaltungswürdigen Beständen zu tun $\mathrm{zu}$ haben, war vielerorts nicht vorhanden. Die Bezeichnung "Sammlung" für in Kartons verpackte, vom Staub der Jahrzehnte bedeckte Objekte schien für einzelne Kolleg_innen maßlos übertrieben. Hier galt es zunächst das Bewusstsein für die Besonderheit und Einzigartigkeit dieser Bestände zu bilden und Rahmenbedingungen zu schaffen, die einen langfristigen Erhalt dieser Sammlungen ermöglichten. Schon dem Projekt wurden finanzielle Mittel zur Verfügung gestellt, um bestandserhaltende Maßnahmen wie Restaurierungen und Digitalisierungen durchführen zu können. Der Koordinierungsstelle steht seit ihrem Beginn weiterhin jährlich ein Budget zur Verfügung, das für bestandserhaltende Maßnahmen zweckgebunden ist. Die finanzielle Ausstattung und die Anbindung der Koordinierungsstelle an die Dienstleistungseinrichtung Bibliotheks- und Archivwesen zeichnen diese Stelle im deutschsprachigen Raum aus und machen sie unter der Bezeichnung »Wiener Modell« zu einem Best-Practice-Modell. Dass die Koordinierungsstelle sich so gut und beispielhaft entwickeln konnte, ist der Leiterin der Dienstleistungseinrichtung Bibliotheks- und Archivwesen Maria Seissl zu verdanken, die das Potential und 
die Notwendigkeit einer solchen Stelle erkannt und von Beginn an bestmöglich unterstützt hat.

Denn heute, zwölf Jahre nach Start des Projekts, nach Erlass einer Sammlungsordnung und -strategie durch das Rektorat der Universität Wien im Jahr $2013^{1}$ und eines regen Netzwerks, dank dem nicht nur ein Buch ${ }^{2}$, sondern auch eine Ausstellung im Naturhistorischen Museum ${ }^{3}$ gemeinsam gestaltet werden konnte, hat sich die Situation für die Sammlungen insgesamt deutlich verbessert. Das ist nicht zuletzt an jenen Themen zu sehen, die aktuell im Bereich Sammlungen diskutiert werden, nämlich die Frage nach Provenienz, Herkunftsgeschichten und Entstehungskontexten der Objekte. Seit der Entdeckung eines Eintrags in einem Inventarbuch, in dem die Übernahme mehrerer Objekte von der Gestapo im Jahr 1938 verzeichnet war, arbeitet die Sammlungskoordinierungsstelle auch eng mit dem Arbeitsbereich NS-Provenienzforschung an der Universitätsbibliothek Wien ${ }^{4}$ zusammen. Aufgrund der überwältigenden Menge an Sammlungsobjekten und dem sehr unterschiedlichen Erschließungsgrad der Bestände (manche Objekte sind einzeln inventarisiert, andere Sammlungen sind noch nicht einmal grob erfasst) ist eine systematische Durchsicht der Sammlungsbestände hinsichtlich unrechtmäßiger Erwerbungen - nicht nur im NSKontext - praktisch unmöglich. Umso wichtiger ist es, die Sammlungsmitarbeiter_innen für diese Problematik zu sensibilisieren, um so überhaupt $\mathrm{zu}$ Verdachtsfällen zu kommen, denen dann kritisch nachgegangen werden kann.

Ein Ahnenschädel aus Papua-Neuguinea, der sich in der Ethnografischen Sammlung der Universität Wien befindet, zeigt die aktuellen Themenfelder sehr deutlich und ist in vielerlei Hinsicht typisch für Objekte aus den Lehr- und Forschungssammlungen der Universität Wien. Typisch ist zum einen die ungewisse Provenienz, die im Falle von menschlichen Überresten besonders problematisch ist. Zum anderen war die Art und Weise, wie dieser Schädel die längste Zeit in der Sammlung aufbewahrt wurde, alles andere als angemessen: Als Anschauungsobjekt für die Lehre lag er in einer Vitrine zwischen anderen Objekten aus der Region Ozeanien bereit, um für entsprechende Lehrveranstaltungen schnell auffindbar und griffbereit zu sein. Heute wird der Schädel in einem verschlossenen Stahlschrank gelagert, um ihn neugierigen Blicken zu

1 In: Mitteilungsblatt der Universität Wien vom 01.03.2013, 16. Stück, Nr. 95.

2 Schaukästen der Wissenschaft. Die Sammlungen an der Universität Wien. Hg. von Claudia Feigl. Wien u. a.: Böhlau 2012. Der Band erschien 2016 unter dem Titel »Academic Showcases. The Collection at the University of Vienna " auch in englischer Sprache.

3 Die Ausstellung Das Wissen der Dinge wurde anlässlich des 650jährigen Jubiläums der Universität Wien gezeigt und war vom 6. Mai 2015 bis 10. Jänner 2016 im Saal 50 des Naturhistorischen Museums Wien zu sehen.

4 Siehe Beitrag von Markus Stumpf über den Arbeitsbereich NS-Provenienzforschung in diesem Band. 
entziehen und unvorbereitete Begegnungen zu vermeiden. Und erst vor kurzem wurden dringend notwendige Konservierungsmaßnahmen sorgsam durchgeführt.

Die frühere Art der Aufbewahrung verweist auch auf ein weiteres typisches Merkmal, das auf sehr viele Universitätssammlungen zutrifft: die rasche Historisierung der Objekte. Denn mit dem rasanten Wandel der didaktischen Methoden und vor allem der Entwicklung der neuen Medien, rückten die Lehrsammlungen rasch in den Hintergrund, wurden in Kisten verpackt und vergessen. Oder, wie im Falle der Ethnografischen Sammlung: Die Sammlung wanderte samt Vitrinen in den Keller, und viele der einst intensiv genutzten Sammlungen wurden so zu historischen Beständen. Genau diese Sammlungen werden nun verstärkt aus ihrem Dornröschenschlaf geweckt und im Zuge von Projekten bearbeitet: Seien es die höchst bedenklichen Erwerbungen, die in den ersten Jahren nach der Gründung des Instituts für Theater-, Film- und Medienwissenschaft im Jahr 1942 getätigt wurden, seien es die Sammlungen am Department für Anthropologie oder die oben erwähnte Ethnographische Sammlung - Provenienz und Herkunftsgeschichten der Sammlungsobjekte rücken hier in den Mittelpunkt des Forschungsinteresses und bringen interessante Erkenntnisse zur Institutsgeschichte und den beteiligten Akteur_innen. In diese Projekte wird die Koordinierungsstelle gerne eingebunden, um eine Vernetzung mit anderen Sammlungen zu forcieren bzw. umgekehrt, andere Sammlungen an den eigenen Überlegungen und Ergebnissen teilhaben zu lassen. Mitgedacht wird dabei immer auch der Aspekt Digitalisierung und die Frage der Zugänglichkeit der Daten für eine interessierte Öffentlichkeit. Die Frage nach den Eigentumsverhältnissen der Objekte, nach den Nutzungsrechten an deren Bildern sowie ethische Gesichtspunkte spielen dabei eine große Rolle, und nicht immer kann dem Wunsch nach größtmöglicher Öffnung der Bestände (zumindest im digitalen Raum) Folge geleistet werden.

Dass das Interesse an Objekten aus den Sammlungen groß ist, beweisen nicht zuletzt auch die vielen Leihansuchen, die die Koordinierungsstelle und die Sammlungsmitarbeiter_innen immer häufiger erreichen. Zur Zeit des Abfassens dieses Artikels war die Universität Wien in knapp zehn internationalen Ausstellungen mit Sammlungsobjekten vertreten, u. a. im Naturhistorischen $\mathrm{Mu}-$ seum Wien, im Maximilianmuseum Augsburg und im Deutschen Hygienemuseum Dresden. Primäres Ziel der Koordinierungsstelle ist es jedoch, die Sammlungsbestände bestmöglich für ihre eigentlichen Zwecke, nämlich für Lehre, Wissenschaft und Forschung, nutzbar zu erhalten bzw. nutzbar zu machen - und das nicht nur für unsere, sondern auch für zukünftige Generationen. 
Open-Access-Publikation im Sinne der CC-Lizenz BY-NC-ND 4.0

(c) 2019, Vandenhoeck \& Ruprecht GmbH \& Co. KG, Göttingen ISBN Print: 9783847110989 - ISBN E-Lib: 9783737010986 


\section{Guido Blechl}

\section{Open Access: Von den Anfängen bis heute}

Open Access war Mitte der 2000er Jahre an der Universitätsbibliothek Wien noch kein großes Thema. Eine einschlägige Podiumsdiskussion, zu der auch die Universitätsbibliothek eingeladen war, musste etwa mangels Interesse von Seiten der wissenschaftlichen Community kurzfristig abgesagt werden. Denn die Zeit für Open Access war noch nicht reif.

Nichtdestotrotz tauchte in den Folgejahren das Thema immer häufiger auf, sodass Maria Seissl ab 2008 eine Open-Access-Arbeitsgruppe einsetzte. Ziel war es, das Thema Open Access systematisch aufzurollen, Chancen und Herausforderungen für die Universitätsbibliothek und Universität Wien auszuloten und gegebenenfalls auch entsprechende Services zu entwickeln. Viele Sitzungen später konnten erste Erfolge präsentiert werden. Die Open-Access-Website der Universität Wien ${ }^{1}$ ging im Jahr 2009 online, und 2010 unterzeichnete das Rektorat die »Berliner Erklärung«. Dies war ein wichtiges Signal für alle Forschenden, denn es bedeutete: Die Universität Wien unterstützt von jetzt an offiziell Open Access. Im selben Jahr fand im Kleinen Festsaal der Open-Access-Informationstag statt, zu dem sich bereits rund hundert Interessierte einfanden. ${ }^{2}$

Von da an ging es in großen Schritten weiter: 2012 ging das institutionelle Repositorium u:scholar online, sodass wissenschaftliche Literatur der Universität Wien von nun an in dieser PHAIDRA-Collection systematisch gesammelt und weltweit frei zugänglich angeboten werden konnte. Und erstmals fand in Österreich ein großes Open-Access-Event statt: Mehr als 200 Besucher_innen aus Österreich, Deutschland und der Schweiz nahmen an den »Open-AccessTagen«, der größten einschlägigen Konferenz zum Thema im D-A-CH-Raum, teil.

$1 \mathrm{https}: / /$ openaccess.univie.ac.at/.

2 Welche Open-Access-Themen bei der abschließenden Podiumsdiskussion von Maria Seissl und den anderen Teilnehmer_innen damals diskutiert wurden, kann man in der Audioaufzeichnung zum Thema »Open Access - quo vadis Austria?» im frei zugänglichen Repositorium PHAIDRA nachhören: https://phaidra.univie.ac.at/o:51440. 
Schließlich war auch die Zeit reif, um ein permanentes Service für Open Access anzubieten. Das »Open Access Office« wurde 2013 an der Universitätsbibliothek eingerichtet, um Angehörige der Universität Wien bei allen ihren Anliegen rund um das Thema Open Access zu unterstützen. Als Open-AccessInfrastruktur konnte neben u:scholar bereits das System OJS angeboten werden, eine zentrale Softwareinstallation, welche Universitätsangehörige für die Herausgabe von eigenen Open-Access-Zeitschriften nutzen können.

Noch aber fehlte ein wichtiger Baustein, um ein wirklich vollständiges Service-Portfolio für Open Access anbieten zu können: Ein Open-Access-Publikationsfonds, der es Forschenden ermöglicht, in kostenpflichtigen Open-AccessJournals zu publizieren. Dies war wohl einer der schwierigeren Schritte beim Ausbau des Serviceangebotes, da hier seitens des Rektorats zusätzliches Budget bereitgestellt werden musste. Um hier fundierte Entscheidungen treffen zu können, wurde für das Rektorat eine eigene Studie mit dem Titel Der Goldene Weg des Open Access zum funktionalen Publikationswesen. Handlungsoptionen für die Universität Wien erstellt. ${ }^{3}$

Schließlich war es dann soweit: Nach einer intensiven Diskussion zur strategischen Entwicklung von Open Access an der Universität Wien - diese fand in einem dafür eigens eingerichteten Sounding Board mit Forschenden und Stakeholdern der Universität statt - konnte am 17. Juni 2014 nicht nur der OpenAccess-Publikationsfonds, sondern gleichzeitig auch die Open-Access-Policy der Universität Wien präsentiert werden.

Damit waren alle Voraussetzungen für eine positive Entwicklung von Open Access vorhanden. In den Folgejahren nahm das Interesse der Forschenden an Open Access kontinuierlich zu. Maßgeblich dazu beigetragen haben auch die ab 2015 von der Clearingstelle Konsortien der Universitätsbibliothek Wien und der Kooperation E-Medien Österreich (KEMÖ) verhandelten Konsortialverträge mit Open-Access-Komponente, die es Universitätsangehörigen ermöglichte, ihre Artikel ohne Zusatzkosten in renommierten Journals der großen Wissenschaftsverlage open access zu publizieren.

Neben diesen lokalen Aktivitäten konnte die Bedeutung von Open Access durch Teilnahme der Universitätsbibliothek an zahlreichen nationalen und internationalen Kooperationen und Initiativen weiter ausgebaut werden, etwa der Teilnahme am EU-Projekt OpenAIRE ${ }^{4}$ ab 2010 (inkl. der Folgeprojekte), der Mitwirkung am Open Access Network Austria (OANA) ${ }^{5}$ ab 2012, der Mitwirkung

3 Volltext verfügbar unter http://phaidra.univie.ac.at/o:337723.

4 Open Access Infrastructure for Research in Europe, siehe für weiterführende Informationen https://www.openaire.eu/ [Letzter Zugriff: 11.09.2019].

5 Open Science Network Austria (vormals Open Access Network Austria), siehe für weiterführende Informationen https://www.oana.at/ [Letzter Zugriff: 11.09.2019]. 
an der SCOAP3-Initiative ${ }^{6}$ ab 2014, der Lead am HochschulraumstrukturmittelProjekt "Austrian Transition to Open Access (AT2OA) « ab 2017 oder auch der Support der Initiativen $\operatorname{arXiv}^{8}, \mathrm{COAR}^{9}, \mathrm{OAPEN}^{10}$, Knowledge Unlatched ${ }^{11}$ und Open Library of Humanities $(\mathrm{OLH})^{12}$.

Maria Seissl hat viele der hier genannten Punkte nicht nur strategisch mitentwickelt, sondern sie übernahm in vielen Fällen auch leitende und operative Funktionen, etwa als aktive Teilnehmerin der AG Open Access in der Anfangsphase oder später als Projektleiterin von AT2OA.

Noch ist die Open-Access-Geschichte der Universitätsbibliothek Wien nicht abgeschlossen - einige Kapitel müssen wohl noch geschrieben werden, bis die sogenannte Transformationsphase ein Ende findet. Aufgrund der bisherigen Erfahrungen kann man in jedem Fall davon ausgehen, dass die Universitätsbibliothek auch bei den künftigen Entwicklungen ganz vorne dabei sein wird.

6 Sponsoring Consortium for Open Access Publishing in Particle Physics, siehe für weiterführende Informationen https://scoap3.org/ [Letzter Zugriff: 11.09.2019].

7 Austrian Transition to Open Access (AT2OA), siehe für weiterführende Informationen https://www.at2oa.at/ [Letzter Zugriff: 11.09.2019].

8 ArXiv.org, siehe für weiterführende Informationen https://arxiv.org/ [Letzter Zugriff: 11.09. 2019].

9 Confederation of Open Access Repositories, siehe für weiterführende Informationen https:// www.coar-repositories.org/ [Letzter Zugriff: 11.09.2019].

10 Open Access Publishing in European Networks, siehe für weiterführende Informationen https://www.oapen.org/ [Letzter Zugriff: 11.09.2019].

11 Knowledge Unlatched, siehe für weiterführende Informationen http://www.knowledgeun latched.org/ [Letzter Zugriff: 11.09.2019].

12 Open Library of Humanities, siehe für weiterführende Informationen https://www.openlibh ums.org/ [Letzter Zugriff: 11.09.2019]. 
Open-Access-Publikation im Sinne der CC-Lizenz BY-NC-ND 4.0

(c) 2019, Vandenhoeck \& Ruprecht GmbH \& Co. KG, Göttingen ISBN Print: 9783847110989 - ISBN E-Lib: 9783737010986 


\section{»Druckt ihr eh alles aus was in PHAIDRA ist?《 Die Abteilung Repositorienmanagement PHAIDRA-Services - ein Paradigmenwechsel}

Als 2007 das Projekt DAMS (Digital Asset Management System) gestartet wurde, war kaum abzusehen, welch zahlreiche neuen Aufgabenbereiche sich für Bibliotheken im Bereich der langfristigen Archivierung von digitalen Objekten entwickeln würden. Der Begriff »Repositorium « war noch neu und das Vertrauen in die langfristige Auffindbarkeit von Inhalten im Internet gering, wie die eingangs zitierte Frage, die kurz nach Projektstart an uns gestellt wurde, zeigt. Das erste Jahr wurde intensiv dazu genutzt, von einigen ausgewählten Pilotpartnern - Forschenden, Lehrenden und Sammlungsverantwortlichen an der Universität Wien - zu erfahren, wie ein digitales Archivierungssystem aussehen könnte. Damit begann eine bis heute spannend gebliebene Reise durch unterschiedliche Fachgebiete.

Seit Projektbeginn besteht eine enge Zusammenarbeit mit dem Zentralen Informatikdienst, in der beide Teams viel voneinander und vor allem miteinander lernen. Stück für Stück entwickelte sich so über die Jahre eine gemeinsame Expertise, die ausschlaggebend für den Erfolg war und ist.

Als genau ein Jahr nach Projektbeginn PHAIDRA (Permanent Hosting and Archiving of Digital Resources and Assets) online zur Verfügung stand, lag der Fokus auf der Archivierung einzelner digitaler Ressourcen. Das erste in PHAIDRA archivierte Objekt war die Ausgabe 08/1 von comment, der Zeitschrift des Zentralen Informatikdienstes. Darin schrieb der damalige technische Leiter von PHAIDRA: "Auch das schönste Digital Asset Management System ist nur ein Mittel zum Zweck - der eigentliche Zweck sind die Inhalte. Wir hoffen, dass der Reichtum der Universität Wien an interessanten Sammlungen, Lernobjekten und aktuellen Forschungsergebnissen bald durch Phaidra für die Zukunft gesichert und der Weltöffentlichkeit präsentiert wird.»" Diese Hoffnung hat sich mittlerweile erfüllt.

1 Peter Marksteiner: PHAIDRA. Eine Plattform für hochwertige digitale Inhalte. In: comment 08/1, S. 19-24, https://phaidra.univie.ac.at/o:1. 
Es begann eine intensive Zeit der Bewerbung von PHAIDRA im Rahmen von Vorträgen, Workshops und Publikationen und mit dem Knüpfen eines großen Netzwerkes. Bald meldeten sich Vertreter_innen von anderen Universitäten um zu erfahren, ob und wie man mit uns kooperieren könnte. Damit startete eine erfolgreiche internationale Zusammenarbeit mit anderen Institutionen, die mittlerweile 20 Partner umfasst. ${ }^{2}$ Da PHAIDRA auf der OpenSource-Software Fedora aufgebaut wurde, kann es den Bedürfnissen der Nutzer_innen folgend, ständig angepasst werden, beispielsweise mit Möglichkeiten des Massenuploads, also des Hochladens mehrerer Objekte gleichzeitig. Internationale Kontakte, wie etwa zu COAR (Confederation of Open Access Repositories), ließen das Wissen ebenso wachsen wie die guten internen Kontakte zu anderen Abteilungen der Universitätsbibliothek Wien, etwa zur Forschungsdokumentation u:cris oder zur Abteilung »Alte und wertvolle Bestände«. Historisch wertvolle Bücher werden an der Universitätsbibliothek Wien gescannt, mit Metadaten versehen und mittels eines immer stärker automatisierten Workflows in PHAIDRA hochgeladen und im Bookviewer dargestellt. Ein wichtiger Meilenstein war die Etablierung des Institutional Repositories u:scholar, eines eigenen Bereichs von PHAIDRA, dessen Oberfläche zum Hochladen von Artikeln den Bedürfnissen der Forschenden angepasst wurde. Wissenschaftlicher Output der Universität Wien kann so sicher in PHAIDRA archiviert werden. Auch die Eingliederung des Digital Asset Management Systems UNIDAM in die Abteilung stellte eine weitere Bereicherung dar. Damit werden Lehrenden einige Services geboten, wie etwa eine flexible Vergabe von Metadaten und unterschiedliche Visualisierungsmöglichkeiten digitaler Objekte. Mit den Anforderungen jedes neuen Forschungsprojekts wuchsen die Kompetenzen, zum Beispiel bzgl. des Umgangs mit Karten während des Projekts »UB-Maps«. Die Anbindung an das Projekt »Open Education Austria ${ }^{3}$ führte zu einer Weiterentwicklung von PHAIDRA hinsichtlich offener Lehr- und Lernressourcen. Das Projekt »Ethnographische Datenarchivierung " ${ }^{4}$, bei dem auch sensible und zum Teil sehr komplexe Daten archiviert werden sollen, stieß die Entwicklung eines neuen Objekttyps an.

Die international immer stärkere Forderung nach Datenmanagementplänen fand ebenso Niederschlag in der Abteilung wie die Nachfrage nach Digital Object Identifiern (DOI). ORCIDs, permanente Identifikatoren für Forschende, können ebenfalls in PHAIDRA hinterlegt werden. Die Abteilung »Repositorienmanagement PHAIDRA-Services« sieht sich gemeinsam mit dem Team »Software Development « am Zentralen Informatikdienst auch als Beratungsstelle für Forschende hinsichtlich ihres nachhaltigen und ethischen Kriterien entsprechenden

2 Siehe https://www.phaidra.org/community/phaidra-partners/ [Letzter Zugriff: 11.09.2019].

3 https://www.openeducation.at/home/ [Letzter Zugriff: 11.09.2019].

4 https://eda.univie.ac.at/ [Letzter Zugriff: 11.09.2019]. 
Umgangs mit Daten. Diesbezüglich ist strukturell noch einiges zu tun, denkt man etwa an das Konzept von Data Stewards ${ }^{5}$, bei dem Expert_innen aus unterschiedlichen Fachbereichen sich des Forschungsdatenmanagements annehmen.

PHAIDRA war und ist immer wieder in nationale und internationale Projekte involviert. TEMPUS (Trans-European Mobility Programme for University Studies) führte zum Einsatz von PHAIDRA in Ländern des Balkans wie Serbien, Bosnien und Herzegowina sowie Montenegro, und Europeana Libraries bedeutete einen großen Zugewinn an digitalisierten Nachlässen, Bildern, Sammlungen und Büchern in PHAIDRA. Mit OpenAIRE (Open Access Infrastructure for Research in Europe $)^{6}$ wurden bereits zahlreiche gemeinsame Veranstaltungen durchgeführt. Das Projekt e-Infrastructures Austria, ein vom österreichischen Bundesministerium für Bildung, Wissenschaft und Forschung gefördertes Projekt von neun österreichischen Universitäten ${ }^{7}$, war ein wichtiger Impulsgeber, denn hier konnte Kompetenz in unterschiedlichen Bereichen ausgebaut und zugleich weitergegeben werden. Ein Wissensaustausch findet auch in diversen Weiterbildungskursen, wie dem Universitätslehrgang Library and Information Studies oder dem neu etablierten Lehrgang "Data Librarian «, in dem Kenntnisse, die für Entwicklung und Umsetzung von Services im Bereich Forschungsdatenmanagement von Nutzen sind, vermittelt werden, statt. 2016 wurde das Netzwerk für Repositorienmanager_innen ${ }^{8}$ gegründet, da mittlerweile die Handlungsfelder und Aufgabenbereiche rund um Repositorien so angewachsen waren, dass eine enge Zusammenarbeit mit anderen Institutionen angebracht schien. Das sogenannte »RepManNet« ist ein Zusammenschluss von Verantwortlichen aus Institutionen in ganz Österreich, die ein Repositorium betreuen und sich mit anderen Kolleg_innen über Erfahrungen, Herausforderungen und zukünftige Themen austauschen möchten. Es ist beim Forum der Universitätsbibliotheken Österreichs (ubifo) ${ }^{9}$ angesiedelt und umfasst bereits knapp 90 Personen aus 38 Institutionen.

Sich immer wieder zu fragen, was die Nutzer_innen des Systems heute und in Zukunft benötigen, und dabei die rasant wachsenden technischen Möglichkeiten im Blick zu behalten, ist eine ständige Herausforderung. Dafür ist es unerlässlich, grenzüberschreitend stets in Kontakt mit anderen Universitäten zu

5 Siehe das Beispiel der Universität Delft: https://www.tudelft.nl/en/library/current-topics/re search-data-management/research-data-management/data-stewardship/ [Letzter Zugriff: 11.09.2019].

$6 \mathrm{https}: / /$ www.openaire.eu/ [Letzter Zugriff: 11.09.2019].

7 https://www.e-infrastructures.at/de/ [Letzter Zugriff: 11.09.2019].

$8 \mathrm{https} / / /$ datamanagement.univie.ac.at/rdm/netzwerk-fuer-repositorienmanagerinnen-repm annet/ [Letzter Zugriff: 11.09.2019].

9 https://ubifo.wordpress.com/ziele/ [Letzter Zugriff: 11.09.2019]. 
bleiben und sich nicht zuletzt mit aktuellen Trends und Vorgaben auf EU-Ebene vertraut zu machen, wie beispielsweise mit der EOSC (European Open Science

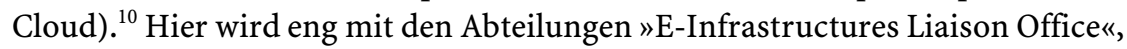
in dem unter anderem EOSC-bezogene Agenden übernommen werden, und AUSSDA (The Austrian Social Science Data Archive) ${ }^{11}$ zusammengearbeitet. Die nächsten Jahre werden spannend bleiben: Die Hochschulschriften der Universität Wien werden nach PHAIDRA migriert, es wird in PHAIDRA eine temporäre Archivierung ermöglicht und der Umstieg auf FEDORA 6 erfordert großes technisches Knowhow.

In einem innovativen, sich ständig ändernden Bereich zu arbeiten, bedeutet stets, das Herkömmliche nicht aus den Augen zu verlieren und gleichzeitig mutig Neues auszuprobieren. Diese perfekten Rahmenbedingungen für die Etablierung und den laufenden Betrieb des universitären Repositoriums waren und sind dank der Leitung der Universitätsbibliothek Wien gegeben - dafür großen Dank an Maria Seissl.

10 https://www.eosc-portal.eu/ [Letzter Zugriff: 11.09.2019].

11 Siehe https://aussda.at/ [Letzter Zugriff: 11.09.2019] sowie den Beitrag von Lars Kaczmirek in diesem Band. 


\section{Der ganz normale Wahnsinn und manchmal sogar ein Krimi. ubw:helpdesk und User Training}

Wenn man viel mit Menschen zu tun hat, sind die Zeiten und Situationen immer bewegt und manchmal auch bewegend. Das wusste auch Maria Seissl, als sie dieser kleinen und feinen Abteilung, die es seit 2001 gibt und die heute oft noch immer nach der ursprünglichen Bezeichnung »das Zer (Zentrum für elektronische Recherchen) oder - in Unkenntnis der Bedeutung - »der Zer « genannt wird, die Aufgabe anvertraute, alle E-Mails und Telefonate an zentraler Stelle zu beantworten. Der Diversität der Anfragen und der Breite des Aufgabenspektrums, die um ein reiches Schulungsangebot erweitert wurde, Rechnung tragend, wurde die Abteilung im Jahr 2013 in ubw:helpdesk und User Training umbenannt. Der Helpdesk war und ist neben dem Infopult vor dem Großen Lesesaal der Hauptbibliothek mit allen nur denkbaren Fragestellungen per Telefon und E-Mail konfrontiert. In der Regel sind es Fragestellungen, die mit unserem Bibliotheksangebot zu tun haben, manchmal aber geht es auch weit darüber hinaus. Etwa, als uns ein selbsternannter Krimiautor unzählige Mappen mit Kopien von Polizeiakten zusandte, nachdem er sich zuvor per E-Mail nicht abweisen hatte lassen, uns seinen Krimi, der dann eben unerwartet aus diesen Mappen bestand, als Geschenk zukommen zu lassen. Über diesen und ähnlich kuriose Fälle wird die Direktion natürlich in Kenntnis gesetzt.

Unter Maria Seissl entwickelte sich auch das Thema Teaching Library, zu dem unsere Abteilung wesentliche Beiträge leistet. Nachdem die Ära der alten IVS (Informationsvermittlungsstelle) zu Ende gegangen war, begannen wir mit regelmäßigen Schulungsangeboten. Im Lauf der Zeit wurden Schulungen rund um die Benutzung unserer elektronischen Ressourcen immer wichtiger und sind heute ein Charakteristikum unserer Bibliothek. In die Zeit Maria Seissls fällt die generelle Entwicklung von gedruckten zu elektronischen Ressourcen. In unserer Bibliothek hieß das gleichzeitig weg vom traditionellen (Online-)Katalog hin zur Suchmaschinentechnologie, die hier unter der Bezeichnung "u:search " den OPAC ersetzte. Maria Seissl verfolgt das strategische Ziel, bei diesen Entwicklungen die Universitätsbibliothek Wien als Early Adopter zu positionieren. Daher waren und sind wir bei vielen Umstellungen oft unter den Ersten, was für 
unsere Abteilung heißt, die mit Neuerungen einhergehenden flankierenden Schulungsmaßnahmen effizient zu erarbeiten und effektiv umzusetzen. Dass unserer Abteilung dies gelingt, hat uns Maria Seissl stets zugetraut, so auch den Umstieg auf ein Ticketsystem für die Anfragebeantwortung mit einem integrierten Ideenforum. Dass wir selbst am besten wüssten, welches Schulungsangebot wir anbieten, dass wir in allen Situationen die richtigen Worte mit den Benutzer_innen fänden, für all dies gab uns Maria Seissl immer freie Hand. Unsere Abteilung konnte und kann unter diesen Voraussetzungen auch optimal für ganz spezielle Wünsche der Benutzer_innen da sein, sei es eine Einzelrecherche für ein Dissertations- oder Diplomarbeitsthema, sei es eine komplexe Suche nach einem Artikel oder einem Buch, sei es, dass Benutzer_innen bei der Benutzung der elektronischen Ressourcen Schwierigkeiten haben. Zu den Voraussetzungen zählt gleichzeitig die besonders gute Zusammenarbeit mit dem Team E-Resource Management, dem Infodienst und der Entlehnung der Hauptbibliothek. Besonders stolz sind wir auf die große Zahl von Studienanfänger_innen, die wir jedes Semester in die Benutzung der Bibliothek einführen, beim Betreuten Recherchieren begleiten oder einfach die Nutzung von E-Ressourcen nahebringen, sowie auf Spezialschulungen, die wir gemeinsam mit anderen kompetenten Kolleg_innen für Dissertierende anbieten können. Eine spezielle Zusammenarbeit gibt es seit einigen Semestern mit dem Center for Teaching and Learning im Rahmen der Ausbildung von Schreibmentor_innen, wo wir unsere Kompetenzen regelmäßig in Workshops einbringen. Zusammen mit der Arbeitsgruppe Teaching Library der Universitätsbibliothek werden auch regelmäßig sogenannte TeachMeets veranstaltet, die neben Personen aus dem bibliothekarischen Umfeld auch Lehrende der Universität Wien ansprechen. Maria Seissl hat uns stets unterstützt und ermutigt, solche Veranstaltungen durchzuführen. Als sie das jüngste TeachMeet persönlich eröffnete, war sie selbst positiv überrascht, wie viele Personen der Universität teilgenommen haben. Mit viel Geduld hat sie auch die langsame Entstehung und Erweiterung des OnlineTutorials zur Vermittlung von Recherche- und Informationskompetenz für Studierende auf Moodle gefördert, an dem die Abteilung im Rahmen der Teaching Library beteiligt ist.

In den nächsten Jahren warten noch viele Herausforderungen auf die Abteilung ubw:helpdesk und User Training. Ein möglicher Umbau der Hauptbibliothek würde die Umstellung des Schulungsangebotes auf eine rein elektronische Ebene beschleunigen und bedeutet, dass rasch eine Reihe von Tutorials für die Benutzer_innen erstellt werden müssen. Jede einzelne Änderung, die in dieser Bibliothek gemacht wird und die sich auf die Benutzer_innen auswirkt, erhöht das Anfrageaufkommen und ändert die Fragestellungen der Benutzer_innen und betrifft damit die Abteilung ubw:helpdesk und User Training unmittelbar. Bei 
uns heißt es daher dranbleiben und in bewährter Weise gemeinsam mit der Leiterin die Herausforderungen der nächsten Jahre meistern. 
Open-Access-Publikation im Sinne der CC-Lizenz BY-NC-ND 4.0

(c) 2019, Vandenhoeck \& Ruprecht GmbH \& Co. KG, Göttingen ISBN Print: 9783847110989 - ISBN E-Lib: 9783737010986 


\section{»Ihr seid's ja nicht von der UB, ihr seid's von der Uni!« Institutioneller Wandel illustriert anhand der Einführung des Forschungsinformationssystems u:cris}

Seit 2013 ist an der Universität Wien das Forschungsinformationssystem u:cris ${ }^{1}$ in Betrieb. Es verzeichnet Publikationen, Drittmittelprojekte, Vorträge, Auszeichnungen und andere Aktivitäten ihrer Wissenschafter_innen. Einerseits sind diese Informationen für das interne und externe Berichtswesen von Relevanz, andererseits kann sich die interessierte Öffentlichkeit inner- und außerhalb der Universität Wien über das öffentliche u:cris-Portal ${ }^{2}$ einen Überblick über die vielfältigen Forschungsaktivitäten der Wissenschafter_innen der Universität Wien verschaffen. Hinter dem Namen u:cris verbirgt sich zum einen die Standardsoftware PURE von Elsevier, die heute an mehr als 250 Universitäten und Forschungseinrichtungen weltweit in Verwendung ist, zum anderen auch ein Team der Universitätsbibliothek Wien, das derzeit aus fünf Personen besteht.

\section{Von den Anfängen der Forschungsdokumentation zur Research Activities Documentation (RAD)}

Seit 2004 sind die Agenden der Forschungsdokumentation an der Universitätsbibliothek Wien verankert. u:cris reiht sich als mittlerweile viertes System zur Dokumentation von Forschungsleistungen an der Universität Wien ein. An $\mathrm{u}$ :cris und seinen Vorgängersystemen lassen sich sehr gut die bedeutsamsten Veränderungen des Bereichs Forschungsinformationsmanagement von seinen Anfängen bis hin zu einer elaborierten digitalen Forschungsberichterstattung ablesen.

Es lohnt sich, einen historischen Blick auf die Vorgängersysteme von u:cris zu werfen: Von den frühen 1990er Jahren bis 2000 war das System DOnkey

\footnotetext{
1 CRIS: Das Akronym steht für Current Research Information System, einer international üblichen Bezeichnung für Forschungsinformationssystem. Das u: ist das Branding der Universität für digitale Services.

2 https://ucris.univie.ac.at/.
} 
(Documentation on keypress) in Betrieb. Danach wurde die sogenannte "Salzburger Datenbank «, die zum damaligen Zeitpunkt als State-of-the-art-Lösung in Österreich galt, auch an der Universität Wien eingeführt. 2004 wurde diese samt Personal gleichsam in einer »Nacht-und-Nebel-Aktion " (aus der Perspektive des damals betroffenen Mitarbeiters mit 15 Jahren Zeitabstand) aus der damals existierenden Organisationseinheit für "Qualitätsmanagement und Drittmittelförderung « an die Universitätsbibliothek Wien transferiert. 2005 begannen erneut Vorarbeiten für die Einführung einer neuen Forschungsdokumentation, die während ihres Betriebs von 2006 bis 2013 unter dem Namen Research Activities Documentation (RAD) Bekanntheit erlangte. 2013 wurde die Standardsoftware PURE an der Universität Wien nach einjähriger Implementierungszeit unter dem Namen u:cris in Betrieb genommen. Auf RAD und u:cris lohnt es sich im Folgenden, einen näheren Blick zu werfen, da sich die beiden Systeme von ihrem Grundkonzept her gleichsam als Gegenpole gegenüberstehen: auf der einen Seite ein an der Institution selbstentwickeltes, individuell maßgeschneidertes System, auf der anderen ein Standardsystem eines kommerziellen Anbieters.

\section{Research Activities Documentation (RAD)}

Aufgrund neuer gesetzlicher Vorgaben (UG 2002 ${ }^{3}$, Wissensbilanz-Verordnung $2006^{4}$ ) wurden die österreichischen Universitäten verpflichtet, ab dem Berichtsjahr 2006 Rechenschaft über eine Reihe von Kennzahlen abzulegen. Dies betraf neben vielen Daten, die aus der täglichen Verwaltung erwuchsen, auch Informationen über Publikationen, Vorträge, Aktivitäten in der wissenschaftlichen Community oder Aufenthalte von Gastwissenschafter_innen. Zu diesem speziellen Zweck wurde damals an der Universität Wien beschlossen, innerhalb des Campusmanagementsystems i3v ein eigenes Modul für die Forschungsberichterstattung mit dem Namen Research Activities Documentation (RAD) aus der Taufe zu heben. Das RAD-Projekt war eine Kooperation der Dienstleistungseinrichtungen Forschungsservice und Internationale Beziehungen (wo auch die Projektleitung verortet war), Zentraler Informatikdienst und Universitätsbibliothek Wien. Mit an Bord war damals auch noch die Medizinische Universität Wien. Entwickelt wurde hausintern, also am Zentralen Informatikdienst, wo die entsprechenden Ressourcen und Kapazitäten vorhanden waren,

3 https://www.ris.bka.gv.at/GeltendeFassung.wxe? Abfrage=Bundesnormen \& Gesetzesnummer $=20002128$ [Letzter Zugriff: 27.08.2019].

4 https://www.ris.bka.gv.at/GeltendeFassung.wxe?Abfrage $=$ Bundesnormen $\&$ Gesetzesnummer $=20009519$ [Letzter Zugriff: 27.08.2019]. 
und für den damaligen Stand der Technik entsprach diese hochintegrierte Lösung den damaligen Regeln der Kunst. Insbesondere konnte zum damaligen Zeitpunkt kein Produkt auf dem Markt den Anspruch erheben, diese komplexen Anforderungen zu erfüllen.

Die Inbetriebnahme erfolgte unter großem Zeitdruck und aus heutiger Sicht war vielleicht zu wenig Augenmerk auf die Bedürfnisse der potenziellen Nutzer_innen des Systems gelegt worden. Performance- und Usability-Tests wurde zu wenig Platz und Zeit eingeräumt. Der Start gestaltete sich - um mit einem Euphemismus zu sprechen - holprig. Einiges war in den Jahren 2006 bis 2010 an Kriseninterventionen nötig, um die Wogen zu glätten. 2008 wurde die Leitung von RAD, die ursprünglich die Dienstleistungseinrichtung Forschungsservice und Internationale Beziehungen innehatte, im Zuge von Entscheidungen zur Kompetenzbündelung dem alleinigen Verantwortungsbereich der Universitätsbibliothek Wien übergeben.

\section{Die Ablöse von RAD - Suche nach Alternativen}

Um Verbesserungen an RAD herbeizuführen, tagte eine universitätsweite Arbeitsgruppe Usability RAD, die insbesondere die Verbesserung der Usability im Visier hatte. Es wurde eine Reihe von Vorschlägen erarbeitet, von den Entwicklern vieles davon auch umgesetzt, allerdings wurde offensichtlich, dass enorme Ressourcen notwendig sein würden, um diese Verbesserungen umzusetzen und mit den gleichzeitig wachsenden Anforderungen Schritt halten zu können.

Mit der Neubesetzung der Leitung des Zentralen Informatikdiensts im Jahr 2010 wurde nach einigen Besprechungsterminen eine aus heutiger Sicht richtige Entscheidung getroffen, nämlich die Weiterentwicklung von RAD vorerst zu stoppen und den Markt nach den mittlerweile auf den Plan getretenen Systemen kommerzieller Anbieter zu sondieren.

In den Zielvereinbarungen Anfang 2011 wurde die Universitätsbibliothek Wien beauftragt, Alternativen zu RAD zu evaluieren und der Autor beauftragt, eine Taskforce CRISneu ${ }^{5}$ ins Leben zu rufen, die sich auf die Suche nach geeigneten Alternativen zu RAD machen sollte. Zum damaligen Zeitpunkt war definitiv noch nicht absehbar, ob es tatsächlich geeignete Produkte geben könnte, die den Anforderungen der Universität Wien standhalten könnten und wenn ja,

5 Mitglieder der Taskforce CRISneu waren folgende Personen: Michael Greil (UB), Marion Polaschek (ZID), Peter Marksteiner (ZID), Wolfram Seidler (UB), Hans Gartler (UB), Lucas Zinner (DLE Forschungsservice und Nachwuchsförderung). 
ob sich ihre Anschaffung angesichts knapper Ressourcen finanzieren lassen würde.

Die Taskforce verfasste nach intensiven Vorarbeiten 2011 eine umfangreiche Anforderungsanalyse für das Forschungsinformationsmanagement an der Universität Wien und führte im Anschluss daran eine Marktsondierung mit einer Reihe von Anbietern durch. Es galt die Prämisse, Wissenschafter_innen und andere Nutzer_innen von Anfang an in den Prozess einzubinden, um höchstmögliche Transparenz gewährleisten zu können.

Im Jänner 2012 war die Taskforce eingeladen, ihre Ergebnisse in einer Sitzung dem Rektorat zu präsentieren. Nach der darauffolgenden Diskussion folgte die Universitätsleitung den Empfehlungen der Taskforce, RAD einzustampfen, sagte die Finanzierung zu und erteilte den Projektauftrag, ein neues zeitgemäßes Forschungsinformationssystem für die Universität Wien einzuführen.

Unmittelbar danach nahm das Projektteam mit der Unterstützung der Beschaffungsabteilung der Dienstleitungseinrichtung Raum- und Ressourcenmanagement 2012 das EU-weite Vergabeverfahren in Angriff. Nach der Veröffentlichung der Ausschreibung im Frühjahr 2012 folgte die Evaluierung der im Sommer eingegangenen Angebote im August. Kurz vor Bekanntgabe der $\mathrm{Zu}$ schlagsabsicht an die dänische Firma Atira A/S erreichte die Bewertungskommission die Nachricht, dass die charmante Firma in Aalborg mit ca. 30 Mitarbeiter_innen soeben an Elsevier verkauft worden war. Nach einer Garantieerklärung von Elsevier, sämtliche Leistungen zu den Vertragsbedingungen zu erbringen, wurde im September 2012 der Zuschlag erteilt.

\section{Implementierung von PURE als u:cris}

Ab Oktober 2012 implementierte das Projektteam in einem Kraftakt in nur knapp mehr als einem Jahr PURE an der Universität Wien unter dem Namen u:cris. RAD blieb in dieser Zeit noch in Betrieb und wurde im Mai 2013 tatsächlich vom Netz genommen. In den Sommermonaten wurde erst ein Beta-Test mit eingeladenen User_innen durchgeführt, anschließend ein Pilotbetrieb an fünf Fakultäten gestartet. Innerhalb von wenigen Monaten wurden fast 500 Personen durch Schulungsmaßnahmen gelotst.

Während des Projekts fanden zur Abstimmung mit dem Rektorat Sitzungen der Projektaufsicht statt, außerdem wurden mehrmals im Jahr Sitzungen des Sounding Boards mit Vertreter_innen der Fakultäten und Zentren abgehalten, um über den Projektfortschritt zu berichten. Gleichzeitig besuchte die Projektleitung in diesem Zeitraum alle Fakultäten und Zentren, um sie im persönlichen Gespräch auf den Umstieg auf $\mathrm{u}$ :cris vorzubereiten und eventuelle Fragen 
bzgl. Datenmigration zu klären und adäquate Erfassungs- und Validierungsprozesse zu etablieren.

Fast überrascht vom überaus positiven Useruptake waren wir überwältigt von der Resonanz, von der schieren Datenmenge, die sich in das System ergoss. Kurze Zeit später, im Frühjahr 2014, schafften wir zeitgerecht die erste Lieferung der Kennzahlen für die Wissensbilanz 2013 mit stark gesteigerten Zahlen. Während in dieser ersten Implementierungsphase der Fokus auf Publikationen und Vorträge auf Grund der gesetzlichen Berichtspflichten gelegt wurde, wurde in der darauf folgenden Ausbaustufe auch die Umsetzung einer universitätsweiten Dokumentation des Drittmittelprozesses in u:cris in Angriff genommen.

$\mathrm{u}$ :cris wurde in Folge zu einem integralen Bestandteil des universitätsweiten Berichtswesens für internes (Zielvereinbarungen, Evaluierungen) und externes Reporting (Wissensbilanz) entwickelt.

$\mathrm{u}$ :cris ist auch ein hervorragendes Bindeglied in der DLE-übergreifenden Kooperation. Am stärksten kommt dies bei der Zusammenarbeit mit der Dienstleistungseinrichtung Forschungsservice und Nachwuchsförderung zum Ausdruck, die für den Drittmittelprozess in $\mathrm{u}$ :cris fachlich verantwortlich zeichnet. $\mathrm{Zu}$ nennen ist hier auch die sehr gute Zusammenarbeit mit der Dienstleistungseinrichtung Finanzwesen und Controlling und mit der Besonderen Einrichtung für Qualitätssicherung.

\section{Über den Tellerrand hinaus: nationale und internationale Kooperationen}

Im Vergleich zum einsamen Dasein mit der Eigenentwicklung RAD, mit der wir völlig auf uns gestellt waren und wenig Gelegenheit hatten, über den eigenen Tellerrand hinauszublicken, hat sich mit dem Umstieg auf $\mathrm{u}$ :cris die Perspektive stark erweitert. Erstens pflegt das u:cris-Team natürlich Kontakt zur Entwicklerfirma in Aalborg (Dänemark) und zum Konzern in Amsterdam, zweitens existiert mit den entsprechenden Services an den Universitäten in Österreich und in Deutschland eine sehr enge Kooperation und reger Austausch, insbesondere mit jenen Universitäten und Forschungseinrichtungen, die auch PURE nutzen. Dazu zählen in Österreich die Technische Universität Graz, die Montanuniversität Leoben, die Universität Salzburg, in Deutschland die Deutsche Sporthochschule Köln, die Leibniz-Universität Hannover, die Universität Kiel, die Universität Hamburg, die Universität Lübeck, die Leibniz-Institute u.v.m. Im Bereich des Moduls Drittmittelverwaltung gibt es sogar eine globale Usergroup mit Universitäten in Australien, Kanada, Großbritannien, Russland und Belgien. Es hat sich als äußerst produktiv herausgestellt, Teil einer internationalen 
Community zu sein, um Problemlösungen zu diskutieren, Vorgangsweisen zu koordinieren oder etwa auch eine starke gemeinsame Verhandlungsposition gegenüber der Herstellerfirma zu entwickeln. Das geschieht neben der virtuellen Vernetzung bei Treffen der PURE Usergroup Deutschland-Österreich mit den Universitäten in Österreich und Deutschland oder bei der jährlichen PURE International Conference, bei der PURE-Anwender_innen aus der ganzen Welt zusammentreffen.

In der Anfangszeit der Forschungsdokumentation an der Universitätsbibliothek Wien schlug dem heutigen u:cris-Team Skepsis entgegen. Nicht nur einmal bekam man hier Sätze wie "Ihr seid's ja ned von der UB, ihr seid's von der Uni!« zu hören. Heute, fast 13 Jahre danach, reiht sich u:cris als fest verankertes Service in das Portfolio forschungsunterstützender Services der Universitätsbibliothek Wien ein und wurde über die Jahre sukzessive durch Aufbau und Entwicklung eines professionellen Teams ausgebaut. Aus einer One-Man-Show 2004 wurde eine Einheit an der Universitätsbibliothek Wien, die heute aus fünf Personen besteht.

$\mathrm{u}$ :cris läuft mittlerweile in einem robusten Regelbetrieb und besitzt über die ganze Universität Bekanntheit bei gleichzeitiger rascher, dynamischer Weiterentwicklung und einem stetigen Ausbau des Systems. Damit müssen auch die Service- und Supportangebote des u:cris-Teams Schritt halten. In diesem hochdynamischen Umfeld steigender Anforderungen ist es auch für das Team unerlässlich, sich ständig weiterzuentwickeln und zu qualifizieren.

Aus der heutigen Perspektive war es die richtige Entscheidung, die Agenden des Forschungsinformationsmanagements an der Universitätsbibliothek zu verankern, was vielleicht in den stürmischen Zeiten, als wegen teils tatsächlicher, teils vermeintlicher Unzulänglichkeiten des Vorgängersystems RAD viel Kritik einprasselte, vielleicht nicht immer ganz klar gewesen sein könnte. Dass Maria Seissl auch in schwierigeren Zeiten durch ihre Unterstützung Stabilität gewährleistete und zum richtigen Zeitpunkt bereit war, die Entscheidung zum Wandel mitzutragen, hat zum Erfolg des Projekts beigetragen. 


\section{NewsEye. Ein Horizon 2020-Projekt an der Universitätsbibliothek Wien}

Die beste Möglichkeit, mit zukünftigen Entwicklungen umzugehen, ist sie selbst zu gestalten. Das Digitale wandelt beständig Forschungslabore und Werkzeuge der Wissenschafterinnen und Wissenschafter. Gerade die Services einer Universitätsbibliothek müssen mit den Anforderungen angesichts eines auflebenden internationalen Konkurrenzkampfes der Universitätsstandorte Schritt halten. Dafür braucht es das ständige und beständige Gespräch mit Forscherinnen und Forschern aus den unterschiedlichen Disziplinen. Einerseits um Entwicklungen, Anforderungen und Bedürfnisse in den Blick zu nehmen und andererseits um deren Expertise in die Entwicklungsprozesse der Services einbringen zu können. Diese Neuausrichtung und die Netzwerke, die sich diesbezüglich in der Universitätsbibliothek entwickelt haben, bringen in dieser Hinsicht Gewinn. Das Gespräch und die Expertise erweitern sich noch, wenn wie in folgendem Beispiel eines Projekts des Horizon-2020-Frameworks der Europäischen Union aus den Gesprächen ein Antrag und eine erfolgreiche Einreichung wird. Konkret werden hier zusammen mit internationalen renommierten Expertinnen und Experten Wege erkundet, die bestehenden Informationssysteme historischer Zeitungen wie zum Beispiel ANNO für einen breiteren Kreis von Wissenschaftsdisziplinen zugänglich zu machen und bei der Erschließung und Beforschung behilflich zu sein.

Der Universitätsbibliothek Wien ist es gelungen, diesen strukturellen Wandel der Forschungslandschaften als Entwicklungsmöglichkeiten der Dienstleistungseinrichtung zu begreifen. Deswegen war und ist es ein Leichtes, das Forschungs- und Entwicklungsprojekt Newseye an die Universitätsbibliothek anzudocken und die Ergebnisse und internationalen Verbindungen in den laufenden Betrieb zu integrieren.

Im Folgenden werden die zentralen Punkte des 3-Jahresprojekts, das am 1. Mai 2018 gestartet wurde, dargestellt. Mit konkreten Ergebnissen für Forscherinnen und Forscher und Nutzerinnen und Nutzer ist ab Mitte der Projektzeit zu rechnen: 
Zeitungen sammeln Informationen über kulturelle, politische und gesellschaftliche Ereignisse. Seit ihren Anfängen im 17. Jahrhundert verzeichnen sie Milliarden von Begebenheiten, Geschichten und Namen in fast jeder Sprache, jedem Land und von jedem Tag. Zeitungen waren und sind zudem ein Mediensystem, das öffentlich und politisch Meinung macht. In den letzten Jahrzehnten wurden Millionen von Zeitungsseiten aus europäischen Bibliotheken digitalisiert und online verfügbar gemacht. Die Nachfrage nach historischen Zeitungen bleibt groß. Während die breite Öffentlichkeit allgemeines Interesse an dieser historischen und kulturellen Ressource zeigt, ist sie für das Bildungssystem wie auch für viele Geistes- und Kulturwissenschafterinnen und -wissenschafter von weitreichender Bedeutung.

Das Projekt Newseye ${ }^{1}$ hat sich zum Ziel gesetzt, den Zugriff auf und die Orientierung in diesen Zeitungen zu vereinfachen und entwickelt dafür einen automatischen Forschungsassistenten, der auch auf künstlicher Intelligenz basieren wird. Die Basis für diesen Forschungsassistenten wird in enger $\mathrm{Zu}-$ sammenarbeit von Informatikerinnen und Informatikern und digitalen Geisteswissenschafterinnen und -wissenschaftern gelegt. Grundlage dieser Arbeit bietet ein Testkorpus, das von 1850 bis 1950 reicht, Analysen der Artikeltrennung, automatischer Texterkennung und des Layouts durchläuft (Universitäten Innsbruck bzw. Rostock) und die digitalen Zeitungen der Nationalbibliotheken von Finnland, Frankreich und Österreich umfasst. Historiographische Forschungsfälle und Forschungsfragen bilden dann eine Matrix, auf der Methoden und Werkzeuge für eine effektive Durchsuchbarkeit und Nutzung mittels neuer Technologien und »Big Data«-Ansätze von den Informatikgruppen in La Rochelle und Helsinki entwickelt werden.

Die historischen Phänomene und Entwicklungen, die im Fokus der digitalen Geisteswissenschafterinnen und -wissenschafter in diesem Projekt stehen, sind Nationalismus und Revolutionen, Geschlechterfragen, Medien und Journalismus sowie Fragen der Migration. Die entwickelten Fragestellungen rund um diese Themenfelder werden so formuliert, dass sie mit herkömmlichen Suchwerkzeugen bisher nur sehr umständlich oder schlicht gar nicht zu stellen und schon gar nicht zu beantworten waren.

Den zukünftigen Nutzerinnen und Nutzern soll dann auch mit Hilfe von Ansätzen aus der Forschung zur Künstlichen Intelligenz ein digitaler Forschungsassistent zur Verfügung stehen, der Hinweise, Zusammenhänge und Kontexte für unterschiedliche Anforderungen liefern kann.

Durch das Projekt sind verbesserte Prozesse in Texterkennung, Textanalyse, Verarbeitung natürlicher Sprache, computergestützter Kreativität und Erzeugung natürlicher Sprache zu erwarten. Die Forschung profitiert von Optical

1 https://www.newseye.eu [Letzter Zugriff: 11.09.2019]. 
Character Recognition (OCR)/automatischer Texterkennung, Artikeltrennung und der Verfügbarkeit von nützlichen Tools und Möglichkeiten zum Suchen und Durchsuchen großer Mengen an Text bzw. auch Bildmaterial.

Der Start des Projekts im europäischen Kulturerbe-Jahr 2018 ist ob der Zielsetzung des Projekts sehr passend und zeigt kulturpolitisch wohl weiter in die Richtung eines Individuums, das sich mit Hilfe von Technologien im eigenen kulturellen Erbe orientieren kann.

\section{NewsEye in a nutshell}

Laufzeit: 1.5.2018-30.4.2021. Forschungs- und Entwicklungsprojekt. Teilnehmende Institutionen: Universität Innsbruck, La Rochelle (Koordination) und Rostock (Informatik); Universitäten Helsinki, Innsbruck, Montpellier und Wien (Digital Humanities); Nationalbibliotheken von Finnland, Österreich, Frankreich. Budget: ca. 3 Millionen Euro. Das Projekt wird im Rahmen des Förderprogramms Horizon 2020 der Europäischen Union im Rahmen der Finanzvereinbarung Nr. 770299 gefördert. 
Open-Access-Publikation im Sinne der CC-Lizenz BY-NC-ND 4.0

(c) 2019, Vandenhoeck \& Ruprecht GmbH \& Co. KG, Göttingen ISBN Print: 9783847110989 - ISBN E-Lib: 9783737010986 


\section{Die Universitätsbibliothek Wien als Partnerin europäischer Projekte. Von den Anfängen bis OpenAIRE Advance}

Für Universitäten ist es schon lange eine Selbstverständlichkeit, sich um Drittmittelfinanzierungen zu bemühen, um international wettbewerbsfähig zu bleiben. Die Einwerbung dieser Gelder wird sowohl über nationale Fördergeber wie den Fonds zur Förderung der wissenschaftlichen Forschung (FWF) als auch über die Europäische Kommission im internationalen Kontext durchgeführt. In der Regel wird in Form von Projekten, die mehrere Partnerinstitutionen vereinen, themenspezifische aktuelle Forschung betrieben, deren Finanzierung anders nur schwer möglich wäre. Dieser Trend macht auch vor den Dienstleistungseinrichtungen der Universität Wien nicht halt. Die Universitätsbibliothek Wien ist nun mittlerweile seit Mitte der 2000er Jahre bei der Einwerbung von Fördergeldern bei der Europäischen Union sowie bei nationalen Fördergebern erfolgreich. Dabei sind in erster Linie Infrastrukturthemen relevant, die keine klare Abgrenzung zur Wissenschaft bzw. dem wissenschaftlichen Arbeiten möglich machen. Viele Abteilungen der Bibliothek verstehen sich in erster Linie als Forschungsunterstützung und ihre Mitarbeiter_innen betreiben oft selbst wissenschaftliche Forschung im Rahmen ihrer Tätigkeiten. In diesem Text wird speziell die Beteiligung an EU-geförderten Projekten der Universitätsbibliothek Wien beleuchtet.

Als Gründungsmitglied des »E-Books on Demand (EOD)«-Netzwerkes im Jahr 2006 hat die Universitätsbibliothek Wien erste Fördergelder von Seiten der Europäischen Kommission erhalten. In der ersten Projektperiode, die bis 2008 andauerte, wurde im Rahmen des "eTEN-Programms « ${ }^{1}$ die Erstfinanzierung des Netzwerks ermöglicht. Später ist über das »Culture Programm ${ }^{2}$ ein Nachfolgeprojekt erfolgreich gewesen, welches nach einer Verlängerung im April 2014 auslief. Dies bedeutete aber keineswegs das Ende des EOD-Netzwerkes: inzwischen bieten 40 Institutionen aus zwölf europäischen Ländern dieses Service der

1 https://ec.europa.eu/digital-single-market/news/eten-programme [Letzter Zugriff: 28.01. 2019].

2 https://eacea.ec.europa.eu/sites/2007-2013/archiving [Letzter Zugriff: 28. 01.2019]. 
Digitalisierung von Büchern aus der Zeit von 1500 bis 1900 an. $^{3}$ An der Universitätsbibliothek Wien ist diese Serviceleistung nicht mehr wegzudenken und wird dementsprechend intensiv nachgefragt.

Von Jänner 2010 bis Jänner 2013 war die Universitätsbibliothek Wien Partnerinstitution im Projekt »New Library Services of Western Balkan Universities (NLSWBU) « ${ }^{4}$, welches über das »Tempus-Programm ${ }^{5}$ finanziert wurde. Die Universitätsbibliothek Wien steuerte dazu ihre Expertise im Bereich des Repositorium-Managements und Trainings von Bibliotheksmitarbeiter_innen bei.

Ein weiteres EU-finanziertes Projekt mit Beteiligung der Universitätsbibliothek Wien ist "Europeana Libraries « ${ }^{6}$, das sich über eine Laufzeit von Jänner 2011 bis Dezember 2012 erstreckte und über das ICT-PSP Programm ${ }^{7}$ abgewickelt wurde. In diesem Projekt wurden Sammlungen - wie etwa Fotos des Instituts für Kunstgeschichte - oder Materialien der Zentralbibliothek für Physik wie der Nachlass von Nobelpreisträger Erwin Schrödinger (1887-1961) oder von Hans Thirring (1888-1976) - digitalisiert, in das Repositorium PHAIDRA gestellt und mit Metadaten versehen. Im Anschluss wurden diese Objekte über eine Schnittstelle an Europeana ${ }^{8}$ geliefert. Damit stehen diese Objekte einer breiten Öffentlichkeit zur Verfügung und sind als europäisches Kulturgut digital langzeitarchiviert.

Im Juni 2015 startete das Projekt "LEARN (Leaders Activating Research Networks) « ${ }^{9}$ im Rahmen des Programms Horizon $2020^{10}$ mit einer Laufzeit von 24 Monaten. Das Ziel dieses Projekts war die Entwicklung eines Modells für eine Forschungsdaten-Policy für Forschungsinstitutionen. Es wurde ein begleitendes Toolkit erarbeitet und eine Trainingseinheit in fünf Sprachen erstellt, um ein möglichst breites, internationales Publikum zu erreichen.

Weiters ist die Universitätsbibliothek Wien seit dem ersten Projekt der »OpenAIRE-Initiative« (Open Access Infrastructure for Research in Europe) ${ }^{11}$, welche im Dezember 2009 im "FP7 Rahmenprogramm « ${ }^{12}$ startete, Partnerinstitution. Mittlerweile befindet sich das Konsortium in der vierten Projektperiode, die mit Dezember 2020 enden wird. Im Jahr 2015 wechselte die Förderlinie

3 https://books2ebooks.eu/de [Letzter Zugriff: 28.01.2019].

$4 \mathrm{http}: / /$ projects.tempus.ac.rs/en/project/663 [Letzter Zugriff: 30.01.2019].

5 https://eacea.ec.europa.eu/sites/2007-2013/tempus-programme_en [Letzter Zugriff: 28.01. 2019].

6 https://pro.europeana.eu/project/europeana-libraries [Letzter Zugriff: 30.01.2019].

7 http://ec.europa.eu/information_society/activities/ict_psp/about/index_en.htm [Letzter Zugriff: 30.01.2019].

8 https://www.europeana.eu/portal/de [Letzter Zugriff: 30.01 .2019$]$.

9 http://learn-rdm.eu/en/about/ [Letzter Zugriff: 30.01.2019].

$10 \mathrm{https}: / /$ ec.europa.eu/programmes/horizon2020/en [Letzter Zugriff: 31.01.2019].

$11 \mathrm{https} / / / w w w . o p e n a i r e . e u /[$ [etzter Zugriff: 01.02.2019].

12 https://ec.europa.eu/research/fp7/index_en.cfm [Letzter Zugriff: 31.01.2019]. 
von FP7 zu Horizon 2020. OpenAIRE ist ein paneuropäisches Forschungsinformationssystem mit Diensten zum Auffinden, Speichern, Verlinken und Analysieren von Forschungsresultaten über alle Disziplinen hinweg. Ziel ist die Förderung und Umsetzung der Open-Access-Richtlinien der Europäischen Kommission (EC) und des Europäischen Forschungsrates (ERC) im Bereich Wissenschafts- bzw. Forschungsförderung. OpenAIRE unterstützt das OpenAccess-Mandat und den Open-Research-Data-Piloten in Horizon 2020. 2010 ging das OpenAIRE-Portal online und ermöglichte erstmals, den entstandenen wissenschaftlichen Output von EU-geförderten Projekten im Gesamten darzustellen. Ein ebenso wichtiger Schritt war die Etablierung eines Allround-Repositoriums, welches für alle Wissenschafter_innen zur Verfügung steht. In $\mathrm{Zu}$ sammenarbeit mit der Europäischen Organisation für Kernforschung (CERN) entstand das europäische Repositorium ZENODO ${ }^{13}$.

Das Konsortium umfasst über 60 Partnerinstitutionen innerhalb der Europäischen Union und darüber hinaus. Damit gibt es in jedem teilnehmenden Land lokale Ansprechpersonen, National Open Access Desks (kurz NOADs), die OpenAIRE auf nationaler Ebene repräsentieren, Fragen beantworten, Informationen weitergeben und bei Bedarf gezielt an die zuständigen Stellen verweisen können. Darüber hinaus tragen die NOADs zur nationalen Vernetzung und Etablierung von Open Science bei. Die Nachhaltigkeit von OpenAIRE wird mit der Etablierung eines Rechtsträgers, derzeit in Form eines Non-Profit-Vereins, sichergestellt. Die Universität Wien hat im Juni 2019 als eine der ersten Partnerinstitutionen einen Mitgliedsvertrag unterzeichnet. Als wichtigste europäische e-Infrastruktur für Open Science wird OpenAIRE einen wertvollen Beitrag zur European Open Science Cloud (EOSC) ${ }^{14}$ liefern. Im November 2018 konnte die Universitätsbibliothek Wien in Zusammenarbeit mit dem Bundesministerium für Bildung, Wissenschaft und Forschung (BMBWF) den Launch des EOSC-Portals im Großen Lesesaal feiern. Dies wurde durch die österreichische EU-Ratspräsidentschaft im zweiten Halbjahr 2018 und die gute Zusammenarbeit der Universitätsbibliothek Wien mit dem BMBWF möglich.

Darüber hinaus ist die Universitätsbibliothek Wien aktuell an drei weiteren EU-geförderten Projekten beteiligt: Im Mai 2018 startete »NewsEye - A Digital Investigator for Historical Newspapers ${ }^{15}$ innerhalb des Programms Horizon 2020. Ziel ist die Weiterentwicklung bereits bestehender Konzepte, Methoden und Werkzeuge im Bereich der Digital Humanities, indem man einen erweiterten Zugang zu historischen Zeitungen für eine breite Öffentlichkeit ermöglicht. Mit neuentwickelten Tools und Methoden wird die Sicht auf historische

13 https://zenodo.org/ [Letzter Zugriff: 01.02.2019].

14 https://www.eosc-portal.eu/ [Letzter Zugriff: 01.02.2019].

15 https://www.newseye.eu/ [Letzter Zugriff: 01.02.2019]. 
Ereignisse und Entwicklungen verändert und dadurch eine neue Perspektive ermöglicht. Das Projekt "Social Sciences \& Humanities Open Cloud « (SSHOC) ${ }^{16}$ startete im Jänner 2019 ebenfalls im Rahmen des Programms Horizon 2020 und hat drei Jahre Laufzeit. Partner an der Universitätsbibliothek Wien ist "The Austrian Social Science Data Archive $\left(\right.$ AUSSDA) ${ }^{17}$. Ziel dieses Projekts ist die Gestaltung eines offenen Cloud-Ökosystems für den Bereich der Geistes- und Sozialwissenschaften. Ebenso startete die Universitätsbibliothek Wien als Projektpartnerin beim EOSC-Pillar ${ }^{18}$, ebenfalls im Programm Horizon 2020 angesiedelt, im Juli 2019. Hier werden nationale Open-Science-Bemühungen in Österreich, Belgien, Frankreich, Deutschland und Italien koordiniert und deren Beiträge zur Umsetzung der European Open Science Cloud sichergestellt.

Diese Beispiele von erfolgreichen Teilnahmen an EU-geförderten Projekten zeigen, dass auch in Zukunft internationale Partnerschaften angestrebt werden. Die Universitätsbibliothek Wien versteht sich selbst als eine innovative und zukunftsorientierte Einrichtung, die durch die Teilnahme an solchen Projekten nicht nur dringend notwendige weitere Ressourcen generieren, sondern - vielleicht noch wichtiger - internationale Kooperationen eingehen kann. Die Bandbreite der Themenbereiche reicht von Digitalisierung über Strategie- und Policy-Entwicklung bis hin zu Trainingsprogrammen im Bereich der Forschungsunterstützung, wobei hier Open Science eine wichtige Komponente darstellt. Das Einbringen der eigenen Expertise ist dabei ebenso wichtig, wie die Erfahrungen, die in einem internationalen Umfeld gesammelt werden können. Nicht zuletzt wird dadurch die übergeordnete Institution, nämlich die Universität Wien, durch diese Maßnahmen international als kompetenter Partner im Bereich Bibliothekswesen wahrgenommen.

16 https://www.cessda.eu/About/Projects [Letzter Zugriff: 01.02.2019].

$17 \mathrm{https} / /$ aussda.at/ [Letzter Zugriff: 01.02.2019].

18 https://www.eosc-pillar.eu/ [Letzter Zugriff: 28.08.2019]. 


\section{Gastbeiträge}

Open-Access-Publikation im Sinne der CC-Lizenz BY-NC-ND 4.0

(c) 2019, Vandenhoeck \& Ruprecht GmbH \& Co. KG, Göttingen 
Open-Access-Publikation im Sinne der CC-Lizenz BY-NC-ND 4.0

(c) 2019, Vandenhoeck \& Ruprecht GmbH \& Co. KG, Göttingen ISBN Print: 9783847110989 - ISBN E-Lib: 9783737010986 


\section{Wiener Lektionen. Rückblicke auf die Organisationsentwicklung der Universitätsbibliothek Wien}

\section{Arbeit als Lernerfahrung begreifen (und die eigene Persönlichkeit entwickeln)}

Anfang Mai 2005 bin ich als stellvertretender Leiter an die Universitätsbibliothek Wien gekommen. Damit sollte für mich sowohl eine spannende Arbeitsphase an einer der großen europäischen Bibliotheken als auch eine ausgeprägte Lernzeit an einer komplexen Organisation beginnen. Für meine späteren Aufgaben als Direktor der Universitätsbibliothek Mainz (2010-2018) und Direktor des Bibliothekssystems der Freien Universität Berlin (seit 2018) hatte ich wertvolle Kenntnisse und Erfahrungen sammeln dürfen. Zu danken habe ich hier vor allem meiner damaligen Chefin Maria Seissl, die mir als ihrem Stellvertreter großzügig Handlungsfreiräume eingeräumt hat und meine Arbeit mit ihrer reichen Bibliothekserfahrung stets unterstützend begleitet hat.

Wie lernt man Bibliothek? Lesen und Studieren helfen, aber hinreichend sind sie nicht. Lernen im Bibliothekswesen ist vor allem mit der Übernahme praktischer Aufgaben und der Bewältigung konkreter Herausforderungen verbunden. Bewusstes Lernen entsteht dann durch gezieltes Nachdenken über das eigene Tun, reflektierte Praxis sozusagen. Zudem erfolgt individuelles Lernen in Organisationen nicht isoliert, sondern sozial vermittelt und basiert insofern auf Kommunikation und Interaktion sowie Feedback und Rückkoppelung. Um unsere Bibliotheken zukunftsorientiert zu gestalten, muss unser Arbeiten gleichzeitig immer auch ein Lernen sein, das auf unsere Praxis erneuernd zurückwirkt. Mit Peter Senge gesprochen, gilt es, eine lernende Organisation auf den Weg zu bringen.

An der Universitätsbibliothek Wien haben wir 2008/09 ein großes Organisationsentwicklungsprojekt in Gang gebracht. Es sollte die strategische Ausrichtung unserer Bibliothek profilieren sowie neue Produkte und Services generieren. Eigentlich entscheidend waren aber nicht so sehr die Gegenstände unserer Aktivitäten, sondern bislang kaum erprobte Arbeitsweisen, die - jenseits 
hierarchischer Strukturen - Offenheit, Experiment und Selbstorganisation fokussierten. Diese Arbeit an der neuen Organisationskultur verlangte und förderte die Entwicklung der eigenen Persönlichkeit, um die Diversität der zahlreichen unterschiedlichen Stimmen in einen produktiven Dialog zu bringen. Besonders diese damit verbundenen Erfahrungen waren (und bleiben) für mich insofern wertvoll, als man als Führungskraft größerer Einrichtungen weniger auf fachliche Expertise, sondern vielmehr auf sozial-kommunikatives Vermögen aufbauen muss.

\section{Menschen zur Zusammenarbeit gewinnen (und Verantwortung teilen)}

Wissenschaftliche Bibliotheken haben sich im 19. Jahrhundert professionalisiert. Um ihre immensen und scheinbar endlos wachsenden Druckschriftenbestände verwalten zu können, haben sie eine ausgefeilte Apparatur entwickelt. Dabei wurde ein differenziertes Set von Normen, Standards und Regelwerken ausgearbeitet, das von einschlägig ausgebildetem Personal angewandt wurde. Umgesetzt wurde diese Aufgabenstellung in hierarchischen Organisationsformen, die sich am Bürokratiemodell orientierten. Seine klassische Beschreibung findet sich bei Max Weber.

Organisationen sind aber keine Maschinen, sondern Organismen. Das Wichtigste sind nicht die Strukturen, Prozesse oder Kennzahlen, sondern die Menschen. Sie sind entscheidend für Erfolg und gute Laune. Insofern haben sich Führungskräfte vor allem den Menschen zuzuwenden, also den Mitarbeiterinnen und Mitarbeitern, den Kolleginnen und den Kollegen sowie den jeweils eigenen Vorgesetzten. Und Zuwendung meint hier Kommunikation, Partizipation und Interaktion, also Gespräch, Beteiligung und gemeinsames Handeln.

Für den Organisationsentwicklungsprozess an der Universitätsbibliothek Wien haben wir versucht, die Menschen ins Zentrum zu rücken. So haben wir für die Besetzung der Steuerungsgruppe quer durch die Organisation und die Hierarchieebenen eingeladen und der Selbstorganisation größtmöglichen Raum gegeben. Die grundsätzliche Ausrichtung des Gesamtprojekts basierte auf Partizipation. Möglichst viele Mitarbeiterinnen und Mitarbeiter sollten beteiligt werden. Gelegenheit dazu bot sich vor allem in den Arbeitsgruppen und in den Großgruppenveranstaltungen. Als Vorbereitung für das Projekt haben wir Workshops für alle Führungskräfte der Bibliothek veranstaltet. Dabei sollten das Führungsverständnis und die Führungskultur thematisiert werden, um Beteiligung, Dialog und Verantwortung zu stärken. 


\section{Organisationsentwicklung zur Daueraufgabe machen (und gemeinsam betreiben)}

Bibliotheken haben sich im Übergang vom Spätmittelalter zur frühen Neuzeit als gesamtgesellschaftlich relevante Institutionen zur Sammlung, Bewahrung und Bereitstellung öffentlicher Information zu etablieren begonnen. Im Verlauf des Modernisierungsprozesses der nächsten Jahrhunderte sollte sich diese Stellung festigen und zum Höhepunkt der Druckkultur im 19./20. Jahrhundert einen monopolartigen Charakter annehmen. Der gesellschaftliche Erfolg des Organisationstyps Bibliothek schien ungefährdet. Forciert wurde diese Illusion noch durch die Einbindung in den öffentlichen Dienst, der dauerhafte Existenz und Fürsorge zu versprechen schien.

Die digitale Transformation hat die Erfolgsgeschichte der Bibliotheken zuerst relativiert und dann aufgehoben. Bibliotheken haben ihre Monopolposition längst verloren und müssen am Informationsmarkt um ihre Marktanteile schonungslos gesagt: um ihr Überleben - kämpfen. Die Wettbewerbskräfte Michael Porter hat dazu den methodischen Rahmen geliefert - der Lieferanten (Stichwort: Elsevier), der Konkurrenten (Stichwort: Google), der Ersatzprodukte (Stichwort: Schattenbibliotheken), der Kunden (Stichwort: alternative Informationsbeschaffung im World Wide Web) und der potentiellen Mitbewerber (Stichwort: disruptive Technologien) sind mittlerweile so angewachsen, dass Bibliotheken keine Treiber, sondern vielmehr Getriebene der gegenwärtigen Dynamik sind.

Das Organisationsentwicklungsprojekt der Universitätsbibliothek Wien hat diese Situation ernst genommen und frühzeitig damit begonnen, das eigene Selbstverständnis zu prüfen, eine Neuausrichtung zu riskieren und Veränderungsprozesse zu mobilisieren. Dabei sollten die zahlreichen Einzelaktivitäten der Hauptbibliothek und der Fachbereichsbibliotheken unter eine gemeinsame Strategie gestellt werden. Erst diese Zusammenführung machte es möglich, das in formaler Hinsicht funktional einschichtige Bibliothekssystem auch inhaltlich kohärent zu gestalten. Und die stark partizipatorische Ausrichtung sollte die Wege dafür ebenen, Verantwortung zu teilen und Organisationsentwicklung zur gemeinsamen Sache zu machen, um die Selbstorganisation zu stärken. Darüber hinaus sollte vermittelt werden, dass Organisationsentwicklung als permanente Aktivität zu betreiben ist, Organisation als Prozess des Organisierens zu verstehen ist. Darüber hat Peter Weick geschrieben. 


\section{Sich vom Perfektionismus verabschieden (und die Betaversionen feiern)}

Eine der traditionellen bibliothekarischen Kardinaltugenden ist Genauigkeit. Vor allem die bibliographische Erfassung gedruckter Monographien erfordert Präzision im Detail. Denn selbst der kleinste Nachweisfehler im Katalog kann dazu führen, dass das sorgfältig ausgewählte Buch in den schier endlosen Regalreihen für Generationen neugieriger Leserinnen und Leser unauffindbar bleibt. Der bibliothekarischen Organisationslogik entsprechend wurde dieser Perfektionismus verallgemeinert und zu einem wesentlich bestimmenden Kulturfaktor gemacht. Dass Exaktheit als bibliothekarische Schlüsselqualifikation ein entscheidendes Kriterium bei der Personalauswahl wurde, sorgte dafür, dass sich das System entsprechend reproduzierte.

Mittlerweile erweist sich diese Nullfehlertoleranz, die für einen immer kleiner werdenden Teilbereich bibliothekarischer Praxis berechtigt sein mag, als robuste Blockade organisatorischer Entwicklung. Jegliches Experimentieren oder Pilotieren, jegliches Lernen am Weg ohne exakten Plan ist damit unter den Generalverdacht schlimmsten Dilettantismus gestellt. Das Reden über das Unfertige, das Unabgeschlossene und das Offene wird zum Risiko, weil es dazu einlädt, Fehler und damit Unzulänglichkeiten zu identifizieren.

Um diesen Kältetod in makelloser Perfektion abzuwehren, haben wir an der Universitätsbibliothek Wien im Rahmen unseres Organisationsentwicklungsprojekts das Verfahren des Prototyping, angelehnt an Otto Scharmers Theorie U, eingesetzt. Mit der Einführung von Prototypen als unmittelbaren Maßnahmen haben wir die Voraussetzungen geschaffen, um neue Handlungsmöglichkeiten $\mathrm{zu}$ entwickeln, $\mathrm{zu}$ testen, eventuell wieder fallen $\mathrm{zu}$ lassen oder zu realisieren. Damit sollte nicht nur die Zukunft konkret in die Organisation geholt, sondern auch das Mindset der Perfektion aufgebrochen sowie Baustellen und Betaversionen zum Normalfall erklärt werden.

\section{Weiterziehen, wenn man ausgiebig gelernt hat (und Neues erfahren will)}

Ende Oktober 2010 habe ich die Universitätsbibliothek Wien verlassen und bin an die Universitätsbibliothek Mainz weitergezogen. Freilich ist die Universitätsbibliothek Wien eine derart komplexe und kompetente Bibliothek, dass es an und mit ihr tendenziell endlos zu lernen gibt; zumal dann, wenn die Leiterin ihrem Stellvertreter diese Freiheit zum Lernen einräumt. Trotzdem kann der Moment eintreten, die eigenen Lernabenteuer an einem anderen Ort fortsetzen 
zu wollen. Auch das, nämlich den richtigen Zeitpunkt für den Weggang zu treffen, habe ich an der Universitätsbibliothek Wien gelernt. Aber Lernen ist ein mentaler Vorgang - unabhängig von der physischen Präsenz. Und so ist mir die Universitätsbibliothek Wien im Rückblick und in der Erinnerung noch immer eine wichtige Folie für organisatorisches Ausprobieren und also Lernen. 
Open-Access-Publikation im Sinne der CC-Lizenz BY-NC-ND 4.0

(c) 2019, Vandenhoeck \& Ruprecht GmbH \& Co. KG, Göttingen ISBN Print: 9783847110989 - ISBN E-Lib: 9783737010986 


\section{Claudia Hausberger}

\section{Lessons learned}

Vor fast 20 Jahren haben Maria Seissl und ich einander in Innsbruck kennengelernt, und seither hatten wir immer wieder beruflich und auch privat miteinander zu tun. Beruflich sind wir uns in den unterschiedlichsten Rollen begegnet: Wir waren Kolleginnen aus verschiedenen Abteilungen und später aus verschiedenen Bibliotheken, sie war Vortragende während meiner Bibliotheksausbildung und einige Jahre lang meine Vorgesetzte. Im Lauf der Jahre habe ich vieles von ihr gelernt, "lessons learned", die hier zusammengefasst werden sollen.

Maria Seissl wurde mir in meinem ersten Jahr als Bibliothekarin in Innsbruck von einer Kollegin während eines Mittagessens formlos mit den Worten »Das ist die Mia« vorgestellt. Diese Formlosigkeit war für mich umso überraschender, als es damals an der Universität Innsbruck eine historisch gewachsene Hierarchie gab, die sich unter anderem am Kaffeetisch der Hauptbibliothek widerspiegelte: Zuerst nahmen die Akademiker_innen dort ihren Kaffee ein, danach durften die sogenannten B-Leute - Formalerschließer_innen, Erwerber_innen und meine Wenigkeit - Kaffeepause machen. Als Akademikerin und Leiterin der Fachbibliothek für Germanistik und Anglistik begegnete mir Maria Seissl jedoch im Gegensatz zu anderen Akademiker_innen auf Augenhöhe, und wir entdeckten sogar eine Gemeinsamkeit: Drei Jahre meiner Kindheit verbrachte ich im selben Ort, in dem sie aufgewachsen war. So etwas verbindet, und heute in Wien genießen wir es, miteinander so sprechen zu können, wie uns die Tiroler Schnäbel gewachsen sind - dann »hoamelet« es.

\section{Lesson learned: Unterrichten}

Kurz darauf wechselte Maria Seissl nach Wien, aber zu meiner Freude und der vieler Kolleg_innen kam sie wieder, um anlässlich der bibliothekarischen Grundausbildung 2000/2001 in Innsbruck Englisch zu unterrichten. Als Kind der 1970er Jahre hatte ich bis dahin nur Frontalunterricht und meist strenge und 
unnahbare Lehrpersonen gekannt. Die Bibliotheksausbildung hingegen war ganz anders, denn die meisten meiner Vortragenden, oft Arbeitskolleg_innen, bemühten sich, angenehme Lernbedingungen für uns zu schaffen. Maria Seissl schaffte es, aus diesem insgesamt förderlichen Klima durch ihre didaktischen Methoden noch hervorzustechen. Natürlich erarbeiteten wir uns vor allem das englische Fachvokabular, aber oft spielerisch und in Gruppen; und einmal zeigte sie uns den Kinofilm Notting Hill in englischer Originalfassung. Obwohl dieser Film mit dem Bibliothekswesen gar nichts zu tun hat, ist er allen Kursteilnehmer_innen noch heute lebhaft in Erinnerung und hat unsere Englischkenntnisse mehr gefördert, als es eine streng fachliche, aber trockene Unterrichtseinheit vermocht hätte. Für meine eigene spätere Unterrichtstätigkeit habe ich daraus gelernt, andere Elemente in meinen Unterricht aufzunehmen und die Lehrinhalte spielerisch aufzubereiten. Als ich Maria Seissl Jahre später um Rat fragte, wie ich Berufsschüler_innen etwas mehr motivieren könne, gab sie mir bezeichnenderweise den Tipp: »Lass sie ein Kreuzworträtsel lösen!« Das tat ich dann tatsächlich auch, und die Schüler_innen hatten sichtlich Spaß an dieser Art der Wissensüberprüfung.

\section{Lesson learned: Mitarbeiter_innen in die Personalauswahl einbeziehen}

Aufgrund ihrer Unterrichtstätigkeit an der Universitätsbibliothek Innsbruck war Maria Seissl häufig in Innsbruck, und deshalb trafen wir uns immer wieder sporadisch, auch nach Abschluss meiner Ausbildung, dann aber eher privat. Dies änderte sich, als ich mich Anfang 2006 kurz entschlossen am letzten Tag der Ausschreibung um eine Stelle an der Universitätsbibliothek Wien bewarb. Am folgenden Tag kam ein Anruf aus Wien, und Maria Seissl fragte mich, ob es mir mit meiner Bewerbung ernst sei. Mir war es sehr ernst damit, das darauf folgende Bewerbungsgespräch in Wien war es deutlich weniger. Neben Maria Seissl war mein künftiger direkter Vorgesetzter anwesend, dem ich bei dieser Gelegenheit ausführlich über die Regeln eines typisch tirolerischen Kartenspiels Auskunft gab. Es war das entspannteste und lustigste Vorstellungsgespräch meines Lebens. Maria Seissl amüsierte sich sichtlich über den Gesprächsverlauf, und ich bekam die Stelle (wohl nicht nur wegen meiner ausgezeichneten Kenntnisse im Kartenspiel Watten). Später darauf angesprochen meinte sie, dass ich zwar ihre Wunschkandidatin gewesen sei, dass es aber der direkte Vorgesetzte gewesen sei, der mich unter den Bewerber_innen für seine Fachbereichsbibliothek ausgesucht hatte. Es war und ist ihr wichtig, künftige Vorgesetzte oder Kolleg_innen in 
die Personalauswahl miteinzubeziehen, und diesen Grundsatz habe ich von ihr übernommen.

\section{Lesson learned: Machen lassen}

Im April 2006 trat ich also meinen Dienst im Juridicum an der Fachbereichsbibliothek Rechtswissenschaften in der Zeitschriftenabteilung an. $\mathrm{Zu}$ meiner Freude erwies sich die Beziehung zu meinem Vorgesetzten weiterhin als von Humor geprägt, und zusätzlich zeigten sowohl er als auch Maria Seissl sich sehr offen gegenüber meiner Neigung, mich in diverse Projekte einzubringen. So beteiligte ich mich unter anderem an der Erstellung eines »Welcome Kit« für neue Mitarbeiter_innen, an der »fliegenden Zeitschriftenbearbeitung « und der Organisation von Abendveranstaltungen. Das brachte Abwechslung in den Arbeitsalltag und war sehr motivierend, und im Zuge dieser Projekte lernte ich zudem viele kompetente und liebenswerte Kolleg_innen des »Haupthauses« kennen und schätzen.

\section{Lesson learned: Arbeits- und Dienstrechtliches}

Durch eine Karenzierung ergab sich später für mich die Möglichkeit, meine Stelle für ein Jahr zu teilen und in diesem Zeitraum vormittags im Personalreferat der Bibliotheksdirektion zu arbeiten. Obwohl ich bereits eine fixe Ganztagsstelle hatte, reizten und interessierten mich die Aufgaben und die Thematiken Personal-, Dienst- und Arbeitsrecht. Viel von dem damals erworbenen Wissen in diesen Bereichen kommt mir heute noch im Arbeitsalltag zugute.

\section{Lesson learned: Personalplanung, Finanzen und SAP}

Verblüffend war für mich zudem die Tatsache, dass Maria Seissl sämtliche der damals ca. 300 Stellen bzw. Vollzeitäquivalente auswendig im Kopf hatte. Sie wusste immer genau, wer welche Karenzstelle innehatte und wie die Stellen aufgeteilt waren. Das war allein schon aufgrund der Anzahl der Mitarbeiter_innen ein Kunststück, und erschwerend kam hinzu, dass viele dieser Stellen geteilt und nochmals zusammengesetzt waren. Ebenso konnte ich aufgrund der räumlichen Nähe zum Sekretariat beobachten, dass sie bis ins Detail über die Finanzen der Universitätsbibliothek Bescheid wusste und zusätzlich die passenden SAP-Konten für jede Ausgabe auswendig kannte. 


\section{Lesson learned: Strategieentwicklung}

Am meisten für meine derzeitige Tätigkeit gelernt habe ich während meiner Mitgliedschaft in der Steuerungsgruppe für Strategieentwicklung der Universitätsbibliothek Wien, der ich zwischen 2009 und 2010 angehörte. Der Strategieentwicklungsprozess wurde von Maria Seissl initiiert, dauerte insgesamt zwei Jahre und war extern begleitet. Während dieser Zeit wurden entscheidende Weichen für die Zukunft der Universitätsbibliothek gestellt, und ich hatte zuerst als Mitglied der Steuerungsgruppe und anschließend als Leiterin der Entwicklung des Prototyps "Mentoring" an der Universitätsbibliothek Wien die einmalige Chance, an diesem aufregenden Projekt mitzuarbeiten. Auch viele andere Kolleg_innen bestimmten die Strategie wesentlich mit und lieferten konstruktiven Input in die Entwicklung von Prototypen, neuen Projekten und Serviceleistungen. Der Strategieentwicklungsprozess war zwar zeitaufwendig und arbeitsintensiv, aber von ungeheurem Nutzen für die Universitätsbibliothek Wien und deren Innen- und Außenwirkung.

\section{Lesson learned: Lachen schadet nie}

Vor Jahren habe ich Maria Seissl einmal erklärt, dass ich gerne ihr Gehalt hätte, aber nicht ihren Job. Seit 2012 bin ich Leiterin der Universitätsbibliothek der »Vetmeduni Vienna«, und alle meine an der Universitätsbibliothek Wien »lessons learned« sind für die Bewältigung meiner Aufgaben von großem Nutzen. Zudem unterstützte Maria Seissl mich von Beginn an als Mentorin, und noch heute frage ich sie ab und zu um Rat und erhalte kompetente und kluge Antworten. Unsere privaten und beruflichen Treffen sind sehr von ihrer optimistischen Geisteshaltung, ihrer Herzlichkeit und ihrem Humor geprägt. Als Beleg für ihren Humor mag gelten, dass sie auf meine oben erwähnte Aussage bezüglich ihres Gehalts und Jobs mit schallendem Gelächter antwortete.

Im Laufe der Zeit durfte ich viele Seiten an Maria Seissl kennenlernen, beruflich wie privat. Ich schätze sie in beiden Bereichen sehr, und sie war und ist mir fachlich wie auch menschlich ein Vorbild. 


\section{Strategisch denken, Veränderung ermöglichen, Teamarbeit unterstützen und individuelle Entwicklung fördern - Gelebte Grundprinzipien in der Bibliotheksleitung bei Maria Seissl}

Als ich Maria Seissl im Herbst 2008 im Zuge meines Vorstellungsgesprächs an der Universitätsbibliothek Wien erstmals begegnete, war mir noch nicht bewusst, dass die Art, wie sich dieses Gespräch gestaltete, beispielhaft dafür war, wie sie ihre Aufgabe als Bibliotheksleiterin sieht und ausübt - nämlich als

\section{MIA - Die Teamworkerin}

Mir gegenüber bei diesem Vorstellungsgespräch saßen Maria Seissl als Leiterin der Dienstleistungseinrichtung Bibliotheks- und Archivwesen (um es ganz korrekt zu benennen), Andreas Brandtner als ihr Stellvertreter und Tanja Fabian als Leiterin der Fachbereichsbibliothek Publizistik- und Kommunikationswissenschaft. Und das Bemerkenswerte an diesem Vorstellungsgespräch war die Tatsache, dass nicht nur Maria Seissl als DLE-Leiterin mit mir einen Dialog führte, sondern dass es tatsächlich ein Gespräch - mit unterschiedlichen inhaltlichen Schwerpunkten - mit allen drei Beteiligten war. Da ich in meiner beruflichen Laufbahn häufiger Bewerbungssituationen erlebt hatte, bei denen die anwesenden Kolleg_innen v.a. als Staffage dienten, das Gespräch aber ausschließlich die Leitung führte, empfand ich dies als wohltuende Abwechslung und sinnbildlich für ein Betriebsklima, in dem man gern tätig sein will. Dieser erste Eindruck bewahrheitete sich auch im erlebten Alltag - Maria Seissl ist keine Führungskraft, die sich im Sinne eines überkommenen heroischen Führungsideals als alleinige Gestalterin der Bibliotheksentwicklung inszeniert, vielmehr gibt sie den Initiativen, Ideen und Vorschlägen ihrer Mitarbeiter_innen und Führungskräfte viel Raum, hat ein offenes Ohr für konstruktive Veränderungsund Verbesserungsvorschläge. Dabei war mir zu Beginn meiner Tätigkeit an der Universitätsbibliothek Wien noch nicht bewusst, welche aufregende Entwicklungsphase wir alle die Möglichkeit hatten aktiv mitzugestalten - ein Prozess, der eine weitere beispielgebende Seite zeigt, nämlich 


\section{MIA - Die Ermöglicherin}

Da mit Maria Seissl und Andreas Brandtner seit Mitte der 2000er Jahre zwei managementorientierte Persönlichkeiten das DLE-Leitungsduo bildeten, entschlossen sie sich sehr bald dazu, einen umfassenden Strategieentwicklungsprozess in Gang zu setzen. Nach ersten erfolgreich umgesetzten Maßnahmen in den Bereichen Organisations- und Personalentwicklung wurde 2009 ein auf mehrere Jahre und umfassend partizipatorisch angelegter Strategieentwicklungsprozess in Gang gesetzt. Dieser wurde u.a. durch das Einsetzen einer Steuerungsgruppe realisiert, die bezüglich Altersverteilung, Geschlecht und Berufserfahrung sowie Zusammensetzung aus unterschiedlichen Universitätsbibliotheks-Bereichen sehr heterogen war, um möglichst die gesamte Dienstleistungseinrichtung zu repräsentieren. Zudem wurden drei Großgruppentage veranstaltet, an denen sich alle interessierten Mitarbeiterinnen und Mitarbeiter beteiligen und ihre Ideen einbringen konnten. ${ }^{1}$ Als innovationsförderndes Element wurde eine sogenannte »Ideendatenbank" eingerichtet: als interessant/ relevant/innovativ empfundene Vorschläge wurden durch Projektteams vorab als "Prototypen" umgesetzt, bevor die daraus gewonnenen Erkenntnisse über eine Übernahme in den Regelbetrieb entschieden. Dieses rasche, ziemlich unbürokratische Ins-Tun-Kommen sorgte dafür, dass die Aufbruchsstimmung und Energie, die durch diesen Beteiligungsprozess vielerorts merkbar war, nicht in Frustration auf Grund zu langen Aufschiebens umschlug. Dass angesichts dieser heterogenen Menge an kreativen Vorschlägen trotzdem das große Ganze nie aus dem Blick verloren wurde, sondern vielmehr sichergestellt wurde, dass sowohl für den laufenden Betrieb als auch für die neu entwickelten Services und Angebote entsprechend qualifizierte Mitarbeiter_innen eingesetzt werden, dafür sorgte als Leiterin der DLE Bibliotheks- und Archivwesen

\section{MIA - Die Strategin und Förderin}

Nach nur einjähriger Tätigkeit an der Universitätsbibliothek Wien bekam ich die Möglichkeit, zuerst gemeinsam mit dem Leiter des Teams Integrierte Medienbearbeitung, Christian Beiler, als erfahrenem Kollegen ein Team zu koordinieren, das den Prototypen »Weg womit? - Koordinierter Bestandsabbau « entwickelte. Daraus entstand ein durchaus umfangreiches Umsetzungsprojekt, das die

1 Siehe dazu u.a.: Andreas Brandtner: Bibliotheken der Zukunft. Strategieentwicklung an wissenschaftlichen Bibliotheken am Beispiel der Universitätsbibliothek Wien. Salzburg, Master Thesis 2010; Maria Seissl, Wolfram Seidler: Strategieentwicklung und Innovation an der Universitätsbibliothek Wien. In: Andreas Degkwitz (Hg.): Personal- und Organisationsentwicklung in Bibliotheken. Berlin: De Gruyter 2013 (= BMPF 2), S. 197-202. 
Praxistauglichkeit unserer erstellten Vorschläge überprüfen sollte. Dass ich dieses Projekt leiten durfte, empfand ich als großen Vertrauensbeweis. Diese Förderung meiner beruflichen Entwicklung setzte sich fort, indem mir Maria Seissl einige Zeit später die Karenzvertretung als Leiterin der Fachbereichsbibliothek Theater-, Film- und Medienwissenschaft anbot - für eine bibliothekarische Fast-noch-Anfängerin mit noch nicht einmal drei Jahren einschlägiger Berufserfahrung durchaus keine Selbstverständlichkeit. Generell hat Maria Seissl einen unglaublich guten Überblick über die Potenziale ihrer Mitarbeiter_innen - sowohl der schon länger Tätigen als auch der neuen Kolleg_innen, die oftmals zuerst mit befristeten Verträgen in den Beruf einsteigen. Sie tauscht sich dazu regelmäßig mit ihren Führungskräften aus, hat eine ausgeklügelte Planung, die sicherstellt, dass sie möglichst alle Kolleg_innen, deren Potenzial ihr förderungswürdig erscheint, fest an der Universitätsbibliothek Wien verankern kann - und vergisst darüber hinaus nicht, dass auch bereits länger tätige Mitarbeiter_innen Interesse an beruflicher Weiterentwicklung und fachlicher Weiterbildung haben. Dass es trotzdem den Einen oder die Andere gibt, dessen berufliche Laufbahn - so wie bei mir auch - sich an einer anderen Bibliothek fortsetzt, unterstützt

\section{MIA - Die Netzwerkerin}

Sich der Wichtigkeit beruflicher und persönlicher Netzwerke bewusst seiend, vermochte es Maria Seissl sogar, dem Weggang ihres Stellvertreters Andreas Brandtner zumindest einen positiven Aspekt abzugewinnen - „Wir werden ab jetzt zumindest hervorragende Verbindungen zur Universitätsbibliothek Mainz haben « formulierte sie 2010 bei der Verabschiedung. Dass sie seit Jahrzehnten in unterschiedlichsten Funktionen auch in der Vereinigung Österreichischer Bibliothekarinnen und Bibliothekare (VÖB) aktiv ist, versteht sich eigentlich von selbst und sei hier nur am Rande erwähnt. Und auch, als ich einen dringenden Termin erbat, um sie darüber zu informieren, dass ich die Leitung der AK Bibliothek Wien für Sozialwissenschaften übernehmen würde, begann sie das Gespräch zwar mit den Worten »Will ich wirklich hören, was du mir jetzt sagst?«, um sich dann aber sehr ehrlich mit mir über diesen Erfolg zu freuen. Es war klar, dass unsere gute Beziehung sicher nicht mit meinem Weggang enden würde.

Und das hat sich auch bewahrheitet. Dass mich - abgesehen von diversen Aufeinandertreffen zu beruflichen Anlässen wie Bibliothekartagen, VÖB-Sitzungen oder Lehrabschlussprüfungen - heute mit Maria Seissl als erfahrener Leiterin der größten österreichischen Universitätsbibliothek ein regelmäßiger und reger fachlicher Austausch verbindet und ich weiß, dass ich mich auch mit kurzfristig aufkommenden Anliegen jederzeit an sie wenden kann, verzeichne 
ich als großes Plus in meiner Aufgabe als Leiterin der AK Bibliothek. Aber mindestens ebenso freut es mich ganz persönlich, dass aus der ehemaligen Chefin auch MIA - die Freundin geworden ist, mit der ich mich auch außerberuflich hervorragend austauschen kann, und deren positive Lebensbetrachtung nach dem Motto »Always look on the bright side of life« mich als Persönlichkeit und Führungskraft sicher ebenso nachhaltig beeinflusst. 


\section{Zugabe}

Open-Access-Publikation im Sinne der CC-Lizenz BY-NC-ND 4.0

(c) 2019, Vandenhoeck \& Ruprecht GmbH \& Co. KG, Göttingen 
Open-Access-Publikation im Sinne der CC-Lizenz BY-NC-ND 4.0

(c) 2019, Vandenhoeck \& Ruprecht GmbH \& Co. KG, Göttingen ISBN Print: 9783847110989 - ISBN E-Lib: 9783737010986 


\section{Der UB-Chor. Eine Plattform für inneren Ausgleich und innerbetriebliche Vernetzung}

Die Existenz des Chores der Universitätsbibliothek Wien geht direkt auf einen Wunsch von Maria Seissl zurück, den sie im Sommer 2013 nebenbei gegenüber einem unserer musizierenden Kollegen, Kurt Schaefer, geäußert hat: Sie fände es schön, so hat sie ihm gesagt, wenn bei einer Weihnachtsfeier auch einmal gesungen würde. Ganz in der Logik bibliothekarischer Fachreferate hat Kurt diesen Wunsch dann an mich, den Leiter der Fachbereichsbibliothek Musikwissenschaft, herangetragen, und wie es der Zufall wollte, hat das einen lange gehegten Traum in mir angesprochen, mich einmal (nach Jahren als Chorsänger) als Chorleiter zu versuchen. So habe ich dann im Herbst bibliotheksweit die Einladung ausgesandt, dass sich doch für vier Proben zusammenfinden möge, wer Lust hat, drei Chorstücke bei der Weihnachtsfeier zu singen - und so fand es dann auch statt.

Dass es bei dieser Einmaligkeit bleiben sollte, wollte jedoch vielen von den 22 Gründungssänger_innen (und auch mir) nicht so recht gefallen. Wir sind daher nach Weihnachten übereingekommen, dass wir unser Chorsingen fortsetzen wollen, und auch diese Idee wurde durch die Direktion unterstützt.

Als eine Aktivität, die ganzheitlich ist wie kaum eine andere, beeinflusst Singen Körper, Seele, Sinne und Geist gleichermaßen: Es kann Kreislauf, Atmung, Stoffwechsel und das Immunsystem fördern, Konzentration, Achtsamkeit, Merkfähigkeit und Flexibilität üben und seelisch involvieren durch die Möglichkeit, sich auszudrücken und von der Musik berührt zu werden. Voraussetzung dafür ist freilich, dass man gerne singt. Aber dann wird nicht zuletzt während des Singens Stress und Anspannung abgebaut und Sänger_innen, die zudem Führungsrollen haben, können diese als Teil der Gruppe für zwei Stunden vollkommen ablegen.

Aus diesen Gesichtspunkten heraus wurde der Chor als eine fortlaufende Corporate Social Responsibility (CSR)-Maßnahme organisatorisch verankert und gilt seitdem als stehendes Angebot an alle Mitarbeiter_innen der Dienstleistungseinrichtung Bibliotheks- und Archivwesen. Im Zeitraum von September bis Juni findet dienstags eine Chorprobe statt, die allen daran Interessierten 
offensteht. Die Einbettung der Probenzeit in die Tagesmitte - statt wie bei fast allen Amateurchören am Abend - ist für viele Teilnehmer_innen eine wichtige Motivation gewesen, mit dem Chorsingen zu beginnen, oder ermöglicht es wegen familiärer Verpflichtungen überhaupt erst, dabei zu sein.

Der Chor ist aber auch Plattform des Austauschs in einer so großen Organisation wie der Universitätsbibliothek Wien, die nicht nur in lokaler Hinsicht teils sehr kleinteilig organisiert ist. Viele der Chormitglieder haben sich bei dieser Gelegenheit erst als Kolleg_innen kennengelernt, und es ist ein Netz regelmäßiger persönlicher Kontakte quer durch die Abteilungen entstanden, das es davor nicht gab, aus betrieblicher Perspektive rein zufällig ist und ungeahnte Wege informeller Kommunikation fördert.

Voraussetzung dafür, dass Chorsingen funktioniert, ist eine laufende Weiterentwicklung statt unzusammenhängender einzelner Singtermine. Während der Chor sein Potential als CSR-Maßnahme aus dem Singen selber schöpft, ist es gruppendynamisch bedeutsam, dass mit Auftritten vor Publikum auch klare Zielsetzungen verfolgt und erreicht werden. Gleichzeitig sind Auftritte, besonders vor der Kolleg_innenschaft der Dienstleistungseinrichtung Bibliotheksund Archivwesen, Werbung für die Teilnahme, Ausweis der ernsthaften Bemühungen in der Probenarbeit und nicht zuletzt auch Bereicherung für den Bibliotheksalltag.

Daher haben wir noch 2014 begonnen, eine Auftrittstätigkeit zu etablieren, die mittlerweile auch aus der Universitätsbibliothek hinausstrahlt, wie Auftritte im Postgraduate Center der Universität Wien oder beim Festakt »Österreich liest« des Österreichischen Büchereiverbands zeigen. Zum ersten Quinquennium des Chors wollen wir unsere bisherige Tätigkeit in diesem Rahmen, der Festschrift für die wesentliche Ermöglicherin unseres Betriebschors, erstmals dokumentieren.

\section{3/14}

19. Dezember: Erster Auftritt eines Chors bei der Weihnachtsfeier der DLE Bibliotheks- und Archivwesen

28. Jänner: Erste Probe als Chor der Universitätsbibliothek Wien

\section{4/15}

2. Dezember: Gestaltung des CSR-Tags der DLE Bibliotheks- und Archivwesen 18. Dezember: Gestaltung der Weihnachtsfeier der DLE Bibliotheks- und Archivwesen

2. Juli: Sommerkonzert (Hörsaal 1 des Instituts für Musikwissenschaft der Universität Wien) 
2015/16

17. Dezember: Gestaltung der Weihnachtsfeier der DLE Bibliotheks- und Archivwesen

3. März: Gestaltung des CSR-Tags der DLE Bibliotheks- und Archivwesen

15. Juni: Sommerkonzert "Day turns to Night and to Day« (Festsaal des Billrothhauses der Wiener Gesellschaft der Ärzte)

2016/17

5. Oktober: Gestaltung des musikalischen Rahmenprogramms beim Festakt »Österreich liest. Treffpunkt Bibliothek« auf Einladung des Büchereiverbands Österreichs im Stift Klosterneuburg

5. Dezember: Gestaltung der Weihnachtsfeier des Postgraduate Centers der Universität Wien

20. Dezember: Gestaltung der Weihnachtsfeier der DLE Bibliotheks- und Archivwesen

30. April: Teilnahme am Chortreffen in Nikitsch

14. Mai: Gestaltung der Konventmesse im Stift Altenburg

2017/18

4. Dezember.: Gestaltung der Weihnachtsfeier des Postgraduate Centers der Universität Wien

14. Dezember.: Adventkonzert, St. Gertrud, Wien-Währing

21. Dezember: Gestaltung der Weihnachtsfeier der DLE Bibliotheks- und Archivwesen

17. März: Gestaltung der Abendmesse in der Deutschordenskirche (Wien)

23. Juni: Sommerkonzert, Aula am Campus der Universität Wien

2018/19

3. Dezember: Gestaltung der Weihnachtsfeier des Postgraduate Centers der Universität Wien

10. Dezember: Adventkonzert, St. Gertrud, Wien-Währing

20. Dezember: Gestaltung der Weihnachtsfeier der DLE Bibliotheks- und Archivwesen

24. Mai: Frühlingskonzert »So Runs Our Life« (Aula am Campus der Universität Wien)

23. Juni: Gestaltung der Konventmesse im Stift Altenburg 
Open-Access-Publikation im Sinne der CC-Lizenz BY-NC-ND 4.0

(c) 2019, Vandenhoeck \& Ruprecht GmbH \& Co. KG, Göttingen ISBN Print: 9783847110989 - ISBN E-Lib: 9783737010986 


\section{Impressionen aus den Jahren 2004-2019}


Open-Access-Publikation im Sinne der CC-Lizenz BY-NC-ND 4.0

(c) 2019, Vandenhoeck \& Ruprecht GmbH \& Co. KG, Göttingen ISBN Print: 9783847110989 - ISBN E-Lib: 9783737010986 


\section{Abbildungen}

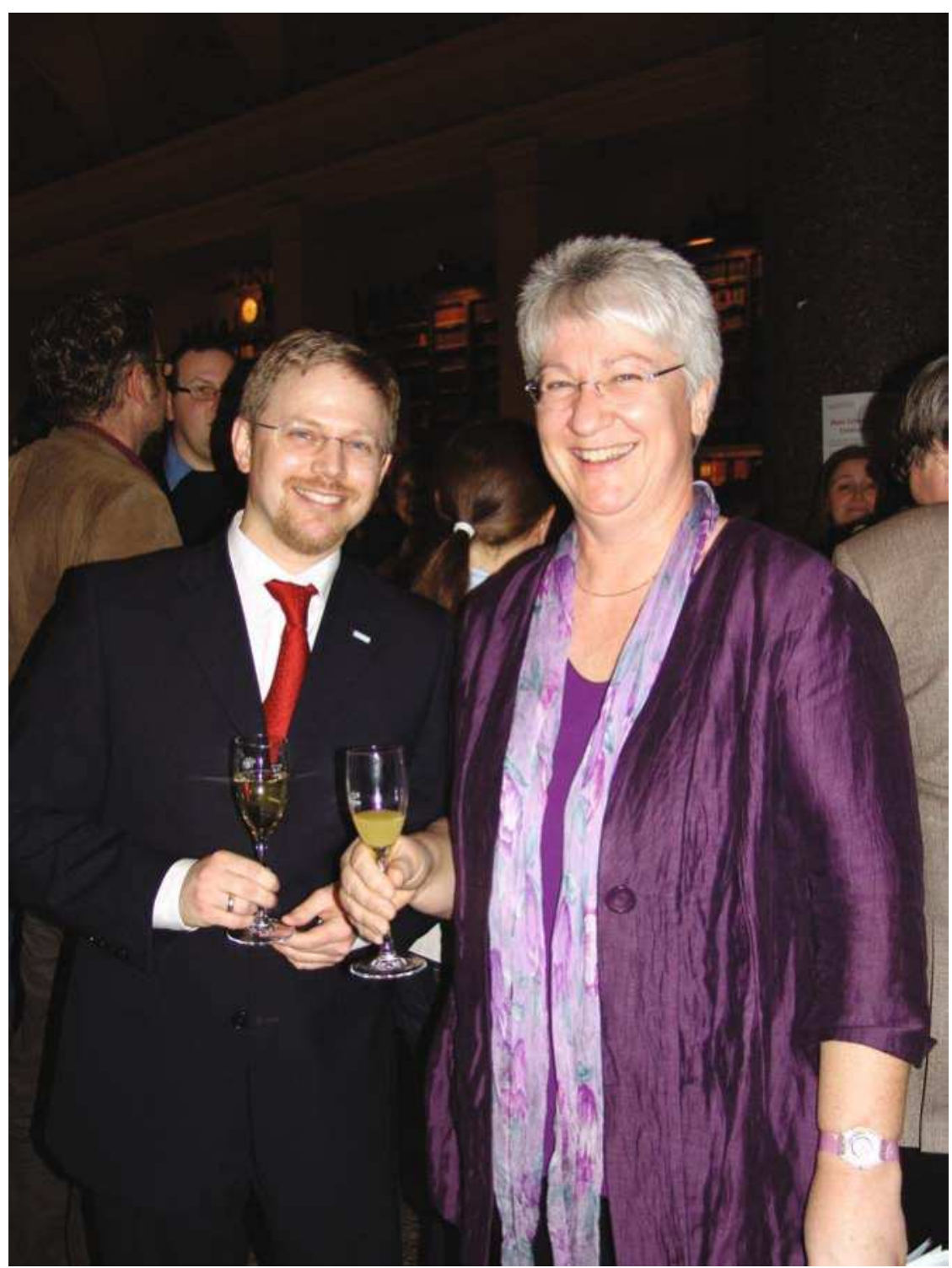

Abb. 1: Maria Seissl und Wolfgang Nikolaus Rappert bei der Weihnachtsfeier der DLE Bibliotheks- und Archivwesen 2010. Foto: Universitätsbibliothek Wien 


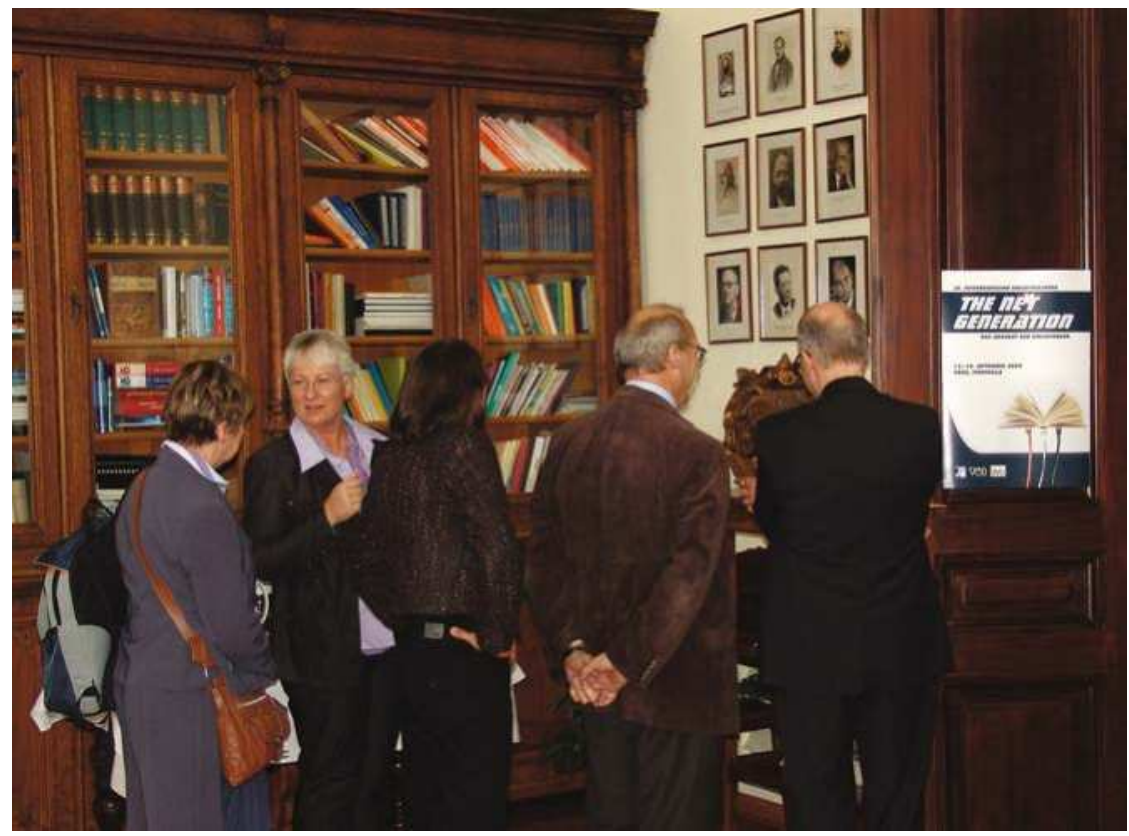

Abb. 2: Maria Seissl bei der Site Visit der Peers für die Evaluation 2009. Foto: Universitätsbibliothek Wien

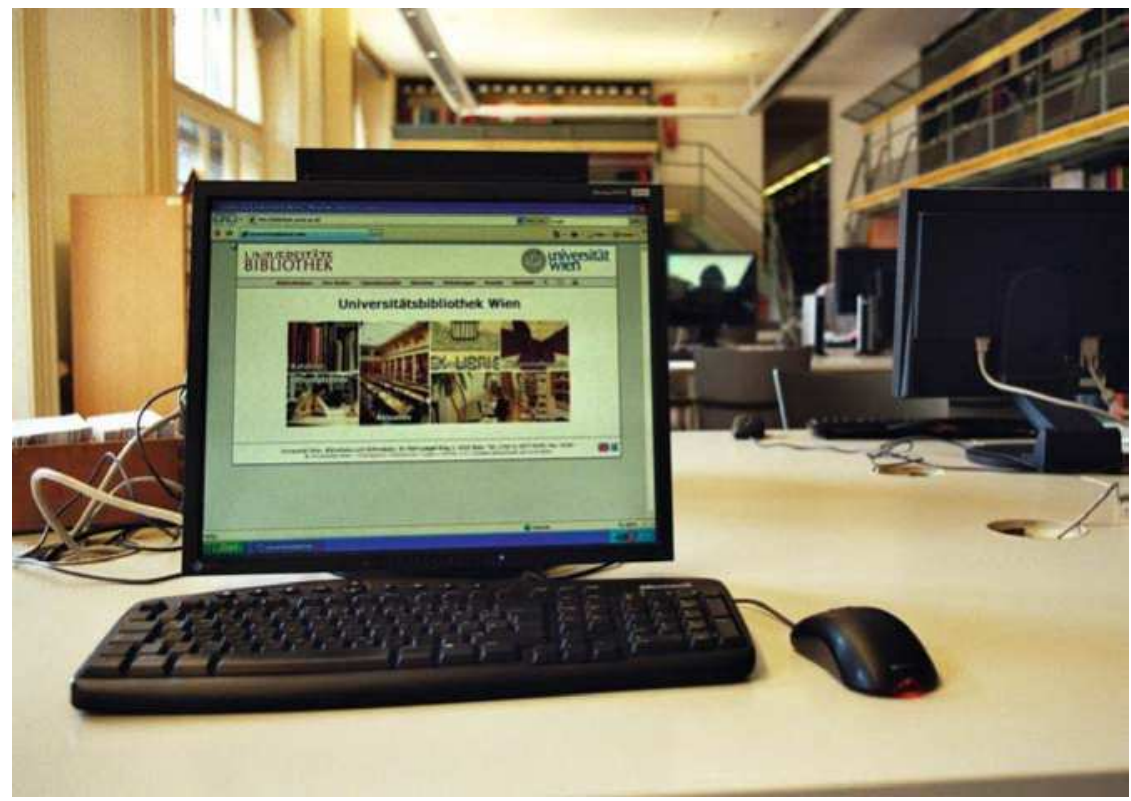

Abb. 3: Katalogsaal 1 der Hauptbibliothek 2009. Foto: Pflügl 


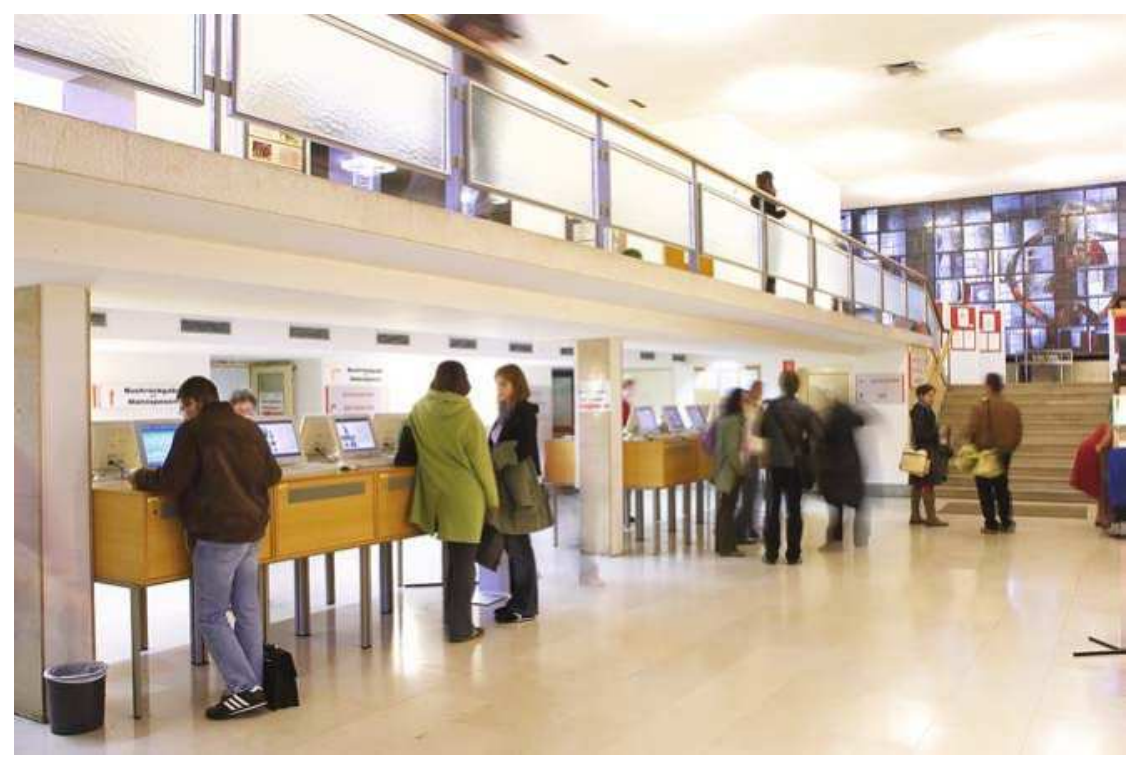

Abb. 4: Foyer der Hauptbibliothek 2006. Foto: Pflügl

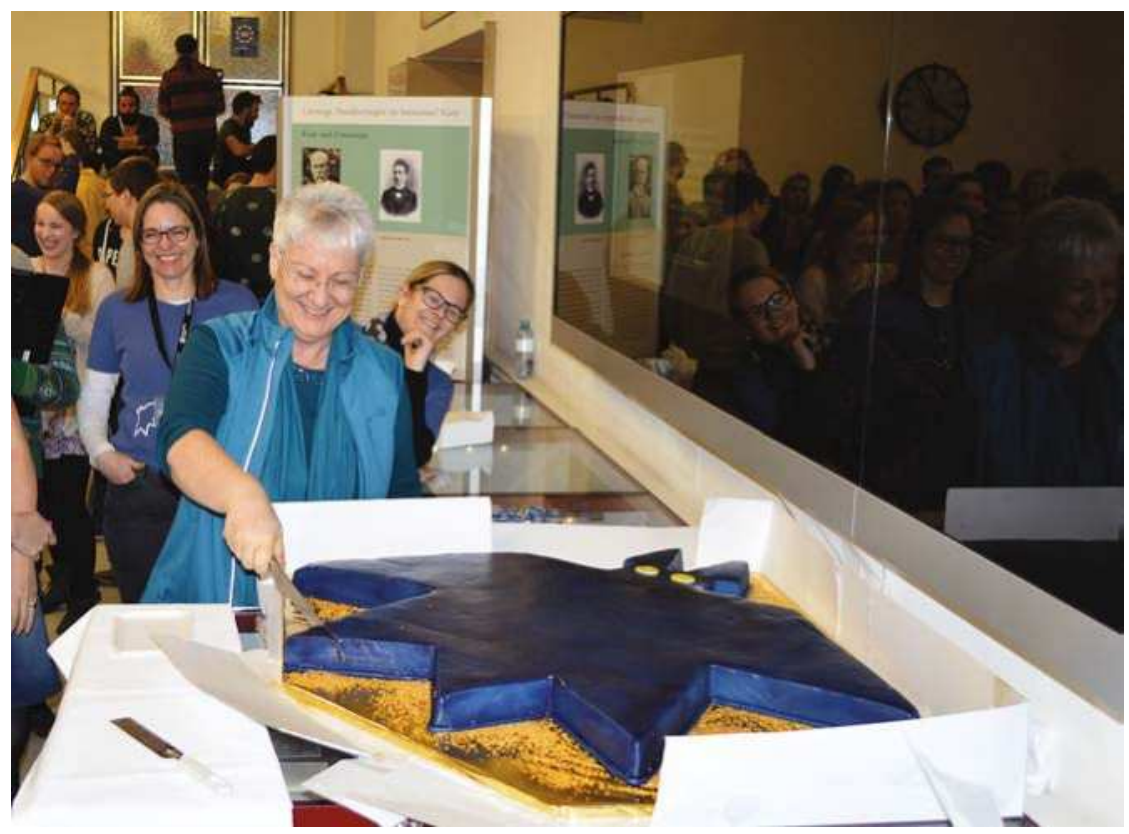

Abb. 5: 10. Nachtschicht@UB 2016. Foto: Universitätsbibliothek Wien 


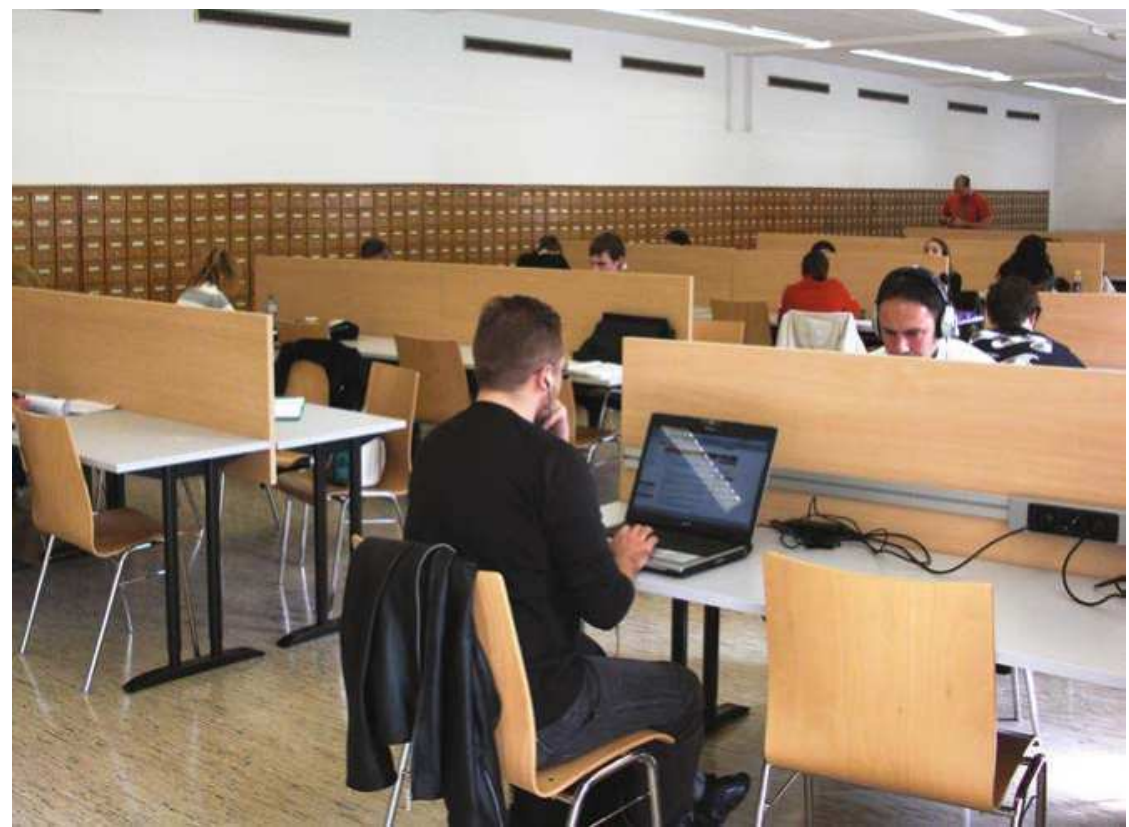

Abb. 6: Kleiner Lesesaal der Hauptbibliothek 2008. Foto: Universitätsbibliothek Wien

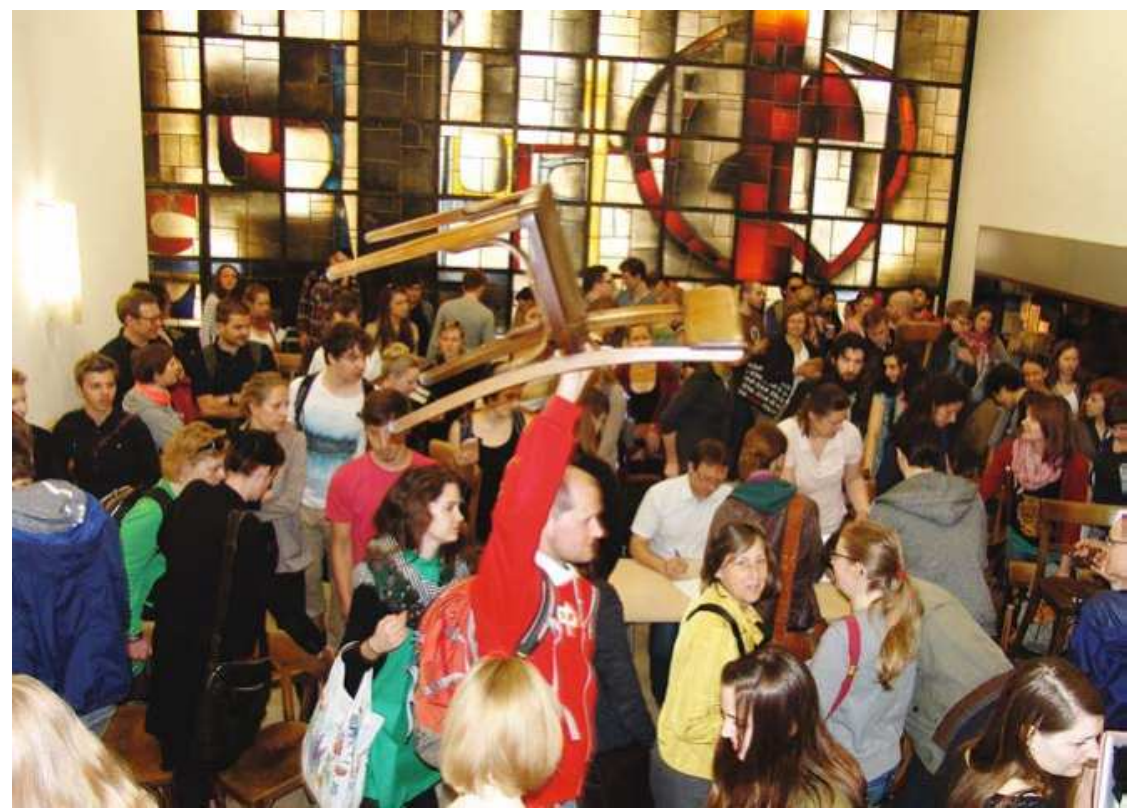

Abb. 7: Sesselflohmarkt an der Hauptbibliothek 2013. Foto: Universitätsbibliothek Wien 


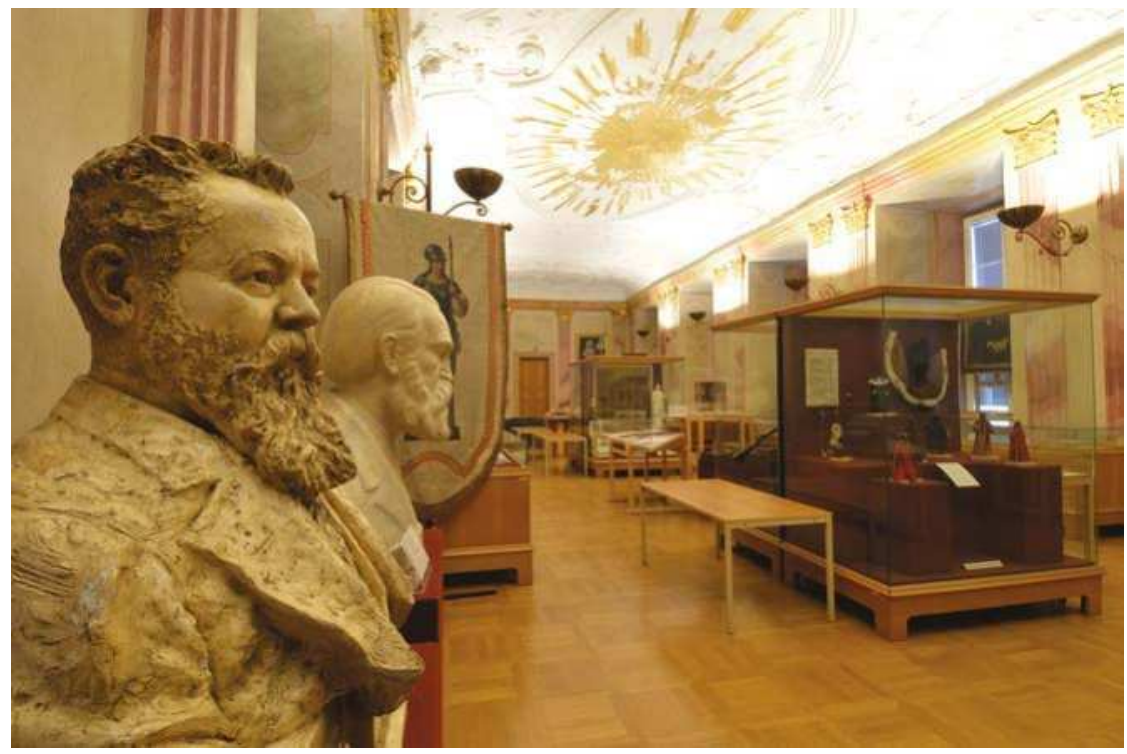

Abb. 8: Archiv der Universität Wien 2013. Foto: Universitätsbibliothek Wien

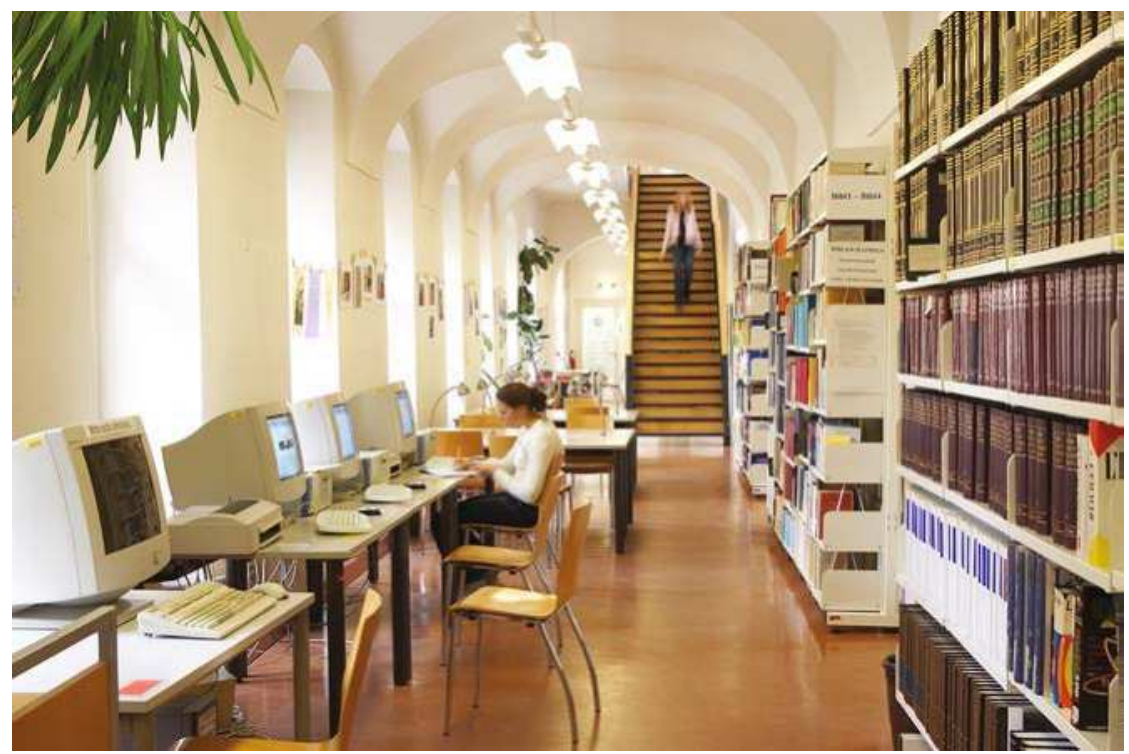

Abb. 9: Fachbereichsbibliothek Romanistik 2006. Foto: Pflügl 


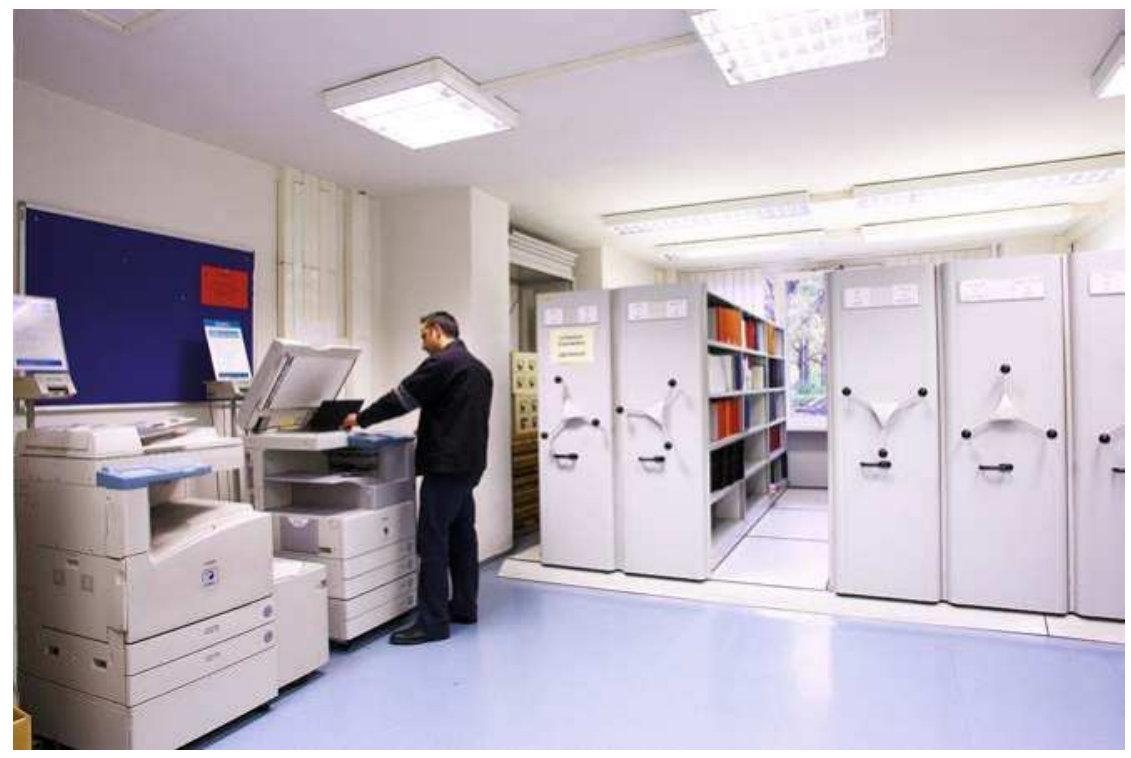

Abb. 10: Fachbereichsbibliothek Soziologie und Politikwissenschaft 2006. Foto: Pflügl

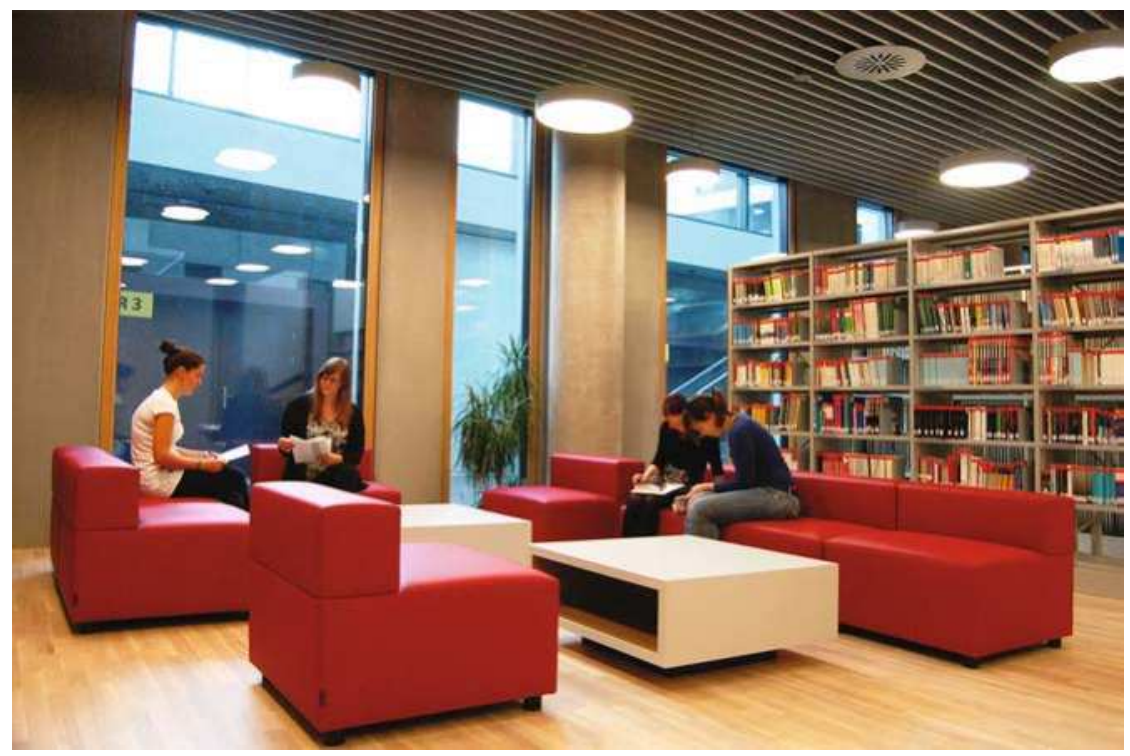

Abb. 11: Fachbereichsbibliothek Publizistik- und Kommunikationswissenschaft und Informatik 2012. Foto: Universitätsbibliothek Wien 


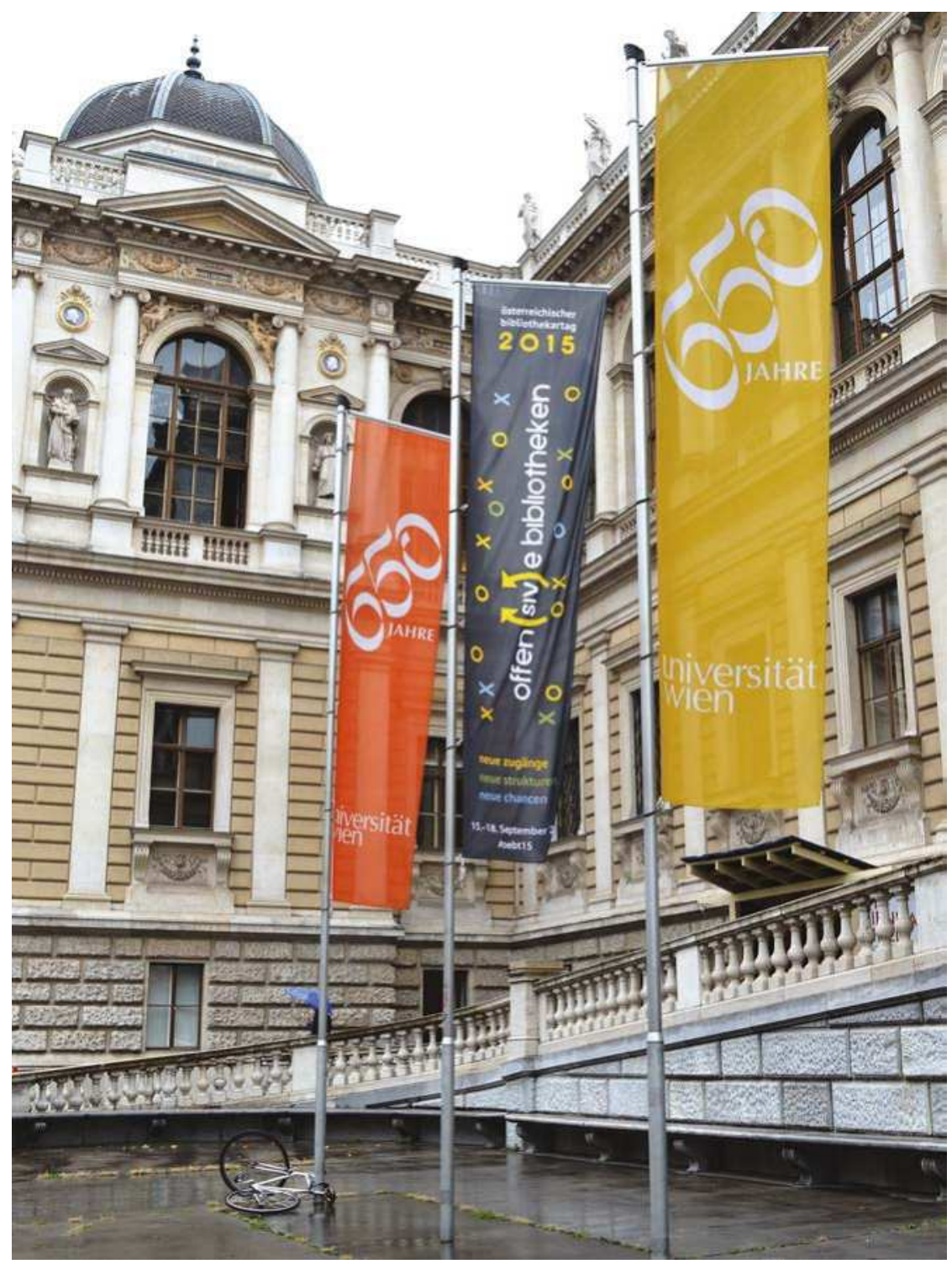

Abb. 12: Österreichischer Bibliothekartag 2015. Foto: Universitätsbibliothek Wien 


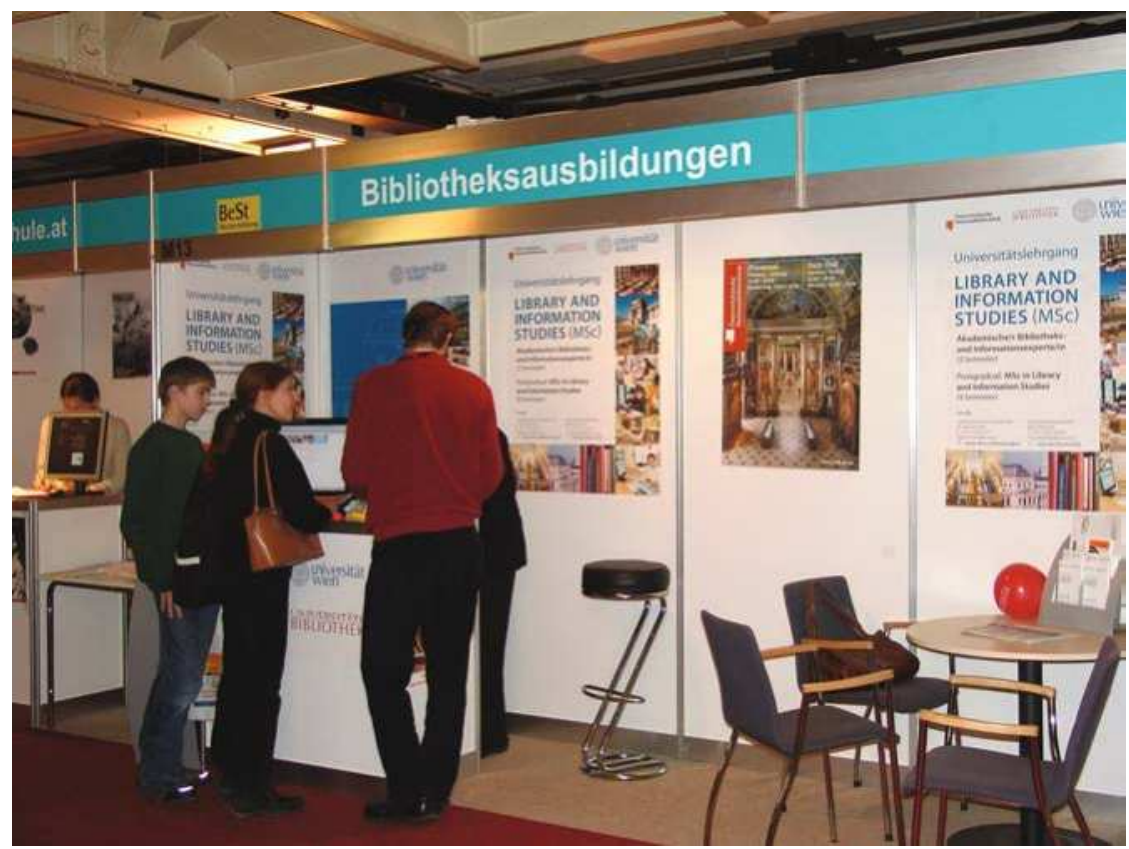

Abb. 13: BeSt (Messe für Beruf, Studium und Weiterbildung) 2009. Foto: Universitätsbibliothek Wien

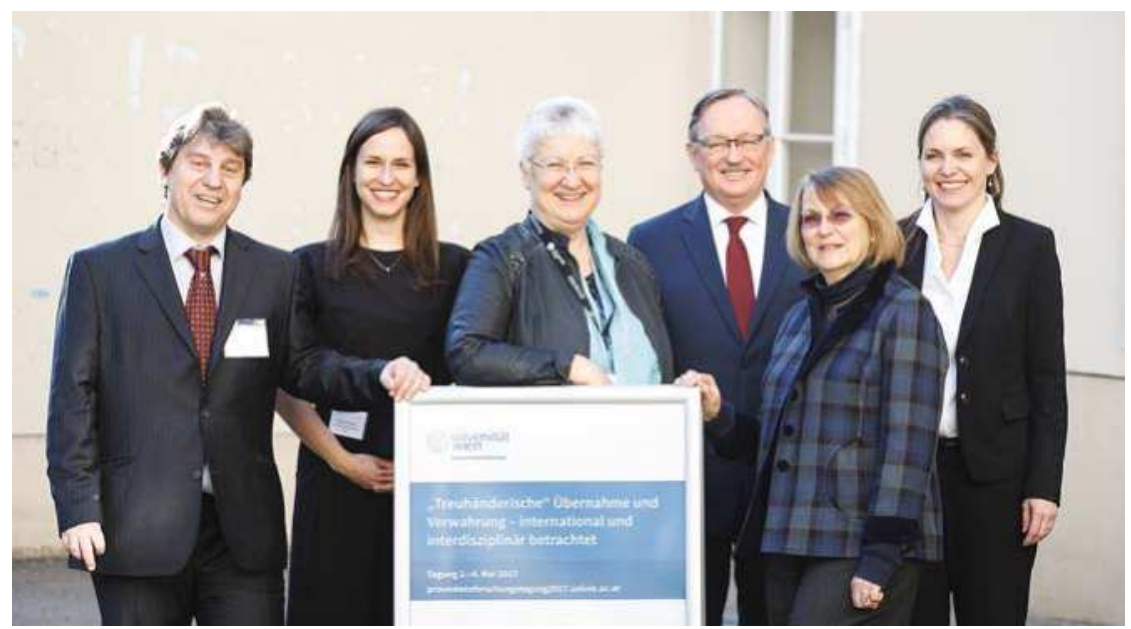

Abb. 14: Markus Stumpf, Olivia Kaiser, Maria Seissl, James D. Bindenagl, Vizerektorin Regina Hitzenberger und Christina Köstner-Pemsel bei der Eröffnung der Tagung »Treuhänderische Übernahme und Verwahrung« 2017. Foto: Josef Krpelan 


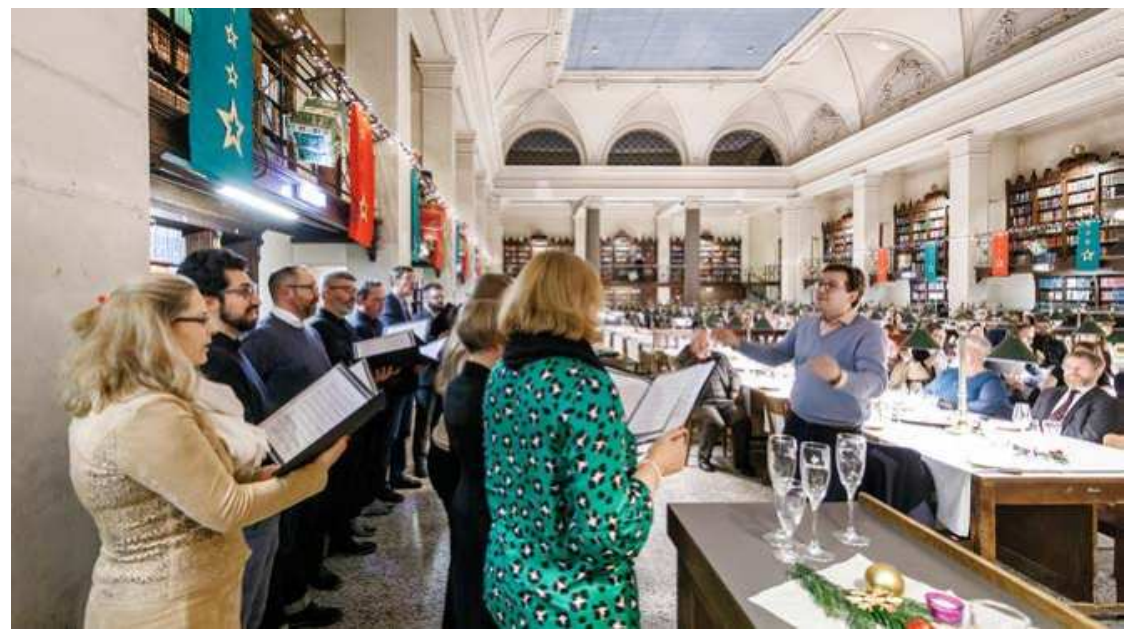

Abb. 15: UB-Chor bei der Weihnachtsfeier der DLE Bibliotheks- und Archivwesen 2018. Foto: derknopfdrücker.com

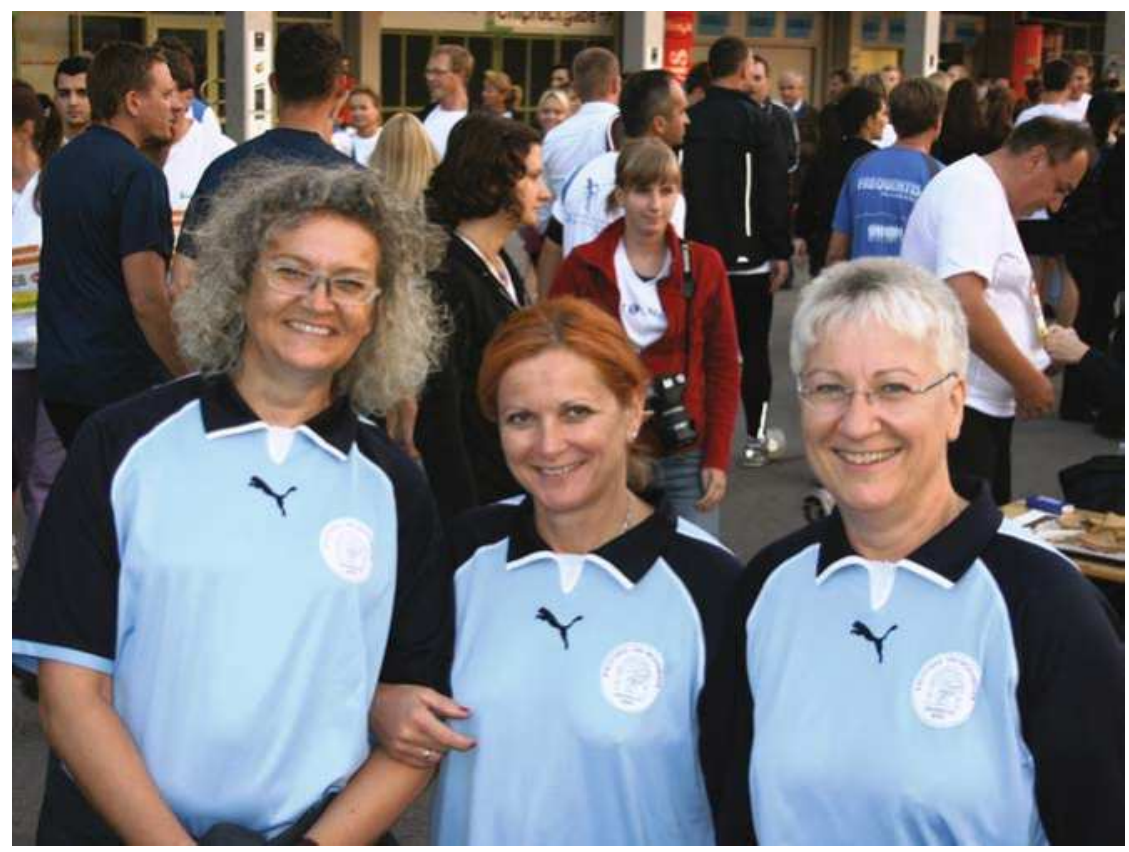

Abb. 16: Maria Seissl mit Claudia Hausberger und Christa Fried beim Business Run 2011. Foto: privat 


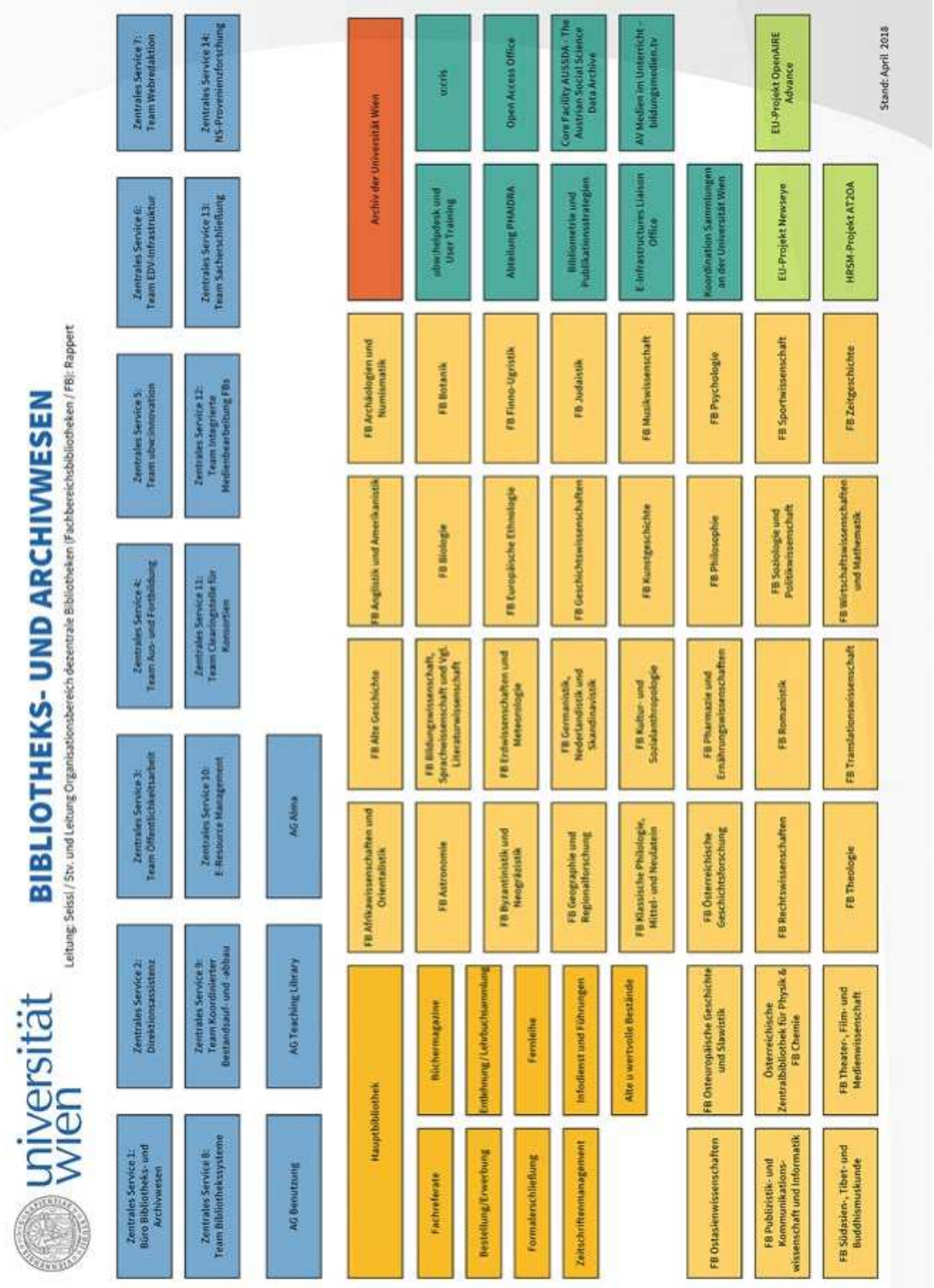

Abb. 17: Organigramm der DLE Bibliotheks- und Archivwesen, Stand: April 2018 


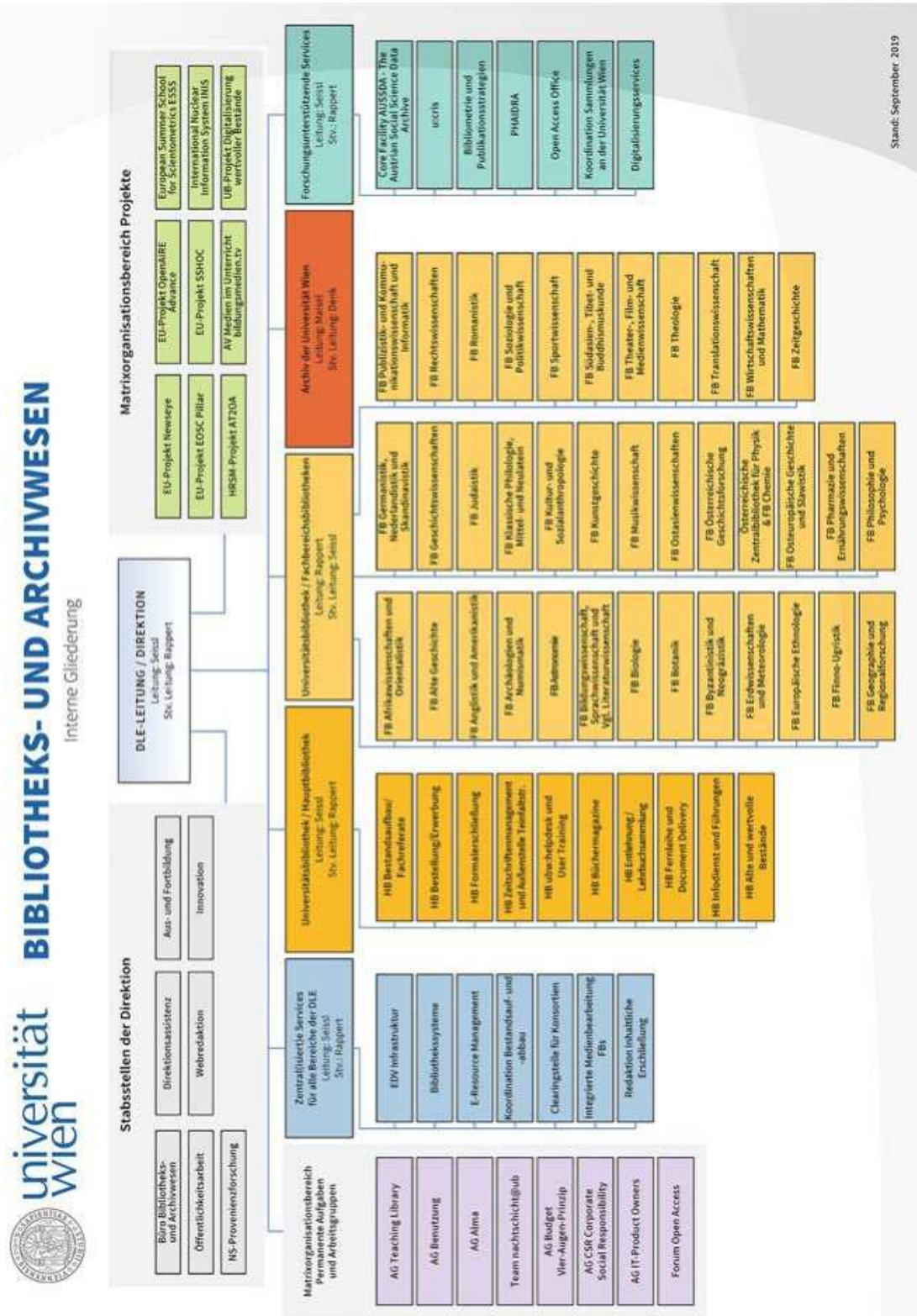

Abb. 18: Organigramm der DLE Bibliotheks- und Archivwesen, Stand: September 2019. Die Neugestaltung des Organigramms trägt mit einer differenzierten Darstellungsweise der Vielzahl an unterschiedlichen Aufgabenbereichen Rechnung. Es befindet sich zum Zeitpunkt der Drucklegung dieser Festschrift noch in einem Entwurfsstadium, veranschaulicht als Momentaufnahme aber gut, wie Maria Seissl die Organisation insgesamt entwickelt. 


\section{Maria Seissl: Organisatorin, Wissensmanagerin und Abenteurerin}

Simone Kremsberger am 14. Dezember 2004

Beim Versuch, den Kilimandscharo zu besteigen, hat sie auf $\mathbf{5 0 0 0}$ Meter umgedreht: "Die Höhe machte mir zu schaffen." Ansonsten ist sie keine, die kehrtmacht. Im Jahr 2000 startete Mag. Maria Seissl ihre Karriere an der Universitätsbibliothek Wien. Und die ging steil bergauf - seit November 2004 ist die Tirolerin Leiterin der DLE Bibliotheks- und Archivwesen.

Nach einem Jahr provisorischer Leitung steht Maria Seissl offiziell der DLE Bibliotheks- und Archivwesen der Universität Wien vor und ist verantwortlich für 56 bibliothekarische Standorte, 270 MitarbeiterInnen und natürlich die Bücher, ganze sechs Millionen Bände. Angesichts dieser beeindruckenden Zahlen bleibt Maria Seissl gelassen: "Ich lasse mich nicht leicht aus der Ruhe bringen." Die Herausforderung ihrer neuen Aufgabe macht ihr Spaß. "Man muss einen guten Überblick über das Gesamtgefüge der Universität und der DLE haben - dabei hat mir geholfen, dass ich ab 2001 als Stellvertreterin der früheren Bibliotheksdirektorin, Dr. Ilse Dosoudil, für den Dezentralen Bereich verantwortlich war." Die 45-jährige Tirolerin charakterisiert sich als teamfähig, geschickt in Verhandlungen und im Erkennen und Lösen von Problemen, die natürlich auch vor der UB nicht Halt machen.

\section{Die Organisatorin:}

\section{Entlehnabteilung und digitale Bibliothek als nächste Projekte}

"Ein großes Problem ist das enge räumliche Korsett", so Seissl. "Bibliotheken wachsen, und um gute Serviceleistungen zu erbringen, braucht man einfach Platz." In einem ersten Schritt will man sich 2005 der Raumsituation im Entlehnbereich der Hauptbibliothek widmen. Die Abteilung wird umgebaut, die Benutzerlnnen sollen bestellte Bücher selbst aus den Regalen nehmen und an Entlehnstationen verbuchen können. Ein weiteres Vorhaben ist die Weiterentwicklung der digitalen Bibliothek: Auch die Bestände, die bisher nur in Kartenkatalogen oder eingescannten Katalogen verzeichnet sind, sollen erschlossen werden. Bis Ende 2006 sollen alle Bestände an der Hauptbibliothek von 1932 bis 1989 erfasst werden. In einem zweiten Schritt will man das digitale Angebot über eine Portalsoftware zugänglich machen, mit einer Suchanfrage sollen alle Datenbanken durchsucht werden können.

Der Servicegedanke ihres Berufes ist Maria SeissI wichtig: "Bibliothekare sind Hüter eines großen Schatzes, nämlich von Wissen. Wir bringen Ordnung in die Informationsflut und vermitteln Informationskompetenz." Und das auch über die Universitätsgrenzen hinaus: "Wir wählen Literatur und Medien, die die Lehrenden, Forschenden und Studierenden der Universität brauchen, aber wir sind auch für die wissenschaftlich interessierte Öffentlichkeit da."

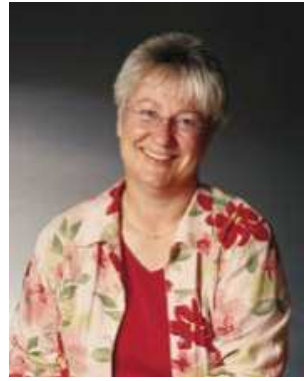

Foto: Niklas Jelinek 


\section{Die Wissensmanagerin: "Aus- und Weiterbildung ist mir wichtig!"}

Um effiziente Hilfestellung bei der Informationssuche geben zu können und rund 1,3 Millionen Entlehnfälle im Jahr zu bewältigen, sind gut geschulte Mitarbeiterlnnen vonnöten. Auf deren Aus- und Weiterbildung legt die UB-Chefin großen Wert: "Mit 'Forum Infodienst' haben wir ein gut koordiniertes internes Fortbildungsprogramm, innerhalb dessen MitarbeiterInnen ihre KollegInnen schulen und über Neuerungen informieren. Im Wintersemester 2004/05 haben wir den Universitätslehrgang 'Master of Science Library and Information Studies' eingeführt, um die Bibliotheksausbildung auf ein international anerkanntes Niveau zu stellen." Seissl, organisatorische Leiterin des Lehrgangs, wird selbst im Sommersemester 2005 Fachenglisch unterrichten. Damit erfüllt sich die studierte Anglistin zumindest teilweise ihren ursprünglichen Berufswunsch: Lehrerin.

Als Fremdsprachenassistentin in London, Lektorin für Deutsch als Fremdsprache an der University of Leeds und Lehrbeauftragte am Institut für Anglistik der Universität Innsbruck hat sie Unterrichtspraxis gesammelt. Der Wechsel ins Bibliothekswesen kam per Zufall: "Einen Monat nach meinem Studienabschluss wurde eine Fachreferentin für Anglistik an der UB Innsbruck gesucht. Ich habe mich beworben, die Stelle bekommen und bin im Bibliothekswesen gelandet." Aus dem Zufall entwickelte sich Freude am Beruf und eine lineare Karriere.

\section{Die Abenteurerin: Krimis und wilde Tiere}

Wenn Maria Seissl nicht arbeitet, liest sie gern ("Obwohl ich das als Bibliothekarin kaum zugeben kann, denn Lesen reicht nicht, um gut im Beruf zu sein."), und zwar Krimis zeitgenössischer englischer Autorinnen. Zu ihrer Krimileidenschaft hat sie eine eigene Theorie entwickelt: "Ich kenne sehr viele Kolleglnnen, die auch gerne Krimis lesen, und glaube, dass das mit dem Beruf zusammenhängt. In Krimis geht es darum, aus verwirrenden Informationen einen Fall zu lösen - und auch Bibliothekare versuchen stets, eine Lösung, eine Information zu finden." In der Literatur sind Bibliotheken ein beliebtes Motiv: "Es gibt viele literarische Darstellungen des Bibliothekarsberufs, oft diese traditionelle Darstellung der Bibliothekarin als strenger Blaustrumpf, aber ebenso positive, wenn etwa die Detektivin im Krimi Archivrecherchen betreibt. Auch eine Episode von 'Kommissar Rex' spielte in einer Bibliothek: Im Laufe der Folge wurde jemand in der Kompaktregalanlage der Nationalbibliothek zerquetscht."

In der Realität könne das nicht passieren, gibt Seissl Entwarnung: "Die meisten dieser Regalanlagen haben einen Stoppmechanismus, man muss sich nur dagegen lehnen."

Einmal im Jahr versucht Seissl, sich ein paar Wochen frei zu nehmen und ihr "Lieblingsreiseland" Afrika zu besuchen. In einer kleinen Gruppe hat sie im abenteuerlichen Campingurlaub bereits Botswana, Namibia und Tansania bereist: "Ich habe vorher nicht gewusst, dass mir wilde Tiere so gut gefallen würden", lächelt Seissl. Und wenn sie die Spitze des Kilimandscharo erklommen hätte, würde man ihr's auch glauben. (sk)

Quelle: „dieuniversitaet-online.at“, heute:,uni:view Magazin“ vom 14.12.2004, Autorin: Simone Kremsberger

Abb. 19: Beitrag »Maria Seissl: Organisatorin, Wissensmanagerin und Abenteurerin«. In: dieuniversitaet-online.at von Simone Kremsberger, 14.12.2004. Foto: Niklas Jelinek 
Open-Access-Publikation im Sinne der CC-Lizenz BY-NC-ND 4.0

(c) 2019, Vandenhoeck \& Ruprecht GmbH \& Co. KG, Göttingen ISBN Print: 9783847110989 - ISBN E-Lib: 9783737010986 


\section{Anhang}

Open-Access-Publikation im Sinne der CC-Lizenz BY-NC-ND 4.0

(c) 2019, Vandenhoeck \& Ruprecht GmbH \& Co. KG, Göttingen 
Open-Access-Publikation im Sinne der CC-Lizenz BY-NC-ND 4.0

(c) 2019, Vandenhoeck \& Ruprecht GmbH \& Co. KG, Göttingen ISBN Print: 9783847110989 - ISBN E-Lib: 9783737010986 


\section{Autorinnen und Autoren}

Stefan Alker-Windbichler, ORCID iD: https://orcid.org/0000-0002-8639-5845, Universität Wien, Bibliotheks- und Archivwesen, Leiter der Fachbereichsbibliothek Germanistik, Nederlandistik und Skandinavistik, stefan.alkerwindbichler@univie.ac.at

Birgit Athumani Hango, Universität Wien, Bibliotheks- und Archivwesen, Leiterin der Fachbereichsbibliothek Afrikawissenschaften und Orientalistik, birgit.athumanihango@univie.ac.at

Christine Bauer, Universität Wien, Bibliotheks- und Archivwesen, Leiterin Abteilung Bestellung und Erwerbung / Hauptbibliothek und stellvertretende Leiterin Koordinierter Bestandsaufbau sowie Leiterin Functional Experts / Team Alma Erwerbung, christine.bauer@univie.ac.at

Christian Beiler, Universität Wien, Bibliotheks- und Archivwesen, Leiter des Teams Integrierte Medienbearbeitung Fachbereichsbibliotheken, christian. beiler@univie.ac.at

Guido Blechl, ORCID iD: https://orcid.org/0000-0003-4931-7239, Universität Wien, Bibliotheks- und Archivwesen, Leiter des Open Access Office, guido. blechl@univie.ac.at

Susanne Blumesberger, ORCID iD: https://orcid.org/0000-0001-9018-623X, Universität Wien, Bibliotheks- und Archivwesen, Leiterin der Abteilung Repositorienmanagement PHAIDRA-Services, susanne.blumesberger@univie.ac.at

Andreas Brandtner, ORCID iD: https://orcid.org/0000-0003-3883-6295, Freie Universität Berlin, Universitätsbibliothek, Leitender Direktor, brandtner@ub. fu-berlin.de 
Martina Cuba, Universität Wien, Bibliotheks- und Archivwesen, Leiterin der Fachbereichsbibliothek Theater-, Film- und Medienwissenschaft, martina. cuba@univie.ac.at

Kerstin Edlinger, Universität Wien, Bibliotheks- und Archivwesen, Spezialistin für eMedien in der Abteilung Formalerschließung, kerstin.edlinger@univie.ac.at

Tanja Fabian, Universität Wien, Bibliotheks- und Archivwesen, Leiterin der Fachbereichsbibliothek Publizistik- und Kommunikationswissenschaft und Informatik, tanja.fabian@univie.ac.at

Claudia Feigl, ORCID-iD: https://orcid.org/0000-0002-0469-9555, Universität Wien, Bibliotheks- und Archivwesen, Sammlungsbeauftragte der Universität Wien, claudia.feigl@univie.ac.at

Sonja Fiala, ORCID-iD: https://orcid.org/0000-0002-5492-8934, Universität Wien, Bibliotheks- und Archivwesen, Leiterin der Fachbereichsbibliothek Philosophie und Psychologie, sonja.fiala@univie.ac.at

Christa Fried, Universität Wien, Bibliotheks- und Archivwesen, Leiterin Büro Bibliotheks- und Archivwesen, christa.fried@univie.ac.at

Alfred Friedl, ORCID iD: https://orcid.org/0000-0002-9667-6624, Universität Wien, Bibliotheks- und Archivwesen, Leiter der Fachbereichsbibliothek Theologie, alfred.friedl@univie.ac.at

Alexandra Gappmayr, Universität Wien, Bibliotheks- und Archivwesen, Leiterin der Fachbereichsbibliothek Geographie und Regionalforschung, alexandra. gappmayr@univie.ac.at

Martin Gasteiner, ORCID iD: https://orcid.org/0000-0002-4616-9261, Universität Wien, Bibliotheks- und Archivwesen, Mitarbeiter im EU Horizon 2020 Projekt NEWSEYE in der Digital Humanities Forschungsgruppe, martin.gasteiner @univie.ac.at

Juan Gorraiz, ORCID iD: https://orcid.org/0000-0002-2414-3212, Universität Wien, Bibliotheks- und Archivwesen, Leiter der Abteilung Bibliometrie und Publikationsstrategien, juan.gorraiz@univie.ac.at

Michael Greil, Universität Wien, Bibliotheks- und Archivwesen, Leiter Forschungsinformationssystem u:cris, michael.greil@univie.ac.at 
Christian Gumpenberger, ORCID iD: https://orcid.org/0000-0002-9188-8716, Universität Wien, Bibliotheks- und Archivwesen, stellvertretender Leiter der Abteilung Bibliometrie und Publikationsstrategien, christian.gumpenberger @univie.ac.at

Claudia Hausberger, Universitätsbibliothek der Veterinärmedizinischen Universität Wien, Bibliotheksleitung, claudia.hausberger@vetmeduni.ac.at

Brigitte Höglinger, Universität Wien, Bibliotheks- und Archivwesen, Direktionsassistenz, brigitte.hoeglinger@univie.ac.at

Lars Kaczmirek, ORCID iD: https://orcid.org/0000-0003-4868-2541, Universität Wien, Bibliotheks- und Archivwesen, Leiter von AUSSDA - The Austrian Social Science Data Archive, lars.kaczmirek@univie.ac.at

Christina Köstner-Pemsel, ORCID iD: https://orcid.org/0000-0003-3756-1461, Universität Wien, Bibliotheks- und Archivwesen, Leiterin der Fachbereichsbibliothek Romanistik und stellvertretende Leiterin der NS-Provenienzforschung, christina.koestner@univie.ac.at

Birgit Kopar, Universität Wien, Bibliotheks- und Archivwesen, Leiterin der Abteilung Entlehnung/Lehrbuchsammlung der Hauptbibliothek und ständiges Mitglied der AG Benutzung, birgit.kopar@univie.ac.at

Birgit Kramreither, Universität Wien, Bibliotheks- und Archivwesen, Leiterin der Fachbereichsbibliothek Kultur- und Sozialanthropologie, birgit.kramreither @univie.ac.at

Brigitte Kromp, ORCID iD: https://orcid.org/0000-0002-7793-3453, Universität Wien, Bibliotheks- und Archivwesen, Leiterin der Österreichischen Zentralbibliothek für Physik \& Fachbereichsbibliothek Chemie sowie der Clearingstelle Konsortien der Universitätsbibliothek Wien, brigitte.kromp@univie.ac.at

Karin Lach, Universität Wien, Bibliotheks- und Archivwesen, Leiterin der Fachbereichsbibliothek Anglistik und Amerikanistik, karin.lach@univie.ac.at

Beate Lang, Universität Wien, Bibliotheks- und Archivwesen, Leiterin der Fachbereichsbibliothek Soziologie und Politikwissenschaft, beate.lang@univie. ac.at 
Viktoria Lang-Steixner, Universität Wien, Bibliotheks- und Archivwesen, Mitarbeiterin im Team E-Resource Management und in der Abteilung Formalerschließung sowie Leiterin der AG Alma, viktoria.lang-steixner@univie.ac.at

Benedikt Lodes, ORCID iD: https://orcid.org/0000-0003-1205-1465, Universität Wien, Bibliotheks- und Archivwesen, Leiter der Fachbereichsbibliothek $\mathrm{Mu}-$ sikwissenschaft und Leiter des UB-Chors, benedikt.lodes@univie.ac.at

Thomas Luzer, Universität Wien, Bibliotheks- und Archivwesen, Leiter der Fachbereichsbibliothek Rechtswissenschaften, thomas.luzer@univie.ac.at

Thomas Maisel, ORCID iD: https://orcid.org/0000-0003-3849-5769, Universität Wien, Bibliotheks- und Archivwesen, Leiter des Archivs der Universität Wien, thomas.maisel@univie.ac.at

Helmut Maißer, Universität Wien, Bibliotheks- und Archivwesen, Leiter der Abteilung Fernleihe und Document Delivery, helmut.maisser@univie.ac.at

Sandra Mann, Universität Wien, Bibliotheks- und Archivwesen, Leiterin Team Infodienst und Führungen, sandra.mann@univie.ac.at

Adelheid Mayer, ORCID iD: https://orcid.org/0000-0001-7923-5256, Universität Wien, Bibliotheks- und Archivwesen, Leitung ubw:innovation und Koordination Plagiatsprüfung/Hochschulschriftenservice, adelheid.mayer@univie.ac.at

Wolfgang Mayer, ORCID iD: https://orcid.org/0000-0002-8658-2528, Universität Wien, Bibliotheks- und Archivwesen, Leiter der Abteilung E-Resource Management, Koordinator von Shared Archiving Austria und Gründungsmitglied von EPICo, wolf.mayer@univie.ac.at

Gerda McNeill, ORCID iD: https://orcid.org/0000-0001-6530-584X, Universität Wien, Bibliotheks- und Archivwesen, Leiterin der Fachbereichsbibliothek Sportwissenschaft und OpenAIRE / Europäische Projekte, gerda.mcneill@ univie.ac.at

Eva-Maria Mikschi-Marischler, Universität Wien, Bibliotheks- und Archivwesen, Leiterin der Fachbereichsbibliothek Biologie und der Fachbereichsbibliothek Botanik, eva.mikschi@univie.ac.at 
Andrea Neidhart, Universität Wien, Bibliotheks- und Archivwesen, Leiterin der Fachbereichsbibliothek Wirtschaftswissenschaften und Mathematik, andrea. neidhart@univie.ac.at

Gabriele Pauer, Universität Wien, Bibliotheks- und Archivwesen, Leiterin der Fachbereichsbibliothek Ostasienwissenschaften, gabriele.pauer@univie.ac.at

Petra Pichler, Universität Wien, Bibliotheks- und Archivwesen, stellvertretende Leiterin des Teams Integrierte Medienbearbeitung Fachbereichsbibliotheken, petra.pichler@univie.ac.at

Horst Prillinger, ORCID iD: https://orcid.org/0000-0002-5992-0982, Universität Wien, Bibliotheks- und Archivwesen, Leiter des Teams Webredaktion und Fachreferent, horst.prillinger@univie.ac.at

Andrea Ramharter-Hanel, Universität Wien, Bibliotheks- und Archivwesen, Leiterin der Fachbereichsbibliothek Alte Geschichte, andrea.ramharter-hanel @univie.ac.at

Anna Ransmayr, ORCID iD: https://orcid.org/0000-0001-9303-520X, Universität Wien, Bibliotheks- und Archivwesen, Leiterin der Fachbereichsbibliothek Byzantinistik und Neogräzistik, anna.ransmayr@univie.ac.at

Wolfgang Nikolaus Rappert, ORCID iD: https://orcid.org/0000-0002-0860-0372, Universität Wien, Bibliotheks- und Archivwesen, stv. Leiter der DLE Bibliotheksund Archivwesen, Leiter des Organisationsbereichs Dezentrale Bibliotheken, nikolaus.rappert@univie.ac.at

Sonja Reisner, Universität Wien, Bibliotheks- und Archivwesen, Leiterin der Fachbereichsbibliothek Klassische Philologie, Mittel- und Neulatein und Fachreferentin für Klassische Philologie, sonja.reisner@univie.ac.at

Barbara Retschnig, Universität Wien, Bibliotheks- und Archivwesen, Leiterin der Fachbereichsbibliothek Osteuropäische Geschichte und Slawistik, barbara. retschnig@univie.ac.at

Alina Rezniczek, Universität Wien, Bibliotheks- und Archivwesen, Team Ausund Fortbildung sowie Alte und wertvolle Bestände, EOD, alina.rezniczek@ univie.ac.at 
Manuela Rohrmoser, Universität Wien, Bibliotheks- und Archivwesen, Leiterin der Abteilung ubw:helpdesk und User Training, Fachreferentin für Biologie, Physik und Naturwissenschaften, manuela.rohrmoser@univie.ac.at

Kurt Schaefer, Universität Wien, Bibliotheks- und Archivwesen, Leiter Team Sacherschließung, Fachreferent für Pflege- und Gesundheitswissenschaften, Ernährung, Pharmazie, Chemie, kurt.schaefer@univie.ac.at

Manfred Schattleitner, Universität Wien, Bibliotheks- und Archivwesen, Leiter der Fachbereichsbibliothek Erdwissenschaften und Meteorologie sowie der Fachbereichsbibliothek Astronomie, manfred.schattleitner@univie.ac.at

Elisabeth Schmid, Universität Wien, Bibliotheks- und Archivwesen, Leiterin der Abteilung Formalerschließung, elisabeth.schmid@univie.ac.at

Kurt Schneider, ORCID iD: https://orcid.org/0000-0003-3612-4841, Universität Wien, Bibliotheks- und Archivwesen, ehemaliger Leiter der Fachbereichsbibliothek Pharmazie und Ernährungswissenschaften, kurt.schneider@univie.ac.at

Monika Schreiber, Universität Wien, Bibliotheks- und Archivwesen, Leiterin der Fachbereichsbibliothek Judaistik, Mitarbeiterin im Arbeitsbereich NS-Provenienzforschung, monika.schreiber@univie.ac.at

Ariella Sobel, Universität Wien, Bibliotheks- und Archivwesen, Leiterin der Fachbereichsbibliothek Bildungswissenschaft, Sprachwissenschaft und Vergleichende Literaturwissenschaft, ariella.sobel@univie.ac.at

Alexander Sperl, Universität Wien, Bibliotheks- und Archivwesen, Leiter Arbeitsgemeinschaft AV-Medien im Unterricht, alexander.sperl@univie.ac.at

Martin Steinreiber, Universität Wien, Bibliotheks- und Archivwesen, Leiter der Fachbereichsbibliothek Kunstgeschichte, martin.steinreiber@univie.ac.at

Pamela Stückler, ORCID iD: https://orcid.org/0000-0002-2953-2217, Universität Wien, Bibliotheks- und Archivwesen, Leitung Team Öffentlichkeitsarbeit, Alte und wertvolle Bestände der Hauptbibliothek und E-Books on Demand, pamela.stueckler@univie.ac.at

Markus Stumpf, ORCID iD: https://orcid.org/0000-0003-4946-9988, Universität Wien, Bibliotheks- und Archivwesen, Leiter der Fachbereichsbibliothek Zeitgeschichte und der NS-Provenienzforschung, markus.stumpf@univie.ac.at 
Matthias Svojtka, ORCID iD: https://orcid.org/0000-0001-7511-3964, Universität Wien, Bibliotheks- und Archivwesen, stellvertretender Leiter der Fachbereichsbibliothek Botanik, matthias.svojtka@univie.ac.at

Harald Tersch, ORCID iD: https://orcid.org/0000-0003-1800-8679, Universität Wien, Bibliotheks- und Archivwesen, Leiter der Fachbereichsbibliothek Geschichtswissenschaften, harald.tersch@univie.ac.at

Ursula Ulrych, ORCID iD: https://orcid.org/0000-0002-0549-2716, Universität Wien, Bibliotheks- und Archivwesen, Abteilung Bibliometrie und Publikationsstrategien, ursula.ulrych@univie.ac.at

Stefan Wiborny, Universität Wien, Bibliotheks- und Archivwesen, stellvertretender Leiter der Abteilung Entlehnung/Lehrbuchsammlung der Hauptbibliothek, stefan.wiborny@univie.ac.at

Susanne Wicha, Universität Wien, Bibliotheks- und Archivwesen, Leiterin der Fachbereichsbibliothek Europäische Ethnologie und Mitarbeiterin im Arbeitsbereich NS-Provenienzforschung, susanne.wicha@univie.ac.at

Martin Wieland, ORCID iD: https://orcid.org/0000-0002-4912-3779, Universität Wien, Bibliotheks- und Archivwesen, Abteilung Bibliometrie und Publikationsstrategien, martin.wieland@univie.ac.at

Ute Wödl, Kammer für Arbeiter und Angestellte für Wien, Abteilung BibliothekWissen-Information, Abteilungsleitung, ute.woedl@akwien.at

Michaela Zemanek, Universität Wien, Bibliotheks- und Archivwesen, ehemalige Leiterin der Fachbereichsbibliothek Psychologie, michaela.zemanek@univie. ac.at

Sandra Zoglauer, Universität Wien, Bibliotheks- und Archivwesen, Leiterin der Fachbereichsbibliothek Archäologien und Numismatik, sandra.zoglauer@ univie.ac.at 


\section{Reihen der Vienna University Press}

650 Jahre Universität Wien - Aufbruch ins neue Jahrhundert

Historische Kommission und Forum Zeitgeschichte der Universität Wien (Hg.)

Bibliothek im Kontext

Stefan Alker-Windbichler / Murray G. Hall / Markus Stumpf (Hg.)

Broken Narratives

Philologisch-Kulturwissenschaftliche Fakultät der Universität Wien (Hg.)

\section{Gunnar Hering Lectures}

Maria A. Stassinopoulou (Hg.)

Kommunikation im Fokus. Arbeiten zur angewandten Linguistik Rudolf de Cillia / Helmut Gruber (Hg.)

Manuscripta theatralia. Schriftenreihe zu raren Dokumenten und Archivalien im Fokus kulturhistorischer Grundlagenforschung

Andreas Brandtner / Martina Cuba / Friedemann Kreuder / Birgit Peter (Hg.)

Migrations- und Integrationsforschung. Multidisziplinäre Perspektiven

Heinz Fassmann / Richard Potz / Hildegard Weiss (Hg.)

\section{Moving Byzantium}

Claudia Rapp / Johannes Preiser-Kapeller (Hg.)

Poetik, Exegese und Narrative. Studien zur jüdischen Literatur und Kunst

Gerhard Langer / Carol Bakhos / Klaus S. Davidowicz / Constanza Cordoni (Hg.)

\section{Religion and Transformation in Contemporary European Society}

Kurt Appel / Christian Danz / Jakob Helmut Deibl / Rüdiger Lohlker / Richard Potz / Sieglinde Rosenberger (Hg.)

\section{Schriften der Wiener Germanistik}

Eva Horn / Roland Innerhofer / Matthias Meyer / Stephan Müller / Annegret Pelz /

Michael Rohrwasser / Konstanze Fliedl (Hg.)

Schriften des Archivs der Universität Wien

Thomas Maisel / Kurt Mühlberger / Johannes Seidl (Hg.)

Sigmund Freuds Werke. Wiener Interdisziplinäre Kommentare

Marlen Bidwell-Steiner / Daniela Finzi / Patrizia Giampieri-Deutsch / Christian Huber /

Friedrich Schipper / Herman Westerink (Hg.)

Theater - Film - Medien

Klemens Gruber / Stefan Hulfeld / Christian Schulte (Hg.)

Vienna Studies on Constitutionalism and Legal Culture

Stefan Hammer / René Kuppe / Christian Stadler (Hg.) 


\section{Wiener Arbeiten zur Linguistik}

Alexandra N. Lenz / Melanie Malzahn / Eva-Maria Remberger / Nikolaus Ritt (Hg.)

\section{Wiener Forum für Theologie und Religionswissenschaft}

Evangelisch-Theologische Fakultät / Katholisch-Theologische Fakultät /

Institut für Islamische Studien der Universität Wien (Hg.)

\section{Wiener Galizien-Studien}

Christoph Augustynowicz / Kerstin Susanne Jobst / Andreas Kappeler / Andrea Komlosy /

Annegret Pelz / Dieter Segert / Olaf Terpitz / Tatjana Thelen / Philipp Ther / Alois Woldan (Hg.)

\section{Wiener Jahrbuch für Theologie}

Evangelisch-Theologische Fakultät Universität Wien (Hg.)

\section{Zeitgeschichte im Kontext}

Oliver Rathkolb (Hg.)

zeitgeschichte

Oliver Rathkolb (Hg.)

\section{Vandenhoeck $\bullet$ Ruprecht Verlage}

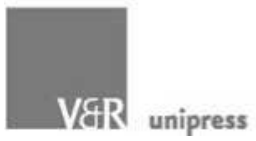

Leseproben und weitere Informationen unter www.vandenhoeck-ruprecht-verlage.com

E-Mail: info-unipress@v-r.de | Tel.: +49 (0)551 / 50 84-415 | Fax: +49 (0)551 / 50 84-333 